POROUS ANODIC METAL OXIDES

\author{
Zixue Su
}
A Thesis Submitted for the Degree of PhD at the University of St Andrews

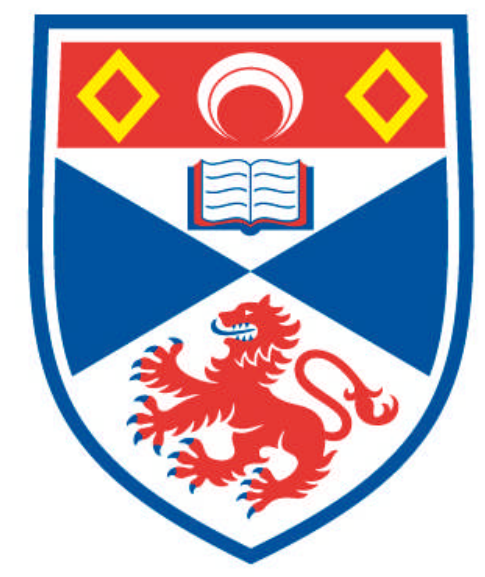

2010

Full metadata for this item is available in Research@StAndrews:FulIText at:

http://research-repository.st-andrews.ac.uk/

Please use this identifier to cite or link to this item: http://hdl.handle.net/10023/1019

This item is protected by original copyright

This item is licensed under a Creative Commons License 


\title{
Porous Anodic Metal Oxides
}

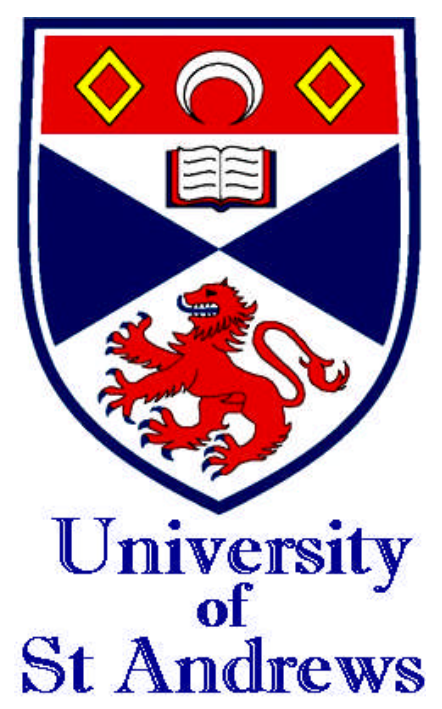

\section{Zixue Su}

\author{
PhD Dissertation \\ School of Chemistry, University of St. Andrews
}

Supervisor: Dr. Wuzong Zhou

September 2009

\section{EPSRC}

Engineering and Physical Sciences Research Council

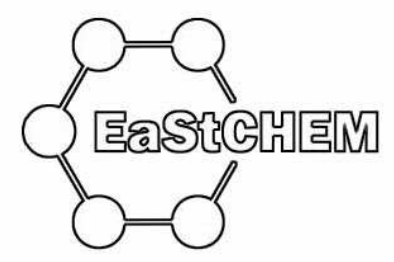




\section{Declaration}

$\mathrm{I}$,

hereby certify that this thesis, which is

approximately words in length, has been written by me, that it is the record of work carried out by me and that it has not been submitted in any previous application for a higher degree.

date signature of candidate

I was admitted as a research student in [month, year] and as a candidate for the degree of in [month, year]; the higher study for which this is a record was carried out in the University of St Andrews between [year] and .[year].

date

signature of candidate

I hereby certify that the candidate has fulfilled the conditions of the Resolution and Regulations appropriate for the degree of in the University of St Andrews and that the candidate is qualified to submit this thesis in application for that degree.

date signature of supervisor

In submitting this thesis to the University of St Andrews we understand that we are giving permission for it to be made available for use in accordance with the regulations of the University Library for the time being in force, subject to any copyright vested in the work not being affected thereby. We also understand that the title and the abstract will be published, and that a copy of the work may be made and supplied to any bona fide library or research worker, that my thesis will be electronically accessible for personal or research use unless exempt by award of an embargo as requested below, and that the library has the right to migrate my thesis into new electronic forms as required to ensure continued access to the thesis. We have obtained any third-party copyright permissions that may be required in order to allow such access and migration, or have requested the appropriate embargo below. 
Access to all of printed copy but embargo of all of electronic copy for a period

of .....2.... years (maximum five) on the following ground:

publication would be commercially damaging to the researcher, or to the supervisor, or the University;

publication would preclude future publication;

publication would be in breach of law or ethics

date

signature of supervisor 


\section{Acknowledgements}

Firstly I am cordially grateful to my supervisor Dr Wuzong Zhou for his help of imparting the knowledge of electron microscopy, crystallography, and directing me to design the experiments, solving problems in experiments and writing scientific papers and dissertation. Moreover he took care of my life in St Andrews and taught me to become a true researcher. $\mathrm{He}$ is one of the persons I respect and appreciate mostly in my life.

And I would like to thanks Prof. M. Bühl from the school of chemistry in the University of St Andrews for the theoretical computation in the field enhanced dissociation of water; Mr Shijing Lu from department of physics of Zhejiang University in China for the theoretical simulation in the ionic convection during metal anodisation.

Subsequently, .I would also like to thank Mr. Ross Blackley, who trained me on high resolution transmission electron microscopy and x-ray diffraction; Prof. Ifor Samuel of Physics Department for accessing the field emission gun SEM, Mrs Sylvia Williamson for her help of IR experiments, Miss Julie Nairn for the help of gold deposition and surface polishing.

I need to say "thank you" to Mr. Wenbo Yue who gave me many helps in discussions of my experiments and operations of HRTEM and XRD, to Xiaoxiang $\mathrm{Xu}$ who help me in the IR characterization, and to other guys in the University of St Andrews who have been so nice to me.

I should thank my parents who brought me up and always supported me. They are invaluable to me in the world.

Finally, I wish to thank EPSRC and the EaStCHEM for financial support. 


\section{Abstract}

An equifield strength model has been established to elucidate the formation mechanism for the highly ordered alumina pore arrays and titanium oxide nanotubular arrays prepared via a common electrochemical methodology, anodisation. The fundamentals of the equifield strength model was the equilibrium between the electric field driven oxidation rate of the metal and electric field enhanced dissolution rate of oxide. During the anodic oxidation of metal, pore initiation was believed to generate based on dissolution rate difference caused by inhomogeneity near the metal/oxide interface. The ionic nanoconvection driven by the electric force exerted on the space charge layer in the vicinity of electrolyte/oxide interface is established to be the main driving force of the pore ordering at the early stage of the anodisation. While the equifield strength requirement governs the following formation of the single pore and the self-ordering of random distributed pore arrays during the anodisation process.

Hexagonal patterned $\mathrm{Al}_{2} \mathrm{O}_{3}$ nanopore arrays and $\mathrm{TiO}_{2}$ nanotubular arrays have been achieved by anodisation of corresponding metal substrates in proper electrolytes. The two characteristic microstructural features of anodic aluminium oxide (AAO) and anodic titanium oxide (ATO) were investigated using scanning electron microscopy (SEM) and high resolution transmission electron microscopy (HRTEM). The observations of the hemispherical electrolyte/oxide and oxide/metal interfaces, uniform thickness of the oxide layer, as well as self-adjustment of the pore size and pore ordering can be well explained by the equifield strength model.

Field enhanced dissociation of water is extremely important in determination of the porosity of anodic metal oxide. The porosity of AAO and ATO films was found to be governed by the relative dissociation rate of water which is dependent on anodisation conditions, such as electrolyte, applied voltage, current density and electric field strength. Using an empirical method, the relations between the porosity of the AAO (ATO) films and the anodisation parameters, such as electric field strength, current density and applied voltage, have been established. Besides, the extent that an external electric field can facilitate the heterolytic dissociation of water molecule has been estimated using quantum-chemical model computations combined with the experimental aspect. With these achievements, the fabrication of anodic metal 
oxide films can be understood and controlled more precisely.

Additionally, the impacts of other factors such as the electrolyte type and the temperature effect on the morphology of the anodic products were also investigated. Some important experimental evidences on the pore diameters variation with applied voltage in the anodisation of aluminium and the titanium were obtained for future investigation of the anodic metal oxide formation processes. 


\section{Contents}

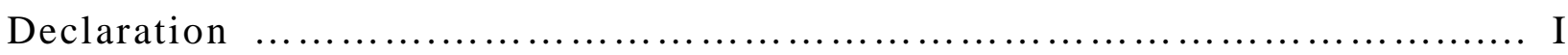

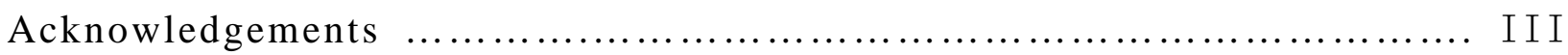

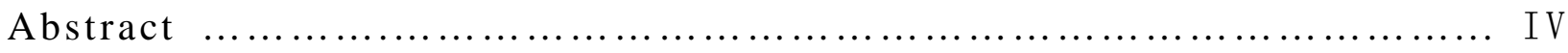

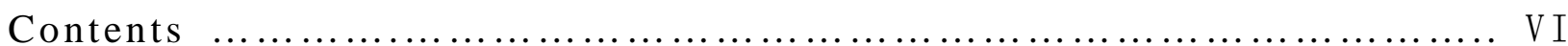

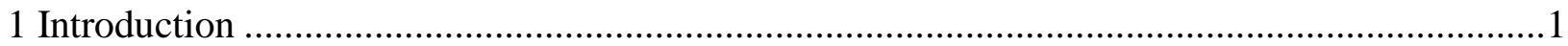

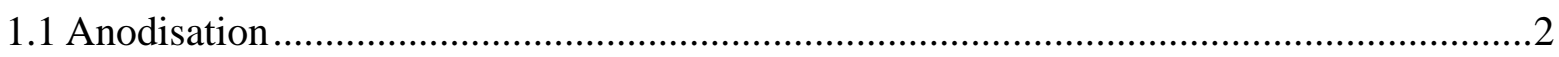

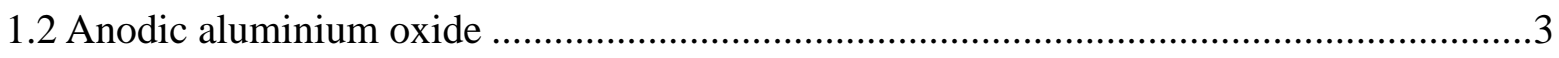

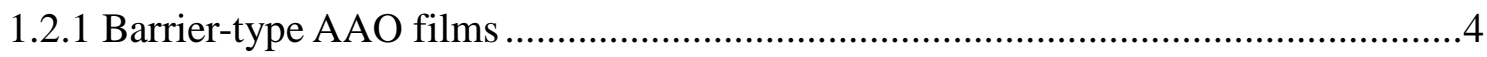

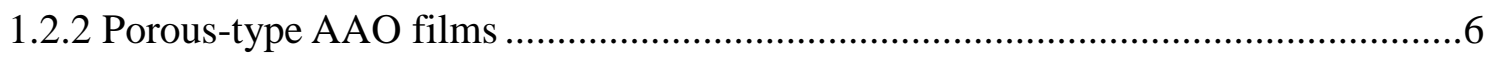

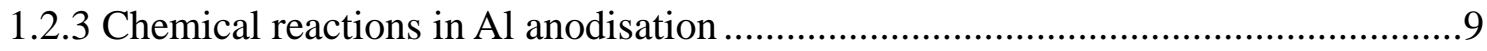

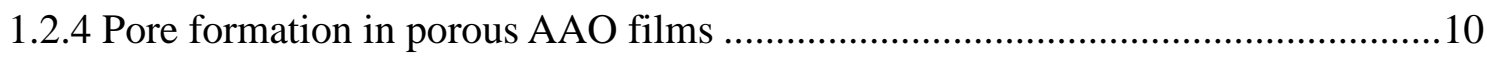

1.2.5 Self-ordering of pores in porous AAO films .................................................... 12

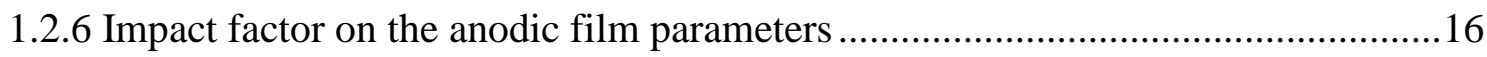

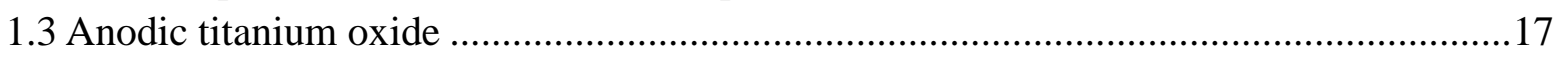

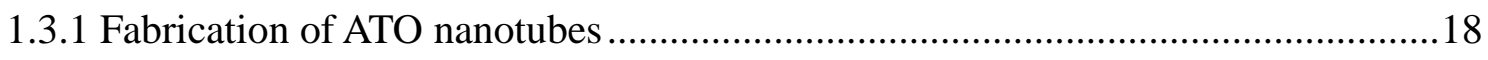

1.3.2 Formation mechanism of ATO nanotubes .........................................................22

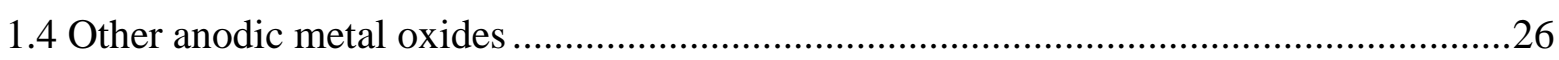

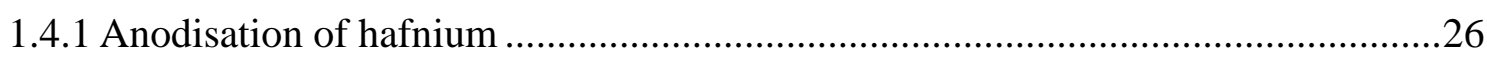

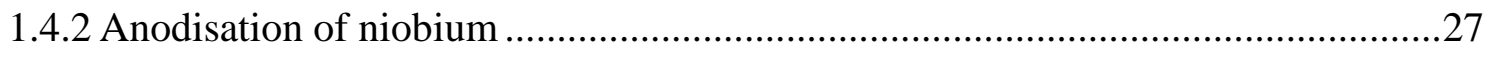

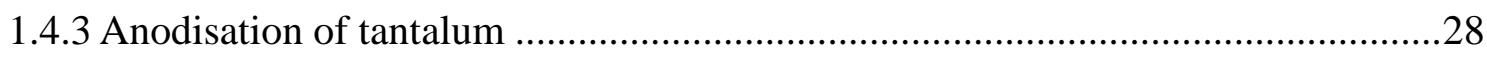

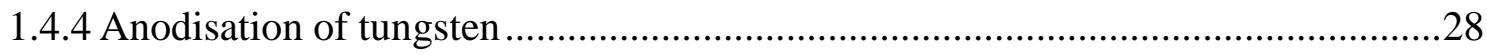

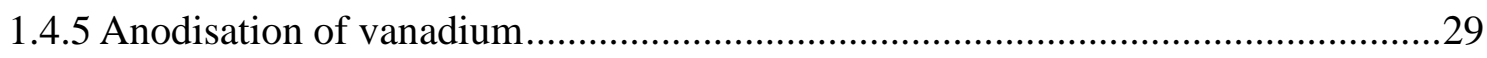

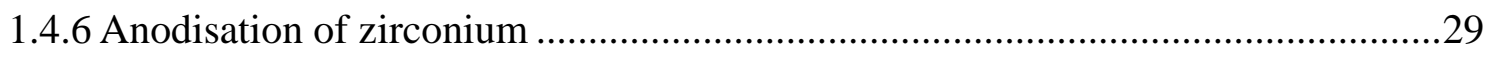

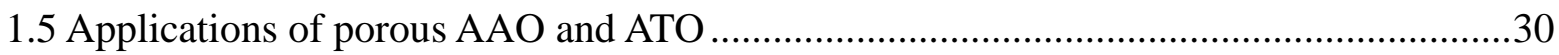

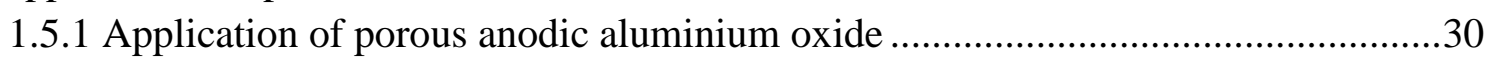

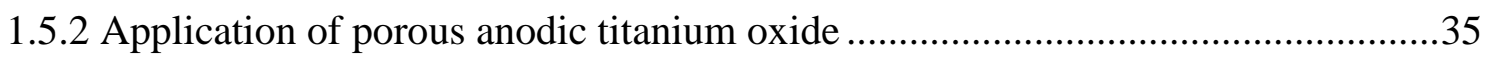

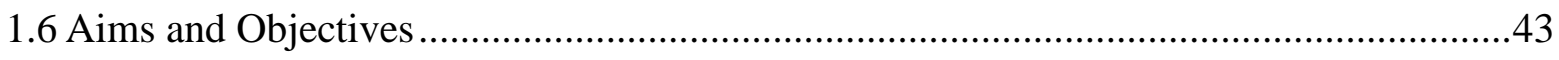

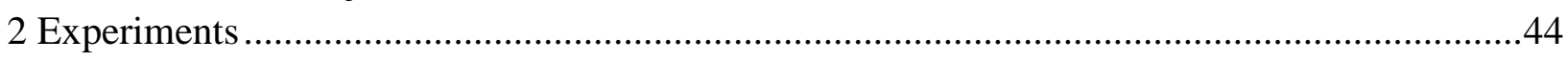

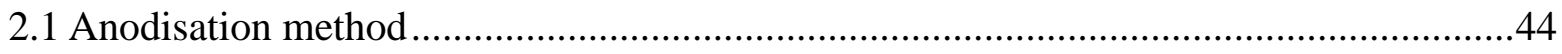

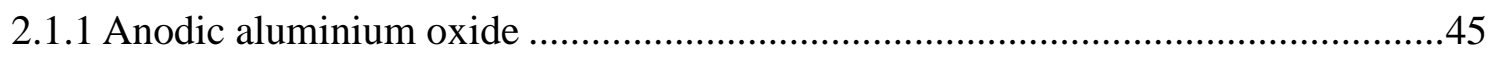

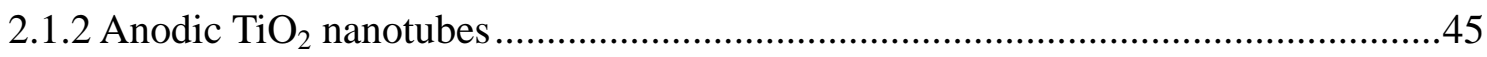

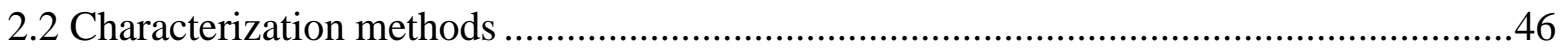

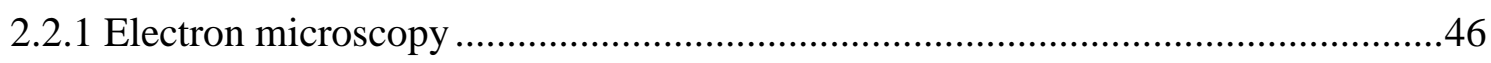

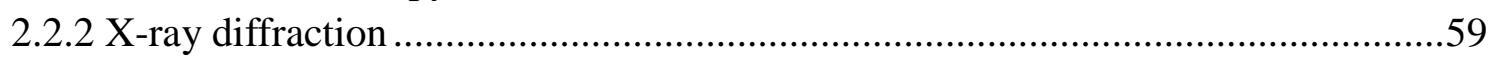

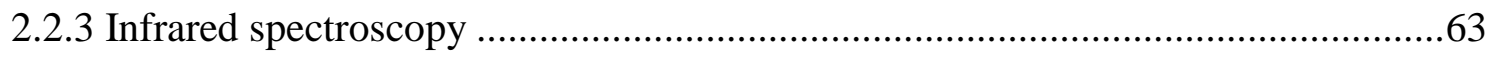

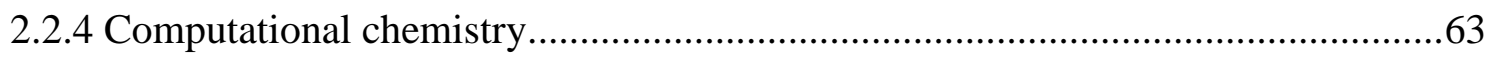

3 Formation of Self-ordered AAO Nanopore Arrays …...........................................................66

3.1 Equifield strength model and its application in the AAO formation ............................67

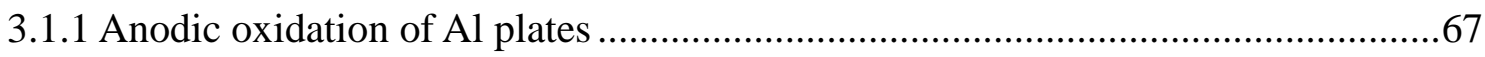




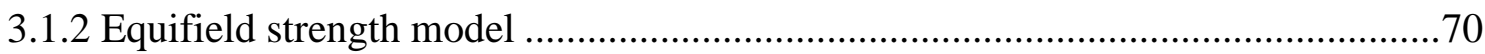

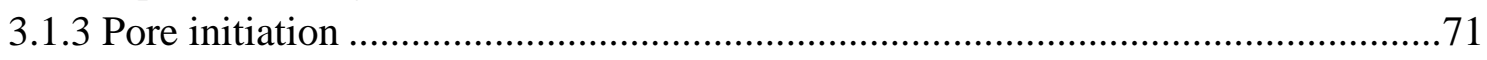

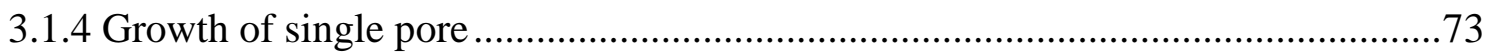

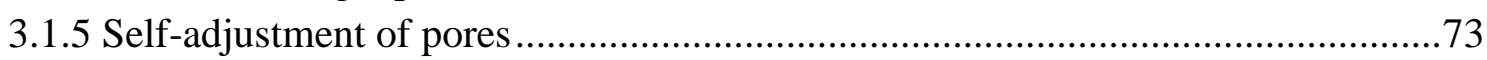

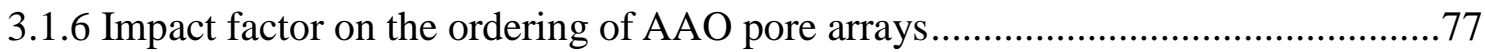

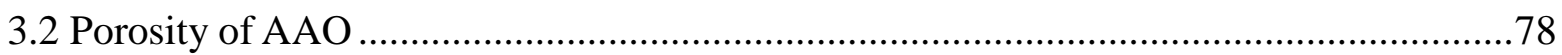

3.2.1 Relative dissociation of water and the porosity of AAO ................................... 78

3.2.2 Ratio of dissolved $\mathrm{Al}$ cations from the two interfaces .........................................82

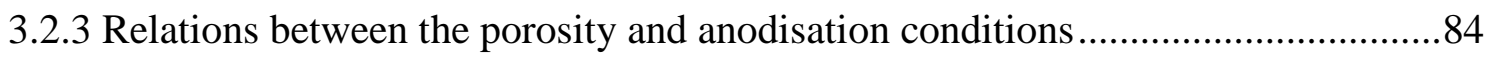

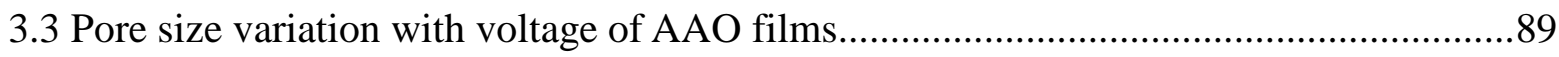

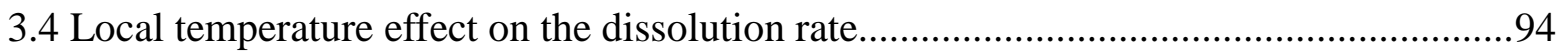

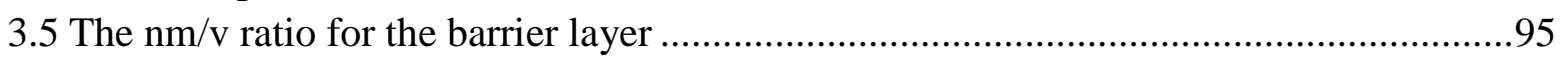

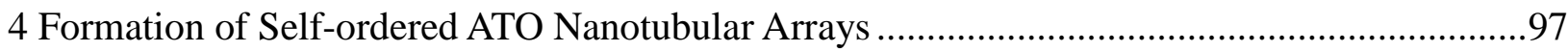

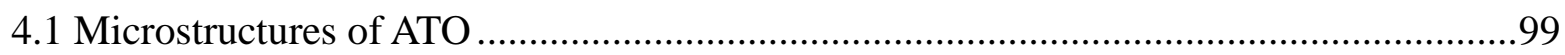

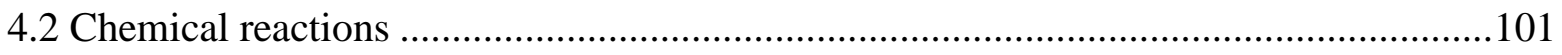

4.3 Equifield strength requirement and the ATO formation mechanism ...........................103

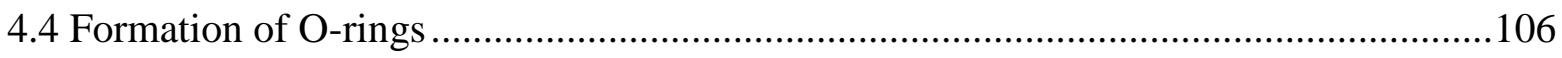

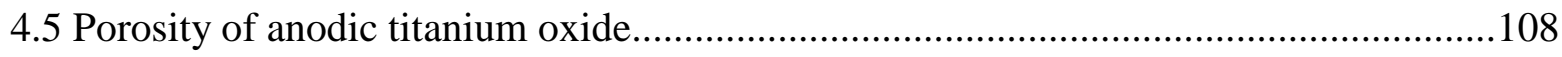

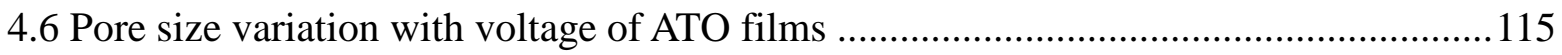

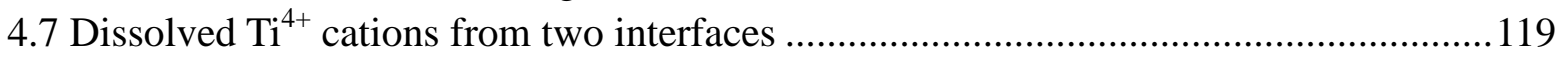

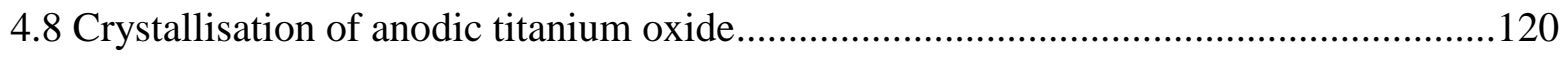

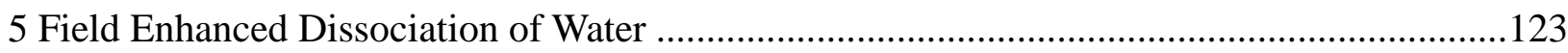

5.1 Theoretical computation for the field present water dissociation................................ 124

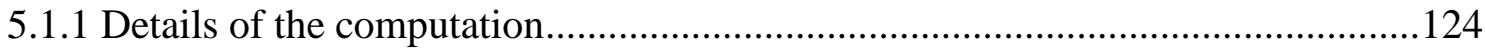

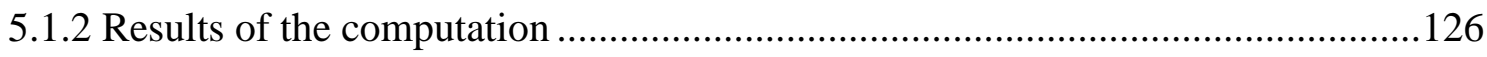

5.2 The experimental fit for the theoretical computation ............................................... 130

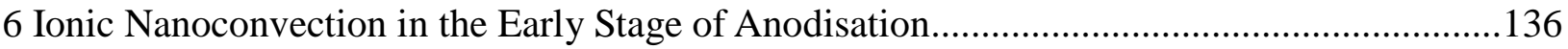

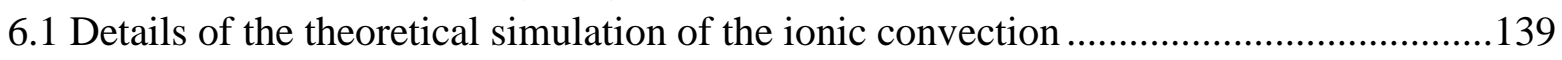

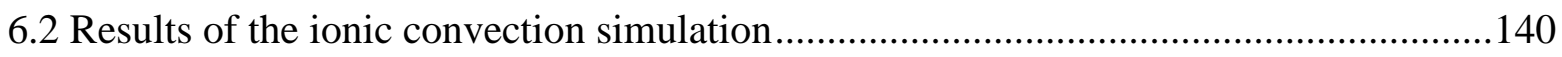

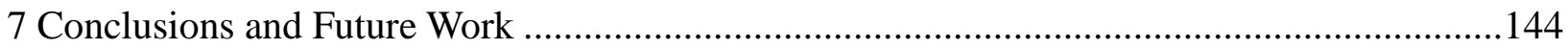

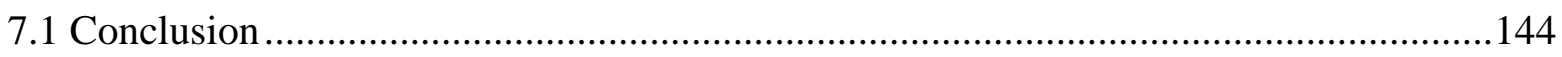

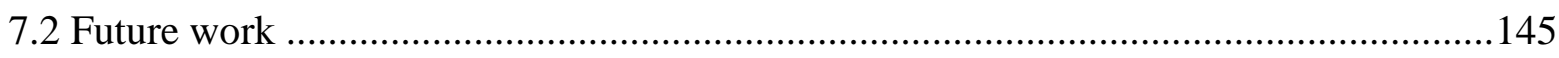

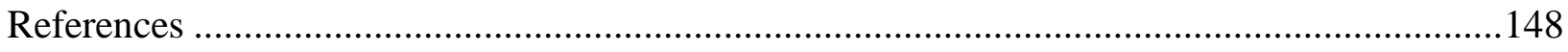

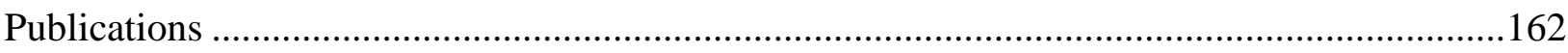




\section{Introduction}

The research realms of fabrication and application of nanomaterials have triggered tremendous technological and scientific interest from various disciplines in the recent years. Compared with the bulk materials, nanomaterials often have unique electronic and optical properties, many of which are yet to be investigated. Nanostructured metal oxide material devices have exhibited variety of high-tech applications such as dye-sensitized solar cells, ${ }^{1}$ displays and smart windows, ${ }^{2}$ and biosensors, ${ }^{3}$ lithium batteries, ${ }^{4}$ and supercapacitors. ${ }^{5}$ Indeed, to fulfill the great promise and expectation of nanomaterials, scientists and chemists have developed a number of new synthetic techniques and patterning methods, such as self-assembly, ${ }^{6}$ mechanochemistry, ${ }^{7}$ chemistry by microwave, ${ }^{8}$ lithography ${ }^{9}$ or template- and membrane-based synthesis. ${ }^{10}$ Among these methods, the anodising approach is able to build metal oxide nanopores of controllable pore size, good uniformity, array-orderly, and conformability over large areas at low cost via a simple experimental procedure.

Anodic metal oxides have diverse applications in the prevention of corrosion of metal substrates from their service environment, ${ }^{11}$ forming capacitor dielectrics, ${ }^{12,13}$ templating nanomaterials ${ }^{14-19}$ and in many other fields such as catalysis, optics and electronics. ${ }^{20-23}$ The best known porous anodic oxide, anodic aluminium oxide (AAO), was first reported 50 years ago ${ }^{24-27}$ and is now commercially available. It can be used as an ideal template for preparing various nanoparticles, nanowires and nanotubes. Porous anodic oxide films have also been achieved on surfaces of many other metals, sometimes the so-called valve metals, e.g., titanium, ${ }^{28-30}$ hafnium, ${ }^{31}$ niobium,${ }^{32}$ tantalum,${ }^{33}$ tungsten, ${ }^{34}$ vanadium, ${ }^{35}$ and zirconium. ${ }^{36}$

The concept that the formation of porous anodic metal oxides is based on two continuous processes, one being oxide dissolution at the electrolyte/oxide interface and the other being oxidation of metal at the oxide/metal interface, is widely accepted by former researchers. However, the formation mechanism of these pores, often hexagonally ordered, is much more complicated than people normally predicted. There remain a number of questions from the pore initiation at the very beginning to the formation of highly ordered patterned pore arrays at the last. Although many efforts have been made in fabrication, application and understanding the 
formation mechanism of nanoporous anodic metal oxides, ${ }^{37-42}$ there is still much work to do in order to understand fully the electrochemical process during the anodisation. Then it will be possible to optimize the anodisation conditions to manage precise control of the growth of the anodic metal oxide, finally improving and widening their scientific and industrial applications.

\subsection{Anodisation}

An oxide film can be grown on certain metals - aluminium, titanium, tantalum, etc. by an electrochemical process called anodisation. The process is called "anodisation" because the part to be treated forms the anode electrode of the processing electrical circuit. Anodisation can be used to increase the thickness of the natural oxide layer on the surface of metal parts, which can increase corrosion and wear resistance, and provides better adhesion for paint primers and glues than bare metal. Besides the conventional application in prevention of corrosion of the metal substrate from their service environment and being the capacitor dielectrics, anodic metal oxide films have widened their use in many other aspects in catalysis, optics, electronics, and in nanotechnology.

Anodic films are generally much stronger and more adherent than most types of paint and metal plating, making them less likely to crack and peel. It has been widely applied to protect aluminium alloys, but is not a useful treatment for iron or carbon steel because these metals exfoliate when oxidized; i.e. the iron oxide (also known as rust) flakes off, constantly exposing the underlying metal to corrosion. Except aluminium, porous anodic oxide films have also been achieved on surfaces of many other valve metals, such as titanium, hafnium niobium, tantalum, tungsten, vanadium, and zirconium et. al. The surfaces of these so-called valve metals could be immediately covered with a native oxide film of a few nanometers when these metals are exposed to oxygen containing surroundings. While these oxides retard the rate of reaction on the metal surface inherently, for each of these valve metals there are some process conditions that may promote growth of a thin, dense, barrier oxide of uniform thickness. The thickness and the properties of such a barrier layer vary greatly among different methods.

In an anodising cell, a piece of metal foil is used as anode by connecting it to the positive terminal of a dc power supply. The cathode is connected to the negative terminal of the supply. 
The cathode can be a plate or rod of carbon, lead, nickel, stainless steel - any electronic conductor that is unreactive (inert) in the anodising bath. When the circuit is closed, electrons are withdrawn from the metal at the positive terminal, allowing ions at the metal surface to react with water to form an oxide layer on the metal. The electrons return to the bath at the cathode where they react with hydrogen ions to make hydrogen gas.

A bath electrolyte is selected in which the oxide is insoluble, or at least dissolves at a lower rate than its deposition, and eventually an adherent oxide layer grows. The bath composition is the primary determinant of whether the film will be a barrier or porous. Barrier oxide grows in near neutral solutions in which the metal oxide is hardly soluble. Porous oxide grows in acidic electrolyte in which oxide can not only be deposited but also dissolves.

\subsection{Anodic aluminium oxide}

The history of anodisation of aluminium dates back to the beginning of the last century. Protection and decoration of $\mathrm{Al}$ and its alloys by means of an anodic treatment was developed as early as the 1920's. ${ }^{11}$ Anodisation processes have been applied for various metals such as aluminium, magnesium, niobium, tantalum, titanium, tungsten, vanadium, zinc, and zirconium, among which the anodic alumina films are most commonly applied to protect and decorate aluminium alloys. Before anodisation, aluminium metal could react with oxygen in air, forming a thin barrier oxide layer with a thickness of 2-3 nm next to the metal surface. ${ }^{43}$ This compact barrier oxide layer protects the surface from further oxidization by its service environment and is an excellent electrical insulator.

When an aluminium foil covered with a native oxide is made the anode in an typical anodising cell, the voltage across the oxide can be increased, without initiating current flow, until the field in the oxide is large enough to drive aluminium and oxygen ions through the oxide. ${ }^{44,45}$ The ionic transport across the oxide layer under a high field plays an important role in oxide formation during the anodisation of aluminium. ${ }^{46}$ Electric field strength in a range from $10^{8}$ to $10^{9} \mathrm{~V} / \mathrm{m}$ is required to drive the ions crossing the oxide layer. Once a sufficient high external voltage is applied, hydrogen ions are reduced to produce hydrogen gas at the cathode and aluminium is oxidized into $\mathrm{Al}^{3+}$ cations at the anode. The current through the oxide is an ionic current driven 
by high field, since if it is an electronic current, then water would be oxidized to evolve oxygen, which is not seen or at least not significant in experiments.

Depending on the electrolyte used, two types of anodic aluminium oxide layers could be produced: barrier-type films with uniform thickness in a near neutral electrolyte and porous anodic oxide films containing dense nanoscale pores in an acidic or alkaline electrolyte. ${ }^{47,48}$

\subsubsection{Barrier-type AAO films}

When $\mathrm{Al}$ is anodised in the electrolytes where the formed oxide film is completely insoluble such as ammonium tartrate solution, ${ }^{49}$ nonporous barrier-type films will be produced. Figure 1-1 shows a typical barrier-type AAO films grown on the top of the metal substrate.

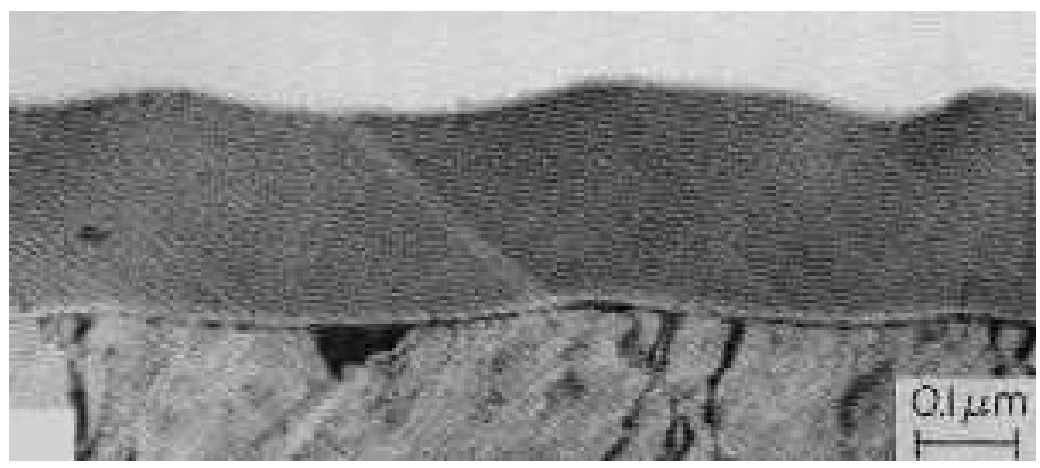

Figure 1-1. SEM image of a cross section of an amorphous aluminium oxide layer (top) grown on aluminium (bottom) in $0.16 \mathrm{M}$ ammonium tartrate at $20{ }^{\circ} \mathrm{C}$ with a current density of 10 $\mathrm{mA} / \mathrm{cm}^{2}$. The oxide thickness is about $220 \mathrm{~nm}$ at a stable voltage of $200 \mathrm{~V}$, equivalent to 1.1 nm/V. (By Furneaux et al, 1978) ${ }^{49}$

It has been generally accepted that during the formation of nonporous anodic alumina in a near neutral electrolyte, aluminium oxide simultaneously grows at both the electrolyte/oxide and oxide/metal interfaces. Oxygen-containing anions, $\mathrm{O}^{2-} / \mathrm{OH}^{-}$mainly coming from dissociation of water at the electrolyte/oxide interface move inwards to react with aluminium at the oxide/metal interface to form oxide. At the same time, $\mathrm{Al}^{3+}$ cations move outwards from the metal surface to react with water at the electrolyte/oxide interface to form oxide. As the oxide is insoluble in a 
near neutral electrolyte, a barrier layer forms at near $100 \%$ efficiency, i.e. almost all of the $\mathrm{Al}^{3+}$ cations migrating from the oxide/metal interface would contribute to the formation of $\mathrm{Al}_{2} \mathrm{O}_{3}$ at the electrolyte/oxide interface. ${ }^{50}$ Brown et al. gave a ratio of oxide formation at oxide/electrolyte and oxide/metal interfaces to be $40 \%: 60 \%$. Such films grow by counter migrations of $\mathrm{Al}^{3+}$ and $\mathrm{O}^{2-}$ ions, with the latter accounting for about $60 \%$ of the ionic current. ${ }^{51}$ Figure $1-2$ illustrates a cation transport number of 0.4 in the formation of a barrier type oxide layer at $100 \%$ efficiency by Skeldon et al. ${ }^{52}$

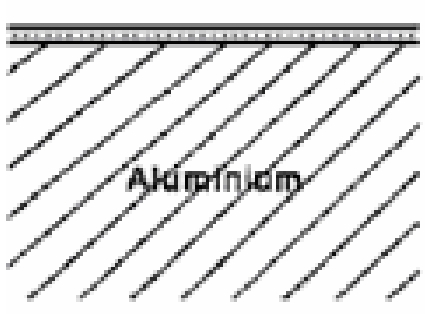

(a)

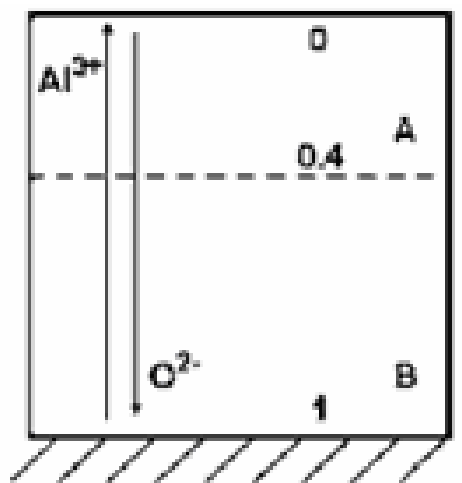

(b)

Figure 1-2. Schematic diagrams illustrating dimensional changes of an aluminium specimen following anodising. (a) Initial aluminium, with a thin air-formed oxide film. An immobile marker layer, shown as a dashed line, is incorporated into the oxide film. (b) An aluminium foil anodized at $100 \%$ efficiency, with formation of a barrier anodic film. The marker layer is located at a depth of $40 \%$ of the film thickness, in a plane corresponding to that of the original metal surface. (By Skeldon et al, 2006) ${ }^{52}$

It was reported that, when aluminium was anodised in a sodium borate-ethylene glycol electrolyte, the fraction of the total charge carried by cations $\left(\mathrm{tAl}^{3+}\right)$ was about 0.6 , whereas in aqueous ammonium citrate $\mathrm{tAl}^{3+}$ varied from 0.37 to 0.72 as the current density increased from 0.1 to $10 \mathrm{~mA} / \mathrm{cm}^{2} .{ }^{53}$ Some other values of the transport numbers of $\mathrm{Al}^{3+}$ cations were also detected. ${ }^{52,54,55}$ The total ionic current density $(j)$ under a high field conduction relates to the electric field strength (E) through an exponential law $j=j_{0} \exp (\beta \mathrm{E})$ where $j_{0}$ and $\beta$ are temperature-dependent constants involving parameters of ionic transport in a particular material. $^{44,45}$ 
The final thickness of the barrier oxide is proportional with the applied voltage, and has a small deviation with temperature and the electrolyte characteristics. A typical anodisation ratio for aluminium anodised in a near neutral electrolyte is about $1.4 \mathrm{~nm} / \mathrm{V} .^{47,56}$ Apart from the temperature of the electrolyte, barrier-type film thickness is controlled mainly by the voltage applied. The maximum film thickness attainable for barrier-type films is restricted to a voltage below the oxide breakdown voltage value, i.e., 500-700 V, which corresponds to 7000-10,000 A. ${ }^{57}$

\subsubsection{Porous-type AAO films}

When Al was anodised in acidic solution, due to the solubility of alumina in the electrolyte, instead of the nonporous uniform barrier oxide a duplex structure with a barrier layer close to the metal surface, and a porous cellular layer grown on top could be formed. Examples of this type of electrolyte are numerous, the most commonly used being sulfuric, phosphoric, chromic, and oxalic acids.

It has been known from as early as $1932^{58}$ that the porous anodic oxide film on aluminium consists of two regions: an outer region of thick porous-type oxide and a thin, compact inner region lying adjacent to the metal. From the many electronoptical investigations ${ }^{24,59-62}$ and some gas adsorption studied, ${ }^{63,64}$ the structure of the anodic alumina films formed in acidic electrolyte turned to be the close packed near hexagonal pores array with a narrow distribution of pore size. $^{24}$

During the formation of porous-type anodic aluminium oxide, the oxide film grows when oxygen containing ions $\left(\mathrm{O}^{2-} / \mathrm{OH}^{-}\right)$migrate from the electrolyte through the oxide layer at the pore bottom driven by the high field present. Simultaneously Al is oxidized at the oxide/metal interface and $\mathrm{Al}^{3+}$ cations are ejected into the solution. In the presence of field induced acidic dissolution, deep pores with a hexagonally ordered array structure can be formed. The pore diameter was found to depend on the temperature and $\mathrm{pH}$ value of the electrolyte, as well as the applied voltage. The bottom of pores consists of a barrier layer over the metallic Al surface. Both the metal/oxide and oxide/solution interfaces become somehow curved. As aluminium metal is oxidized, the metal/oxide interface moves into the metal. The pores grow as the equilibrium of 
dissolution and oxidation happens at the both side of the oxide. The cell wall and pores increase in height, that is, the film thickens. The cell and pore diameters retain a fixed value. The barrier oxide thickness remains constant as the film thickens.

A porous anodic alumina film with a rough hexagonal patterned structure was first revealed by Keller et al in 1953 using the scanning electron microscope. ${ }^{24}$ Diggle et al gave a detailed review on the progress of aluminium anodisation in the fabrication, characterization and theoretical models from the year 1930 to $1968 .^{47}$ Later, a series of new techniques such as transmission electron microscopy (TEM), microtome, marker methods and isotopic tracer were employed leading to a deep insight in the growth process of alumina oxide films. ${ }^{25,39,65,66}$ Figure 1-3 show typical images for the porous type AAO films: a thin barrier oxide is at the base of each pore and in the vicinity of the metal/oxide interface each pore is centered over a scallop-shaped depression in the metal. Pores are separated by oxide comprising the cell walls.

Perfect hexagonal anodic alumina pore arrays with a very narrow size distribution and extremely high aspect ratios over an area of micron scale were grown by Masuda and co-workers via a two step anodisation process ${ }^{40,67}$ : In the first step, Al foil was anodised for more than $10 \mathrm{~h}$, then the produced AAO film was wet etched away by the so-called P-C etch at $80^{\circ} \mathrm{C}$ using a mixture of $35 \mathrm{ml} / \mathrm{l} 85 \% \mathrm{H}_{3} \mathrm{PO}_{4}$ and $20 \mathrm{~g} / \mathrm{CrO}_{3}$. The second step was re-anodisation of the aluminium foil with a periodic surface roughness. Finally a perfect hexagonal ordered porous alumina membrane with $100 \mathrm{~nm}$ interpore distance prevailed. This discovery was a breakthrough in the preparation of 2D-polydomain porous alumina structures with a very narrow size distribution and extremely high aspect ratios. Soon after that, they successfully obtained perfect hexagonal patterned anodic alumina films with an area as large as $2 \mathrm{~mm} \times 2 \mathrm{~mm}$ by a nanoprinting method. ${ }^{68}$ A perfect hexagonally patterned SiC surface created by electron beam lithography was used to "nanoprint" the Al surface prior to anodisation, leading to a perfect hexagonal anodic alumina film. These reports confirmed that a patterned pore array can guide the pore growth. Besides, Bandyopadhyay et $a l^{69}$ found that hexagonal ordering could also be obtained via a high current density electropolishing process. Numerous other groups, not mentioned here specifically, have also contributed to an improvement of porous alumina structures. 


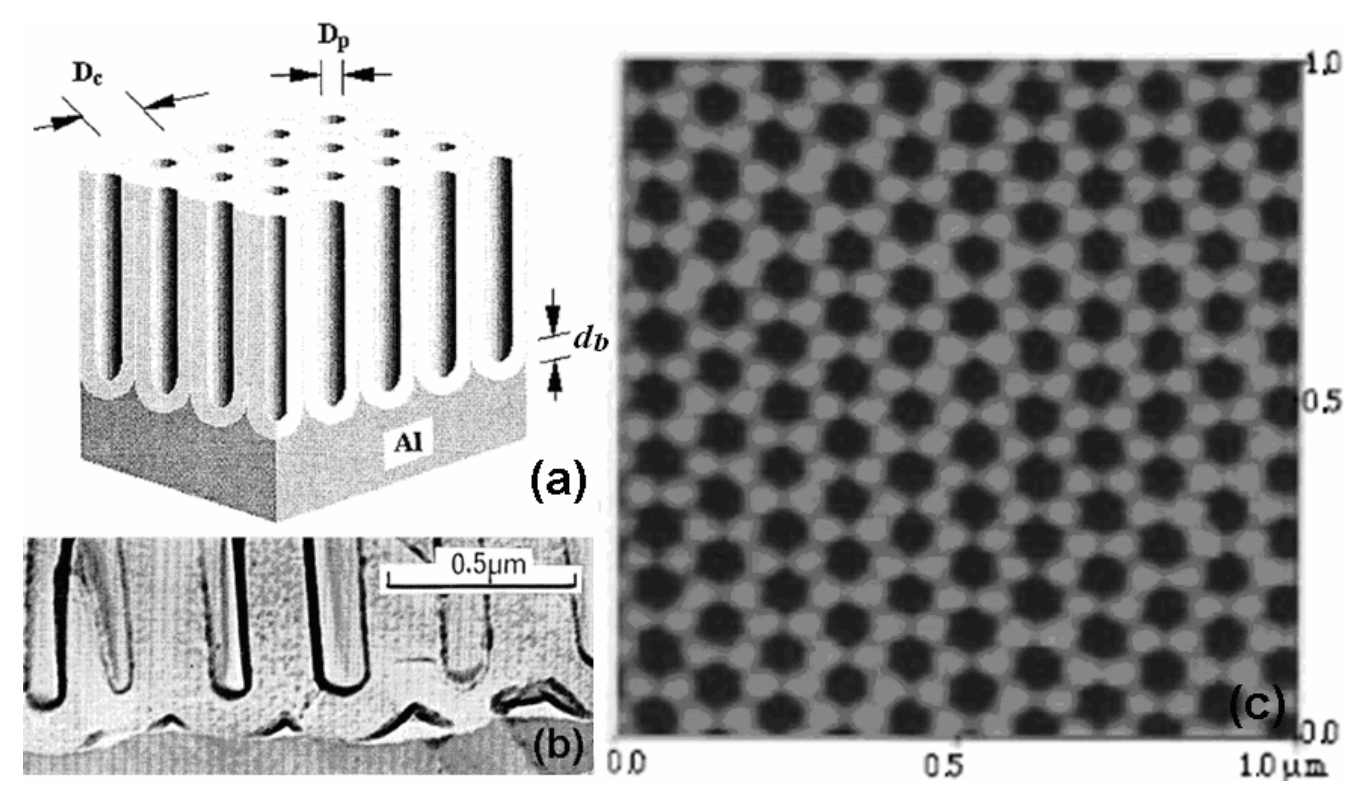

Figure 1-3. (a) An ideal hexagonal porous anodic alumina film top on an impervious "barrier" oxide deposited on the metallic $\mathrm{Al}$ bottom. The thickness of the barrier is $d_{b}$, and the pores in the hexagonal array has a diameter of $D_{p}$ with a cell (repeat) distance $D_{c}$. (By Li et al, 1998) ${ }^{48}$ (b) Section view of a film formed in phosphoric acid showing the duplex structure of the porous type anodic alumina films. (By O'Sullivan et al, 1970) ${ }^{25}$ (c) AFM image of surface of anodic alumina film grown at $40 \mathrm{~V}$ in $3 \% \mathrm{H}_{2} \mathrm{C}_{2} \mathrm{O}_{4}$ by the two-step anodisation process at $15{ }^{0} \mathrm{C}$. (By $\mathrm{Li}$ et al, 1998) $)^{48}$

Different from the barrier-type anodic films, the thickness of the porous-type anodic alumina films depends upon the current density and anodisation time. Thus a high voltage is not needed to grow a thick porous-type oxide film as the case of barrier-type oxide film. In a prolonged anodisation, porous AAO film with a length up to 100 micrometers could be achieved easily. The aspect ratio of the film thickness versus the pore diameter could commonly arrive at an order of 1000:1. ${ }^{70}$ For porous films, apart from the current density and time, the electrolyte temperature is an important criterion in determining the film thickness. At low temperatures (0-5 $\left.{ }^{\circ} \mathrm{C}\right)$ the porous film formed is thick and compact. At high temperatures $\left(60-75{ }^{\circ} \mathrm{C}\right)$ the porous film is thin and non- protective; under such conditions the process of electron polishing is achieved, i.e., where the oxide film is dissolved by the electrolyte almost as soon as it is formed. From this temperature effect on the thickness of porous films, it is clear that the electrolyte plays an important part, not only in the formation of the porous films, but also in its subsequent 
existence.

Due to its outstanding thermal, chemical, and mechanical stability, highly ordered anodic aluminium oxide has been widely used as a template in synthesis of nanomaterials in recent years. ${ }^{71-74}$ On the other hand, the driving force of self-ordering for the hexagonal patterned anodic alumina has been studied by several groups to control the film structure more precisely, fulfilling the various requirements of the vast application of anodic alumina..$^{27,75-78}$

\subsubsection{Chemical reactions in $\mathrm{Al}$ anodisation}

According to the previous researchers, the overall chemical reaction during the anodisation of aluminium can be written as: ${ }^{48}$

$2 \mathrm{Al}+3 \mathrm{H}_{2} \mathrm{O} \rightarrow \mathrm{Al}_{2} \mathrm{O}_{3}+3 \mathrm{H}_{2}$

At the metal/oxide interface, $\mathrm{Al}^{3+}$ ions form and migrate into the oxide layer:

$\mathrm{Al} \rightarrow \mathrm{Al}^{3+}+3 \mathrm{e}^{-}$

The $\mathrm{Al}^{3+}$ ions migrate outwards under the electric field across the oxide from the metal/oxide interface toward the oxide/electrolyte interface:

$2 \mathrm{Al}^{3+}+3 \mathrm{H}_{2} \mathrm{O} \rightarrow \mathrm{Al}_{2} \mathrm{O}_{3}+6 \mathrm{H}^{+}$

At the oxide/electrolyte interface the water-splitting reaction occurs ${ }^{79,80}$

$\mathrm{H}_{2} \mathrm{O} \rightarrow 2 \mathrm{H}^{+}+\mathrm{O}^{2-}$

The $\mathrm{O}^{2-}$ (oxide) ions migrate inwards under the electric field across the oxide from the oxide/electrolyte interface toward the metal/oxide interface, to form $\mathrm{Al}_{2} \mathrm{O}_{3}$ : 
$2 \mathrm{Al}+3 \mathrm{O}^{2-} \rightarrow \mathrm{Al}_{2} \mathrm{O}_{3}+6 \mathrm{e}^{-}$

In the acidic electrolyte where the oxide is soluble, the film dissolution happens:

$$
\mathrm{Al}_{2} \mathrm{O}_{3}+6 \mathrm{H}^{+} \rightarrow 2 \mathrm{Al}^{3+}+3 \mathrm{H}_{2} \mathrm{O}
$$

In addition to the above principal reactions, some anions in the electrolyte may also be incorporated in the aluminium oxide layer. The anodic alumina films consist of an inner oxide of high purity alumina and an outer oxide layer comprised of alumina which has incorporated electrolyte anions. For example, films grown in phosphoric acid electrolyte contain phosphorus species, probably as $\mathrm{PO}_{4}{ }^{3-}$ ions. ${ }^{65,66}$ However, the water of the electrolyte is still the main source of oxygen in the films. ${ }^{46}$ The inner oxide is adjacent to the oxide/metal interface, while the outer oxide is adjacent to the electrolyte/oxide interface. The distributions of such incorporated species in the anodic films are determined by their migration behaviors and the efficiency of film growth. $^{81}$

The thickness of the incorporated oxide film is strongly influenced by the ion mobility of the electrolyte species in the oxide. The boron species are immobile in the film, due to the higher energy of the $\mathrm{B}^{3+}-\mathrm{O}$ bond relative to that of the $\mathrm{Al}^{3+}-\mathrm{O}$ bond. Thus, boron species are present in the outer $40 \%$ of the thickness of films formed in ammonium pentaborate electrolyte. In contrast, sulphur and phosphorus species, probably incorporated as $\mathrm{SO}_{4}{ }^{2-}$ and $\mathrm{PO}_{4}{ }^{3-}$ ions, migrate inwards more slowly than $\mathrm{O}^{2-}$ ions so that they do not reach the metal/film interface. Phosphorus species migrate more slowly than sulphur species. Thus, in porous anodic alumina, the sulphur- and phosphorus-contaminated regions of film material extend to about 95 and $50 \%$ of the depth of the barrier region, with similar distributions in cell wall material. ${ }^{67}$

\subsubsection{Pore formation in porous AAO films}

In the recent years, much attention has been paid to porous AAO films rather than the nonporous barrier oxide layer because they have applications in nanoscience and nanotechnology. Although AAO with highly dense pores was first reported in the 1950 's ${ }^{24}$ AAO films with 
highly ordered pores were obtained 40 years later. ${ }^{40}$

It is very interesting to know how the high density pores initiate and how the hemispherically shaped pore bottom starts. The initiation and growth of pores are associated with accelerated dissolution of aluminium oxide with the influence of electric field. ${ }^{37}$ More detailed investigations $^{25,39}$ suggested that electropolishing or other pretreatment could leave a slightly scalloped surface of aluminium covered by an oxide coating layer. A scalloped "native" barrier oxide film grew over this scalloped surface; as anodisation started, pores would start at cracks and imperfections in the surface, leaving an electric field concentrated below the regions where the oxide film was thinner, thus aiding the local dissolution of oxide.

It is also suggested that during early growth, the film on ridges and protuberances becomes thicker than in the depressions. Ions move through oxide more easily at these locations because of higher film stress, impurities, or oxide flaws, and so the current concentrates at these locations. As the oxide acquires more uniform properties, the current will shift towards the thinner oxide in depressions. Because of the concave geometry that has developed, there is a slightly higher electric field in depressions, and field-assisted dissolution promotes local oxide thinning and current concentration. This initiates pores, and the pore size, density, and distribution adjust until a steady state prevails. Both these cases sound reasonable.

The problem is the cracks on the surface of the air-formed oxide layer, protuberances and the ridges seem not enough to form initially such dense pores at the entire surface, typically $10^{10} / \mathrm{cm}^{-2}{ }^{48}$. Electropolishing can create pit sizes from a few to tens of nanometers and a pit density of about $10^{10}-10^{12} / \mathrm{cm}^{-2}$, some of which can develop into pore nuclei. ${ }^{39}$ Anodisation without any pretreatment can also achieve well ordered porous alumina structure.

These initiatory pores compete with each other, only the survivors developing into the major pores. As the anodising continues, the metal/oxide interface moves into the metal, and the pores grow at the equilibrium of dissolution and oxidation taking place at the both sides of the oxide. The anodic film thickens with increasing time, while cell and pore diameters retain a constant value.

The well accepted concept for the formation of porous anodic metal oxides is based on the competition between the field-assisted oxide dissolution at the electrolyte/oxide interface and the field driven oxidation of metal at the oxide/metal interface. As illustrated in Figure 1-4, when a 
high field is present, the Al-O bond of the alumina is lengthened and weakened due to the polarization. It will then be easier for the $\mathrm{H}^{+}$ions in the electrolyte to react with and dissolve it. However, this field-dissolution process has been questioned by recent authors, ${ }^{82}$ who explained the growth of anodic pores by the high plasticity of the anodic alumina in the barrier region in the presence of ionic transport, with film growth stresses displacing material from the barrier layer towards the cell wall region during anodising.

(a)

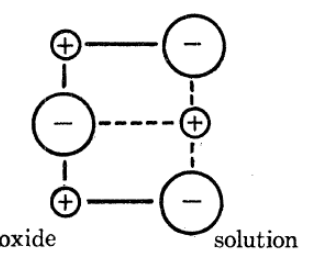

(c)

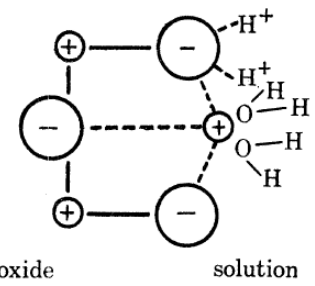

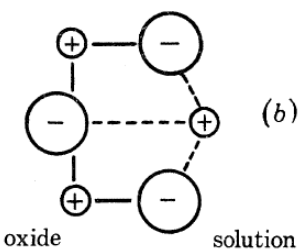

(b)

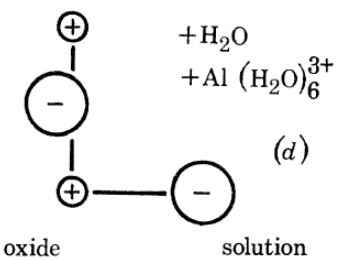

Figure 1-4. Schematic representation of the field-assisted dissolution of $\mathrm{A}_{2} \mathrm{O}_{3}$, (a) before polarization, (b) after polarization, (c) removal of $\mathrm{A}^{3+}$ and $\mathrm{O}^{2-}$ ions, and (d) the remaining oxide. (By O’Sullivan et al, 1970) ${ }^{25}$

\subsubsection{Self-ordering of pores in porous AAO films}

Since the pores generate based on the pits left by electropolishing or on the defects on the substrate surface, usually they appear as an irregular and disordered structure at the early stage of $\mathrm{Al}$ anodisation. Close packed AAO pore arrays with perfect hexagonally ordered pattern could be achieved by prolonged anodisation due to the self-organisation mechanism during films growth.

When suitable anodisation voltage and electrolyte are used, hexagonal ordered pores can be easily produced in AAO films. Perfect hexagonal anodic alumina pore arrays with a very narrow size distribution and extremely high aspect ratios over an area of a micrometer scale were grown by Masuda $e t a l^{40,83}$ via a two-step anodisation process using a $0.3 \mathrm{M}$ oxalic acid solution under a constant voltage of $40 \mathrm{~V}$ at $0{ }^{\circ} \mathrm{C}$ for over $10 \mathrm{~h}$. Masuda et al $l^{40}$ found that the ordered domains 
would grow as the anodisation prolonged. $\mathrm{Li}$ et $a l^{48}$ found in their experiments that the average ordered hexagonal domain area was a linear function of the time. ${ }^{48}$ These revealed a structure rearrangement process during the anodisation. The prolonged anodisation will tend to turn a larger area into hexagonal ordering. Later, by analysing a series of porous alumina structure prepared under different anodisation time, Nielsch et $a l^{84}$ showed that the domain size does not linearly increase with time but has a maximum (Figure 1-5). The reason the domain size decreased after a critical time was explained as an aberration from the $10 \%$ porosity rule due to a change of the $\mathrm{pH}$ value at the pore tip after lengthy anodisation. If this argument is correct, a larger electrochemical cell would maintain the properties of the electrolyte unchanged and the drop of the domain size would not be observed.

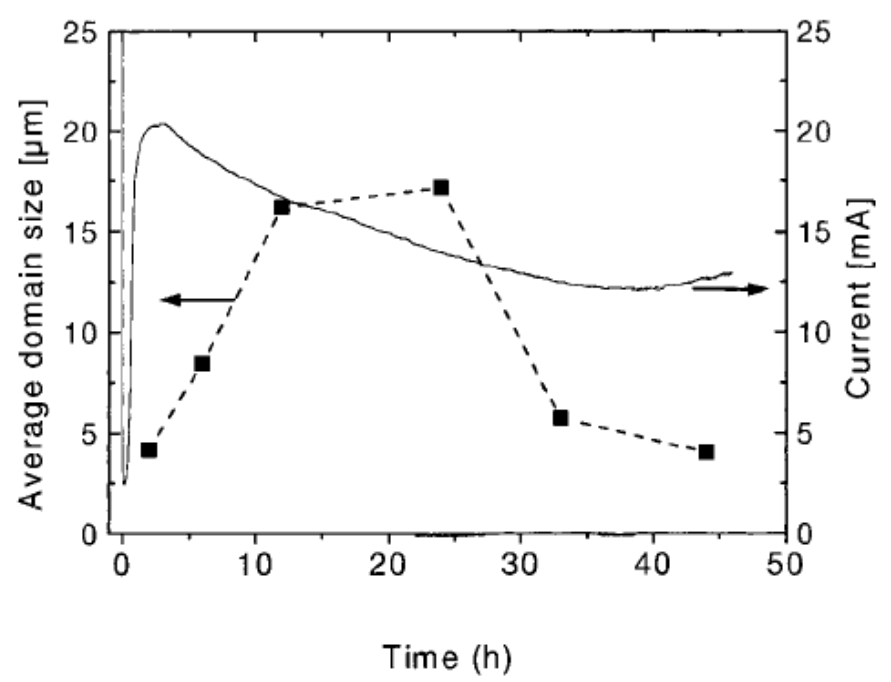

Figure 1-5. (left axis) Average domain size as a function of the anodisation time in 1 wt \% $\mathrm{H}_{3} \mathrm{PO}_{4}$ at $195 \mathrm{~V}$. The domain size is obtained by numerical picture treatment. First the domain size increases due to self-ordering, but after a critical time shown by the dashed line the domain size decreases again. (right axis) Current transient during of the same sample, showing first a current decrease typical for self-ordering, and then after a critical time the current increases. (By Nielsch et al, 2002) $)^{84}$

Singh $e t a l^{76}$ attributed the pore initiation and self organisation of the porous structures in aluminium oxide films at the initial stage to the significant elastic stress in the oxide layer. The main element of this theory is the Butler-Volmer relation ${ }^{85}$ describing an exponential 
dependence of the current on the overpotential and a dependence of the activation energies of the oxide-electrolyte interfacial reactions on the Laplace pressure and the elastic stress in the oxide layer. In the case when the effect of elastic stress is negligible, a weakly nonlinear analysis leads to formation of spatially irregular pore arrays that have been observed in experiments. In the case when the effect of elastic stress in the oxide layer is significant, the instability can transform from a long-wave type to a short-wave type. A weakly nonlinear analysis of the short-wave instability shows that it leads to the growth of spatially regular, hexagonally ordered pore arrays.
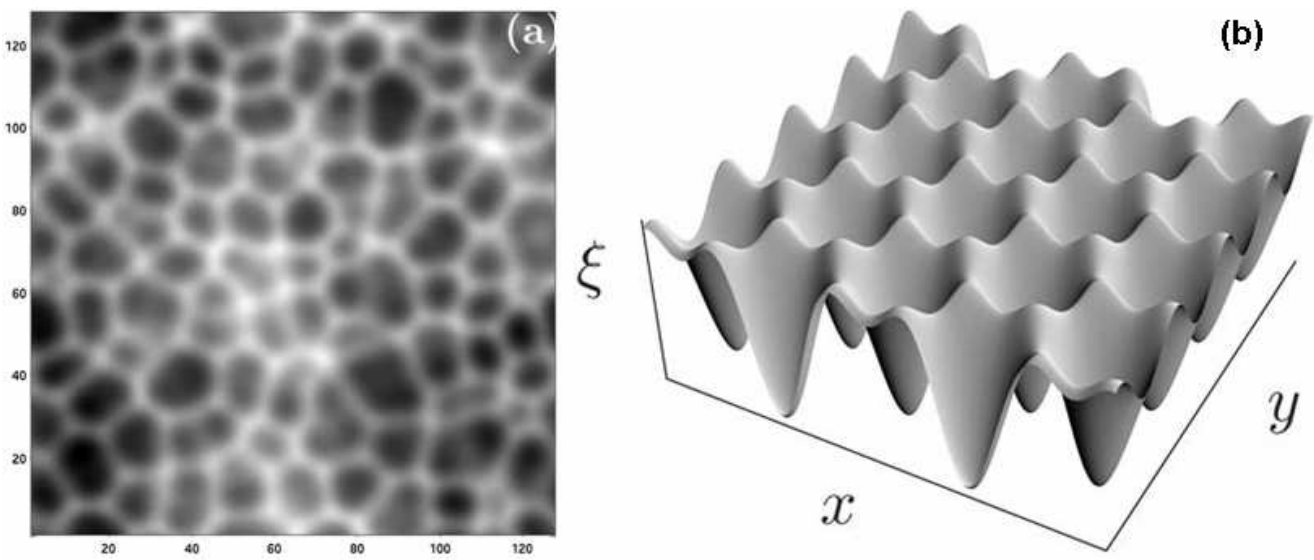

Figure 1-6. Simulation of the pore formation in AAO via a weakly nonlinear analysis. (a) When the elastic stress in the oxide layer is negligible, the simulation leads to irregular pore array. (b) When the effect of elastic stress is significant, regular pores array could be achieved. (By Singh et al, 2006) $)^{76}$

This theoretical framework studied the morphological evolution as well as the role of stress in the formation of AAO films. However, as pointed out by the authors, this model is valid only for the initial stages of instability leading to pore initiation and self organisation. It could not explain the self-adjustment process as the pores grow in depth, which is considered to be the most important factor determining the ordering of the AAO arrays.

Vorobyova et $a l^{77}$ considered distribution of an applied voltage in three main components of an electrochemical bath system: a growing oxide layer; a Helmholtz layer at the electrolyte/oxide interface; and a Gouy-Chapman space charge layer, which extends to the quasi-neutral electrolyte region. It was shown that the transformation of the Helmholtz layer is due to a 
self-organisation ion concentration distribution in the Gouy-Chapman layer, leading to a growth of highly ordered pores as shown in Figure 1-7. The cell size of the porous oxide was shown to vary as Gouy-Chapman layer thickness, which depends on the temperature, concentration, and composition of the electrolyte. It was believed that pores do not start to nucleate simultaneously across the entire surface, but do so at energetically favorable sites where most ion transfer occurs. This charge distribution model accounts for the highly ordered pore patterns at the initial anodising stage, but could not explain the self-adjustment between pores. Moreover, as observed experimentally, random distributed pores or at most poor ordering pore patterns could be observed at the beginning of the anodisation of aluminium. Only after adjustment for a sufficient long time for the initial pores, satisfactory ordering could be achieved.

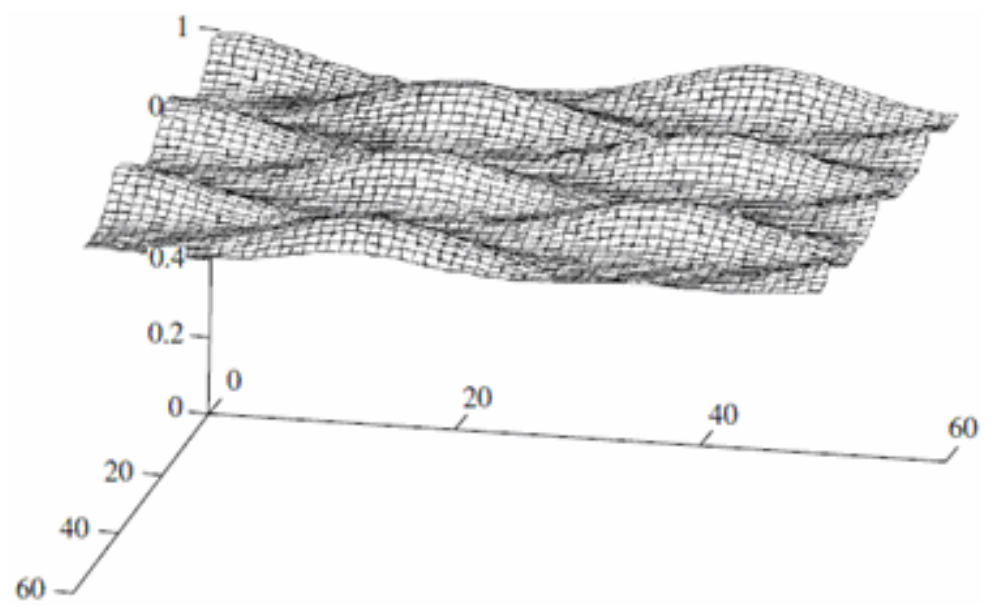

Figure 1-7. Formation of hexagonal spatial pattern of ion concentration in a Gouy-Chapman layer. The vertical axis represents normalized concentration; the horizontal axes are for coordinates (in nanometers) along the surface being anodised. (By Vorobyova et al, 2007) ${ }^{77}$

The principle of the pore ordering has been investigated extensively by a number of groups, ${ }^{25,27,40,76-78,85-87}$ while the most commonly accepted model is based on mechanical stress during the oxidation process. ${ }^{27,82}$ The ionic density of $\mathrm{Al}^{3+}$ in anodic alumina $\left(3.66 \times 10^{22} / \mathrm{cm}^{3}\right)$ corresponding to the weight density of approximately $3.1 \mathrm{~g} / \mathrm{cm}^{3}$ is significantly lower than the atomic density of aluminium metal of $6.02 \times 10^{22} / \mathrm{cm}^{3}$. The oxidation of aluminium during the anodisation may lead to significant volume expansion at an elevated anodising voltage due to higher current efficiency, as shown in Figure 1-8. The mechanical stress coming from volume 
expansion during oxide formation at the oxide/metal interface could be a possible origin of a repulsive force between neighboring pores. Jessensky et $a l^{27}$ proposed that this repulsive force could account for the self-organised formation of hexagonal pore arrays. It was also suggested that neither too large nor too small expansion would result in a long range ordering of the pores. Only a medium volume expansion of 1.2 times from aluminium to alumina, independent of the specific anodisation conditions, could lead to an ordering. ${ }^{89}$ This empirical model implies that the mechanical stress plays an important role in the self-organised process. However, it is unclear how the repulsive force, which can be either positive or negative, works in detail. As well as the detailed adjusting process, many experimental observations such as the generation of the pores, the formation of the hemispherical pore bottom, and the merging and splitting of the pores, still cannot be explained by the repulsion model.

Further to the mechanical stress model, a $10 \%$ porosity rule was proposed by Nielsch et al. ${ }^{84}$ However, by using the so-called hard-anodisation process, Lee $e t a l^{70}$ recently successfully made well ordered hexagonal pore arrays with a large aspect ratio and a porosity of $3.3 \%$, which was far below the $10 \%$ rule. This put the $10 \%$ porosity rule in question.

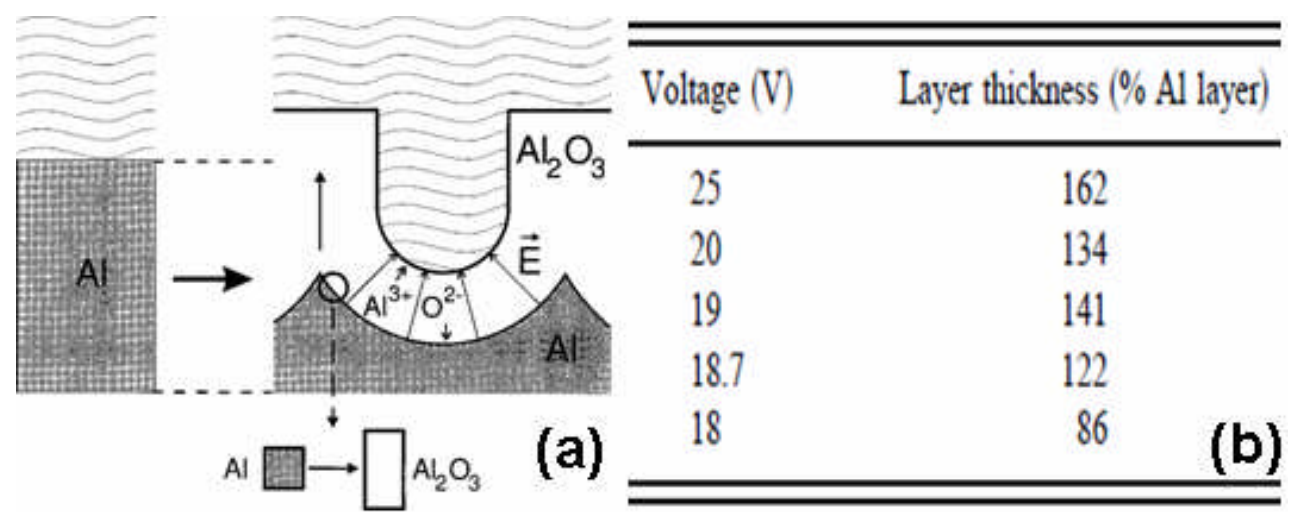

Figure 1-8. (a) Expansion of aluminium during anodic oxidation. (b) Alumina layer thickness compared to the consumed aluminium layer using 20 wt. $\% \mathrm{H}_{2} \mathrm{SO}_{4}$ at $1{ }^{\circ} \mathrm{C}$. (By Jessensky et al, $1998)^{27}$

\subsubsection{Impact factor on the anodic film parameters}

It has been observed experimentally that the cell dimension and pore diameter of AAO films 
changes significantly with the applied voltage. ${ }^{24,25,70,90}$ For example, measured from large-area hexagonal pore arrays, an interpore distance $\left(D_{\text {int }}\right)=63 \mathrm{~nm}$ when sulphuric acid $\left(\mathrm{H}_{2} \mathrm{SO}_{4}\right)$ was used as electrolyte and working voltage was $25 \mathrm{~V} ;{ }^{84,89} D_{\text {int }}=100 \mathrm{~nm}$ when oxalic acid $\left(\mathrm{H}_{2} \mathrm{C}_{2} \mathrm{O}_{4}\right)$ was used as electrolyte and voltage was $40 \mathrm{~V} ;^{40,84} D_{\text {int }}=90-140 \mathrm{~nm}$ with an aged sulphuric acid solution, a high anodisation potential up to $70 \mathrm{~V}$ and a high current density up to $200 \mathrm{~mA} / \mathrm{cm}^{2} ;^{91}$ $D_{\text {int }}=220-300 \mathrm{~nm}$ with oxalic acid at $110-150 \mathrm{~V} ;{ }^{70} D_{\text {int }}=500 \mathrm{~nm}$ when applying phosphoric acid $\left(\mathrm{H}_{3} \mathrm{PO}_{4}\right)$ and $195 \mathrm{~V} .^{84,92}$

On the other hand, the porosity of AAO films seems to be insensitive with the applied voltage. Nielsch $e a^{84}$ proposed an empirical $10 \%$ porosity rule independent of the specific anodization. However this model was in contradiction with some other reports ${ }^{70,93,94}$

O'Sullivan et $a l^{25}$ thought that the pore and cell diameters should be largely independent of the type of electrolyte, and depend mainly on the applied voltage. They gave a typical cell growth ratio of $2.5 \mathrm{~nm} / \mathrm{V}$, independent of electrolytes used. It seems that the electrolyte type and other factors such as the temperature and current density will not affect the cell voltage ratio or at most at a neglectable minor level. However, it has been reported very recently that the cell voltage ratio was found to be $2 \mathrm{~nm} / \mathrm{V}(20 \%$ smaller than $2.5 \mathrm{~nm} / \mathrm{V})$ in the hard anodisation of the aluminium by Lee et $a l^{70}$

Moreover in the research of aluminium anodised in sulfuric acid by Chu et al, ${ }^{91}$ the corresponding anodic ratio of cell diameter $(\mathrm{nm} / \mathrm{V})$ demonstrate a reverse relation to anodising potential, from $4.4 \mathrm{~nm} / \mathrm{V}$ at $8 \mathrm{~V}$ to $1.8 \mathrm{~nm} / \mathrm{V}$ at $70 \mathrm{~V}$, which is so significant that other factors rather than the voltage affecting the cell dimension must be taken into account. The authors described this phenomenon as due to the interacting repulsive force between the alumina cells which is associated with the expansion during oxide formation at the aluminium/oxide interface. This explanation is not sufficiently convincing, since the repulsive force between cells may change the dimension of a single cell, but it could not change the whole average dimension of the cells. Therefore, the cell dimension doesn't depend solely on the voltage, and the cell/voltage ratio could vary significantly with a change of the anodising voltage.

\subsection{Anodic titanium oxide}

Compared to the bulk materials, micro- or nano-structured transition metal oxides exhibit 
unique advantages in the applications such as catalysis or batteries due to the high surface area or short solid state diffusion paths. ${ }^{95,96}$ As a result, considerable research efforts worldwide focus on the design and synthesis of novel nanostructures of the transition metal oxide via chemical or electrochemical approaches. Among all transition metal oxides, $\mathrm{TiO}_{2}$ attracted the most interest by far due to its unique functional properties and wide potential application. Titania nanostructures have been successfully produced by template based, hydrothermal, and sol-gel methods. ${ }^{97-99}$ In the past decade, self-ordered titanium oxide films produced by anodisation of Ti metal have attracted intense interest from scientists and chemists all over the world.

The most significant difference between typical anodic titanium oxide (ATO) and anodic aluminium oxide (AAO) is that the latter is a continuous film with a pore array while the former consists of separated nanotubes. Several recent studies have showed that titania nanotubes have better properties compared to many other forms of titania for applications in photocatalysis, ${ }^{100,101}$ gas sensors, ${ }^{102-105}$ photoelectrolysis, ${ }^{22,106,107}$ and photovoltaics. ${ }^{23,108-110}$

As initially reported by Gong $e t a l^{28}$ in 2001 , the first generation of titania nanotubular arrays fabricated by anodisation using an aqueous HF-based electrolyte could achieve a length of about $500 \mathrm{~nm} .{ }^{28}$ The nanotube-array length was subsequently increased to about $7 \mu \mathrm{m}$ by control of the anodisation electrolyte $\mathrm{pH}$ (higher values while remaining acidic) which reduced the chemical dissolution of $\mathrm{TiO}_{2}$ during anodisation. ${ }^{29}$ Paulose $e t a l^{30}$ reported a new generation of vertically oriented $\mathrm{TiO}_{2}$ nanotubes with lengths up to $134 \mu \mathrm{m}$, by the use of various nonaqueous organic electrolytes. Meanwhile the ordering of the ATO nanotubes has also been improved dramatically. Accompanied by significant progress in the fabrication of $\mathrm{TiO}_{2}$ nanotubes via the anodisation method, scientists and chemists also made great efforts on the mechanism of the formation and self-organisation of the tube arrays.

\subsubsection{Fabrication of ATO nanotubes}

Like the anodising of aluminium, barrier-type and porous-type anodic titanium oxide films could be obtained depending on the electrolytes used. Barrier-type anodic titanium oxide films could be formed when titanium metal was anodised in sulphuric acid. Anodisation of $\mathrm{Ti}$ is widely investigated in sulfuric acid at voltages typically up to approximately $100 \mathrm{~V}$. Under these conditions, below the breakdown voltage, a uniform layer of $\mathrm{TiO}_{2}$ can be obtained. ${ }^{111-113}$ The 
exact breakdown voltage, however, depends on the exact experimental parameters (e.g., the electrochemical parameters of anodisation). For oxide films formed below the breakdown voltage, typically a growth rate of $2.5 \mathrm{~nm} / \mathrm{V}$ has been reported. ${ }^{114,115}$ The structure of the oxide films has typically been reported to be amorphous at low voltages (below $20 \mathrm{~V}^{112}$, and crystallisation takes place at higher voltages. Depending on the anodising conditions, the crystal structure has been reported to be anatase, ${ }^{114-117}$ a mixture of anatase and rutile, ${ }^{114,118}$ or rutile. ${ }^{114}$

For producing ATO nanotubes, several different electrolytes have been used so far. Therein, the most investigated electrolytes are fluoride solutions. Gong et $a l^{28}$ reported their ATO preparation in a $0.5 \mathrm{wt} \% \mathrm{HF}$ aqueous solution at room temperature using different anodising voltages, from 3 to $20 \mathrm{~V}$. Figure 1-9 shows SEM images of a typical ATO sample. Appearance of separated nanotubes became obvious at an anodising voltage of $20 \mathrm{~V}$.

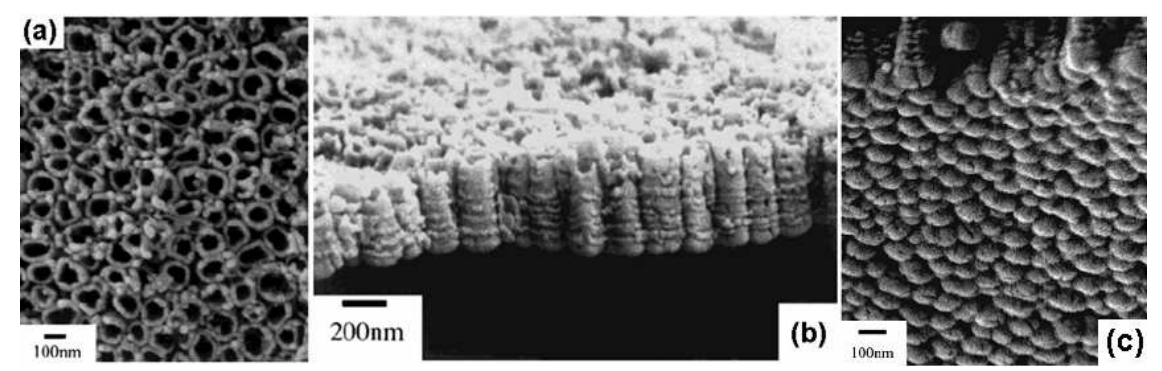

Figure 1-9. FE-SEM top view (a), cross sectional (b), and bottom view images of titanium oxide nanotubes anodised in $0.5 \mathrm{wt} \% \mathrm{HF}$ solution at $20 \mathrm{~V}$ for $20 \mathrm{~min}$. (By Gong et al, 2001) ${ }^{28}$

It was noticed that the film thickness could not be increased further from 400-500 nm using a HF-based electrolyte. Fluoride solution can help to dissolve $\mathrm{TiO}_{2}$ by forming $\left[\mathrm{TiF}_{6}\right]^{2-}$ anions. However, too strong acidity of the HF-solution results in an over fast dissolution rate of the formed $\mathrm{TiO}_{2}$ nanotubes. Mixture with other acids did not help very much, but the quality of the nanotubular arrays could be varied. Mor et $a l^{103,119}$ reported that addition of acetic acid to a 0.5 wt\% $\mathrm{HF}$ electrolyte in a 1:7 ratio resulted in more mechanically robust nanotubes without changing their shape and size. Ruan et $a l^{120}$ found that the surface morphology of nanotubular arrays anodised in an electrolyte containing $2.5 \% \mathrm{HNO}_{3}$ and $1 \% \mathrm{HF}$ at $20 \mathrm{~V}$ for $4 \mathrm{~h}$ showed a uniform, clean, regular nanotube structure with a length about $400 \mathrm{~nm}$, while an electrolyte of $0.5 \mathrm{M} \mathrm{H}_{3} \mathrm{BO}_{3}-2.5 \% \mathrm{HNO}_{3}-1 \% \mathrm{HF}$ in anodisation at $20 \mathrm{~V}$ for $4 \mathrm{~h}$ led to a greater degree of pore 
irregularity, with a nanotube length about $560 \mathrm{~nm}$. Beranek et al ${ }^{121}$ anodised titanium in $\mathrm{H}_{2} \mathrm{SO}_{4}$ electrolytes containing low concentrations of HF (0.05-0.4wt \%). It was found that under optimized electrolyte conditions, highly ordered porous $\mathrm{TiO}_{2}$ that consists of pore arrays with a single pore diameter of $140 \mathrm{~nm}$ and a pore spacing of $150 \mathrm{~nm}$ with a limiting thickness of 500 nm could be obtained.

When using a $\mathrm{KF}$ or $\mathrm{NaF}$ solution as an electrolyte, the thickness of ATO films can be significantly increased. ${ }^{122}$ The acidity of the electrolyte might be tuned by adding $\mathrm{HF}, \mathrm{H}_{2} \mathrm{SO}_{4}$ or $\mathrm{Na}_{2} \mathrm{SO}_{4}$ in order to adjust the balance of dissociation of titania at the electrolyte/oxide interface and oxidation of titanium at the oxide/metal interface. ${ }^{121,123}$ The better electrolyte is probably a $\mathrm{NH}_{4} \mathrm{~F}$-based solution. From a mixed solution of $\mathrm{NH}_{4} \mathrm{SO}_{4}$ and $\mathrm{NH}_{4} \mathrm{~F}$, the ATO film can grow up to several micrometers in thickness. ${ }^{29}$

Considering diffusion as the main effect on local acidification, ${ }^{29}$ Macak et $a l^{42}$ demonstrated an ATO sample prepared in a glycerol electrolyte with $0.5 \mathrm{wt} \% \mathrm{NH}_{4} \mathrm{~F}$ with a length of $7 \mu \mathrm{m}$ and a high degree of regularity and homogeneity as shown in Figure 1-10.
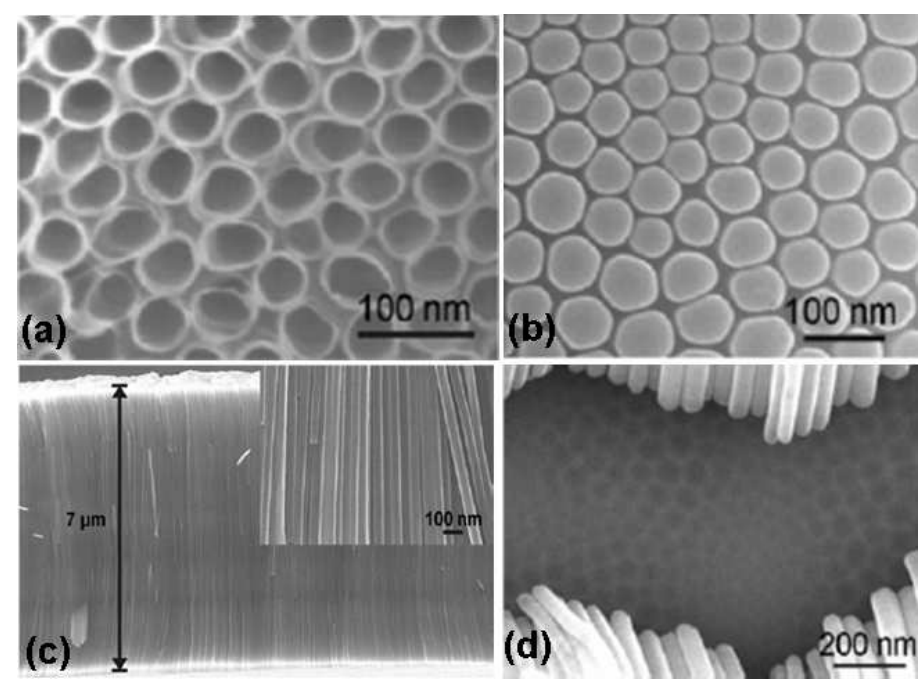

Figure 1-10. SEM images of smooth ATO with a length of $7 \mu \mathrm{m}$ produced in a glycerol electrolyte with $0.5 \mathrm{wt} \% \mathrm{NH}_{4} \mathrm{~F}$ : (a) top view; (b) bottom view; (c) cross sectional view. The inset of (c) shows the walls of the nanotubes in more details. (d) Top view of an anodised sample after removal of some nanotubes. (By Macak et al, 2005) ${ }^{42}$

In combination with either $\mathrm{HF}, \mathrm{KF}$, or $\mathrm{NaF}$ to provide fluoride ions, Grimes and 
co-workers $^{30,124}$ obtained nanotubular arrays up to approximately $220 \mu \mathrm{m}$ in length using a variety of organic electrolytes including dimethyl sulfoxide (DMSO), formamide (FA), ethylene glycol, and $N$-methylformamide (NMF) (Figure 1-11). It was suggested that, the key to successfully achieving very long nanotubular arrays was to minimize water content in the anodisation bath to less than 5\%. With organic electrolytes, donation of oxygen is more difficult in comparison with water, thus reducing the tendency to form oxide ${ }^{125}$ and slowing down the process of the nanotube growth. At the same time, the reduction in the water content reduces the chemical dissolution of the oxide in the fluorine containing electrolytes and hence aids the longer-nanotube formation.
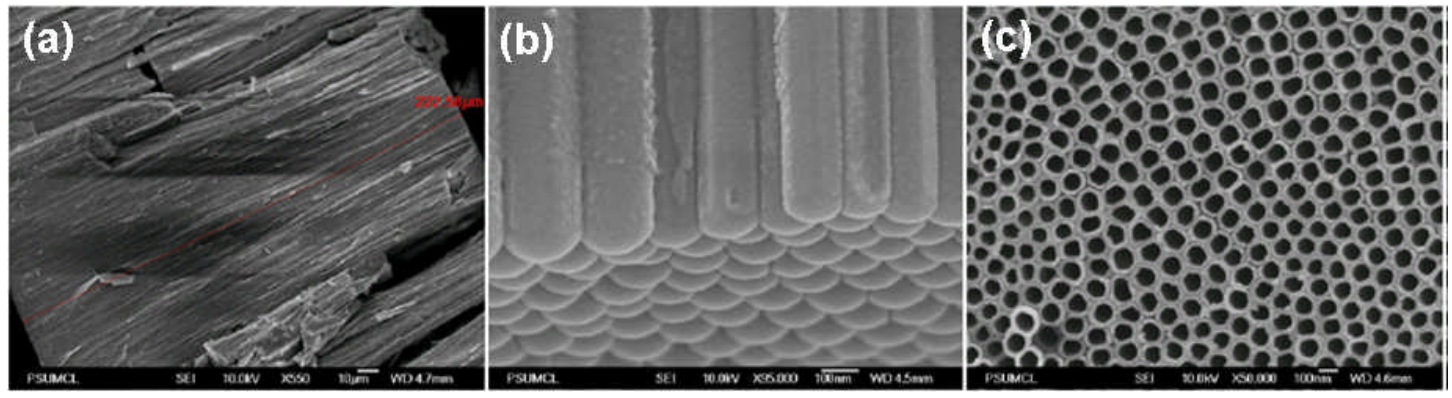

Figure 1-11. FESEM cross-sectional (a), bottom (b), and top (c) images of an ATO grown at 60 $\mathrm{V}$ in an ethylene glycol electrolyte containing $0.25 \mathrm{wt} \% \mathrm{NH}_{4} \mathrm{~F}$. (By Shankar et al, 2007) ${ }^{124}$

In fact, ATO nanotubes could also be obtained by anodising titanium metal in fluoride free electrolytes. In 2005, Nakayama et al ${ }^{126}$ reported that titania nanotubes can be obtained by anodisation in a perchloric acid solution. Hypothesizing that chlorine ions could perhaps play a role similar to that of fluorine ions during the anodisation of titanium, Richter et al ${ }^{127}$ synthesized high-aspect-ratio titania nanotubes by the anodisation of titanium in chlorine and oxalic acid containing electrolytes. Chlorine-based anodisation has an advantage over the techniques mentioned above in that it can grow long titania nanotubes (up to $60 \mu \mathrm{m}$ ) in a very short time (growth rate is $>1000$ times faster than in fluorine media; e.g., $10 \mathrm{~min}$ in chlorine-based electrolytes compared to $17 \mathrm{~h}$ in fluorine-based electrolytes for 60 - and $7-\mu \mathrm{m}$ nanotubes, respectively). Later, Richter $e{ } a l^{128}$ fabricated the ATO films in formic acid-, oxalic acid-, gluconic acid-, trichloroacetic acid-, sulfuric acid-, and hydrochloric acid-based 
electrolytes, suggesting that the chemistry for nanotube formation is versatile with regard to the acid used (Figure 1-12).

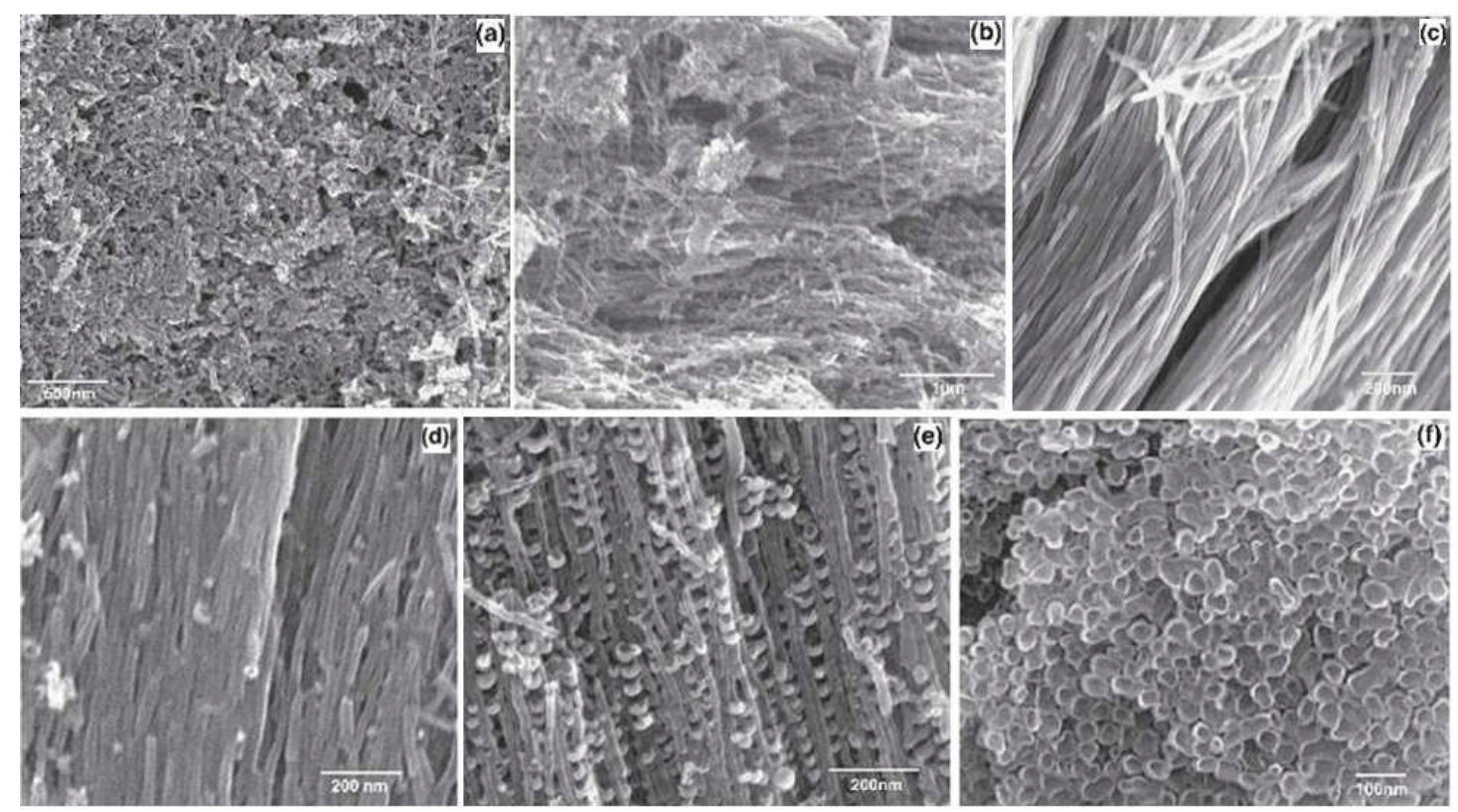

Figure 1-12. SEM images showing various types and degrees of ordering of the titanium oxide nanotubes. The specific experimental conditions are not necessarily related to the morphology depicted. (a) Totally disordered agglomerations of relatively short $(<500 \mathrm{~nm})$ nanotubes $(0.5 \mathrm{M}$ formic acid, $15.5 \mathrm{~V}$ ). (b) Highly disordered agglomerations of long nanotubes (0.5 M gluconic acid, $13 \mathrm{~V}$ ). (c) Loosely packed nanotubes with one preferred direction, allowing the formation of bundles (0.5 M gluconic acid, $12 \mathrm{~V})$. (d) Tightly packed nanotubes with one preferred direction, allowing the formation of grains (0.5 M trichloroacetic acid, $14 \mathrm{~V})$. (e) Tightly packed nanotubes with two perpendicular preferred directions, allowing the formation of interwoven bundles ( $0.5 \mathrm{M}$ formic acid, $15.5 \mathrm{~V})$. (f) Top view of a bundle clearly showing the tubular aspect of the constituents (0.02 M hydrochloric acid, $18 \mathrm{~V})$. (By Richter et al, 2007) $)^{128}$

\subsubsection{Formation mechanism of ATO nanotubes}

According to Grimes' group, ${ }^{129}$ the key processes responsible for anodic formation of titania should be the same as anodic alumina: (1) Oxide growth at the surface of the metal occurs due to interaction of the metal with $\mathrm{O}^{2-}$ or $\mathrm{OH}^{-}$ions. After the formation of an initial oxide layer, these 
anions migrate through the oxide layer reaching the metal/oxide interface where they react with the metal. (2) Metal ion $\left(\mathrm{Ti}^{4+}\right)$ migration from the metal at the metal/oxide interface; $\mathrm{Ti}^{4+}$ cations will be ejected from the metal/oxide interface under application of an electric field. (3) Field assisted dissolution of the oxide at the oxide/electrolyte interface. Due to the applied electric field the Ti-O bond undergoes polarization and is weakened, promoting dissolution of the metal cations.

$\mathrm{Ti}^{4+}$ cations dissolve into the electrolyte, and the free $\mathrm{O}^{2-}$ anions migrate towards the metal/oxide interface, to interact with the metal. The overall reactions for anodic oxidation of titanium can be represented as

$$
\begin{aligned}
& \mathrm{H}_{2} \mathrm{O} \rightarrow \mathrm{O}^{2-}+2 \mathrm{H}^{+} \\
& \mathrm{Ti}+2 \mathrm{O}^{2-} \rightarrow \mathrm{TiO}_{2}+4 \mathrm{e}^{-}
\end{aligned}
$$

In the initial stages of the anodisation process field-assisted dissolution dominates chemical dissolution due to the relatively large electric field across the thin oxide layer. Small pits formed due to the localized dissolution of the oxide, represented by the following reaction, act as pore forming centers: ${ }^{22}$

$$
\mathrm{TiO}_{2}+6 \mathrm{~F}^{-}+4 \mathrm{H}^{+} \rightarrow\left[\mathrm{TiF}_{6}\right]^{2-}+2 \mathrm{H}_{2} \mathrm{O}
$$

Then, these pits convert into bigger pores and the pore density increases. After that, the pores spread uniformly over the surface. The pore growth occurs due to the inward movement of the oxide layer at the pore bottom (barrier layer) due to processes (1.7)-(1.9). ${ }^{129}$

As the pores become deeper, the electric field in the protruded metallic region between pores increases, enhancing the field-assisted oxide growth and oxide dissolution. Subsequently inter-pore voids start forming as illustrated in Figure 1-13d. Thereafter, both voids and tubes grow in equilibrium. Close examination of FE-SEM images show the formation of small pits in the inter-pore regions which eventually leads to separation of pores and tube formation. ${ }^{129}$ 

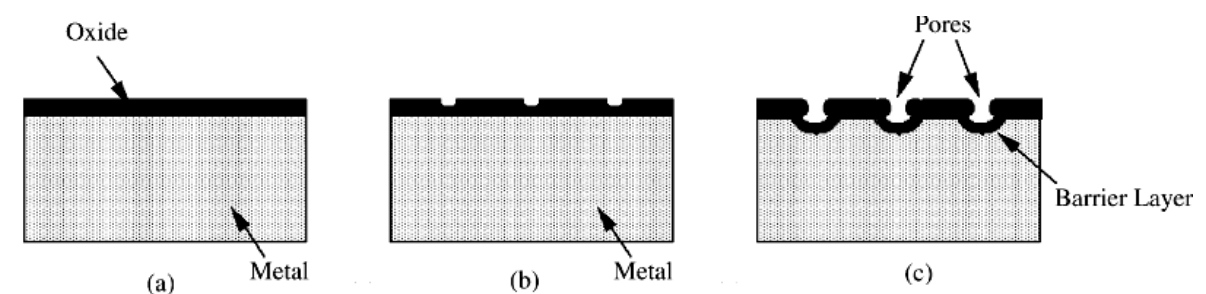

(b)

(c)
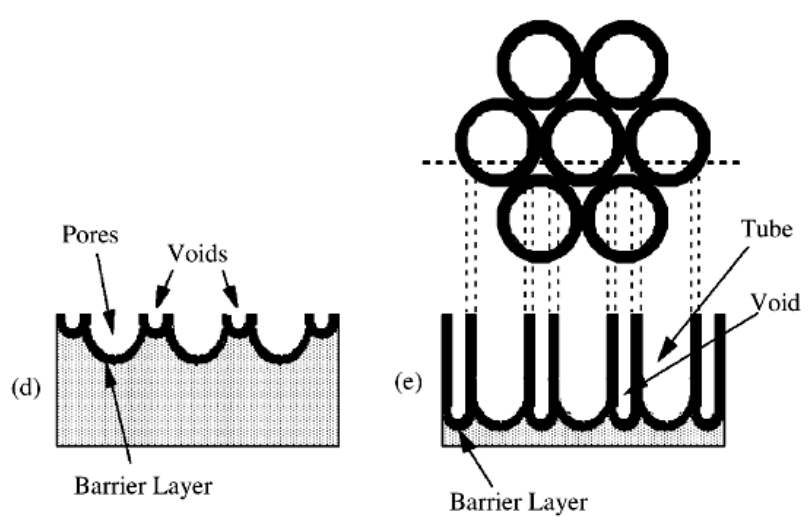

Figure 1-13. Schematic diagram of the evolution of a nanotubular array at constant anodisation voltage: (a) oxide layer formation, (b) pit formation on the oxide layer, (c) growth of the pit into scallop shaped pores, (d) metallic part between the pores undergoes oxidation and field assisted dissolution, and (e) fully developed nanotubular array with a corresponding top view. (By Mor et $a l, 2006)^{129}$

The above growth mechanism proposed by the Grime's group attributed the formation of ATO nanopores array to the electric field driven oxidation of titanium metal base and electric field enhanced dissolution of titanium oxide like the AAO case. The separation of ATO pores was attributed to the inter-pore voids which form at the enhancing the field-assisted oxide growth and oxide dissolution in the protruded metallic regions between pores. This mechanism could well explain the formation and growth of ATO pores though the self-organisation phenomenon of the ordered pores array was not elucidated. The voids growth mechanism seems imperfect in elucidating the separation of ATO pores. Due to the field enhanced oxidation and dissolution, there will be small new pores generated at the bottom of these voids. And secondary new voids will form in the joint areas between the large pores and the new pores. Therefore, this mechanism should be modified in some aspects.

However, Macak et $a l^{29,42}$ believed the mechanism of pore formation in titanium anodisation is significantly different from the relatively well investigated anodic aluminium oxide. They suggested that oxide dissolution in ATO growth was a dominant factor rather than 
the .electric-field-aided ion transport. As the dissolution rate of titanium oxide greatly depends on the local acidification in fluoride containing electrolyte, the anodic pores grow in the higher acidification at the pore bottom than the pore mouth. ${ }^{29}$

This model has been improved by the same group ${ }^{130}$ and was described as follows (Figure 1-14): in the very early stages a compact titanium oxide (and hydroxide) layer grows, then dissolution and breakdown of the barrier oxide film occurs on random sites of the surface, which act as seeds of the growth of a disordered worm-like structure underneath the remaining compact layer. A pH gradient is developed between the tips and the bottoms of pores where the lower $\mathrm{pH}$ at bottom leads to an accelerated $\mathrm{TiO}_{2}$ dissolution and pore penetration into the Ti substrate. ${ }^{29}$ Only if the current in a pore is sufficiently high it will "survive"; thus under a critical value the pore growth is "shut down", which naturally facilitates the transition from irregular to regular pores.

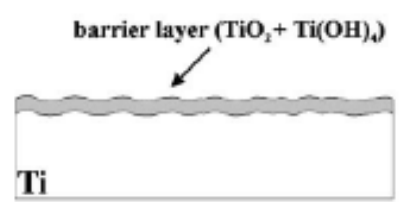

(a)

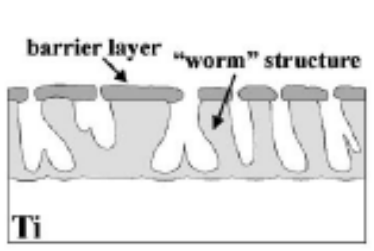

(c)

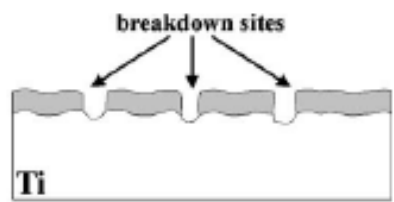

(b)

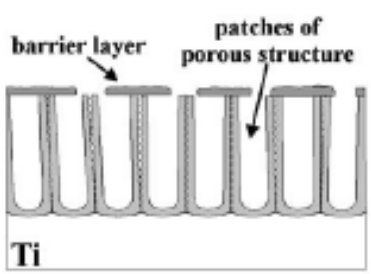

(d)

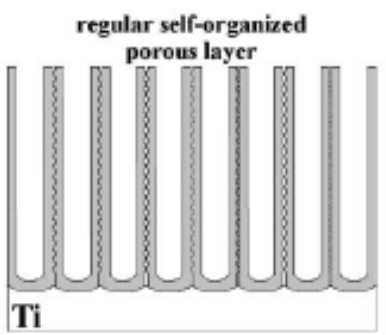

(e)

Figure 1-14. Schematic representation of the $\mathrm{TiO}_{2}$ nanotubular arrays formation: (a) compact layer growth to the maximum thickness, (b) breakdown of the barrier film, (c) growth of the pores resulting in a worm-like structure, (d) more ordered structure partially covered with the thin layer, and (e) regular self-organised porous $\mathrm{TiO}_{2}$ structure. (By Taveira et al, 2005) ${ }^{130}$ 
In this improved model, the chemical dissolution of titanium oxide was considered to be a dominant factor compared to the AAO case. And the dissolution rate of titanium oxide was found to greatly depend on the local acidification in fluoride containing electrolytes. Subsequently the $\mathrm{pH}$ gradient in the ATO tubes leads to an increase of the length of anodic nanotubes. Besides, the existence of titanium hydroxide during the formation of ATO was confirmed by an XPS study. However, this model still cannot explain the regular shape and ordering of the pores at the early stage of anodisation. In addition, it is also difficult to explain the formation of the gap between the nanotubes using this model.

\subsection{Other anodic metal oxides}

To date, there are a number of other metals have been tried to produce porous oxide films. Hexagonal porous anodic metal oxide structures have been achieved in some of these metals. The anodising ratio varies from $1.60 \mathrm{~nm} / \mathrm{V}$ to $2.20 \mathrm{~nm} / \mathrm{V}^{47}$

\subsubsection{Anodisation of hafnium}

Hafnium oxide has many interesting properties, e.g., its good chemical stability and thermal stability, high refractive index and relatively high dielectric constant. ${ }^{131,132}$ These properties make hafnium oxide a valuable material to be used as a protective coating, optical coating, gas sensor or capacitor. ${ }^{133-137}$ Self-organised porous hafnium oxide layers were obtained successfully for the first time by Tsuchiya and $\mathrm{Schmuki}^{31}$ via anodisation of hafnium at about $50 \mathrm{~V}$ in $1 \mathrm{M}$ $\mathrm{H}_{2} \mathrm{SO}_{4}+0.2 \mathrm{wt} \% \mathrm{NaF}$ at room temperature as shown in Figure 1-15. Anodisation potential was found to be a key factor affecting the morphology and the structure of the porous oxide. The pore diameter was found to increase with increasing potential. Porous hafnium oxide layers with high aspect ratios can be grown to a thickness of several tens micrometers. 


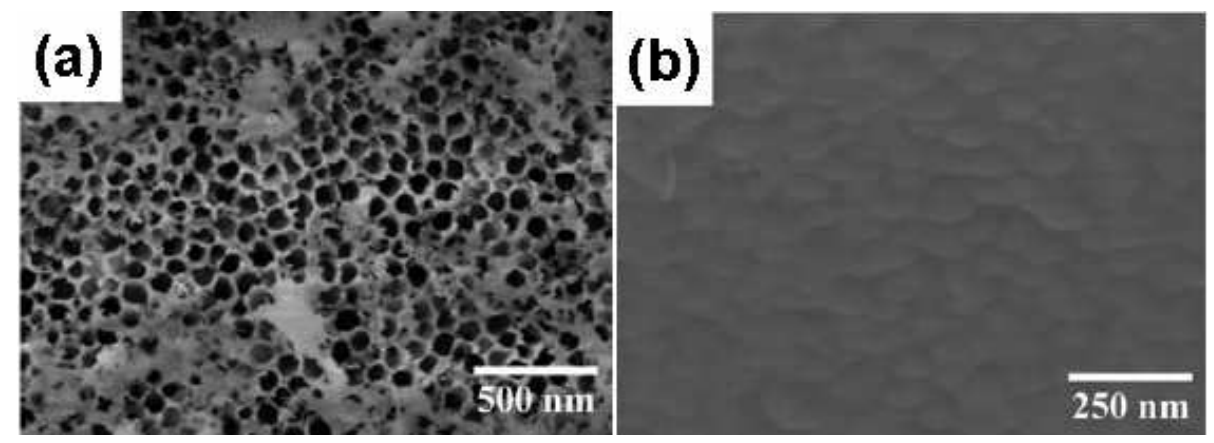

Figure 1-15. Plan-view (a) and bottom-view (b) FE-SEM images of anodic porous hafnium oxide layers formed at $50 \mathrm{~V}$ in $1 \mathrm{M} \mathrm{H}_{2} \mathrm{SO}_{4}+0.2 \mathrm{wt} \% \mathrm{NaF}$ at room temperature. (By Tsuchiya et $a l, 2005)^{31}$

\subsubsection{Anodisation of niobium}

Porous niobium oxide structures could be applied in gas sensors, ${ }^{138}$ catalysis, ${ }^{139}$ and optical ${ }^{140}$ and electrochromic ${ }^{141}$ devices. Anodisation of niobium has been studied in various electrolytes. ${ }^{142,143}$ Self-organised porous anodic niobium oxide films were successfully obtained by Sieber $e t a l^{32}$ and Karlinsey et $a l^{144}$ in $1 \mathrm{M} \mathrm{H}_{2} \mathrm{SO}_{4}+1 \mathrm{wt} \% \mathrm{HF}$ and $1.5 \% \mathrm{HF}$ respectively. More recently Choi et $a l^{145}$ obtained anodic $\mathrm{Nb}_{2} \mathrm{O}_{5}$ films with an effective thickness over $500 \mathrm{~nm}$, consisting of a protective outer layer of around 90-130 nm and an inner layer of 300-400 nm, via an optimized anodisation-annealing-anodisation process (Figure 1-16).

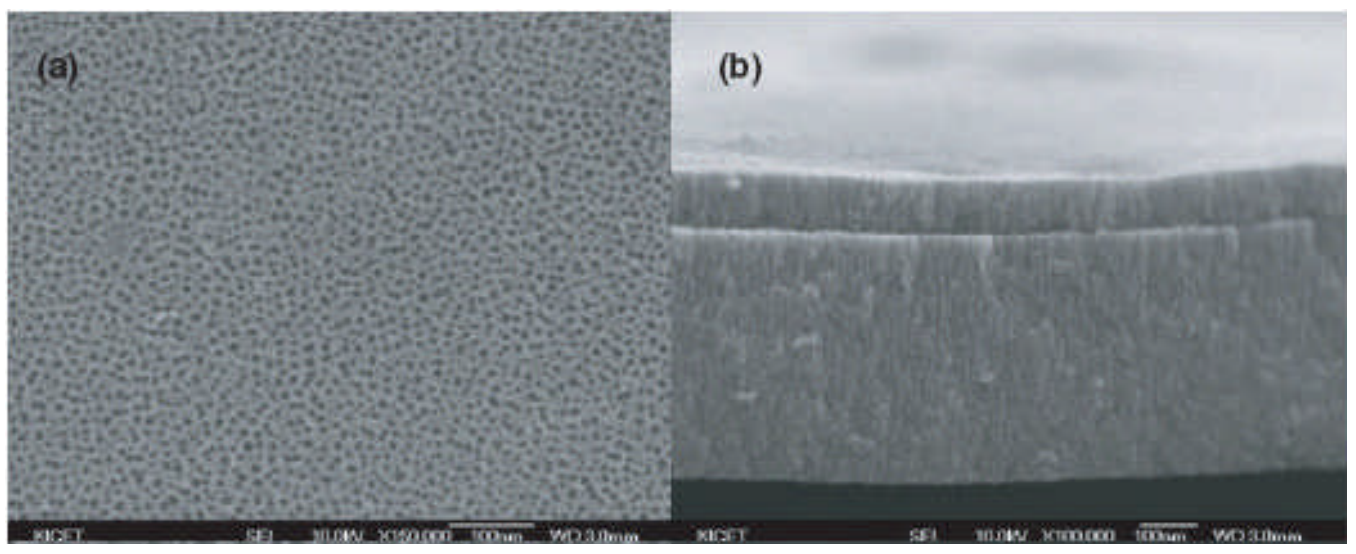

Figure 1-16. FE-SEM images showing top view (a) and sectional profile (b) of porous niobium oxides prepared in $1 \mathrm{wt} \% \mathrm{HF}+1 \mathrm{M} \mathrm{H}_{3} \mathrm{PO}_{4}$ by anodisation-annealing-anodisation process. (By Choi et al, 2007) $)^{145}$ (scale bar $100 \mathrm{~nm}$ ) 
A stress determined formation mechanism was proposed for anodisation of niobium by Zhao et al. ${ }^{146}$ Since the density of niobium in niobium pentoxide is much smaller than that of the niobium metal, oxidation of niobium metal yields an increase of inner stress in the oxide layer. As the inner stress increases, niobium oxide expands outside to form bulges and facilitates the oxidation of niobium metal under beneath, leading the formation of microcones. If no bulges are generated, the inner stress would break the oxide layer, leading to the growth of microcones.

\subsubsection{Anodisation of tantalum}

$\mathrm{Ta}_{2} \mathrm{O}_{5}$ has attracted intensive attention due to its application in optical devices, and as a protective coating material for chemical equipment, or suitable material for storage capacitors. ${ }^{147-151}$ Anodisation of tantalum has been widely investigated in sulfuric, phosphoric acid, and $\mathrm{Na}_{2} \mathrm{SO}_{4}$ solutions, and a layer of amorphous $\mathrm{Ta}_{2} \mathrm{O}_{5}$ with a uniform thickness could be obtained. ${ }^{143,152}$ Self-organised porous anodic tantalum oxide with a reasonably narrow size distribution was fabricated by Sieber $e t a l^{33,153}$ via anodising tantalum in $1 \mathrm{M} \mathrm{H}_{2} \mathrm{SO}_{4}+2$ wt $\%$ $\mathrm{HF}$ for $2 \mathrm{~h}$ after a potential ramp from open-circuit potential to $20 \mathrm{~V}$ with a sweep rate $100 \mathrm{mV} / \mathrm{s}$.
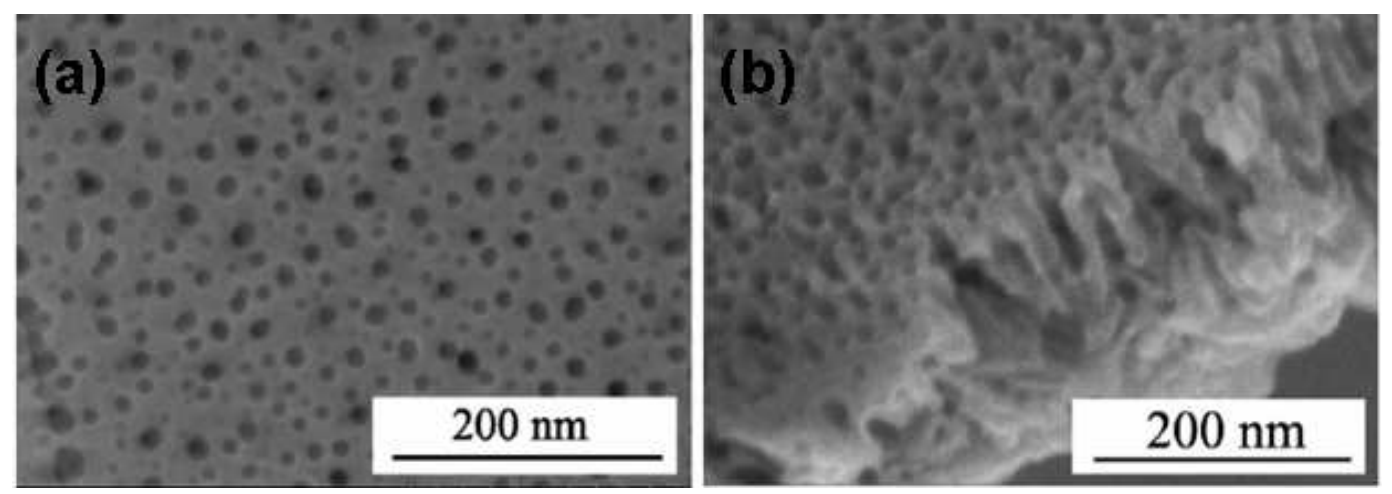

Figure 1-17. SEM top views (a) and cross sections (b) of anodic $\mathrm{Ta}_{2} \mathrm{O}_{5}$ prepared in $1 \mathrm{M} \mathrm{H}_{2} \mathrm{SO}_{4}+$ $2 \mathrm{wt} \% \mathrm{HF}$ for $2 \mathrm{~h}$ after a potential ramp from the open-circuit to $20 \mathrm{~V}$ with a sweep rate $100 \mathrm{mV}$ $\mathrm{s}^{-1}$. (By Sieber et al, 2005) $)^{153}$

\subsubsection{Anodisation of tungsten}

Tungsten oxide $\left(\mathrm{WO}_{3}\right)$ has been receiving considerable attention in recent years for its use in gas sensing, ${ }^{154,155}$ electrochromic ${ }^{156-162}$ and photochromic ${ }^{163,164}$ processes, etc. Based on early 
research in nonporous anodic tungsten oxide films, ${ }^{165-167}$ nanoporous anodic tungsten oxide was obtained by galvanostatic anodisation in oxalic acid by Mukherjee et $a l^{34}$, although the regularity of the pores appeared to be rather poor. Since then several groups have made a good progress to some extent in controlling the morphology and ordering of the anodic tungsten oxide. ${ }^{168-170}$ For example, de Tacconi et al ${ }^{170}$ reported that porous anodic $\mathrm{WO}_{3}$ made in $0.3 \mathrm{M}$ oxalic acid for $1 \mathrm{~h}$ at $35 \mathrm{~V}$ showed dense pores with a small pore size distribution as shown in Figure 1-18.

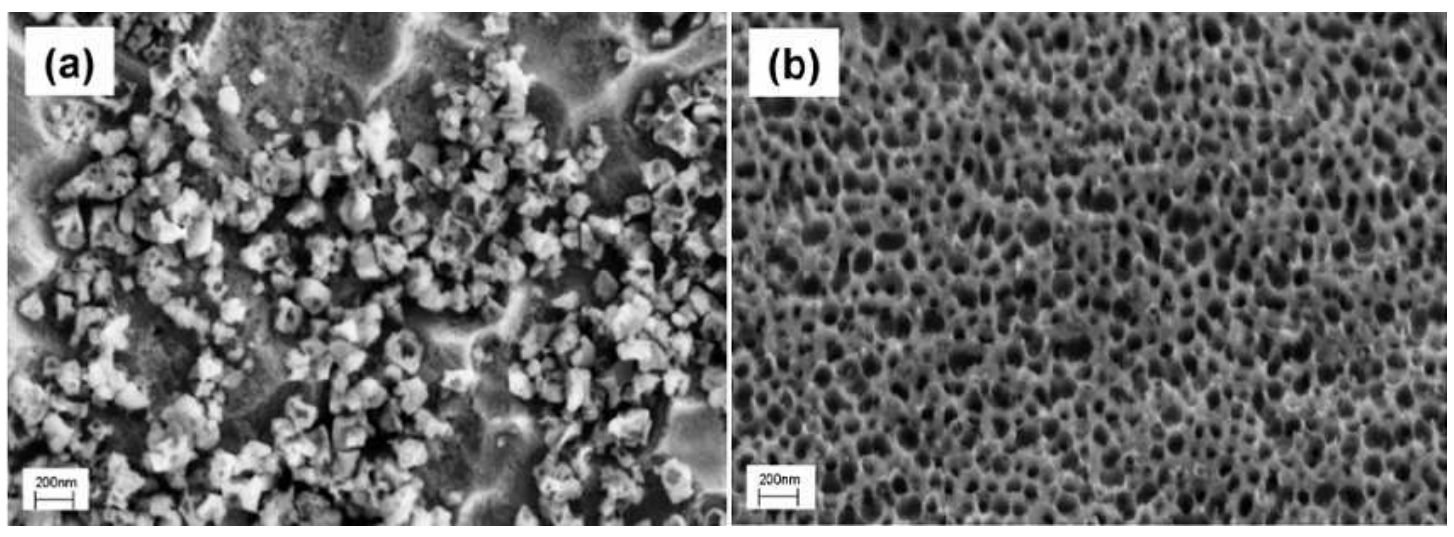

Figure 1-18. SEM image showing morphologies of nanoporous anodic $\mathrm{WO}_{3}$ films prepared in $0.3 \mathrm{M}$ oxalic acid for $1 \mathrm{~h}$ at (a) $10 \mathrm{~V}$, (b) $35 \mathrm{~V}$. (By De Tacconi et al, 2006) ${ }^{170}$

\subsubsection{Anodisation of vanadium}

Glacial acetic acid with small percentages of water and sodium tetraborate has been found to be the most suitable electrolyte for anodisation of vanadium. ${ }^{171-173}$ Due to the existence of the unfilled $d$ shell, the phase composition of the anodic vanadium oxide could be rather complicated, such as $\mathrm{V}_{2} \mathrm{O}_{5},{ }^{174-177} \mathrm{VO}_{2},{ }^{171,178,179}$ or a mixture of $\mathrm{V}_{2} \mathrm{O}_{5}$ and other oxide phases with lower oxidation states. ${ }^{177,180-182}$

\subsubsection{Anodisation of zirconium}

Zirconium oxide is an important functional material that plays a key role as an industrial catalysts and catalyst supports. ${ }^{183,184}$ It was reported that a compact anodic zirconium oxide layer 
of up to several hundred nanometers in thickness can be achieved in many electrolytes. ${ }^{185} \mathrm{~A}$ unique feature in comparison with other anodic metal oxides mentioned above is that the growth of the compact $\mathrm{ZrO}_{2}$ layer at room temperature directly leads to a crystalline film ${ }^{186}$ rather than an amorphous film as observed from other anodic metal oxides. Formation of self-organised porous zirconium oxide layers produced by anodisation of $\mathrm{Zr}$ at $30 \mathrm{~V}$ in an electrolyte of $1 \mathrm{M}$ $\mathrm{H}_{2} \mathrm{SO}_{4}+0.2 \mathrm{wt} \% \mathrm{NH}_{4} \mathrm{~F}$ was reported by Tsuchiya et al. ${ }^{36,187}$

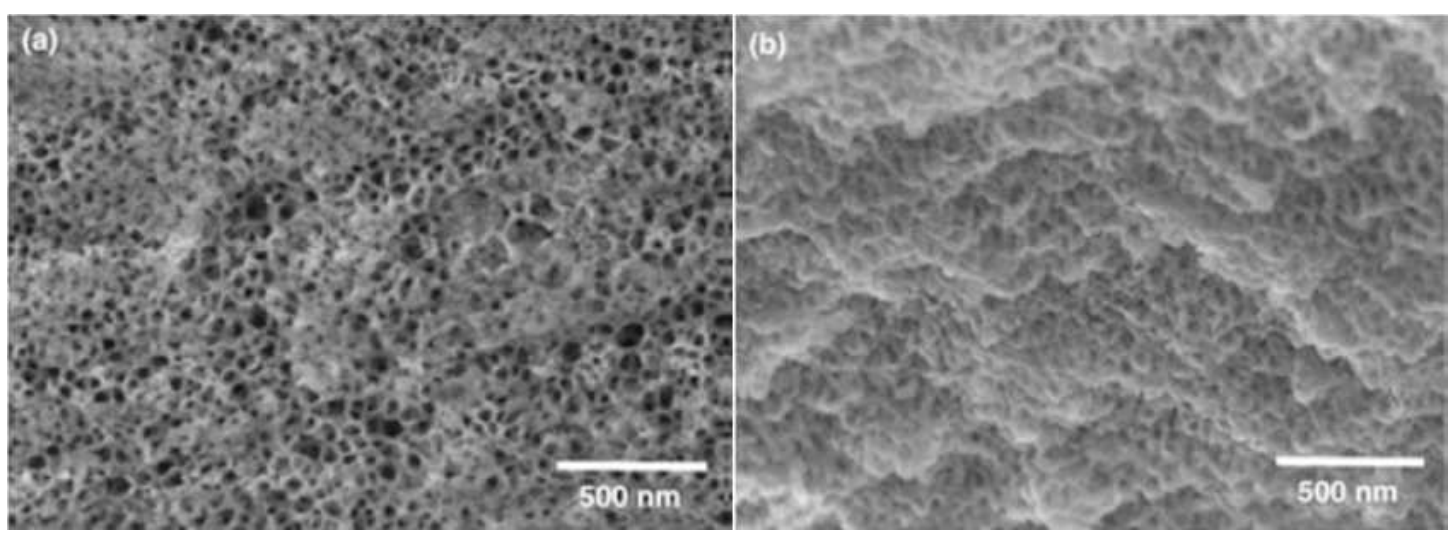

Figure 1-19. SEM images of anodic porous zirconium oxide layers. a) Plan view and b) cross-sectional view of the porous layer formed in $1 \mathrm{M} \mathrm{H}_{2} \mathrm{SO}_{4}+0.2 \mathrm{wt} \% \mathrm{NH}_{4} \mathrm{~F}$ electrolyte at 30 $\mathrm{V}$ for $5 \mathrm{~h}$ at $20^{\circ} \mathrm{C}$. (By Tsuchiya et al, 2005) ${ }^{36}$

\subsection{Applications of porous AAO and ATO}

As being the two most investigated anodic metal oxides, AAO nanopores films and ATO nanotubes arrays have been widely applied in laboratory research and commercial industry. Though in this dissertation we focus on the formation mechanism of self-organised highly ordered AAO and ATO anodic films, and the physical chemistry involved in the anodisation process, we would like to review some typical applications of AAO and ATO in this section.

\subsubsection{Application of porous anodic aluminium oxide}

Non-porous anodic alumina films have been widely applied in the prevention and decoration of aluminium surface, ${ }^{11}$ sealing, ${ }^{188-192}$ dyeing, ${ }^{193-195}$ and capacitors. ${ }^{12,196}$ In the recent years, 
self-organised porous AAO with perfect hexagonal pore patterns has been used to as a template to fabricate a variety of nanomaterials, such as nanoparticles, nanowires and nanotubes. ${ }^{14-19,71-74,197}$ Some typical applications of AAO include: etching masks for semiconductor substrate, ${ }^{14-16}$ pattern transfer by replica of AAO as a template, ${ }^{40}$ and deposition of functional materials by chemical vapor deposition (CVD) or electrochemical deposition method. ${ }^{17,18,198-203}$

\section{Etching mask for semiconductor substrate}

Pattern transfer of AAO nanochannels to a semiconductor substrate is promising for applications such as photonic band materials, field emitter arrays and quantum dot arrays. A typical method proposed by Shingubara et $a l^{16}$ uses a porous alumina film deposited directly on the semiconductor substrate. A thin porous AAO film with an aspect ratio below 5 was formed on a $\mathrm{Si} / \mathrm{SiO}_{2}$ substrate from sputtered aluminium. Reactive ion etching using chlorine with a high self-bias of RF plasma proved effective for pattern transfer to $\mathrm{Si}$.

\section{Pattern transfer by replica of porous alumina as a template}

Pattern transfer of AAO nanohole arrays to metallic hole arrays using a replica was firstly proposed by Masuda et $a l^{40}$ in 1995 . First, a negative nanohole array pattern was transferred to poly methyl methacrylate (PMMA) by coating it on the porous alumina film. Then, the porous AAO film was chemically wet-etched, leaving behind only the resist PMMA pattern, which can be used to form a replica by deposition of metals. Yanagishita et $a l^{204}$ reported a synthesis of Ni nanohole arrays using a two-step replication of AAO template (Figure 1-20).

An AAO film with through-holes was dipped in a methyl methacrylate (MMA) monomer with $5 \mathrm{wt} \%$ benzoyl peroxide as an initiator for polymerization. After polymerization by UV irradiation, the sample was polished to expose the two sides of the embedded AAO membrane. A thin Pt-Pd layer was coated onto the surface of the sample by sputtering, and then a $\mathrm{Au}$ reinforcing layer, which acted as an electrode for the metal deposition of hole array materials, was formed electrochemically. After the formation of the Au electrode, the alumina membrane was dissolved in $10 \mathrm{wt} \% \mathrm{NaOH}$ for $50 \mathrm{~h}$ to form the negative PMMA structures.

$\mathrm{Ni}$ nanohole arrays were fabricated by the electrochemical deposition of $\mathrm{Ni}$ into the cavities of 
the negative PMMA. A Ni hole array was obtained by dissolving the PMMA nanopillars in chloroform (Figure 1-20c).

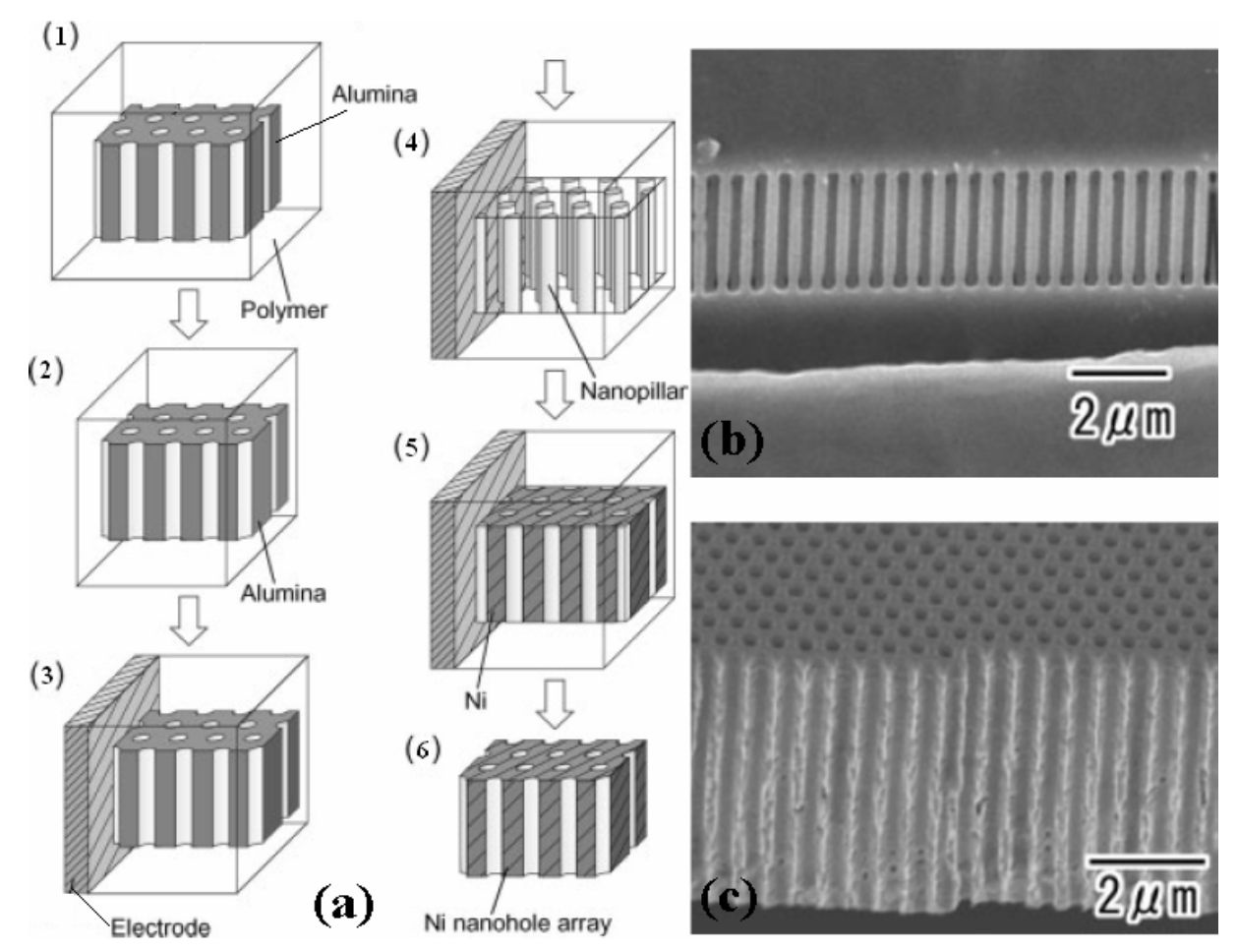

Figure 1-20. (a) Schematic for the preparation of metal nanohole arrays: 1) the AAO film is embedded in PMMA, 2) exposure of two sides of AAO, 3) formation of electrodes for electrochemical deposition of metal, 4) removal of AAO template, 5) electrochemical deposition of $\mathrm{Ni}$, and 6) removal of the PMMA negative. (b) SEM image of the PMMA nanopillar array after removing the alumina template. (c) Cross-sectional SEM image of a Ni hole array membrane. (By Yanagishita et al, 2005) ${ }^{204}$

\section{CVD deposition on AAO template}

Chemical vapor deposition (CVD) as a common method to produce high-purity, high-performance solid materials has been extensively used to synthesize nanomaterials in AAO templates in recent years. ${ }^{74,203}$

Silicon nanowires and nanotubes could be grown via the chemical vapor deposition in the AAO templates. The barrier layer of the AAO was etched in phosphoric acid at $30{ }^{\circ} \mathrm{C}$ for 90 min to get a through hole structure. Gold was deposited on one side of the AAO substrate using the 
magnetron sputtering method. The Au-deposited alumina substrate was placed into a tube furnace and the pressure in the furnace chamber was pumped down to $20 \mathrm{~Pa}$. A mixture of argon, hydrogen, and silane gases with a ratio of $10: 2: 1$ was allowed into the chamber. The pressure and temperature in the chamber were kept at $1450 \mathrm{~Pa}$ and $620{ }^{\circ} \mathrm{C}$ during deposition. In this way, silicon nanotubes could be produced. ${ }^{203}$

During the growth of silicon nanowires, the substrate was heated to a pre-set point of $500{ }^{\circ} \mathrm{C}$ before the mixed gases of argon, hydrogen, and silane with a ratio of $10: 20: 15$ were allowed into the chamber. The temperature and pressure were kept constant at $500{ }^{\circ} \mathrm{C}$ and $1400 \mathrm{~Pa}$ respectively during the deposition. ${ }^{74}$

Both the silicon nanotubes and nanowires grew on the vapor-liquid-solid (VLS) mechanism with gold as the catalyst, and silane as the vapor silicon source. Figure 1-21 shows the morphologies of the products in TEM characterization.
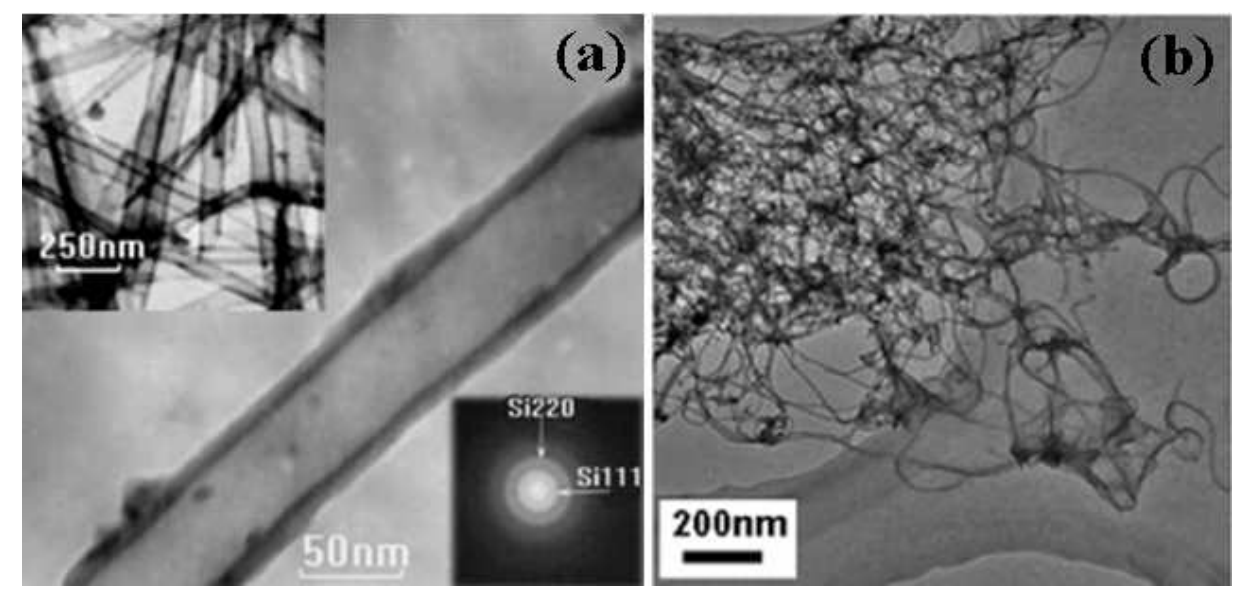

Figure 1-21. TEM images of silicon nanotubes, (By Sha et al, 2002) ${ }^{203}$ and silicon nanowires (By Su et al, 2006) ${ }^{74}$ synthesized by CVD method using AAO as a template.

Carbon nanotubes in porous anodic alumina have been intensively studied by several groups recently. It is well known that the CVD growth of carbon nanotubes needs catalysis for thermal decomposition of precursors. As one example, Figure 1-22 shows a typical fabrication process of a carbon nanotubular array by using a highly ordered AAO film as a template by Li et al. ${ }^{17}$ The precursor cobalt was electrodeposited into the AAO channels as catalyst. Then the carbon nanotubes grew via the pyrolysis of $\mathrm{C}_{2} \mathrm{H}_{2}$ in the CVD chamber. 

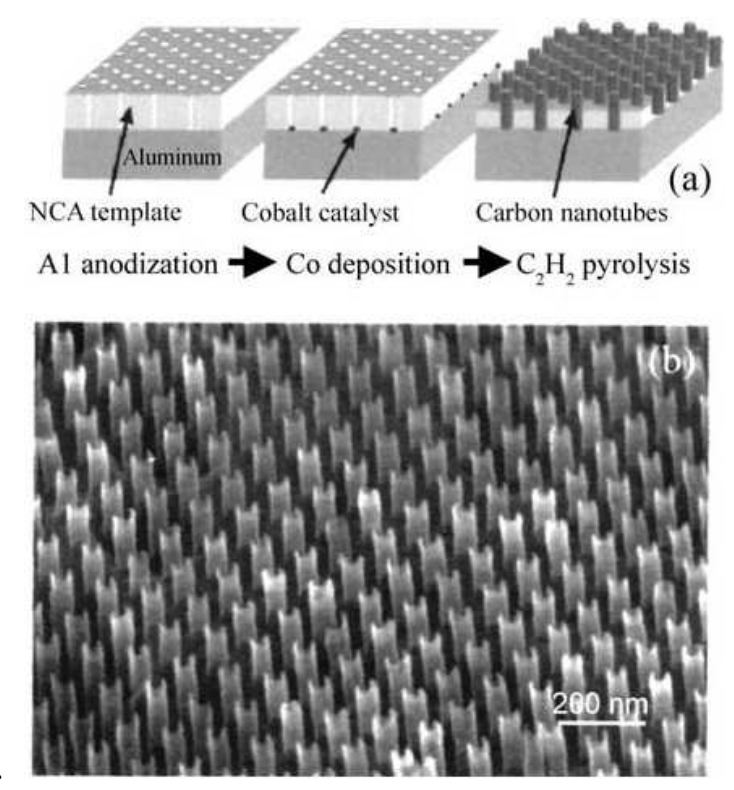

Figure 1-22. (a) Schematic of fabrication process of hexagonally ordered array of carbon nanotubes and (b) the corresponding SEM image of the product. (By Li et al, 1999) ${ }^{17}$

\section{Electrochemical deposition on AAO template}

AAO templates have been widely used to study arrays of ferromagnetic nanowires since the simplicity to control parameters such as length, wire diameter, or interwire distance makes them an ideal playground to study a range of magnetic phenomena. The technique to synthesize ferromagnetic nanowires by electrodeposition assisted by AAO template is inexpensive and convenient. Using the AAO as a template, Qin $e t a l^{205}$ fabricated $\mathrm{Fe}, \mathrm{Co}$, Ni nanowires by a typical electrodeposition method:

To perform the metal electrodeposition inside AAO channels, one side of the AAO membrane was coated by a thin layer of DC-sputtered gold as counter electrode. Nanowires were grown inside the AAO channels by a potentiostatic electrochemical deposition procedure. A mixed electrolyte of $\mathrm{NiSO}_{4} \cdot 6 \mathrm{H}_{2} \mathrm{O}, 300 \mathrm{~g} / \mathrm{L} ; \mathrm{NiCl}_{2} \cdot 6 \mathrm{H}_{2} \mathrm{O}, 45 \mathrm{~g} / \mathrm{L} ; \mathrm{H}_{3} \mathrm{BO}_{3}, 45 \mathrm{~g} / \mathrm{L}$ was used to fabricate $\mathrm{Ni}$ nanowires. The electrolysis was carried out at $25^{\circ} \mathrm{C}, 2.0 \mathrm{~V}_{\mathrm{DC}}$ with a $\mathrm{pH}$ value of 4.0 . Co nanowires were deposited using a solution with a mixture of $140 \mathrm{~g} / \mathrm{L} \mathrm{CoSO}$ and $50 \mathrm{~g} / \mathrm{L} \mathrm{H}_{3} \mathrm{BO}_{3}$ at $25{ }^{\circ} \mathrm{C}, 2.5 \mathrm{~V}_{\mathrm{DC}}$ with a $\mathrm{pH}$ value of 4.0. Fe nanowires were electrodeposited by using an electrolyte containing $120 \mathrm{~g} / \mathrm{L} \mathrm{FeSO} \cdot 7 \mathrm{H}_{2} \mathrm{O}$, a small amount of Fe-enriched $\mathrm{FeCl}_{3}, 45 \mathrm{~g} / \mathrm{L} \mathrm{H}_{3} \mathrm{BO}_{3}$, 
and $1 \mathrm{~g} / \mathrm{L}$ ascorbic acid at $25^{\circ} \mathrm{C}, 2.0 \mathrm{~V}_{\mathrm{DC}}$ with a $\mathrm{pH}$ value of 2.0 .

After the electrodeposition, AAO films with metal wires embedded inside were carefully cleaned by acetone for further characterization. Figure 1-23 shows the SEM image of Co nanowires and the XRD patterns of the prepared Fe, Co, Ni nanowires.

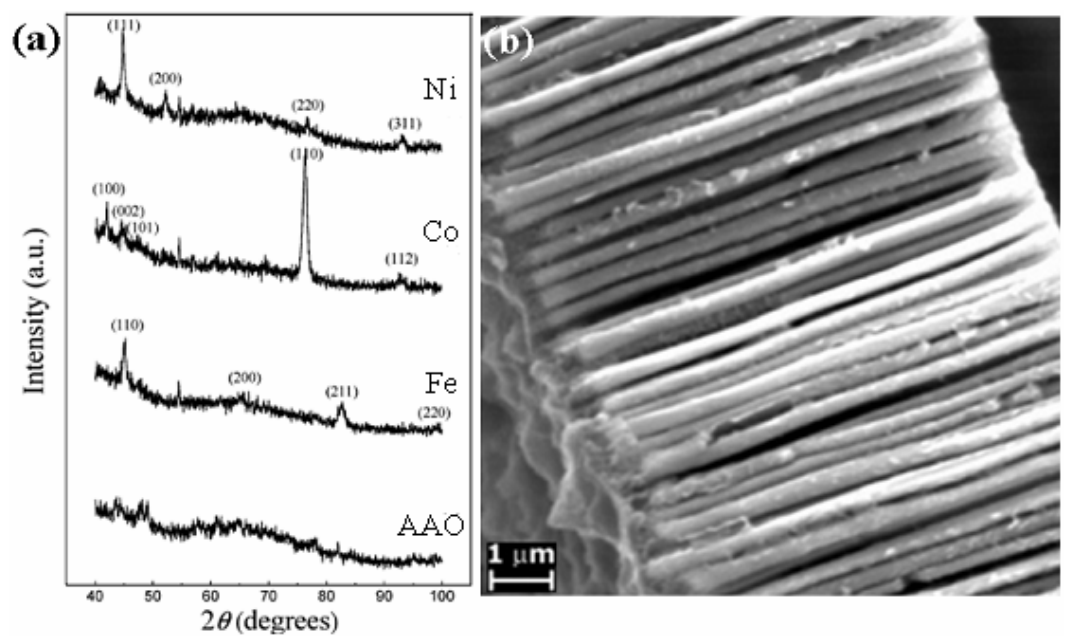

Figure 1-23. XRD patterns of AAO, and the prepared Fe, Co, Ni nanowires (a), and the SEM image of Co nanowires (b). (By Qin et al, 2005) 205

\subsubsection{Application of porous anodic titanium oxide}

$\mathrm{TiO}_{2}$ is an n-type semiconductor with a band gap energy of $3.2 \mathrm{eV}$ for anatase, $3.0 \mathrm{eV}$ for rutile, or 3.2-3.5 eV in its amorphous state. Based on its semiconductor nature, $\mathrm{TiO}_{2}$ was widely applied in photoelectrochemical and water photolysis, dye-sensitized solar cells, hydrogen sensing and in self-cleaning sensors. In many of these applications the ATO nanotubular structure can bring specific advantages as reviewed by Mor et al ${ }^{129}$ and Ghicov et al. ${ }^{206}$

\section{ATO in photocatalyst and water photolysis}

$\mathrm{TiO}_{2}$ has excellent photocatalytic properties based on the fact that the valence band position allows photogenerated holes to react with the surrounding water to form $\mathrm{OH}^{-}$radicals. These in turn decompose essentially any organic material in the surrounding to $\mathrm{H}_{2} \mathrm{O}+\mathrm{CO}_{2}$. It is well established that the presence of oxygen and water play a crucial role in the photocatalytic 
cleaning of titania, hence the sensor was exposed to air during UV exposure to facilitate removal of the oil from the sensor surface.

In fact, it should be noted that UV exposure with the sensor kept in a nitrogen atmosphere did not result in sensor recovery. If the relevant redox potential of the contaminant does not lie within the band gap of titania, the organic contaminants cannot be oxidized by photogenerated electron-hole pairs on the surface of titania. However, because the potential of water and oxygen exist within the band gap of titania, the photogenerated holes in the valence band can oxidize water to produce highly reactive hydroxyl radicals $(\cdot \mathrm{OH})$, and the photogenerated electrons in the conduction band can reduce oxygen to form highly reactive superoxide $\left(\mathrm{O}_{2}{ }^{-}\right)$ions [eqs (1.10)-(1.12)], which then assist in oxidizing the organic species. ${ }^{103}$

$$
\begin{aligned}
& \mathrm{TiO}_{2}+h v \rightarrow h^{+}+e^{-} \\
& \mathrm{H}_{2} \mathrm{O}+h^{+} \rightarrow \mathrm{OH}+\mathrm{H}^{+} \\
& \mathrm{O}_{2}+e^{-} \rightarrow \mathrm{O}_{2}
\end{aligned}
$$

In a size-quantized semiconductor system, Wang et $a l^{207}$ reported an increase in photocatalytic activity with a decrease in titania particle-size from $21 \mathrm{~nm}$ to $11 \mathrm{~nm}$; however, the photocatalytic activity decreased when the size was further reduced to $6 \mathrm{~nm}$. The results indicate that, depending on the nanoarchitecture, there exists an optimal particle size in nanocrystalline $\mathrm{TiO}_{2}$ for maximum photocatalytic activity. Zhang et $a l^{208}$ explained these observations by correlating an increase in rate of interfacial charge carrier transfer with decrease in particle size; on reaching a certain limiting particle size, surface recombination exceeds the interfacial charge transfer process and therefore the photocatalytic activity reduced.

Recently it has been shown that nanotube layers can be more efficient photocatalysts than comparable nanoparticles layers. ${ }^{209}$ The tubular $\mathrm{TiO}_{2}$ provides a more optimized geometry with significantly shorter carrier-diffusion paths in the tube walls, including lower trapping and recombination kinetics of light-generated electron-hole pairs in comparison with nanoparticles, where these pairs have to travel between single nanoparticles. 


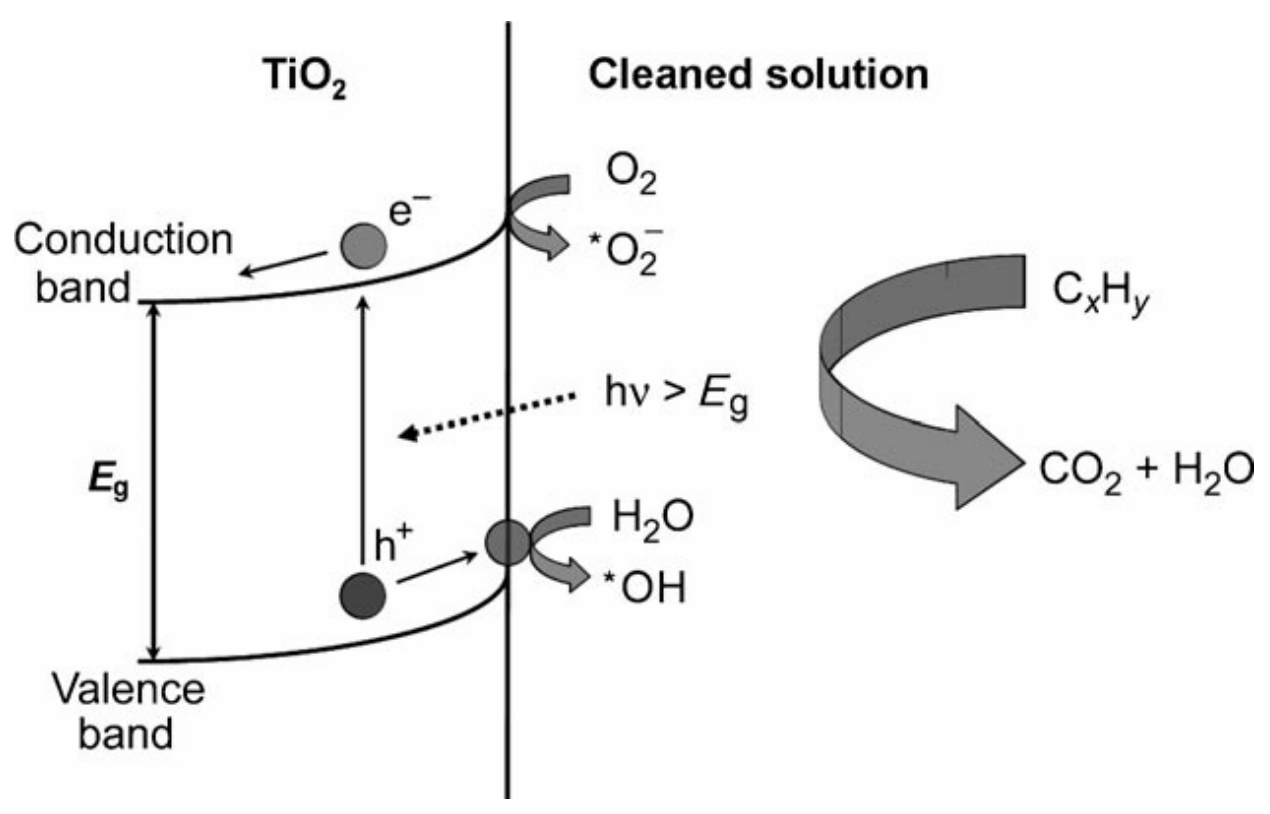

Figure 1-24. Principles of photocatalytic decomposition on semiconductor $\mathrm{TiO}_{2}$ surfaces. Photons excite electrons from the valence band to a conduction band forming an electron-hole $\left(\mathrm{e}^{-}-\mathrm{h}^{+}\right)$pair. This charge carrier can recombine in bulk or migrate to the surface and react with the adsorbed species, which leads to their decomposition by direct oxidation on the holes, or by $\mathrm{HO}^{\prime}$ and $\mathrm{O}_{2}^{-}$radicals. (By Macak et al, 2007) ${ }^{209}$

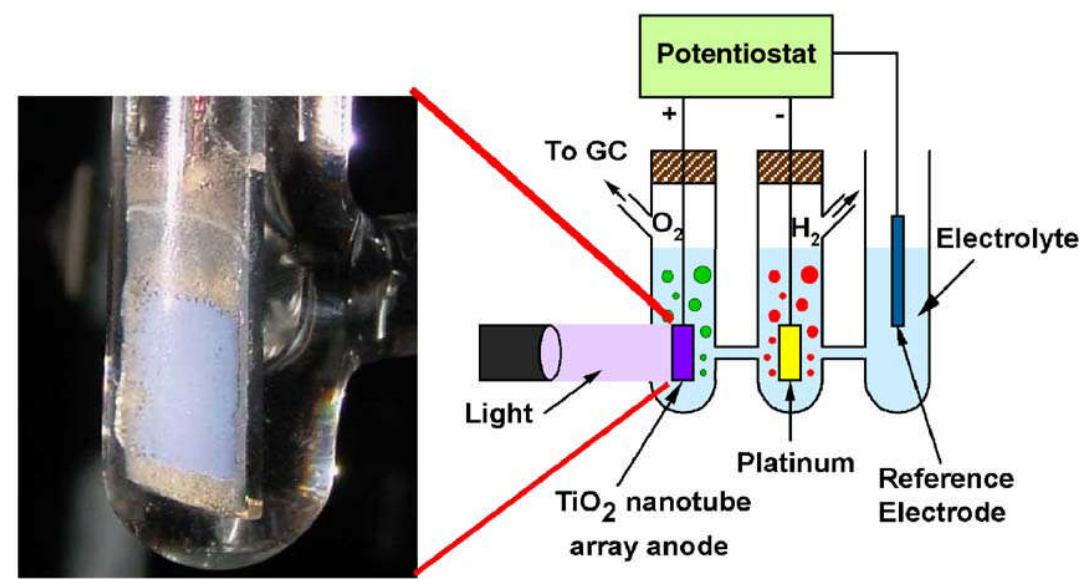

Figure 1-25. Illustrative drawing of experimental setup for water photoelectrolysis. (By Mor et al, 2006) $)^{129}$

Figure 1-25 is an illustrative drawing of the experimental setup for water photoelectrolysis measurements with the nanotube arrays used as the photoanodes from which oxygen is evolved. As reported by Paulose $e t a l^{30}$, a typical $45 \mu \mathrm{m}$ long nanotube-array sample annealed at $550{ }^{\circ} \mathrm{C}$ in dry oxygen, in a $1 \mathrm{M} \mathrm{KOH}$ solution under 320-400 nm UV illumination, $100 \mathrm{~mW} / \mathrm{cm}^{2}$, 
exhibited a remarkable water photoelectrolysis photoconversion efficiency of $16.25 \%$.

The photoconversion efficiency, $\eta$, is calculated as: $:^{210}$

$$
\begin{aligned}
\eta(\%) & =[(\text { total power output }- \text { electrical power output }) / \text { light power input }] \times 100 \\
& =j_{\mathrm{p}}\left[\left(E_{\text {rev }}{ }^{\circ}-\left|E_{\text {app }}\right|\right) / I_{0}\right] \times 100
\end{aligned}
$$

where $j_{\mathrm{p}}$ is the photocurrent density $\left(\mathrm{mW} / \mathrm{cm}^{2}\right), j_{\mathrm{p}} E_{\text {rev }}{ }^{\circ}$ is the total power output, $j_{\mathrm{p}}\left|E_{\text {app }}\right|$ is the electrical power input, and $I_{0}$ is the power density of incident light $\left(\mathrm{mW} / \mathrm{cm}^{2}\right) . E_{\mathrm{rev}}{ }^{\circ}$ is the standard reversible potential which is $1.23 \mathrm{~V} / \mathrm{NHE}$, and the applied potential is $E_{\text {app }}=E_{\text {meas }}-E_{\text {aoc }}$, where $E_{\text {meas }}$ is the electrode potential (vs $\mathrm{Ag} / \mathrm{AgCl}$ ) of the working electrode at which photocurrent was measured under illumination and $E_{\mathrm{aoc}}$ is the electrode potential (vs $\mathrm{Ag} / \mathrm{AgCl}$ ) of the same working electrode under open circuit conditions, under the same illumination, and in the same electrolyte. The voltage at which the photocurrent becomes zero was taken as $E_{\mathrm{aoc}}$.

\section{ATO in hydrogen sensing}

Hydrogen sensors can be viewed as one of many enabling steps on the path to a hydrogen economy and thus are of great scientific and practical importance. Several studies have shown that the highly ordered ATO nanotube arrays demonstrate remarkable changes in electrical resistance in the presence of hydrogen gas ${ }^{102-105,119}$ with the hydrogen sensitivity more prominent for smaller diameter and thin-walled nanotubes. Oxygen in air may be chemisorbed in the form of $\mathrm{O}_{2}$ on the nanotube surface by trapping electrons from its conduction band leading to an enhanced base resistance. ${ }^{21,212}$ Hydrogen can remove this chemisorbed oxygen thus reducing the resistance.

Figure 1-26 shows the variation in resistance of a short $22 \mathrm{~nm}$ diameter ATO nanotube sample at room temperature when the hydrogen concentration is cycled in discrete ppm steps. It is clear from Figure 1-26 that the nanotubes respond without hysteresis, with sensor resistance decreasing to a few tens of ohms on exposure to $1000 \mathrm{ppm}$ hydrogen.

Varghese $e t a l^{204}$ and Paulose et $a l^{105}$ reported a transcutaneous hydrogen gas sensor of unprecedented sensitivity as a diagnostic tool for determining lactose intolerance due to lactase deficiency. This hydrogen gas sensor, based on the use of highly-ordered titania nanotubular arrays made by anodisation of a $250 \mu \mathrm{m}$ thick titanium foil, shows a remarkable change in electrical resistance of 8.7 orders of magnitude when cycled between air and nitrogen containing 1000 ppm of hydrogen. 


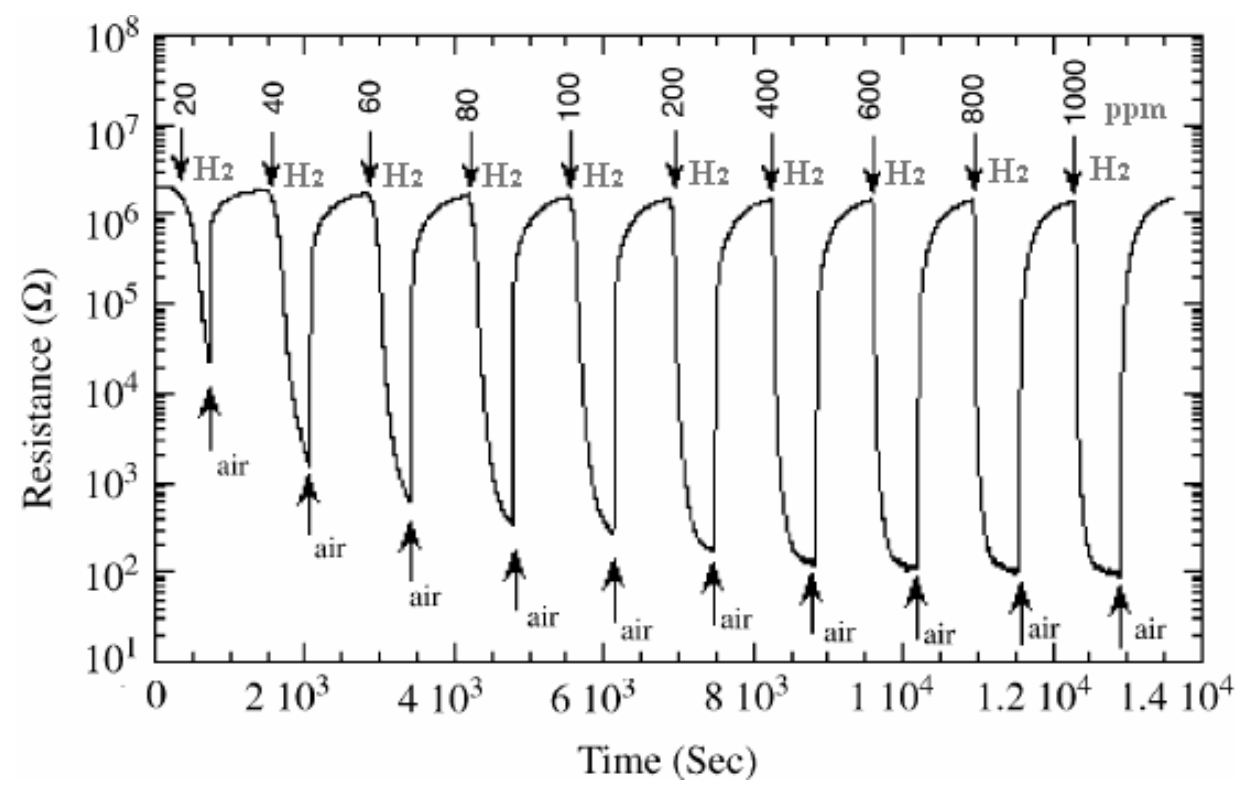

Figure 1-26. Electrical resistance of $22 \mathrm{~nm}$ diameter $360 \mathrm{~nm}$ long $\mathrm{TiO}_{2}$ nanotube array when exposed to different hydrogen concentrations at room temperature. The nanotube response is completely reversible without hysteresis or drift. (By Mor et al, 2006) $)^{129}$

\section{ATO in Dye-sensitized solar cells (DSSCs)}

One of the widest uses of $\mathrm{TiO}_{2}$ in functional applications is in dye-sensitized solar cells. In 1985 Desilvestro et $a l^{213}$ showed that the light to electricity conversion efficiency of the dye sensitized $\mathrm{TiO}_{2}$ can be drastically enhanced if the latter is used in a nanoparticulate form. Several approaches have been proposed to use nanostructured electrodes other than $\mathrm{TiO}_{2}$, including $\mathrm{ZnO}$ or $\mathrm{SnO}_{2}$ in DSSCs. But up to now the most efficient DSSCs are still based on $\mathrm{TiO}_{2}$ electrodes. ${ }^{206}$

Figure 1-27 illustrates typical front-side and back-side illuminated dye solar cell integrations. Light enters the cell through the transparent electrode. Photons striking the dye with enough energy to be absorbed will produce an excited state of the dye, from which an electron can be injected into the conduction band of the $\mathrm{TiO}_{2}$. Then it moves by diffusion to the anode. Meanwhile, the dye molecule has lost an electron and the molecule will decompose if no another electron provided. The dye strips one from iodide in electrolyte below the $\mathrm{TiO}_{2}$, oxidizing it into triiodide. This reaction occurs quite quickly compared to the time that it takes for the injected electron to recombine with the oxidized dye molecule, preventing this recombination reaction. The triiodide then recovers its missing electron by mechanically diffusing to the bottom of the cell, where the counter electrode re-introduces the electrons after flowing through the external 
circuit.

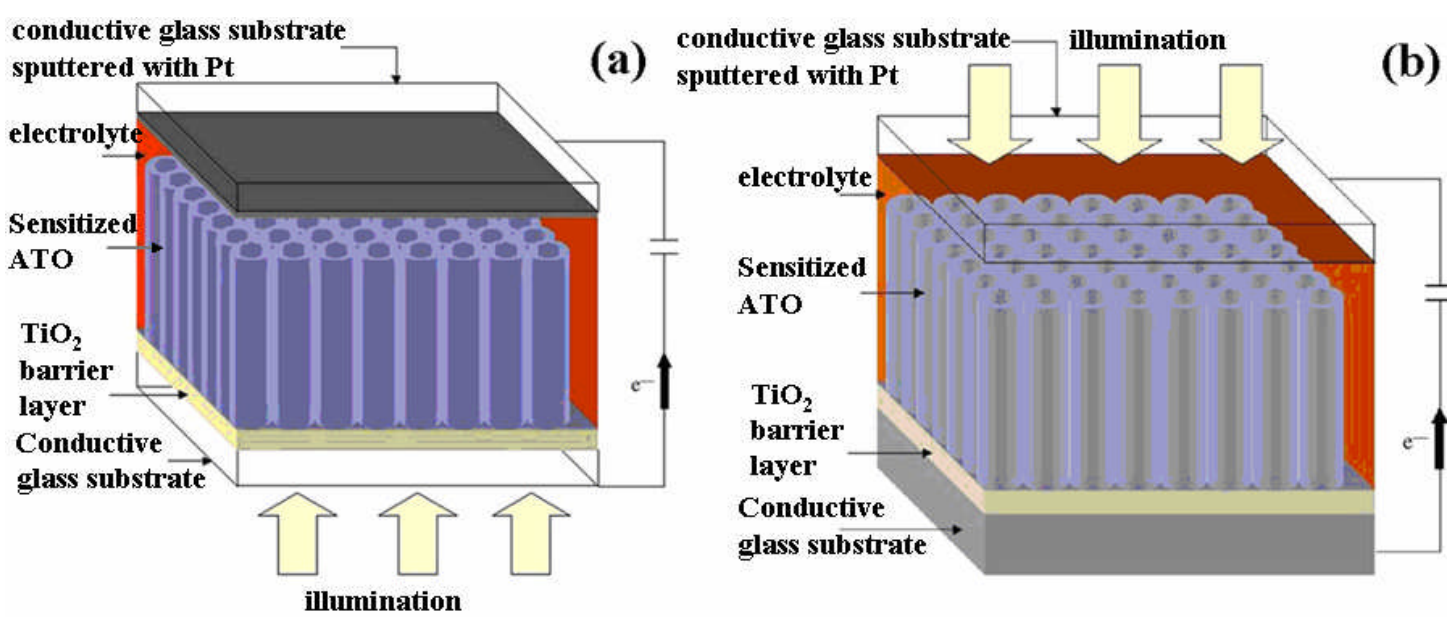

Figure 1-27. Schematic diagrams of front-side (a) and back-side (b) illuminated dye solar cell structures. (By Mor et al, 2006) ${ }^{129}$

Studies on the electronic transport properties have shown that in a nanoparticulate film like $\mathrm{TiO}_{2}$, the transport of the charge carriers can be extremely slow. Therefore the probability for recombination to occur before the charge carrier can be collected in the external circuit is comparably high. This is believed to be one of the key limiting mechanisms in dye-sensitized solar cells (DSSCs) based on $\mathrm{TiO}_{2}$ nanoparticles, one of the main reasons why a nanotubular arrangement could be advantageous.

Nanotubular $\mathrm{TiO}_{2}$ layers are expected to behave differently from nanoparticulate $\mathrm{TiO}_{2}$ layers due to: (a) a much lower degree of distorted crystal structures at grain boundaries that enhance electron scattering (reducing the electron mobility); (b) smooth tube walls that may provide a lower surface state density — thus reducing surface state recombination effects; (c) suppression of an extensive random walk path through a porous network due to the $1 \mathrm{D}$ nature of the tubes. ${ }^{206}$

Recently, a number of studies demonstrated that tube length and radius are crucial parameters to increase the light conversion efficiency. A light to electricity photoconversion efficiency of $6.9 \%$ was achieved by Shankar et $a l^{124}$ using very long nanotubular arrays up to $220 \mu \mathrm{m}$ in backside illuminated dye-sensitized solar cells. 


\section{ATO in self-cleaning sensor}

Combination of the hydrogen gas sensing properties and photocatalytic properties of the $\mathrm{TiO}_{2}$ nanotube arrays gives rise to the interesting application of self-cleaning sensors; that is sensors able to use the ambient light to recover from detrimental contamination.

$\mathrm{TiO}_{2}$ is an n-type semiconductor; with ultraviolet photon absorption, an electron-hole pair is generated that can facilitate reduction and oxidation chemistry at the surface of the material. These redox reactions clean the surface by breaking down organic contaminants to form mainly $\mathrm{CO}_{2}$ and $\mathrm{H}_{2} \mathrm{O}{ }^{214}$ The ATO nanotube room temperature hydrogen gas sensor is able to self-clean with exposure to UV light, fully recovering initial properties after being contaminated by either motor oil and/or stearic acid. ${ }^{119}$ The self-cleaning properties are demonstrated by observing the sensor base resistance, and the resistance change upon exposure to a $1000 \mathrm{ppm}$ hydrogen atmosphere, resistance with contamination and after self cleaning. Figure 1-28 shows that the sensitivity of the sensor, comprised of an array of $22 \mathrm{~nm}$ inner-diameter ATO nanotubes $200 \mathrm{~nm}$ in length recovered fully after self-cleaning the contaminants under exposure to a $270 \mathrm{~mW} / \mathrm{cm}^{2}$ UV light of mixed (365 nm, $254 \mathrm{~nm}$ ) wavelength.

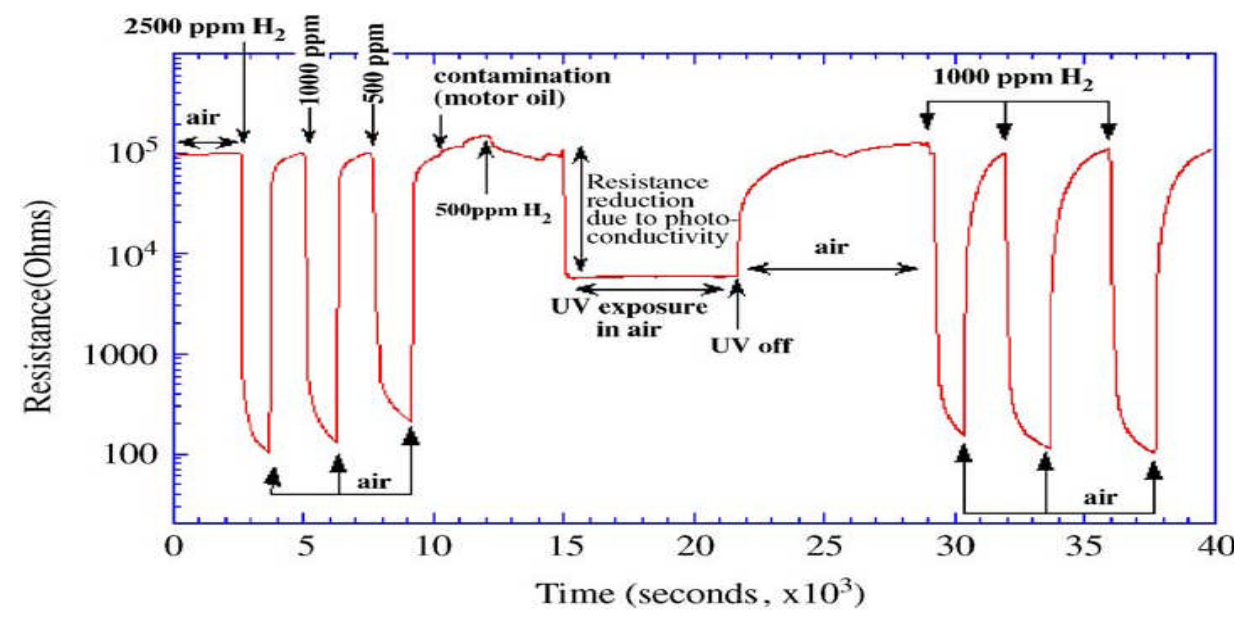

Figure 1-28. Resistance variation before, during and after sensor contamination with motor shows the ATO hydrogen sensor regain its original sensitivity under UV exposure. (By Mor et al, 2003, 2006) $)^{119,129}$

\section{ATO in free-standing flow through membranes}

By opening the closed end of the nanotubes, a free-standing ATO nanotubular membrane 
could be fabricated. ${ }^{215}$ The diameter of the nanotubular membrane is narrowly distributed and can be controlled by varying the anodising voltage and electrolyte, which could be used for filtering nanoparticles of different size. Besides, this through hole free-standing ATO nanotubular membrane has direct photocatalytic applications. Figure 1-29b shows a two compartment cell divided by the ATO membrane. One compartment was filled with methylene blue (MB) while the other contained colorless distilled water. After illuminating the membrane from the MB compartment side with UV light, the MB can be entirely decomposed before diffusing across the ATO membrane due to the fast photocatalytic decomposition rate.
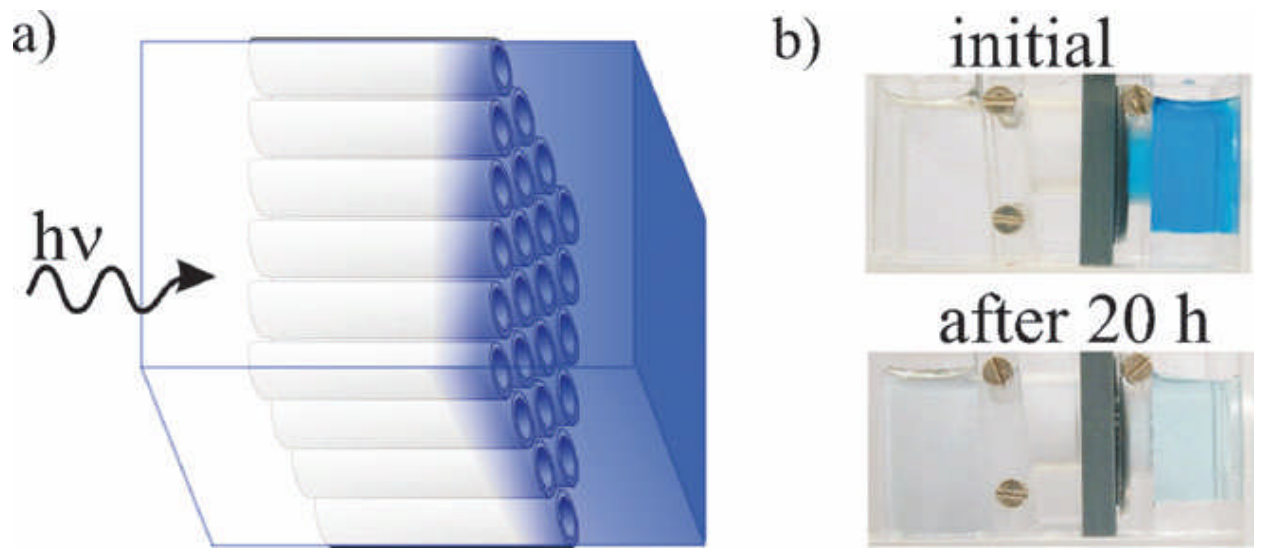

Figure 1-29. Schematic representation of methylene blue degradation using a $\mathrm{TiO}_{2}$ nanotubular membrane (a). Photographs from the two compartment cell (left side filled with pure water, right side with methylene blue in water) separated by a $\mathrm{TiO}_{2}$ nanotube membrane in the initial stage and after $20 \mathrm{~h} \mathrm{UV} \mathrm{light} \mathrm{(325} \mathrm{nm)} \mathrm{illumination} \mathrm{from} \mathrm{the} \mathrm{right} \mathrm{side} \mathrm{(b).} \mathrm{(By} \mathrm{Ghicov} \mathrm{et} \mathrm{al,} \mathrm{2009,}$ Albu et al, 2007) 206,215

\section{Biomedical applications}

One of the key features of $\mathrm{TiO}_{2}$ interacting with body fluids is that it stimulates hydroxyapatite growth. ${ }^{206}$ In the case of bone implants, apatite formation is important for osseointegration. Tsuchiya et $a l^{216}$ showed that on nanotubular $\mathrm{TiO}_{2}$ surfaces hydroxyapatite growth can be enhanced, in particular a faster and denser apatite precipitation can be observed on $\mathrm{TiO}_{2}$ nanotube surfaces. Furthermore, annealing of the as prepared ATO to anatase phase could strongly enhance the growth process of hydroxyapatite. 


\subsection{Aims and Objectives}

The principal aims of this project are to prepare ordered porous aluminium and titanium oxides films via anodisation of aluminium and titanium metal foil, and to investigate the physical and chemical mechanisms in the formation of these anodic metal oxide films. There are several objectives as follows.

1. To study the pore initiation origin in the anodisation of aluminium and titanium, and explain the formation of hemispherical pore bottom geometry. To study the variation of the anodic ratio of barrier layer thickness with increasing applied voltage, and explain the difference in the anodic ratio of barrier layer thickness in different electrolytes.

2. To establish an ideal model to interpret the self-organisation growth mechanism of the AAO pore arrays and ATO nanotubes: from the single pore growth to the adjustment of neighboring pores and finally to the highly ordered anodic oxide films. As well as the adjusting model when the anodic pores grow in depth, the driven force for the initial pore ordering at the early stage of anodisation also needs to be clarified.

3. To study the effects of the anodisation conditions, including solution type and applied voltage, on the structure of the anodic metal oxides. The relationship between the current density and the thickness of the barrier layer for AAO and ATO, and the relationship between the current density and the porosity for AAO and ATO in a particular electrolyte and given temperature need to be established. The dependence of current density and subsequently the dependences of porosities for AAO and ATO films on applied voltage in given electrolyte and temperature were also under investigation.

4. To study the physical chemistry in the anodisation processes of $\mathrm{Al}$ and $\mathrm{Ti}$. Water dissociation at the electrolyte/oxide interface was found to greatly affect the structure of the anodic oxide films. The relative dissociation rate of water compared to the dissolution rate of oxide determines the porosity of the anodic oxide films. A computational chemistry method was used to measure the electric field enhanced effect on the dissociation rate of water in anodisation of metal. Finally to find out a reasonable and practical way to control the structural formation of AAO and ATO more precisely. 


\section{Experiments}

\subsection{Anodisation method}

The working unit of anodisation is home-made. Figure 2-1 shows an apparatus for our electrochemical (anodisation) experiments. The electrochemical cell consists of a conventional two-electrode system, i.e., a graphite piece acting as the cathode and a metal foil acting as the working anode. The graphite cathode is connected to the negative terminal of a high-voltage Consort EV243 DC power supply. The metal foil is pressed against a $\mathrm{Cu}$ plate connecting to the positive terminal of the power supply. The surface area of the metal foil exposed to the bath electrolyte is about $2 \mathrm{~cm}^{2}$.

As is known, barrier oxide grows in near neutral solutions in which the metal oxide is hardly soluble. Porous oxide grows in acidic electrolyte in which oxide can not only be deposited but also dissolves. In the present work, the bath electrolytes selected for the growth of porous AAO included $0.3 \mathrm{M}$ oxalic acid and $2 \mathrm{wt} \%$ phosphorus acid. While to grow ATO porous arrays, ethylene glycol containing $0.3 \mathrm{wt} \% \mathrm{NH}_{4} \mathrm{~F}$ and $2 \mathrm{wt} \%$ water was chosen as the electrolyte.

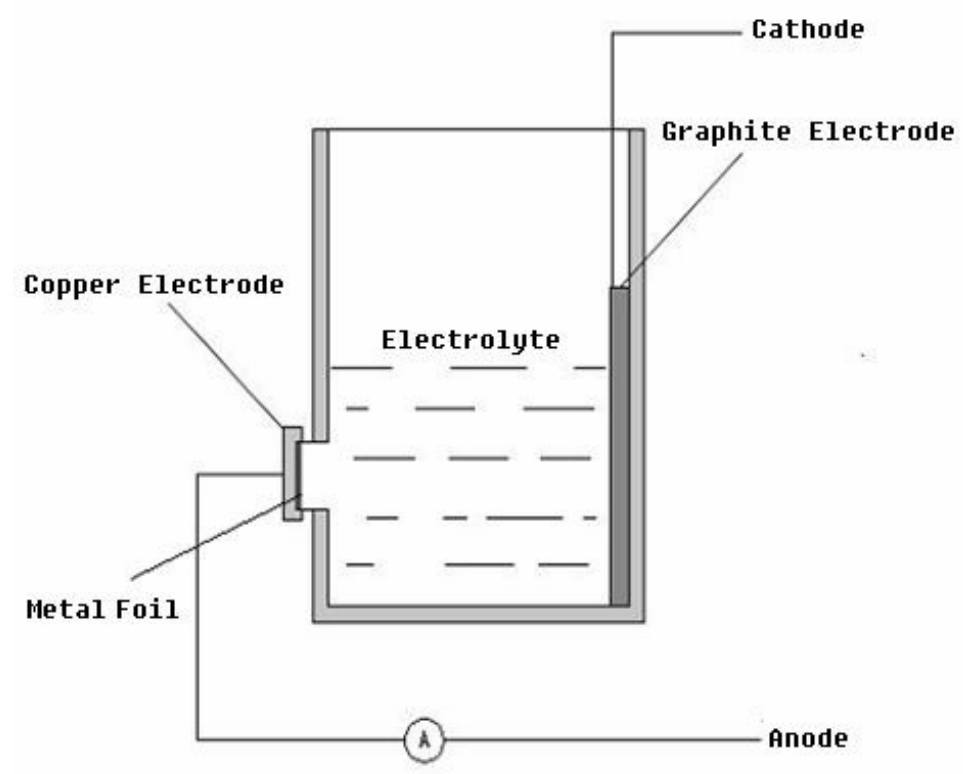

Figure 2-1. Schematic diagram of the apparatus used for the anodisation. 
When the circuit is closed, electrons are withdrawn from the metal at the positive terminal. With the presence of electric field, water dissociation at the metal surface near the anode was greatly enhanced. The dissociated oxygen anions were driven by the electric field to oxidize the metal base, forming the anodic metal oxide. Meanwhile, the anodic oxide produced dissolved in the electrolyte assisted by the electric field. Subsequently, porous anodic metal oxide grew at the equilibrium between oxidation and dissolution. The electrons withdrawn from the anode return to the bath at the cathode where they react with hydrogen ions to make hydrogen gas.

\subsubsection{Anodic aluminium oxide}

High purity aluminium foil $(0.1 \mathrm{~mm}, 99.99 \%)$ was sonicated in acetone for a few minutes and then rinsed in deionized water, without any other pre-treatments such as annealing and electropolishing. The anodisation was performed in a home-made cell with typical conditions: anodisation voltage of $40 \mathrm{~V}$ from Consort EV243 DC power supply, 0.3 $\mathrm{M} \mathrm{H}_{2} \mathrm{C}_{2} \mathrm{O}_{4}$ as electrolyte and a room temperature of $15{ }^{\circ} \mathrm{C}$. AAO specimens were collected with different anodisation times in order to investigate the pore formation at an early stage and how the pores developed with the anodisation time. To investigate the pore formation under different conditions, one specimen was prepared by dipping an aluminium foil into a $0.3 \mathrm{M}$ oxalic acid for $20 \mathrm{~h}$ without applying an electric field. Some anodisation experiments were performed at $120 \mathrm{~V}$ in $2 \%$ phosphoric acid. To examine the surface morphology of the aluminium substrate at the pore base, the oxide films were stripped off by a mixed solution of $0.2 \mathrm{M} \mathrm{H}_{2} \mathrm{CrO}_{4}$ and $0.4 \mathrm{M} \mathrm{H}_{3} \mathrm{PO}_{4}$.

Observation of the surface morphologies for all samples was carried out using scanning electron microscopy (SEM) on a JEOL JSM 5600 microscope and a FEI Sirion 200 field emission gun scanning microscope. To avoid charging, the samples were coated with gold before characterization. The SEM images were recorded with accelerating voltages from $5 \mathrm{KV}$ to 30 $\mathrm{KV}$.

\subsubsection{Anodic $\mathrm{TiO}_{2}$ nanotubes}

High purity titanium foil $(0.25 \mathrm{~mm}, 99.5 \%)$ was sonicated in acetone and then rinsed in 
deionized water. The anodisation was performed in a home-made cell with typical conditions: anodisation voltage range from DC $25 \mathrm{~V}$ to $60 \mathrm{~V}$, ethylene glycol containing $0.3 \mathrm{wt} \% \mathrm{NH}_{4} \mathrm{~F}$ and $2 \mathrm{wt} \%$ water as electrolyte, and room temperature of $15^{\circ} \mathrm{C}$. The anodising voltages were controlled by a high-voltage Consort EV243 DC power supply. Prepared $\mathrm{TiO}_{2}$ specimens were collected with different anodisation times from $20 \mathrm{~min}$ to $17 \mathrm{~h}$ in order to investigate the tube formation at different stages.

Observation of the morphology of the produced ATO films was carried out using SEM on a JEOL JSM-5600 microscope and a FEI Sirion 200 field emission gun scanning microscope. The working voltages were chosen as a compromise between high resolution and low charge effect with accelerating voltages ranging from $5 \mathrm{KV}$ to $30 \mathrm{KV}$. To gain profile information of the nanotubular layer, direct SEM cross-sectional measurements were carried out on mechanically bent and cracked samples. This technique was also applied to achieve bottom-view images of the nanotube layers. TEM and high resolution TEM (HRTEM) characterizations of individual $\mathrm{TiO}_{2}$ nanotubes were performed on JEOL JEM-2011 electron microscope operated at $200 \mathrm{kV}$, equipped with an Oxford Link ISIS energy dispersive X-ray spectroscopy (EDX) system and a Gatan 794 camera. Images were recorded at magnifications of 60,000 to $1,000,000 \times$. Crystallisation of the ATO films, by annealing from $285^{\circ} \mathrm{C}$ to $600^{\circ} \mathrm{C}$ in the air, was monitored by X-ray powder diffraction (XRD) on a Philips-1 diffractometer. Infrared spectra in a range of 400-4000 $\mathrm{cm}^{-1}$ were collected on a Perkin Elmer Spectrum GX IR spectrometer.

\subsection{Characterization methods}

The prepared AAO nanopores and the $\mathrm{TiO}_{2}$ nanotubes were analyzed using scanning electron microscopy (SEM), transmission electron microscopy (TEM), powder x-ray diffraction (XRD), and the infrared spectroscopy (IR).

\subsubsection{Electron microscopy}

Electron microscopes are scientific instruments that use beams of energetic electrons to examine objects on a very fine scale. Based on the design of optical microscopes, electron 
microscopes exploit the fact that fast moving electrons have a much smaller wavelength than visible light, which results in high-resolution images. Electron microscopes use an electron gun to generate the beam of energetic electrons. Whereas the light microscope uses glass lenses to magnify and focus images, an electron microscope uses magnetic lenses to magnify and focus images. Since electrons cannot travel freely in air, electron microscopes are built into airtight metal tubes or "columns" and use vacuum pumps to remove all the air within. There are two main types of electron microscope: (1) the scanning electron microscope (SEM) and (2) the transmission electron microscope (TEM). In general, the TEM image provides high-resolution information on the internal structure of a specimen while the SEM provides a detailed image of the surface structure of a sample. It is clear that both electron microscopes provide higher resolution images than is possible using a light microscope.

\subsubsection{Scanning electron microscopy}

The image is formed in an SEM by scanning an electron beam across a sample and collecting some signal from the beam-sample interaction, which is used to control the intensity of the spot on a television monitor which is scanning in synchronization with the beam on the sample. The nature of the signals collected by an SEM in order to form images is dependent on the detector used to collect them.

As shown in Figure 2-2, the SEM column consists of the electron gun and then several magnetic condense lenses, which are used to focus the electron beam into a small spot onto the sample surface. Unlike the TEM, there are no lenses after the specimen in a SEM. Scan coils are used to deflect the finely focused electron beam to create a tiny rectangular grid of parallel lines over the area of interest, while detectors measure the number of electrons that are displaced from each point on the surface. An image of the scanned area is generated on a video display, which is synchronized with the scan coils of the SEM so that the relative position of features is correctly displayed. The magnification of an SEM image is governed by the difference between the dimensions of the video display (normally fixed) and that of the area scanned on the specimen surface. Increasing magnification is achieved by scanning smaller and smaller areas on the specimen. 


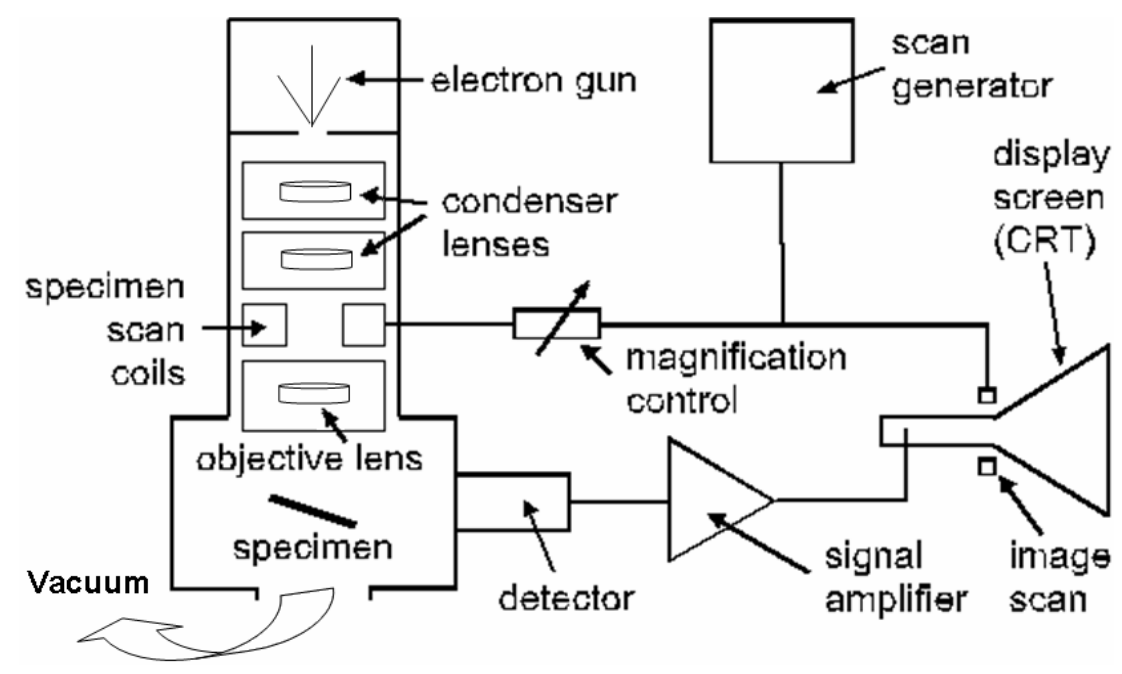

Figure 2-2. Schematic diagram for a SEM.

The scanning electron microscope allows the observation and characterization of heterogeneous organic and inorganic materials down to $\mathrm{nm}$ scale. As a result of the interaction of the electron beam with the sample, several types of signals are generated mainly including:

Secondary electrons (SEs): SEs are low energy electrons emitted from the sample due to the energetic passage of beam electrons, with typical energies of the order of a few eV. SEs are emitted throughout the interaction volume, but they don't travel far in the sample before being absorbed. That is why SEs are considered as a surface-sensitive signal. Back Scattered Electrons (BSEs): BSEs are primary beam electrons that have undergone elastic collisions within the sample and are detected from the surface they came in. X-rays: characteristic X-rays are produced when a beam electron knocks out an inner shell electron, producing a 'hole'. This hole is quickly filled by an electron from a higher energy level within the same atom. The energy difference between the electrons facilitates the release of electromagnetic energy by emitting x-rays. Thus, the energies of the characteristic x-rays emitted are dependent on the energy level differences between the electron shells in an atom, which are different from different elements.

\section{Detectors}

There are two main types of electron detectors used when obtaining a SEM image. The first measures the number of backscattered electrons. The second detector measures the number of secondary electrons. Images generated using secondary electrons are extremely sensitive to 
surface morphology, while those generated using backscattered electrons are sensitive to the atomic weight. It is relatively easy to make stereo pairs of images by tilting the specimen a few degrees along an axis parallel to the viewing direction. These often give a much better impression of the surface relief than single pictures.

The EDS detector is usually an ideal intrinsic semiconductor. This intrinsic semiconductor is cooled typically by liquid nitrogen, so there is very little thermionic creation of charge carriers. The potential drop between the front and back contacts of the detector is kept at several kilovolts. $\mathrm{X}$-rays that pass through the front contact will tend to lose their energy creating electron-hole pairs in the intrinsic region; because each electron-hole pair has a characteristic creation energy, the total number of charge carriers created is proportional to the energy of the incident $\mathrm{x}$-ray. Thus, by measuring the charge pulse that is created for each $x$-ray, the energy of the x-ray can be determined. A computer keeps track of the number of counts within each energy range, and the total collected x-ray spectrum can then be determined.

\section{Specimen preparation}

A major advantage of the SEM (in comparison to a TEM) is the ease of specimen preparation. In fact, many conducting specimens require no special preparation before use in the SEM. However, poorly conducting specimens tend to charge up electrostatically when under illumination of the scanning electron probe, since they provide no path to ground for the specimen current $\mathrm{I}_{\mathrm{S}}$. This current could be positive or negative, depending on the backscattering coefficient and secondary-electron yield. Negative charge is the more serious problem, since it will deflect the primary-electron beam, resulting in image distortion or fluctuations in image intensity. Conservation of charge implies that the electron current $\mathrm{I}_{\mathrm{S}}$ which flows to ground is equal to the primary-beam current $\mathrm{I}_{\mathrm{P}}$ minus the rate of loss of electrons from secondary emission and backscattering:

$\mathrm{I}_{\mathrm{S}}=\mathrm{I}_{\mathrm{P}}-\mathrm{I}_{\mathrm{BSE}}-\mathrm{I}_{\mathrm{SE}}=\mathrm{I}_{\mathrm{P}}(1-h-d)$

One solution to the problem of charging is to coat the surface of the SEM specimen with a thin film of a gold or graphitic carbon. Films of 10-50 nm thickness conduct sufficiently well to 
prevent charging at the surface. Since this thickness is greater than the SE escape depth, secondaries are recorded from the coating rather than from the original specimen. However, as the external contour of such a thin film closely follows that of the specimen, image obtained could be rather faithful.

Where coating is impossible or difficult, specimen charging can often be avoided by choice of SEM accelerating voltages. The values of backscattering coefficient $h$ and secondary-electron yield $d$ for a given material depend on the primary-electron energy $E_{0}$. For high $E_{0}$, the penetration depth is large and only a small fraction of the excited atomic electrons escape into the vacuum as secondaries. Also, BSEs which are generated deep within the specimen will not have enough energy to escape, so $h$ will be low. Low total yield of $(h+d)$ means that the specimen will charge negatively. As $E_{0}$ is reduced, $d$ increases and the specimen current required to maintain charge neutrality eventually falls to zero at some incident energy $E_{2}$, where $(h+d)$ $=1$. Further reduction in $E_{0}$ could result in a positive charge, but this would attract secondaries back to the specimen, thereby neutralizing the charge, so it is not usually a problem.

For $E_{0}$ less than some energy $E_{1}$, the primary electrons do not have enough energy to create secondaries, $d=0$. Considering that $h<1$, negative charging occurs again. As shown in Figure $2-3$, there is a range of primary energy $\left(E_{1}\right.$ to $\left.E_{2}\right)$ over which negative charging does not occur, even for an insulating specimen. Usually $E_{2}$ is found experimentally, by reducing the voltage until charging effects in the image disappear.

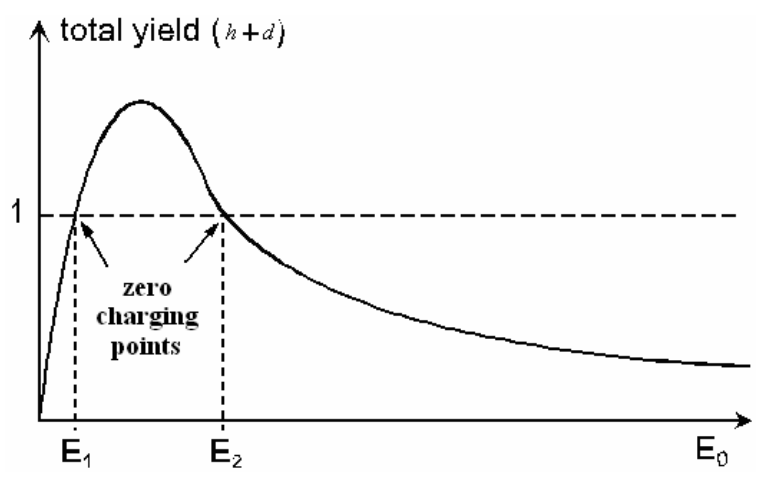

Figure 2-3. Total yield $(h+d)$ as a function of primary-electron energy, showing the range $\left(E_{l}\right.$ to $E_{2}$ ) over which no negative specimen charging occurs. 
Therefore, the low-voltage SEM could be an attractive option for imaging insulating specimens. However, there is a downside to low $E_{0}$ : increased chromatic-aberration broadening of the electron probe hence decreasing the resolution. This problem can be minimized by using a field-emission source whose objective lenses has a low chromatic-aberration coefficient, and it has a low energy spread also: $\Delta E<0.5 \mathrm{eV}$.

\section{Field-emission scanning electron microscope}

In standard electron microscopes electrons are mostly generated by heating a tungsten filament by means of a current to a temperature of about $2800^{\circ} \mathrm{C}$ (electron gun). Sometimes electrons are produced by a crystal of lantanumhexaboride $\left(\mathrm{LaB}_{6}\right)$ that is mounted on a tungsten filament. This modification results in a higher electron density in the beam and a better resolution. In a field emission (FE) scanning electron microscope no heating but a so-called "cold" source is employed. An extremely thin and sharp tungsten needle (tip diameter $10^{-7}-10^{-8} \mathrm{~m}$ ) functions as a cathode in front of a primary and secondary anode. In contrast to a conventional tungsten filament, a FE tip last theoretically for a lifetime.

Because the electron beam produced by the FE source is about 1000 times smaller than in a standard microscope, the image resolution is dramatically better. High quality images could be obtained with negligible electrical charging of samples at low accelerating voltage, and the need for placing conducting coatings on insulating materials is virtually eliminated.

In the present work, SEM characterizations were carried out using a JEOL JSM 5600 fitted with a tungsten filament as the electron source or a FEI Sirion 200 field emission gun scanning microscope. To avoid the electrical charging effect, a covering of thin gold film was deposited onto the surface of the AAO before SEM characterization. While during the characterization of ATO, the working voltage is normally limited to $15 \mathrm{KV}$. In both cases, completely dry specimens are required. Some AAO and ATO samples were cut mechanically for the requirement of sectional observation.

\subsubsection{Transmission electron microscopy}

HRTEM instrument is composed of electron-optical system, vacuum system and electrical system. The electron-optical system includes an electric illumination system, imaging system, 
image observation and recording system, and sample chamber. The electric illumination system can be divided into the electron gun, condenser lens and adjustment device. The electron gun can use three kinds of electron sources, a plain tungsten (W) filament, a W filament with a $\mathrm{LaB}_{6}$ crystal tip or a field emission gun. The acceleration voltage is usually $50 \sim 200 \mathrm{kV}$. The condenser lens is used to converge the electron beam emitted from the electron gun to penetrate samples. The imaging system of a TEM is usually composed of an objective lens, intermediate lens and a projector lens. The high and low magnification imaging can be adjusted by using all these lens or just some of them.

The electron will lose energy by collision with gas in air, which causes the decrease of the average free path of electron motion. Thus a vacuum, at least $1.33 \times 10^{-2} \mathrm{pa}$, is necessary for the electron to pass completely down the column $\left(1.33 \times 10^{-4}\right.$ pa $\sim 1.33 \times 10^{-5}$ pa is for $200 \mathrm{kV}$ acceleration voltage). Additionally, the high vacuum can prolong the filament life, keep good insulation surroundings and reduce the sample contamination.

The electrical system includes a high voltage power supply for the electron gun, current power supply for the magnetic lens, power supply of various electrical equipment, power supply of vacuum system, safety protection electrical equipment and computer control system.

The basic layout of a TEM is shown in Figure 2-4. The electron beam for TEM is generated in an electron gun by heating the $\mathrm{LaB}_{6}$ filament in vacuum. The electrons are accelerated from the gun (cathode) towards the anode by applying a high voltage (200 KV). The electrons will have no potential energy and high kinetic energy as they arrive at anode. The accelerated beam is focused by a set of condenser lens, on the specimen. After the beam passes through a specimen, the objective lens produces the primary magnified image of the specimen. The specimen image was further enlarged many times by a series of projector lenses. Finally the beam electrons are transferred to the resulting image displayed on the viewing screen or recorded photographically or digitally (Figure 2-4). 


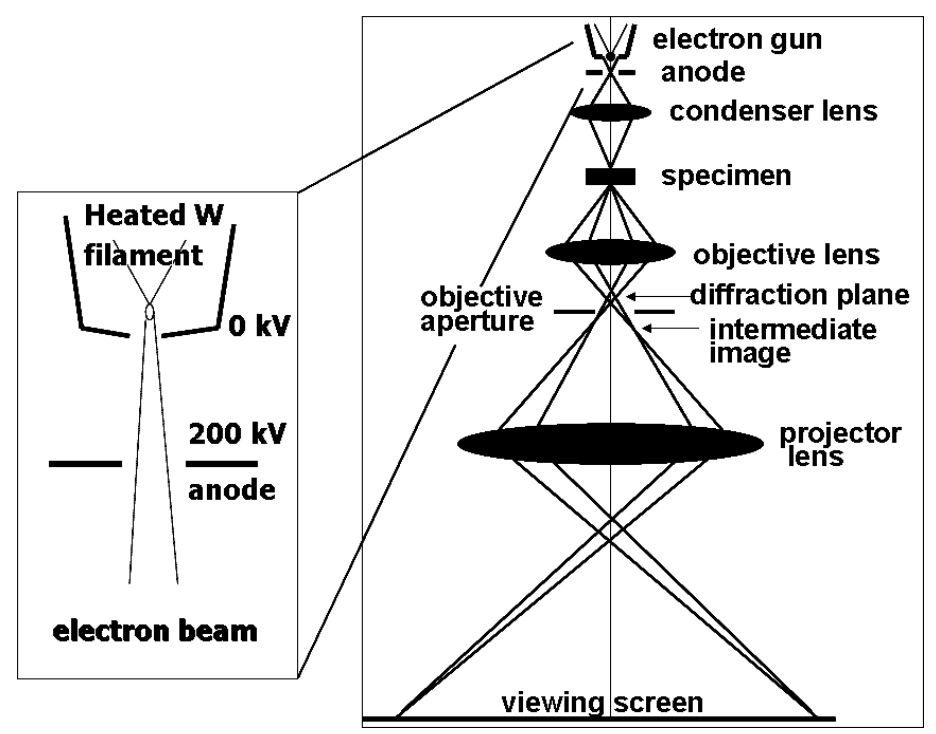

Figure 2-4. Schematic diagram for TEM.

\section{Electron beam as a light source}

As an ideal point light source is imaged by a lens, there is a bright region in the centre (airy disk) with a series of concentric rings in the image plane, which is called airy pattern. One airy disk will be overlapped by another when the distance between two objective points decrease, which causes low resolution and limits the magnification of microscopy. The lowest resolution distance was determined as the distance between two objective points $\left(\Delta r_{0}\right)$ when the distance between two corresponding airy disk centers is equal to one airy disk's radius. The value of $\Delta r_{0}$ is positively correlated with the wavelength $(\lambda)$ of light source. Thus a high resolution can be obtained by using an electron beam with a small wavelength, i.e., an electron beam with high accelerating voltage.

Transmission electron microscopy uses an electron beam as a light source. In 1924, De Broglie proved that a particle with high speed can be treated as electromagnetic radiation with a wavelength $\lambda=\frac{h}{m v}$, where $h$ is Planck's constant. The wavelength $(\lambda)$ is equal to Planck's constant $(h)$ divided by the product of the mass $(m)$ and the velocity $(v)$ of the particle. In TEM, the velocity of an electron is relative to acceleration voltage $e V=\frac{1}{2} m v^{2}$. The kinetic energy of an electron $\left(1 / 2 \mathrm{~m} v^{2}\right)$ is equal to the multiple of electron charge $(e)$ and the accelerating voltage $(V)$. As the acceleration voltage is very high, the velocity of the electron will be comparable to 
the speed of light and the relativistic corrections should be considered, therefore,

$\lambda=\frac{h}{\sqrt{2 e m_{0} V\left(1+\frac{e V}{2 m_{0} c^{2}}\right)}}\left(m_{0}\right.$ is the rest mass of electron $)$

$\lambda$ obtained by this equation (2.2) can be expressed as $\lambda=\frac{12.25}{\sqrt{V\left(1+0.9788 \times 10^{-6} V\right)}}$. The value of the wavelength is $0.00251 \mathrm{~nm}$ when the voltage is $200 \mathrm{kV}$, which is much smaller than that of visible light $(390 \sim 760 \mathrm{~nm})$ and X-rays (e.g. $\lambda$ of $\left.\mathrm{Cu} \mathrm{K}_{\alpha}=0.154 \mathrm{~nm}\right)$.

\section{Image contrast of TEM}

The image contrast of TEM, including scattering (mass-thickness) contrast, diffraction contrast and phase contrast, is the foundation of TEM images. Differences in mass and thickness in the parts of samples give different scattering ability, which generates scattering contrast. The intensity of the electron beam $(I)$ can be calculated by using the thickness $(t)$, atomic weight $(A)$ and density $(\rho)$ of the sample and scattering cross section $\left(\sigma_{\alpha}\right): I=I_{0} e^{-\frac{k \sigma_{\alpha}}{A} \cdot \rho t}$. The different intensity of the electron beam generates the scattering contrast image of samples.

Phase contrast comes from phase differences generated when electrons passing through the sample. The scattered wave and transmitted wave lead to the interference in the image. Various phase differences lead to a differential interference on the image plane, which makes a phase contrast image.

Some samples have uniform thickness and near-neighbor atomic number and the scattering contrast in this sample image is not obvious. The TEM image contrast of this thin sample is relative to the crystal structure of this sample. Diffraction contrast is caused by addition of the diffracted beam contrast, which is relative to the crystal structure of the sample, to transmitted beam intensity. Accordingly, the diffraction contrast image reflects the crystal characterization of samples.

In the present work, high resolution transmission electron microscopy (HRTEM) was carried out on a JEOL JEM 2011 electron microscope fitted with a $\mathrm{LaB}_{6}$ filament, operating at $200 \mathrm{kV}$. 
The resolution can be up to $0.18 \mathrm{~nm}$ and the useful magnification is up to $1,200,000 \mathrm{x}$. TEM images were recorded using a Gatan CCD camera. EDX experiments were carried out using an Oxford Instruments X-ray Analysis ISIS 300 system with a silicon detector and beryllium window attached to the microscope. EDX was used to qualitatively determine the elements present in a sample. Selected area electron diffraction (SAED) was carried out using a double tilt sample holder with $\pm 20^{\circ}$ tilting capability to determine the crystallinity of the material and to identify the crystallographic system.

The important factors influencing the quality of TEM image resolution are various types of aberration. It is impossible to get a $100 \%$ perfect magnetic lens, so the defects of magnetic lens lead to spherical aberration, astigmatism, image distortion and chromatic aberration. The spherical aberration results from the different refraction ability of electron beam between magnetic fields in adaxial area and abaxial area, and leads to a least defocus spot $\left(r_{s}\right)$. The radius of the least defocus spot can be calculated by equation (2.3) with the spherical-aberration coefficient $\left(C_{s}\right)$ and the half-aperture angle $(\alpha)$ of electromagnetic lens.

$$
r_{s}=\frac{1}{4} C_{s} \alpha^{3}
$$

According to the equation (2.3), when the spherical-aberration coefficient $\left(C_{s}\right)$ or the half-aperture angle $(\alpha)$ of electromagnetic lens are reduced, the least defocus spot $\left(r_{s}\right)$ decreases, which accordingly reduces the influence of spherical aberration and increases the quality of the image resolution. Therefore, the increase of the magnetic field intensity and the decrease of the grating aperture are selected to reduce the spherical-aberration coefficients $\left(C_{s}\right)$ and can reduce the half-aperture angle $(\alpha)$, respectively.

The change of the wavelength or energy leads to the focus change of electromagnetic lens and chromatic aberration. The least defocus spot $\left(r_{c}\right)$ caused by chromatic aberration can be obtained by equation (2.4) ( $\Delta E / E$ is the energy rate of the change of electron beam).

$$
r_{c}=C_{c} \alpha\left|\frac{\Delta E}{E}\right|
$$


The method to reduce the chromatic aberration is via improving the stability of acceleration voltage, increasing the magnetic field intensity and reducing the chromatic aberration coefficients $\left(C_{c}\right)$.

Astigmatism is a kind of aberration caused by non-rotational symmetry of the magnetic field of the electromagnetic lens. The least defocus spot $\left(r_{a}\right)$ can be calculated by equation $(2.5)\left(\Delta f_{a}\right.$ is the focus difference caused by non-rotational symmetry of magnetic field).

$$
r_{a}=\frac{\Delta f_{a} \alpha}{2}
$$

Astigmatism can be emended by using anastigmatic instrument.
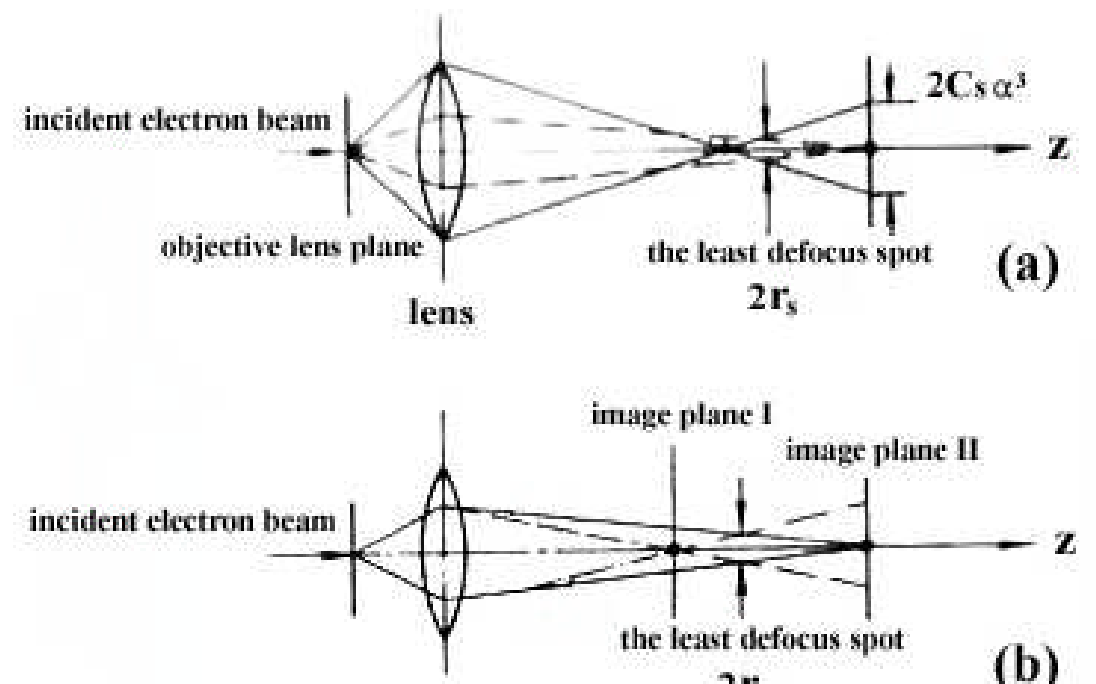

$2 r_{\mathrm{c}}$

(b)

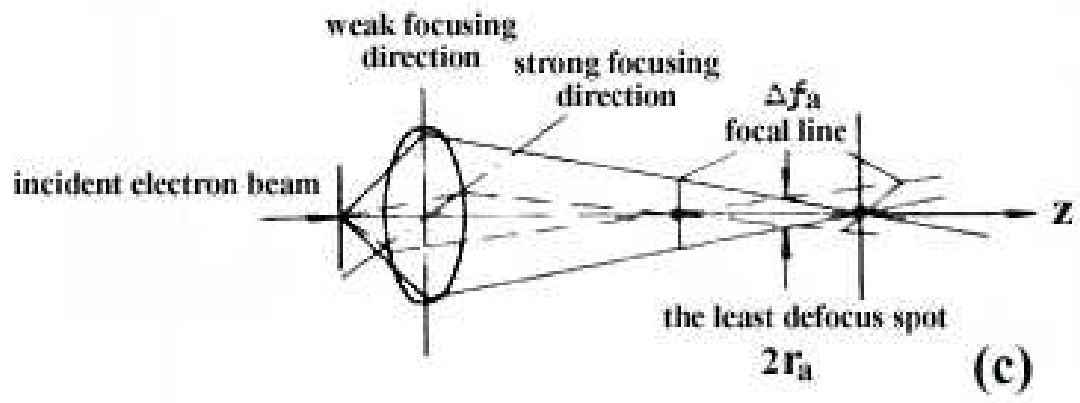

Figure 2-5. Schematic drawing of the aberration formation in TEM. (a) spherical aberration, (b) chromatic aberration and (c) astigmatism.

Figure 2-5 shows the fundamental principle of these three types of aberration formation in 
TEM. Additionally, a kind of diffraction aberration results from the diffraction effect in TEM and can be reduced by increasing the half-aperture angle $(\alpha)$ of the electromagnetic lens, which also increases spherical aberration. Therefore a proper half-aperture angle $(\alpha)$ is selected by taking the two aberrations into consideration.

\section{TEM specimen preparation}

Two factors complicate the imaging of samples in a TEM. First, due to the limited penetration of electrons in matter, specimens for TEM must be extremely thin (approximately 0.1 micron or less). Second, since the specimens must be inserted into the electron microscope column which is under vacuum, they must be dry.

There is a range of TEM specimen preparation techniques available depending on the type of sample. Solid, inorganic samples can be thinned using a combination of mechanical, chemical, and ion beam thinning. As with most kinds of microscopy, specimen preparation is an important and specialized area of expertise.

In the present work, the ATO samples were ground well using a pestle and mortar, then acetone was added to the sample and the suspension was ground again. One drop of the suspension was deposited on a holey carbon film on a $3 \mathrm{~mm}$ copper grid. The sample was allowed to dry in air fully prior to examination in the TEM.

\section{Selected area electron diffraction}

Selected area electron diffraction (SAED) is a technique used in TEM to examine the crystallography of a sample. In a TEM, the ordered atoms in a specimen act as a diffraction grating to the electrons because the wavelength of high-energy electrons is much smaller than the spacings between neighboring atoms in a specimen. Thus the electron beam passes through a thin crystalline specimen and is scattered to form diffracted beams with some particular angles determined by the crystal structure of the sample. There are three basic SAED patterns according to different crystallinities of samples. A regular $2 \mathrm{D}$ pattern indicates that the specimen is a single crystal (Figure 2-6a), a set of dotted rings indicate polycrystalline material (Figure 2-6b) and a set of diffuse rings indicate amorphous material (Figure 2-6c). The specimen often needs to be tilted to a particular angle in both $\mathrm{x}$ and $\mathrm{y}$ directions to obtain the best diffraction patterns. 


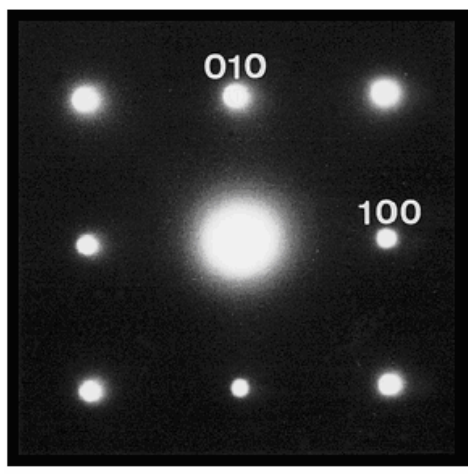

(a)

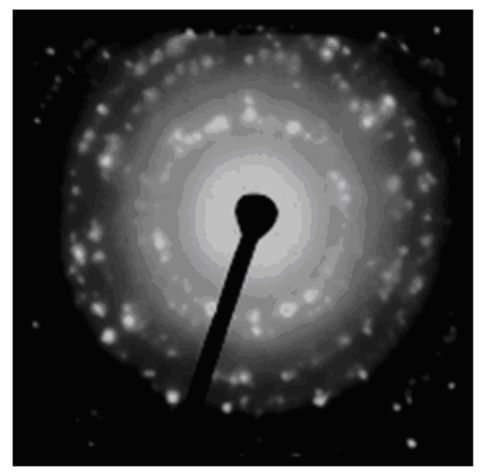

(b)

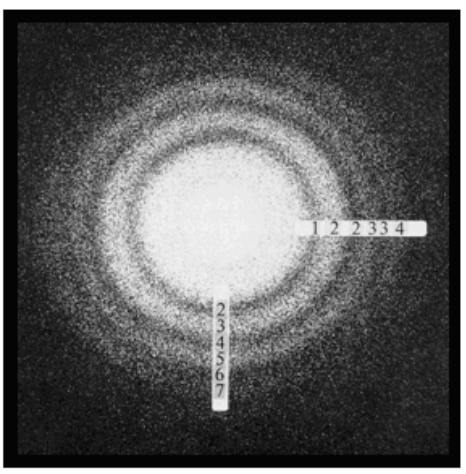

(c)

Figure 2-6. SAED patterns of (a) single crystal, (b) polycrystalline particle and (c) amorphous particle.

Figure 2-7 shows the diagram of the relationship between the reciprocal d-spacing $\left(d^{*}\right)$ and the distance between the diffraction rings or spots $\left(D^{*}\right)$. The d-spacing (d) of crystalline specimen can be calculated by equation (2.6) where $\mathrm{d}^{*}$ is the reciprocal d-spacing, $\mathrm{D}^{*}$ is the distance between the central spot and a diffraction spot on the negative and $\mathrm{L}$ is the camera length.

$d=\frac{1}{d^{*}}=\frac{\lambda}{2 \sin \theta}=\frac{L \lambda}{D^{*}}$

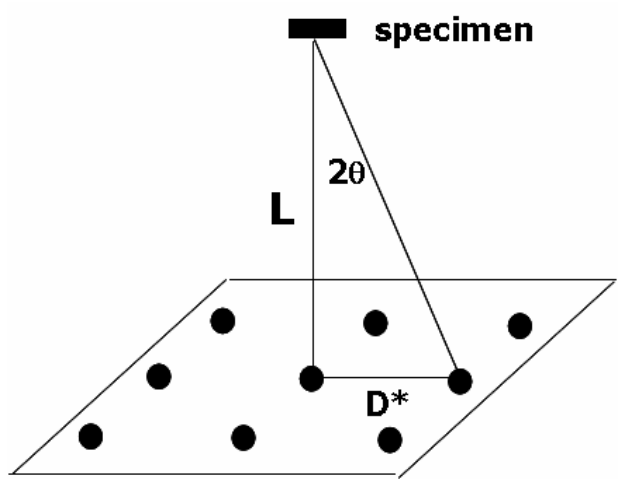

Figure 2-7. Diagram of relationship between $d^{*}$ and $D^{*}, D^{*}$ is a distance between the central spot and a diffraction spot and $\mathrm{L}$ is camera length.

An SAED pattern is a magnified image of the reciprocal lattice cross-section and can reflect directly to the crystal structure of specimens. If the unit cell of a crystalline sample is known, 
SAED patterns can be easily indexed. If the structure is unknown, two or more diffraction patterns from different directions are required to reveal the unit cell.

\section{Energy dispersive x-ray analysis}

Energy dispersive $\mathrm{x}$-ray spectroscopy (EDX) is an analytical technique used for elemental analysis and determination of chemical composition of a material. Each element has a unique atomic structure and emits characteristic x-rays when it is hit by an incident electron beam. In EDX, a high energy electron beam excites and ejects an electron in an inner shell of an atom. An electron from an outer shell with a higher energy fills the electron hole where the emitted electron was and the difference in energy between the energy shells is released in the form of $\mathrm{X}$-rays. The wavelength or energy of this kind of $\mathrm{x}$-ray is unique for different elements. Therefore the elements present in a specimen can be determined by EDX.

Moreover, the intensity of the characteristic x-ray is relative to the elemental concentration of the specimen so that the proportion of all kinds of elements in the samples can be calculated using EDX. The local defects in the sample can be detected by using elemental mapping, which gives the distribution of the target element.

\subsubsection{X-ray diffraction}

X-ray diffraction (XRD) is a versatile, non-destructive technique that reveals detailed information about the chemical composition and crystallographic structure of natural and manufactured materials. XRD data was collected from a large amount of sample, which transfers $3 \mathrm{D}$ information to a $1 \mathrm{D}$ display. It is difficult to index the peaks or work out the structure if the sample is unknown. But if a sample is limited to a range of known structures and compositions, XRD is a fast way to determine the structure of this sample.

\subsubsection{Lattice planes and Bragg's law}

$\mathrm{X}$-rays primarily interact with electrons in atoms. When $\mathrm{x}$-ray photons collide with electrons, some photons from the incident beam will be deflected away from the direction where they originally travelled. If the wavelength of these scattered x-rays did not change (meaning that 
$\mathrm{x}$-ray photons did not lose any energy), the process is called elastic scattering in which only momentum has been transferred in the scattering process. These are the $\mathrm{x}$-rays that we measure in diffraction experiments, as the scattered $\mathrm{x}$-rays carry information about the electron distribution in materials. On the other hand, in the inelastic scattering process, $\mathrm{x}$-rays transfer some of their energy to the electrons in specimen and the scattered x-rays will have different wavelength than the incident $\mathrm{X}$-rays.

Diffracted waves from different atoms can interfere with each other and the resultant intensity distribution is strongly modulated by this interaction. If the atoms are arranged in a periodic fashion, as in crystals, the diffracted waves will consist of sharp interference maxima (peaks) with the same symmetry as in the distribution of atoms. Analysing diffraction patterns therefore allows us to deduce the distribution of atoms in crystals.

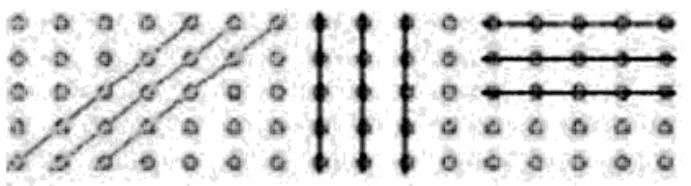

Lattice Planes

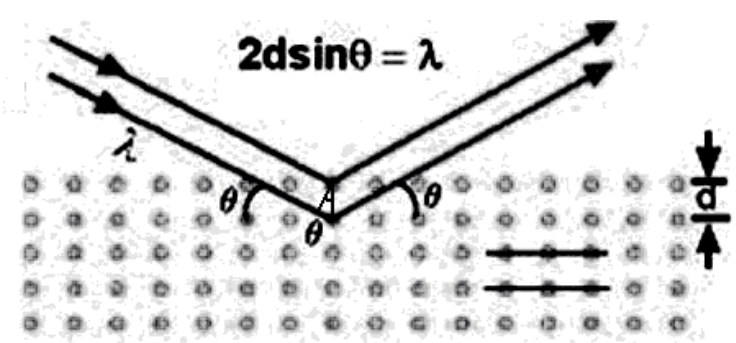

Bragg's Law

Figure 2-8. Schematics for the lattice planes and the Bragg's scattering law.

The peak positions in an x-ray diffraction pattern are directly related to the atomic distances. Let us consider an incident x-ray beam interacting with periodically arranged atoms as shown in Figure 2-8. The atoms, represented as gray spheres in the schematic, can be viewed as forming different sets of planes in the crystal (lines in schematic on the left). For a given set of lattice planes with an inter-plane distance of $d$, the condition for a diffraction (peak) to occur can be simply written as $2 d \sin \theta=\mathrm{n} \lambda$. which is known as the Bragg's law. In the equation, $\lambda$ is the wavelength of the $\mathrm{x}$-ray, $\theta$ the scattering angle, and $\mathrm{n}$ an integer representing the order of the diffraction peak. The Bragg's Law is one of most important laws used for interpreting x-ray diffraction data. It is important to point out that although we have used atoms as scattering points in this example, Bragg's Law applies to scattering centers consisting of any periodic 
distribution of electron density. In other words, the law holds true if the atoms are replaced by molecules or collections of molecules, such as colloids, polymers, proteins and virus particles.

\subsubsection{Powder XRD}

Powder XRD is perhaps the most widely used x-ray diffraction technique for characterizing materials. As the name suggests, the sample is usually in a powder form, consisting of fine grains of crystalline material to be studied. The technique is also widely used for studying particles in liquid suspensions or polycrystalline solids (bulk or thin film materials). The term 'powder' really means that the crystalline domains are randomly oriented in the sample. Therefore when the 2-D diffraction pattern is recorded, it shows concentric rings of scattering peaks corresponding to the various d spacings in the crystal lattice. The positions and the intensities of the peaks are used for identifying the underlying structure (or phase) of the material.

Powder diffraction data can be collected using either transmission or reflection geometry, as shown below in Figure 2-9. Because the particles in the powder sample are randomly oriented, these two methods will yield the same data.

\section{Reflection mode}

Reflectometry is an analytical technique for investigating thin layers using the effect of total external reflection of x-rays. In reflectivity experiments, $\mathrm{x}$-ray reflection of a sample is measured around the critical angle. Below the critical angle of total external reflection, x-rays penetrate only a few nanometers into the sample. Above this angle the penetration depth increases rapidly. At every interface where the electron density changes, a part of the x-ray beam is reflected. The interference of these partially reflected x-ray beams creates an oscillation pattern observed in reflectivity experiments. From these reflectivity curves, layer parameters such as thickness and density, interface and surface roughness can be determined. Reflectometry is applied to characterize single and multi-layer structures and coatings from among many others, magnetic, semiconducting and optical materials. To increase the angle of incident $\mathrm{x}$-ray beam, powder XRD carried out in reflection mode could be used to study the internal crystal structure of the sample also. 


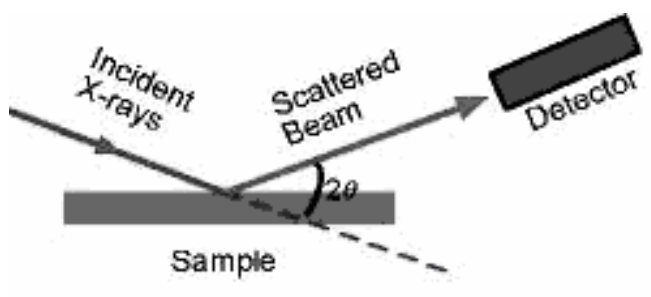

Reflection

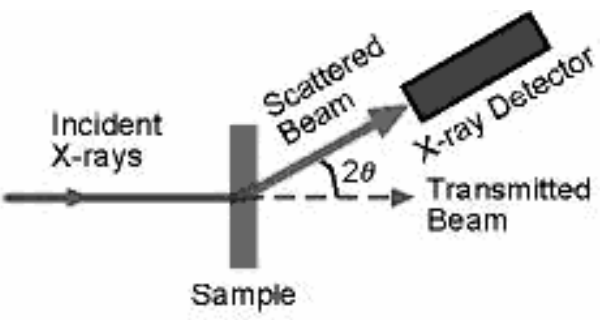

Transmission

Figure 2-9. Reflection and transmission modes of powder XRD.

\section{Transmission mode}

In transmission $\mathrm{x}$-ray diffraction experiments, the incident $\mathrm{x}$-ray beam is not reflected by the sample, but instead travels through the sample, where the diffraction process takes place. In the transmission geometry the x-ray beam is focused on the detector by an incident beam conditioner - an x-ray mirror, for instance. The foil sample is placed perpendicular to the diffraction plane in the incident beam. The x-ray beam is diffracted over the Bragg angle and focused on the detector on the $2 \theta$ circle.

Additionally, crystallite size ( $\mathrm{D}$, grain size) can also be calculated via the characteristic peak width of an XRD pattern by using the Debye-Scherrer formula (2.7), where $\beta$ is the width of the peak at half maximum intensity of a specific plane (hkl), $k$ is a constant that varies with the method of taking the breadth $(0.89<\mathrm{k}<1), \lambda$ is the wavelength of incident $\mathrm{x}$-rays and $\theta$ is the centre angle of the peak. It is the quantitative equivalent of saying that the XRD peaks are wider when the sample's crystallites are smaller.

$$
D_{h k l}=\frac{k \lambda}{\beta \cos \theta}
$$

In the present work, ATO samples were measured by a Philips diffractometer operating in reflection mode. Powder x-ray diffraction patterns were recorded using the $\mathrm{CuK}_{\alpha 1}$ radiation $(1.54 \AA)$ as the $\mathrm{x}$-ray source. To study their internal crystal structure, data was collected between $2 \theta=15^{\circ}$ and $100^{\circ}$. A typical scan rate of $1^{\circ} \mathrm{min}^{-1}$ and step size of $0.5^{\circ}$ were used. The interplanar d-spacings corresponding to each peak were calculated from their respective $2 \theta$ values using 
Bragg equation: $d=\frac{n \lambda}{2 \sin \theta}$, Where $\mathrm{n}$ is the order of diffraction and $\lambda$ is the $\mathrm{Cu}-\mathrm{K}_{\alpha}$ wavelength.

\subsubsection{Infrared spectroscopy}

An infrared (IR) spectrum represents a fingerprint of a sample with absorption peaks which correspond to the frequencies of vibrations between the bonds of the atoms. Because each different material is a unique combination of atoms, no two compounds produce the same IR spectrum. Therefore, IR spectroscopy can give a good identification (qualitative analysis) of different materials. In addition, the size of the peaks in the spectrum is a direct indication of the amount of material present.

Because each interatomic bond may vibrate in different motions (stretching or bending), individual bonds may absorb at more than one IR frequency. Stretching absorptions usually produce stronger peaks than bending, however the weaker bending absorptions can be useful in differentiating similar types of bonds. It is also important to note that symmetrical vibrations do not cause absorption of IR radiation.

In the present work, in order to confirm the existence of titanium hydroxide in as-prepared ATO samples, infrared spectra in a range of $400-4000 \mathrm{~cm}^{-1}$ were collected on a Perkin Elmer Spectrum GX IR spectrometer with the help of Mrs Sylvia Williamson.

\subsubsection{Computational chemistry}

Computational chemistry is a branch of chemistry that uses computers to assist in solving chemical problems. It combines the theoretical chemistry and efficient computer programs, to calculate the structures and properties of molecules and solids. Computational chemistry is usually used when a mathematical method is sufficiently well developed that it can be automated for implementation on a computer. Though very few aspects of chemistry can be computed exactly, almost every aspect of chemistry can be described in a qualitative or approximate quantitative computational scheme. Computational studies can be carried out in order to find a starting point for a laboratory synthesis, or to assist in understanding experimental data. Also it can be used to predict the possibility of so far entirely unknown molecules or to explore reaction 
mechanisms that are not readily studied by experimental means.

With developed algorithms and computer programs to predict atomic and molecular properties and reaction paths for chemical reactions, computational chemists only need to apply existing computer programs and methodologies to specific chemical questions.

\subsubsection{Ab initio methods}

The programs used in computational chemistry are based on many different quantum-chemical methods that solve the molecular Schrödinger equation associated with the molecular Hamiltonian. Methods that do not include any empirical or semi-empirical parameters in their equations - being derived directly from theoretical principles, with no inclusion of experimental data - are called ab initio methods. This does not imply that the solution is an exact one; they are all approximate quantum mechanical calculations. It means that a particular approximation is rigorously defined on first principles (quantum theory) and then solved within an error margin that is qualitatively known beforehand. If numerical iterative methods have to be employed, the aim is to iterate until full machine accuracy is obtained.

The simplest type of ab initio electronic structure calculation is the Hartree-Fock scheme. This is an extension of molecular orbital theory, in which the correlated electron-electron repulsion is not specifically taken into account but only its average effect included. Many types of calculations (known as post-Hartree-Fock methods) begin with a Hartree-Fock calculation and subsequently correct for electron-electron repulsion. As these methods are pushed to the limit, they approach the exact solution of the non-relativistic Schrödinger equation. However, in order to obtain exact agreement with experiment, it is still necessary to include relativistic and spin orbit terms, both of which are only really important for heavy atoms.

\subsubsection{Density functional theory (DFT) methods}

Density functional theory (DFT) methods are often considered to be $a b$ initio methods for determining the molecular electronic structure, even though many of the most common functionals use parameters derived from empirical data, or from more complex calculations. In DFT, the total energy is expressed in terms of the total one-electron density rather than the wave function. In this type of calculation, there is an approximate Hamiltonian and an approximate 
expression for the total electron density. DFT methods can be very accurate for little computational cost. Some methods combine the density functional exchange functional with the Hartree-Fock exchange term and are known as hybrid functional methods.

\subsubsection{Polarizable continuum model (PCM)}

The Polarizable Continuum Model (PCM) is one of the most frequently used continuum solvation methods in computation chemistry. The PCM model calculates the molecular free energy in solution as the sum over three terms: $G_{s o l}=G_{e s}+G_{d r}+G_{c a v}$. These components represent the electrostatic (es) and the dispersion-repulsion (dr) contributions to the free energy, and the cavitation energy (cav). All three terms are calculated using a cavity defined through interlocking van der Waals-spheres centered at atomic positions. The reaction field is represented through point charges located on the surface of the molecular cavity.

In the present work, the computation was carried out using a Density functional theory (DFT) method at the B3LYP/6-311+G** level. A polarizable continuum model (PCM) was employed in the single-point energy computations. All computations were performed using the Gaussian03 program package by Prof. M. Bühl from the School of Chemistry in the University of St Andrews. 


\section{Formation of Self-ordered AAO Nanopore Arrays}

Porous anodic aluminium oxide (AAO) was first reported 50 years ago, ${ }^{24}$ and the material has attracted increasing attention from scientists in the fields of materials science, electrochemistry, nanomaterials and nanotechnology in recent years. ${ }^{217-220}$ However, the driving force for the self-organisation of the pores still remains unclear, although extensive research in the past years has made great achievements in this field. $27,40,75,76,89,94,221-224$

Figure 3-1 shows the morphologies of AAO samples prepared in $0.3 \mathrm{M}$ oxalic acid under $60 \mathrm{~V}$ and $40 \mathrm{~V}$. It can be seen that, the AAO film prepared under $40 \mathrm{~V}$ has a better ordered pore array, showing a hexagonal close-packed structure. While from the sectional view SEM images for AAO samples prepared in $2 \mathrm{wt} \%$ phosphoric acid under $120 \mathrm{~V}$ and $0.3 \mathrm{M}$ oxalic acid under $40 \mathrm{~V}$ (Figure 3-2), hemispherical pore bottoms with constant barrier layer thickness can be seen clearly. The pore arrays for AAO film prepared in $0.3 \mathrm{M}$ oxalic acid under $40 \mathrm{~V}$ show much better ordering again.
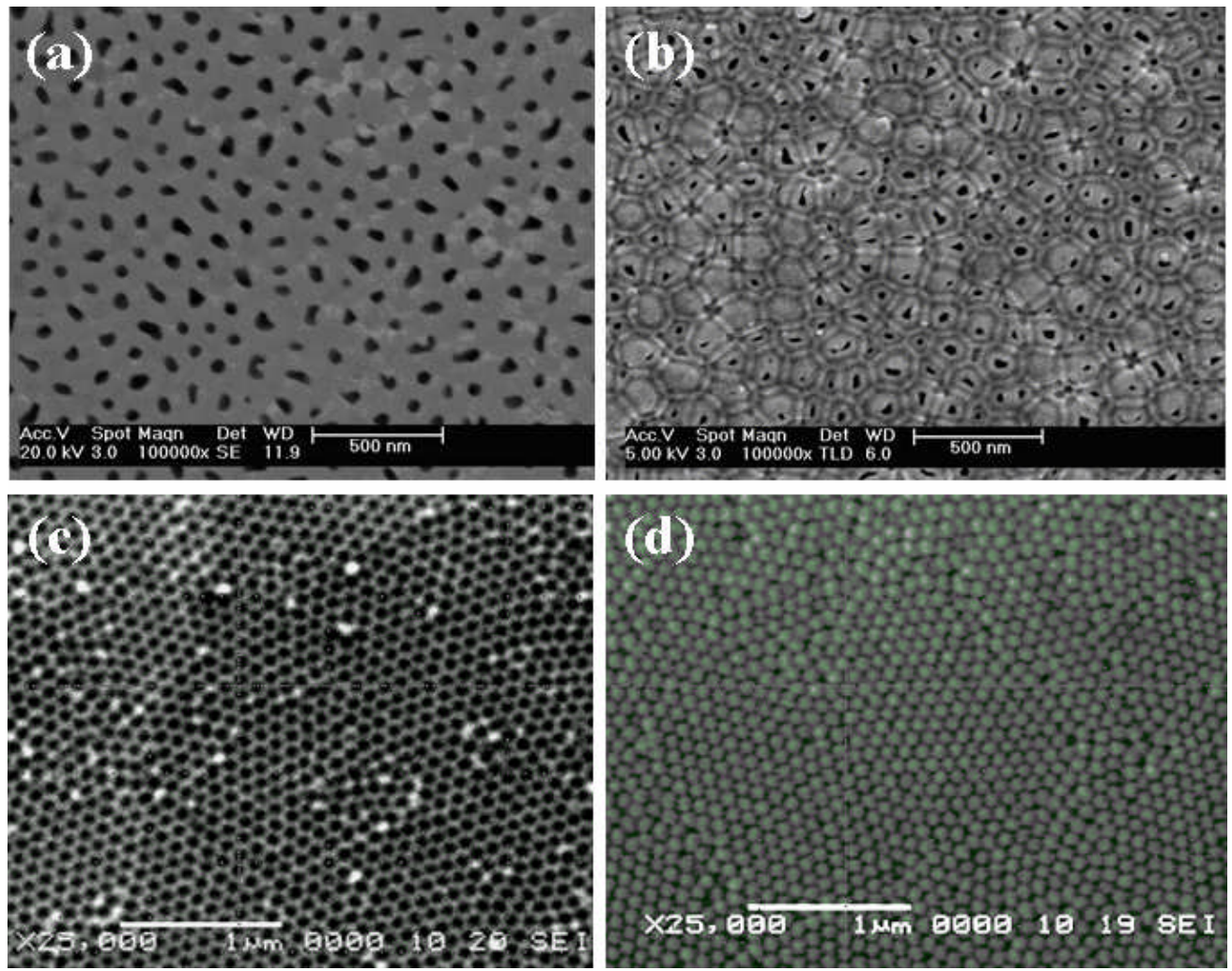

Figure 3-1. Top ( $a, c)$ and bottom (b, d) view SEM images for AAO prepared in $0.3 \mathrm{M}$ oxalic acid under $60 \mathrm{~V}(\mathrm{a}, \mathrm{b})$ and $40 \mathrm{~V}(\mathrm{c}, \mathrm{d})$, respectively. 

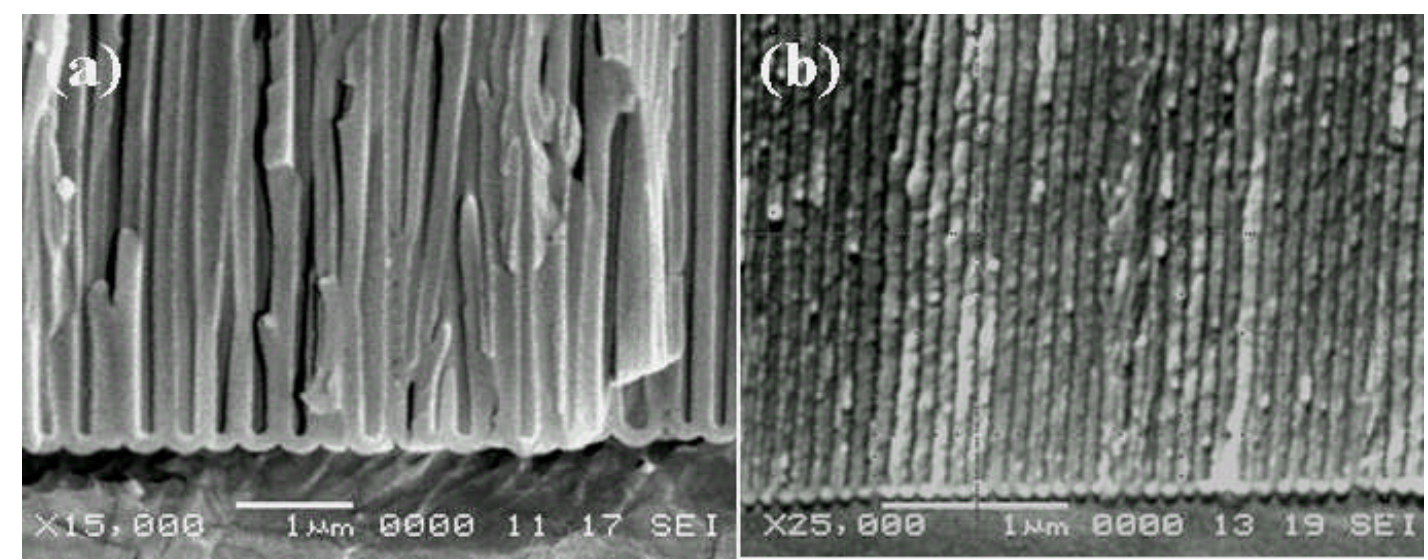

Figure 3-2. Sectional view SEM images for AAO prepared in $2 \mathrm{wt} \%$ phosphoric acid under 120 $\mathrm{V}(\mathrm{a})$, and $0.3 \mathrm{M}$ oxalic acid under $40 \mathrm{~V}$ (b), respectively.

During the course of our research on the formation mechanism of AAO and anodic titanium oxide (ATO), an equifield strength model was established to interpret the growth of single pore, self-adjustment of neighboring pores and the ordering of pore arrays. ${ }^{78,225}$ In the present work, we applied the equifield strength model to explain all the experimental observations during the formation of the porous AAO films, including pore merging, splitting and adjusting. It was also found that the relative dissolution rate of water during the anodisation is very important in the determination of the porosity. ${ }^{78,225}$ We elucidate that the relationship of the relative dissolution rate of water during the anodisation and the porosity can be used not only for the ordered pore arrays, but also for disordered pores in AAO. We also try to establish the relationships between the porosity of AAO films and the applied anodisation conditions.

\subsection{Equifield strength model and its application in the AAO formation}

\subsubsection{Anodic oxidation of Al plates}

Depending on the property of an electrolyte employed, two types of alumina barrier layer normally form during anodisation of aluminium. A nonporous planar layer forms in a near-neutral electrolyte, e.g., boric acid, ammonium borate, tartrate, and ammonium tetraborate, while a porous anodic aluminium oxide layer forms in weak-acidic electrolytes such as sulfuric, 
phosphoric and oxalic acids.

Chemical reactions during the anodisation of aluminium look simple. For example, the overall reaction can be written as $2 \mathrm{Al}+3 \mathrm{H}_{2} \mathrm{O} \rightarrow \mathrm{Al}_{2} \mathrm{O}_{3}+3 \mathrm{H}_{2}$. This reaction is correct, but does not reflect the details of the whole electrochemical process. It is essential to understand the chemical reactions at the electrolyte/oxide and oxide/metal interfaces separately. It is certain that, when an electric field is supplied, aluminium at the oxide/metal interface will be oxidized into $\mathrm{Al}^{3+}$ cations: $\mathrm{Al} \rightarrow \mathrm{Al}^{3+}+3 \mathrm{e}$. Some of these cations migrate across the oxide layer and are dissolved into the electrolyte (in a case of porous AAO) or form oxide at the electrolyte/oxide interface (in a case of nonporous AAO). Some of them stay at the oxide/metal interface, forming $\mathrm{Al}_{2} \mathrm{O}_{3}$ with the oxide anions migrated from the electrolyte/oxide interface.

There is still an unsolved problem regarding the above reactions, i.e. the form of the oxygen-containing anions is uncertain, as they could be $\mathrm{O}^{2-}$ or $\mathrm{OH}^{-}$. In the anodisation of titanium, it has been recently found that the wall of ATO nanotubes contains two layers with the outer layer of titanium hydroxide, implying that some anions must be $\mathrm{OH}^{-}{ }^{78}$ The hydroxide will eventually decompose to form oxide. In the case of $\mathrm{AAO}$, the dehydration of $\mathrm{Al}(\mathrm{OH})_{3}$ forming $\mathrm{Al}_{2} \mathrm{O}_{3}$ requires an energy of only $3.80 \mathrm{kcal} / \mathrm{mol}$. Even if hydroxide of aluminium forms as an intermediate phase, it will decompose very quickly and no detectable hydroxide layer appears.

It has been widely accepted that two basic chemical processes take place during the anodisation of aluminium: dissolution of $\mathrm{Al}_{2} \mathrm{O}_{3}$ at the electrolyte/oxide interface as shown in Figure 3-3a marked by A, B, C, and oxidation of aluminium at the interface of oxide/metal marked A', B', C'. At the electrolyte/oxide interface, $\mathrm{Al}_{2} \mathrm{O}_{3}$ is dissolved in electrolyte. The oxide anions are driven by the electric field to move across the oxide layer, contributing to the formation of $\mathrm{Al}_{2} \mathrm{O}_{3}$ at the oxide/metal interface. However, these oxide anions are not enough for the newly formed oxide layer. A significant amount of anions must be supplied from dissociation of water at the electrolyte/oxide interface. A water-splitting reaction: $\mathrm{H}_{2} \mathrm{O} \rightarrow 2 \mathrm{H}^{+}+\mathrm{O}^{2-}$ or $\mathrm{H}_{2} \mathrm{O}$ $\rightarrow \mathrm{H}^{+}+\mathrm{OH}^{-}$was therefore proposed. ${ }^{79,80}$ It was found in our work that dissociation of water not only supplies anions to the oxide layer, but also plays an important role in governing the porosity of the porous AAO films. 


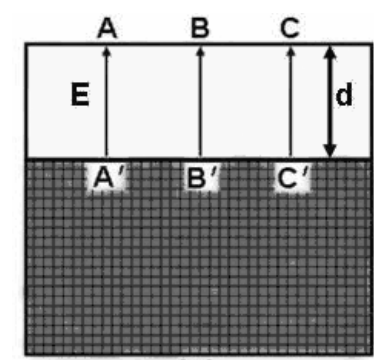

(a)

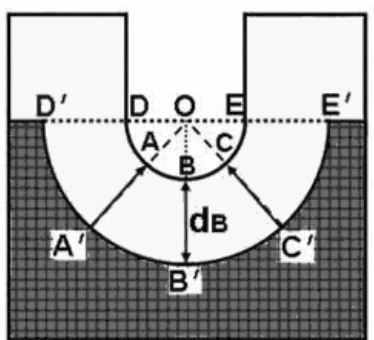

(d)

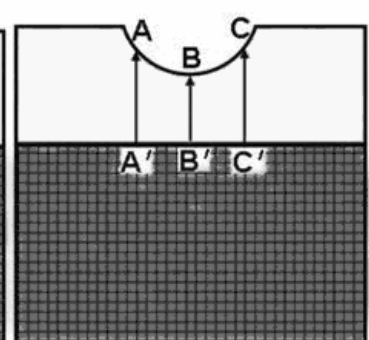

(b)

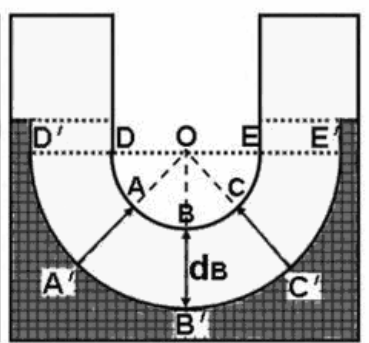

(e)

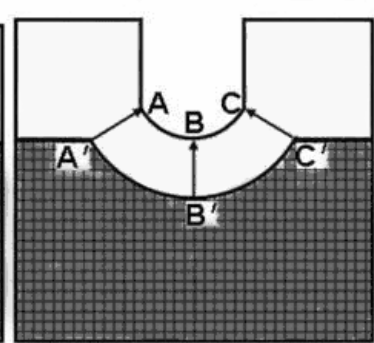

(c)

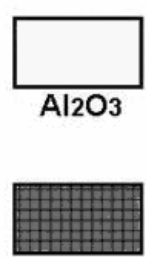

Al

Figure 3-3. Schematic diagrams for the electric-field strength distribution on some typical barrier layers, (a) planar layer with a uniform thickness, (b) planar layer with a corrosive pit, (c) a corrosive pit at the electrolyte/ oxide interface is replicated at the oxide/metal interface, (d) formation of the hemispherical pore base and (e) single pore formation. ${ }^{225}$

We believe that the following overall reaction for the dissolution of $\mathrm{Al}_{2} \mathrm{O}_{3}$ describes the newly proposed equifield strength model best and reveals the importance of the dissociation of water:

$\mathrm{Al}_{2} \mathrm{O}_{3}+n \mathrm{H}_{2} \mathrm{O} \rightarrow 2 \mathrm{Al}^{3+}+(3+n-x) \mathrm{O}^{2-}+x \mathrm{OH}^{-}+(2 n-x) \mathrm{H}^{+}$

where $n$ is used to indicate the ratio of the dissociation of water to the dissolution of $\mathrm{Al}_{2} \mathrm{O}_{3}$ which will be further discussed below, and $x$ indicates the ratio of $\mathrm{O}^{2-}$ and $\mathrm{OH}^{-}$, which has not yet been determined. ${ }^{225}$ Although the ratio of produced $\mathrm{O}^{2-}$ to $\mathrm{OH}^{-}$cannot be determined, we assume both of these anions migrate in the electric field from the solid surface to the oxide/metal interface, marked by A', B' and C' in Figure 3-3a and contribute to the following oxidation reactions of aluminium:

$$
\begin{aligned}
& 2 \mathrm{Al}+3 \mathrm{O}^{2-} \rightarrow 2 \mathrm{Al}_{2} \mathrm{O}_{3}+6 \mathrm{e}^{-} \\
& 2 \mathrm{Al}+3 \mathrm{OH}^{-} \rightarrow \mathrm{Al}_{2} \mathrm{O}_{3}+3 \mathrm{H}^{+}+6 \mathrm{e}^{-}
\end{aligned}
$$


Reaction (3.1) reduces the thickness of the oxide layer, while reactions (3.2) and (3.3) lead to an increase of the thickness of such a layer.

\subsubsection{Equifield strength model}

When aluminium is anodised in a near-neutral solution, the dissolution of $\mathrm{Al}_{2} \mathrm{O}_{3}$ according to reaction (3.1) is very slow. The thickness of the oxide layer will continuously increase following reactions (3.2) and (3.3). On the other hand, the electric field strength can be written as $E=U / d$. For a constant applied voltage $U$ to the oxide layer, the electric field strength $E$ in the oxide layer is inversely proportional to the thickness of the oxide layer, $d$. Therefore, $E$ will drop down when the thickness of the oxide layer increases, reducing the migration rate of the anions. The oxidation process will eventually stop when $d$ approaches a critical value, $d_{\text {Critical }}$ (abbreviated as $d_{C}$ hereafter), depending on the anodisation conditions, while the corresponding electric field strength, $E_{\text {Critical }}$ (abbreviated as $E_{C}$ hereafter) $=U / d_{C}$, is merely too weak to drive the oxygen containing anions through the oxide barrier layer. According to a previous study, the $U / d_{C}$ value is about $0.7 \mathrm{~V} / \mathrm{nm}$ for a near-neutral electrolyte. ${ }^{47}$

It is noted that, in a near-neutral electrolyte, wherever the thickness of the oxide layer is smaller than $d_{C}$, the field strength $E>E_{C}$, and the migration of anions will continue to thicken the oxide layer. Finally, a uniform thickness of $d_{C}$ and therefore constant electric field strength is achieved in the whole barrier layer.

When aluminium is anodised in an acidic solution, dissolution of $\mathrm{Al}_{2} \mathrm{O}_{3}$ at the oxide/electrolyte interface becomes significantly faster. Though an applied electric field can enhance both the dissolution of the oxide (reaction 3.1) and the inward oxygen-containing ionic current, and therefore the oxidation rate (reactions 3.2 and 3.3), it can be expected that the effect of the field to the latter process is greater than that to the former process. For the oxidation, a stronger field enhances the oxidation rate directly by a stronger electric force driving the oxygen anions through the oxide film. According to the high field conductivity theory applied to valve metals, ${ }^{226,227}$ the ionic current density $(j)$ is related to the effective electric field strength $(E)$ across the barrier layer (Tafel's Law): $j=j_{0} \exp (\beta E)$, where $\mathrm{j}_{0}$ and $\beta$ are material-dependent constants. 
The oxidation rate increases dramatically with increasing field strength. While for the dissolution of $\mathrm{Al}_{2} \mathrm{O}_{3}$, a stronger field results in more severe polarization and impairment of the Al-O bond of alumina, and then facilitates the dissolution. The dissolution rate of $\mathrm{Al}_{2} \mathrm{O}_{3}$ will increase with increasing field strength, but not as much as the increase of the oxidation rate. Since the anodisation current is dependent on the reactions (3.2) and (3.3), which are governed by the dissolution of $\mathrm{Al}_{2} \mathrm{O}_{3}$ and the dissociation of water according to reaction (3.1), it can be concluded that increasing $E$ largely accelerates the dissociation of water.

There always exists a critical value of the barrier thickness $\left(d_{B}\right)$ and the corresponding field strength $\left(E_{B}\right)$ to achieve a balance between the oxidation and the dissolution processes. When the barrier is thinner than $d_{B}$, the field strength $E$ is larger than $E_{B}$, the oxidation will surpass the dissolution, hence thicken the barrier layer. When the oxide layer is thicker than $d_{B}, E<E_{B}$, the dissolution will exceed the oxidation and the thickness of the oxide layer is reduced. Consequently, at a steady state, the thickness of the oxide layer $\left(d_{B}\right)$, oxidation rate and dissolution rate are all constant under certain anodisation conditions. The electric field strength $\left(E_{B}\right)$ across the barrier layer in the whole anodisation area is constant as well, demonstrating again the equifield strength state.

If the acidity of the electrolyte increases, the dissolution rate of the oxide layer increases. The thickness of the oxide layer $\left(d_{B}\right)$ will reduce accordingly, leading to an increase of $E_{B}$. Consequently, a new balance will be achieved with a thinner $d_{B}$ and larger $E_{B}$. Generally speaking, $E_{B}$ corresponding to an acidic electrolyte is normally larger than $E_{C}$ in a near-neutral condition when the applied voltages are the same. Therefore, unlike the anodisation in a near-neutral electrolyte, both the oxidation and dissolution processes in an acidic electrolyte continue indefinitely. ${ }^{25,47,70}$

\subsubsection{Pore initiation}

Corrosion of aluminium takes place during anodisation in an acidic electrolyte even if no electric field is applied. Considering the very early stage of the anodisation, however, the dissolution rate in the whole surface area is normally not even. Defects such as impurities, dislocation, grain boundaries, or nonmetallic inclusions in the underlying metal can cause a 
faster dissolution rate and lead to a pit growth (Figure 3-3b). ${ }^{228,229}$ It has been also reported that, when $\mathrm{Al}^{3+}$ cations are ejected from the oxide surface driven by an applied field, cation vacancies can arise and accumulate to form high density voids in the oxide film, which can also help the propagation of pits. ${ }^{230,231}$

On the other hand, aluminium forms a thin oxide layer in air and this layer is normally rough on a nanometer scale. A large number of defects in the layer can act as pit initiation sites and an applied voltage can increase the pit number and growth rate rapidly. This assumption was supported by Hebert and co-workers in their studies of anodic etching and pitting corrosion of aluminium respectively. ${ }^{232,233}$

In the present work, a non-pretreated aluminium foil was dipped in a fresh $0.3 \mathrm{M}$ oxalic acid for $20 \mathrm{~h}$ without any applied electric field. It was shown that high density pits from tens of nanometers to several hundred nanometers in diameter can be found over the whole surface as shown in Figure 3-4a. When a voltage of $40 \mathrm{~V}$ was applied, pores with a high concentration and a rather uniform size could be observed after only $90 \mathrm{~s}$ and the ordering of the pores increased with the anodisation time (Figure $3-4 b$ to $d$ ).

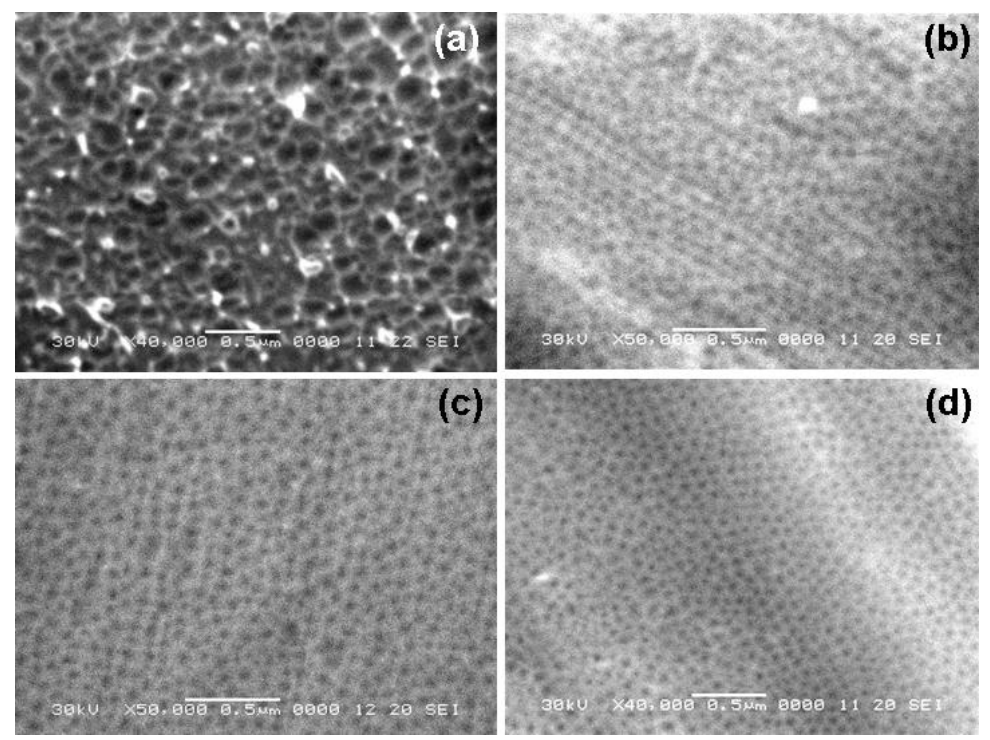

Figure 3-4. SEM images of an aluminium foil after dipping in $0.3 \mathrm{M}$ fresh oxalic acid for $20 \mathrm{~h}$ (a), base surfaces of aluminium substrate with alumina stripped away after anodisation at $40 \mathrm{~V}$ in $0.3 \mathrm{M}$ oxalic acid for $30 \mathrm{~s}$ (b), $60 \mathrm{~s}(\mathrm{c})$, and $90 \mathrm{~s}$ (d). 


\subsubsection{Growth of single pore}

When a pit forms on the oxide surface as shown in Figure 3-3b, the thickness of the oxide layer in this area becomes uneven. For example, the thickness at BB' in Figure 3-3b is smaller than that at $\mathrm{AA}^{\prime}$ and $\mathrm{CC}^{\prime}$, resulting in a relatively higher oxidation rate at the B' position. Consequently, the shape of the interface A'B'C' will intend to replicate that of $\mathrm{ABC}$ and the hemispherical shape shown in Figure 3-3c and 3-3d is the only one which can achieve an uniform thickness and, therefore, equifield strength in the whole area.

Consequently, when a single pore moves down to penetrate the aluminium plate, an ideal hemispherical pore bottom would be approached as shown in Figure 3-3d. The whole oxide/metal interface D'A'B'C'E' has a uniform field strength and so does the oxide/electrolyte interface DABCE. Further growth of the single pore as shown in Figure $3-3 \mathrm{e}$ leads to an appearance of a cylindrical interface above the hemispherical bottom. Since the field strength along DD' or EE' in the wall of the pore is the same as that at the bottom, the oxide layer can move not only downwards but also sidewards. In other words, a single pore may continuously increase its pore diameter to form a larger hemispherical bottom according to the equifield strength model, although in practice, this development is restricted by a high pore density. Further investigation indicated that the pore size is determined by the relative dissociation rate of water and the anodisation conditions. It is interesting to notice that other conditions influence the pore size via the relative dissociation rate of water. Nevertheless, this property is important for explaining some of the microstructures in AAO as discussed below.

\subsubsection{Self-adjustment of pores}

Since the pores are generated based on the pits which grow from inhomogeneity in the oxide film, a high density of pores is normally observed at a very early stage of anodisation and the ordering of the pores increases with the anodisation time, as demonstrated in Figure 3-4. We use the proposed equifield strength model to explain the self-adjustment of the pores and the improvement of the pore ordering in AAO during anodisation below.

To simplify the discussion, a two-pore model is used. When two pores are completely 
separated as shown in Figure 3-5a, then, as we discussed above the pores will expand and therefore, the neighboring walls of these two pores will move towards each other until two walls merge with a thickness of the combined wall $2 d_{B}$ (Figure 3-5b).

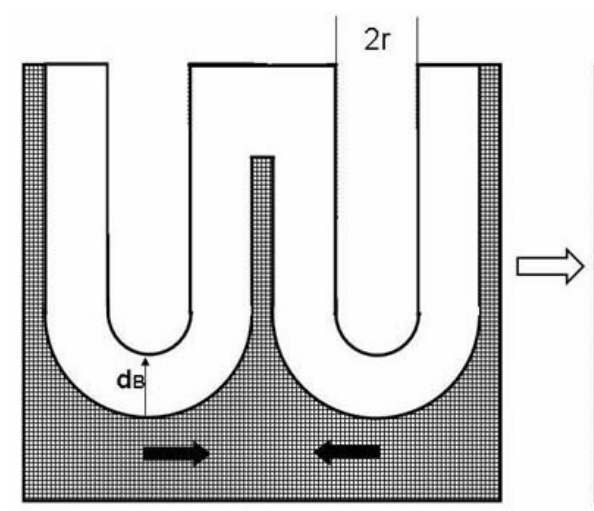

(a)

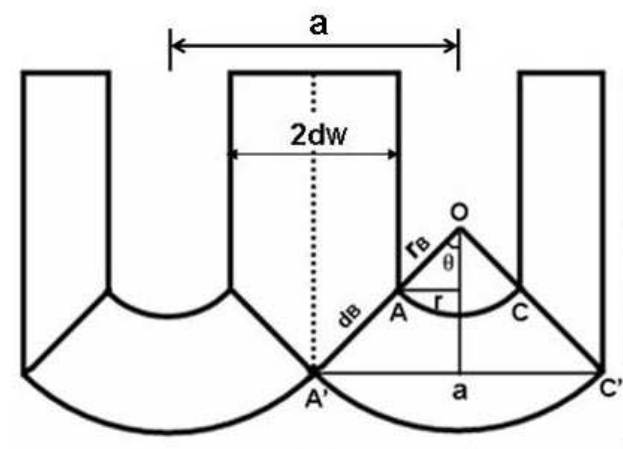

(c)

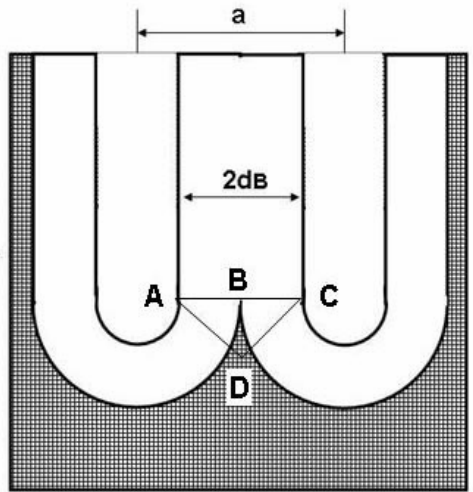

(b)

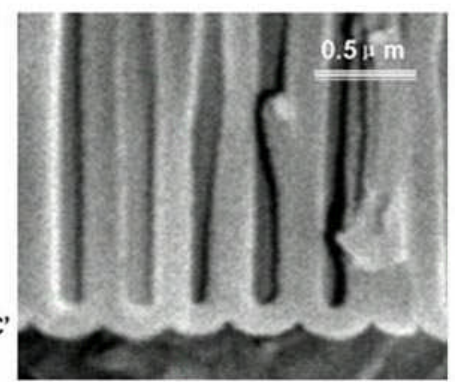

(d)

Figure 3-5. Schematic drawing showing that (a) two pores have a separation larger than $2 d_{B}$, (b) the pores move towards each other to achieve a wall thickness of $2 \mathrm{~d}_{\mathrm{B}}$, (c) pores move closer with a balanced curvature of $2 \theta$. (d) SEM profile image of pore bottom in an AAO film prepared at $120 \mathrm{~V}$ in $2 \mathrm{wt} \% \mathrm{H}_{3} \mathrm{PO}_{4}$ aqueous solution for $60 \mathrm{~min}$.

At this stage, both pore bottoms are still perfectly hemispherical. However this shape cannot be maintained during anodisation. The oxidation rate at the joined position of the hemispheres (B) must be much higher than at any other position because oxygen anions migrate from both sides. The joined position (B) will move down to a lower position (D) as shown in Figure 3-5b. In this case, the thickness of the oxide layer at position (D), i.e., $\mathrm{AD}$ or $\mathrm{CD}$, increases. The dissolution rate at positions $\mathrm{A}$ and $\mathrm{C}$ will be relatively faster than the oxidation rate at the position (D), 
leading to a reduction of the thickness of the oxide layer. A same oxidation rate in the whole oxide/metal interface area can eventually be maintained. In this case, the wall thickness between the pores, $2 d_{w}$, is smaller than $2 d_{B}$ (Figure 3-5c). The final shape of the pore bottom is therefore not perfectly hemispherical, but with a spherical angle $2 \theta$ which is smaller than $180^{\circ}$ as shown in Figure 3-5c.

This prediction can be confirmed by SEM images of the pore bottom as shown in Figure 3-5d. $\theta$ can be calculated from $d_{W}=d_{B} \sin \theta$, where $d_{W}=0.5 a-r$. For example, in a commonly used mild anodisation condition, $40 \mathrm{~V}$ in $0.3 \mathrm{M}$ oxalic acid for instance, $a=100 \mathrm{~nm}(2.5 \mathrm{~nm} / \mathrm{V}), r=$ $18 \mathrm{~nm}(0.45 \mathrm{~nm} / \mathrm{V})$ and $d_{B}=52 \mathrm{~nm}(1.3 \mathrm{~nm} / \mathrm{V})$, as observed experimentally, ${ }^{70}$ the corresponding $\theta$ is about $38^{\circ}$. The average $\theta$ value measured from the SEM image in Figure $3-5 \mathrm{~d}$ is similar to that value, although both $d_{B}(171 \mathrm{~nm})$ and $d_{W}(213 \mathrm{~nm})$ are much larger.

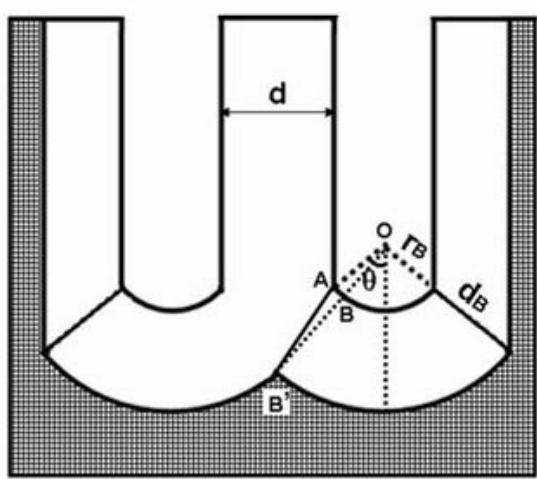

(a)

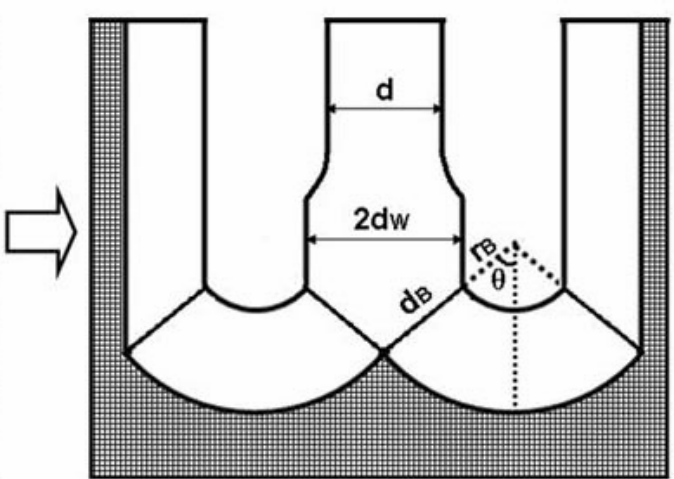

(b)

Figure 3-6. Schematic drawing of (a) two pores being too close to each other and (b) their self-adjustment to increase the wall thickness.

When two pores are too close to each other as shown in Figure 3-6a, $d<2 d_{w}$, the pores will move apart to increase the wall thickness as discussed below. The thickness along BB' is $d_{B}$, which is determined by the anodisation conditions. However, the thickness at any position in the range of $\mathrm{AB}$ to $\mathrm{B}$ ' is larger than $d_{B}$, leading to a lower field strength. The field-assisted oxide dissolution rate at these positions in the area $\mathrm{AB}$ becomes slower than that at the position $\mathrm{B}$ and below. An accumulation takes place in this area and the wall thickness between the pores will eventually be increased as shown in Figure 3-6b. This expansion process will stop when the wall 
thickness is $2 d_{w}$.
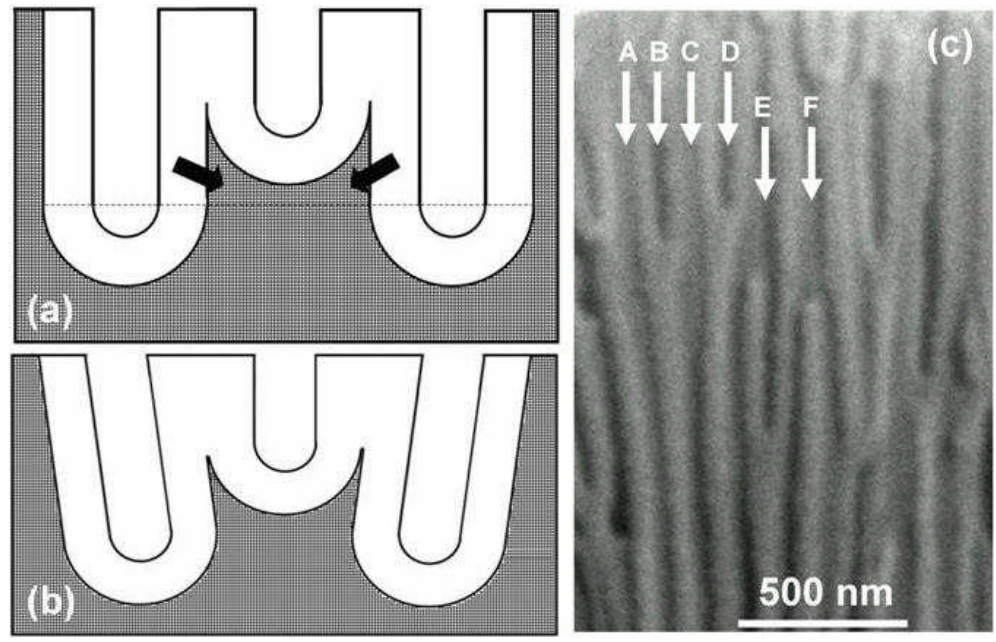

Figure 3-7. Schematic drawing of a short pore sandwiched by two long pores (a) and two pores on side move towards to restrict the growth of the pore in the middle (b). (c) SEM image showing disappearance of pores at early stage of anodisation.

According to the equifield strength model, the pore growth direction should be perpendicular to the surface of the oxide layer. In the case of a rough surface, the pores may not be parallel to each other as being often observed experimentally. The vertical divergences of the growth vector, in a macroscopic view, for anodic pores are different from each other. During anodisation, pores compete with each other since they have an interior tendency to enlarge their pore diameter and restrain the growth of their neighboring pores. Figure 3-7a shows three pores with the middle one growing relatively slower than the other two. In this case, the walls of two side pores will move towards the centre for the same reason as described in Figure 3-5 and the middle pore will reduce its pore size and its growth finally stops (Figure 3-7b). Such a disappearance of pores is often observed in the AAO film, especially at an early stage of pore growth, e.g. the pores marked as $\mathrm{B}$ and $\mathrm{D}$ in Figure 3-7c. Here, the contribution of mechanical stress may also be notable when two pores growing in different directions touch each other. The repulsive force between pores may help to form parallel arrays.

When the pores are relatively too large as marked by E and F in Figure 3-7c, they may split into two or more normal sized pores. The reason for such pore splitting is probably the constant 
ratio of the dissolution rate of $\mathrm{Al}_{2} \mathrm{O}_{3}$ and the dissociation rate of water, which will be discussed below. This property of self-adjustment of the pores, probably governed by a combined force of the equifield strength and the repulsion, leads to a parallel pore arrangement as observed in the lower part of Figure 3-7c.

The establishment of the equifield strength model allows us to explain the formation of pore morphology, potential expansion of the pores and the self-adjustment of pore ordering. In this way, after a sufficient long time of anodisation, well ordered hexagonal patterned AAO nanopore arrays can be achieved on the requirement of the equifield strength across the barrier oxide layer all over the oxide/metal interface.

\subsubsection{Impact factor on the ordering of AAO pore arrays}

On the above discussion, ion mobility under high electric field, the requirement of equifield strength all over the entire oxide/metal interface, mechanical stress induced by the expansion of oxide film during the oxidation of metal, and the plasticity of alumina work together to adjust the initially irregular pores to a close packed hexagonal array. From our equifield strength model, there is no particular limitation on the formation condition and the detailed pore parameters. This suggests that the hexagonal pore structure of AAO might be synthesized beyond the typical $25 \mathrm{~V}$ (sulfuric acid), $40 \mathrm{~V}$ (oxalic acid), $195 \mathrm{~V}$ (phosphorus acid) anodisation. Lee $e t a l^{70}$ successfully fabricated well ordered hexagonal alumina array with large aspect ratio with anodisation voltages range from $110 \mathrm{~V}$ to $150 \mathrm{~V}$. Other important works ${ }^{68,83,233}$ were also carried out to widen the successful condition for hexagonal patterning.

During the pattern formation, the temperature, type of electrolyte, and current density could all affect the quality of the pore ordering as well as the property of the aluminium substrate itself. Since the determined effect of the electric distribution on the barrier layer, the contribution of pretreatment such as electropolishing which only improve the surface quality should be rather limited and can be substituted by a short time anodisation. However, pretreatment of annealing will have a more significant effect since it improves the quality of the whole Al substrate by reducing the quantity of defects. In a recent work by Lo et al, ${ }^{224}$ it was found by experiment that annealing has a greater improvement on the pattern quality compared to electropolishing. 
Tolerable (avoiding burning and collapse) higher current density and local temperature could lead to larger domains because of stronger mechanical stress and better ion mobility. Certainly, there should be some adjustments between the electrolyte, temperature, applied voltage and current density to optimize the experimental results. This is supported by Ono et $a l^{94}$, in whose work it was found that the regularity of cell arrangement was higher at the center of the burnt area where extremely high current was locally concentrated. However, the authors concluded empirically that only the high electric field strength is the key controlling factor of self-ordering.

\subsection{Porosity of AAO}

\subsubsection{Relative dissociation of water and the porosity of AAO}

We demonstrated above that, to meet the requirement of equifield strength across the whole bottom of an AAO film, the thickness of the oxide layer has to be constant $\left(d_{B}\right)$ in a steady state, the pores can move horizontally via a self-enlargement process, and the optimal wall thickness between two pores is $2 d_{W}$. Applying this model to a $2 \mathrm{D}$ ordering, we can expect a close-packed hexagonal pattern of the pores. As commonly observed in synthesis, the average ordered hexagonal domain area is a linear function of the anodisation time and domains can grow as the anodisation prolonged. ${ }^{48}$ We mentioned that a single pore may increase its pore diameter continuously, but this process may be restricted by a high density of pores. In fact, it has been often observed experimentally that the cell dimension of the pores, i.e. pore diameter plus the wall thickness, is a function of the anodisation voltage. We found that the amount of oxygen anions from the electrolyte contributing to the oxidation of aluminium ( $n$ in reaction 3.1 ) plays a very important role in porosity.

We considered a single pore and its growth in time $\Delta t$ (Figure 3-8b). The wall thickness is $d_{W}$, which is a half of the wall thickness in the AAO film. During the pore growth, the whole pore bottom with the unchanged shape and the thickness of the oxide layer moves down. The only net change is an increase of the pore length of $\Delta L$. Assuming all the oxide anions from dissolution of $\mathrm{Al}_{2} \mathrm{O}_{3}$ at the electrolyte/oxide interface contribute to the formation of $\mathrm{Al}_{2} \mathrm{O}_{3}$ on the other side of the pore bottom, all the oxygen anions (total moles: $N_{o}$ ) created from the dissociation of water in 
reaction (3.1) migrates to the oxide/metal interface driven by the electric field and contribute to the oxidation of aluminium in the newly created wall corresponding to $\Delta L$. We then have

$$
N_{o}=\left(\frac{\sqrt{3}}{2} a^{2}-\pi \times r^{2}\right) \Delta L \times D_{o}
$$

where $D_{o}$ is the density of oxygen anions in $\mathrm{Al}_{2} \mathrm{O}_{3}$ and is about $0.094 \mathrm{~mol} / \mathrm{cm}^{3}$, calculated from the mass density of $\mathrm{Al}_{2} \mathrm{O}_{3}\left(3.2 \mathrm{~g} / \mathrm{cm}^{3}\right){ }^{84}$
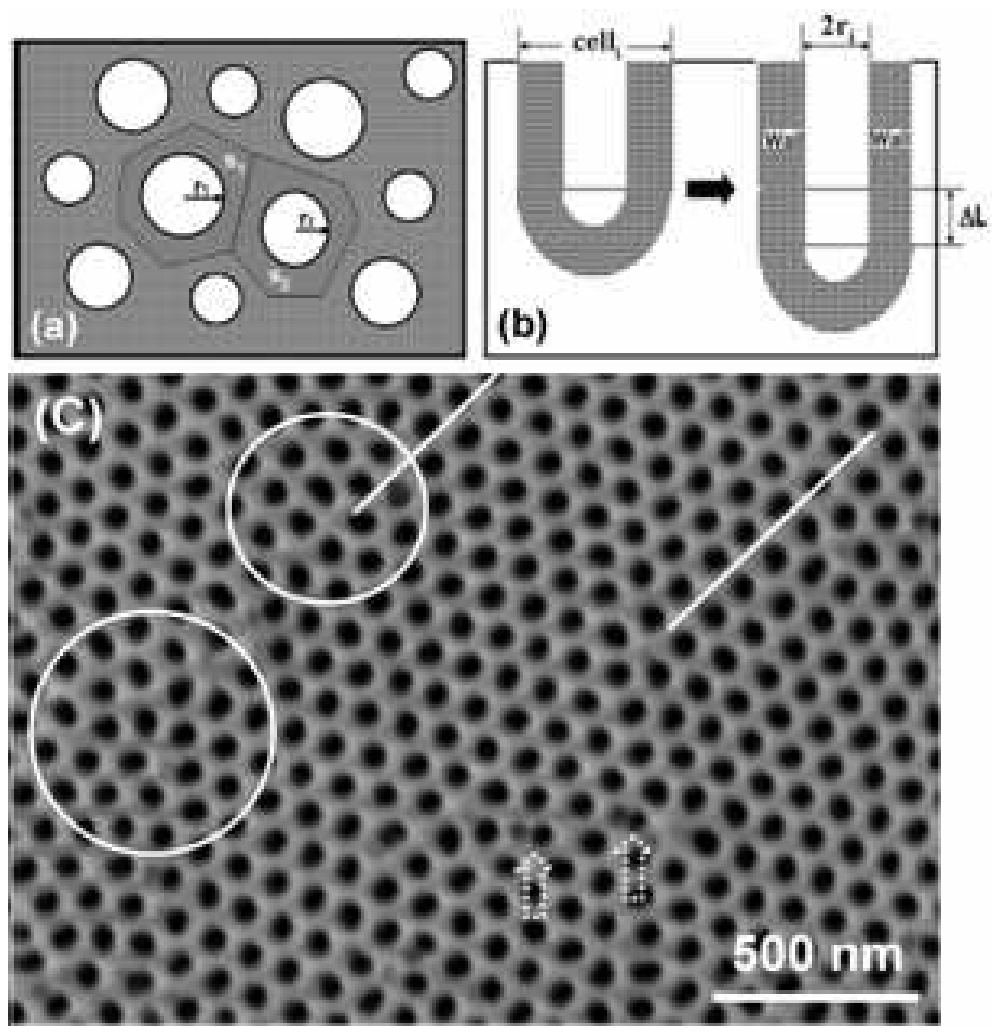

Figure 3-8. (a) Schematic drawing of a disordered pore arrangement in AAO, in which a cell area can be drawn for each pore. (b) Schematic drawing of a single pore growth for a length increase of $\Delta L$. (c) SEM image of an AAO film prepared by two-step anodisation: an aluminium plate was anodised in $0.3 \mathrm{M}$ oxalic acid at $40 \mathrm{~V}$ for $3 \mathrm{~h}$, the porous oxide layer was removed by a mixed solution of chromic and phosphorus acid, and the plate was anodised again for $10 \mathrm{~h}$.

During the same time of $\Delta t$, oxygen anions are produced via the dissolution of $\mathrm{Al}_{2} \mathrm{O}_{3}$ at the oxide/electrolyte interface. The total mole number of aluminium dissolved during the time of $\Delta t$ 
is $\pi r^{2} \Delta L \times D_{A l}$, where $D_{A l}$ is the molar density of $\mathrm{Al}$ cations in $\mathrm{Al}_{2} \mathrm{O}_{3}$ and has a value about 0.0627 $\mathrm{mol} / \mathrm{cm}^{3}$ according to the mass density of $\mathrm{Al}_{2} \mathrm{O}_{3}$ mentioned above. Since the molar ratio of oxide anions from the dissociation of water to the aluminium cations dissolved is $n / 2$, we have

$$
N_{o}=\left(\frac{\sqrt{3}}{2} a^{2}-\pi \times r^{2}\right) \Delta L \times D_{o}=\left(\frac{n}{2}\right) \pi \times r^{2} \times \Delta L \times D_{A l}
$$

Since $D_{O} / D_{A l}=3 / 2$,

$$
\left(\frac{r^{2}}{a^{2}}\right)=\frac{3 \sqrt{3}}{2 \pi(n+3)}
$$

Therefore, in a hexagonally ordered AAO film, the porosity has a relation with $n$ :

$$
p=\frac{2 \pi}{\sqrt{3}}\left(\frac{r}{a}\right)^{2}=\frac{3}{n+3} .
$$

We further demonstrate that this relationship can be universally applied to an AAO film with disordered pores. This is because the relative dissociation rate of water directly governs the ratio of the pore size to the cell dimension, rather than the positions of the pores.

Let us consider a disordered pore array as illustrated in Figure 3-8a,b. The pore sizes can also be different. For a single pore, $i$, it is always possible to draw a cell with an area of $S_{i}$ and the whole area of AAO is a sum of all the cell areas, $\Sigma S_{i}$. Accordingly, a cell contains a pore and a wall, although the latter can be irregularly shaped as shown in Figure 3-8a,b. Therefore, a schematic drawing of the pore growth of $\Delta L$ corresponding to the anodisation time $\Delta t$ is similar to that shown in the hexagonal model (Figure 3-8b).

The total number of moles of oxygen anions from the dissociation of water contributing to the oxidation of aluminium during $\Delta t$ in the cell $i$, is 


$$
N_{i}=\left(S_{i}-\pi \times r_{i}^{2}\right) \Delta L \times D_{o}=\left(\frac{n}{2}\right) \times \pi \times r_{i}^{2} \Delta L \times D_{A l} .
$$

where $S_{i}$ is the area of the cell. Therefore, the cell area is

$$
S_{i}=\frac{n+3}{3} \pi \times r_{i}^{2}
$$

and the porosity of this cell is

$$
P_{i}=\frac{\pi \times r_{i}^{2}}{S_{i}}=\frac{3}{n+3} .
$$

The $n$ value is dependant on the electric field strength. In a steady state, the electric field strength is constant according to the proposed equifield strength model. The $n$ value is therefore a constant for all cells. The total porosity, $P$, is also constant and has the same value as $P_{i}$ :

$$
P=\frac{\sum \pi \times r_{i}^{2}}{\sum S_{i}}=P_{i}=\frac{3}{n+3}
$$

This relation is independent of the shape of the pores. For pores with different shapes, the area of pores can be generally marked as $S_{p i}$ instead of $\pi \times r^{2}$. The final result of porosity is the same.

From the plot of $p$ against $n$ in Figure 3-9, we can see the porosity of AAO can vary, in principle, in a large range. The $10 \%$ rule proposed previously ${ }^{84}$ has no special physical meaning, except that the porosity near $10 \%$, corresponding to $n=27$, is not very sensitive to the change of $n$.

It can be expected that, under a stronger electric field, the dissociation rate of water and the anion current density will increase. The porosity will be reduced. As reported by Ono et al, ${ }^{93}$ the porosity decreased with rising applied voltage and current density from $P \sim 35 \%$ at $5 \mathrm{~V}$ and $\sim 1$ $\mathrm{mA}$ to $P \sim 10 \%$ at $40 \mathrm{~V}$ and $\sim 5 \mathrm{~mA}$ when aluminium was anodised in a $0.3 \mathrm{M}$ oxalic acid 
solution. This decrease of porosity can be regarded as a consequence of an increase of the $n$ value from 5.6 to 27 . It should not be too surprising that when Lee et al ${ }^{70}$ applied very high anodising voltage and current density, e.g., 120-150 V and 30-250 mA, they produced long-range ordered AAO with a very low porosity, $~ 3.3 \%$. Using the plot in Figure 3-9, this result can be explained with an increase of the $n$ value to about 88 .

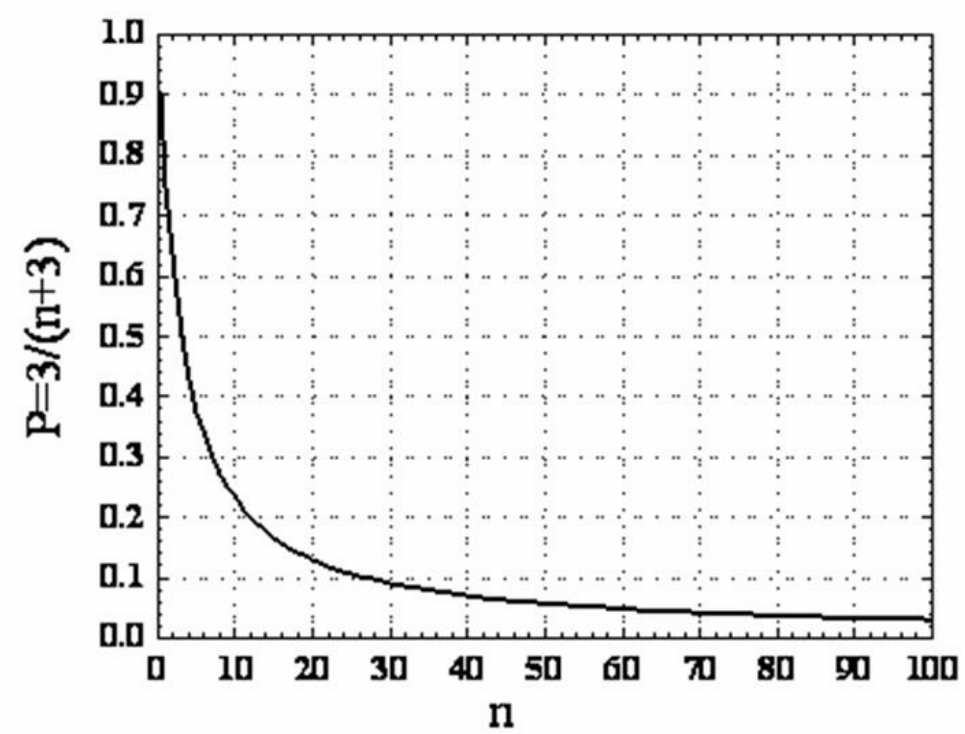

Figure 3-9. Porosity of $\mathrm{AAO}$ as a function of the relative rate of water dissociation at the oxide/electrolyte interface, $n$.

Figure 3-8c is a typical SEM image of AAO showing hexagonally ordered domains with some disordered regions. It is noticed that in the grain boundaries, e.g. a region marked by lines, the wall thickness might be larger than that in the ordered regions and the pores near these grain boundaries tend to be slightly larger as well to maintain a constant porosity. In the areas where the pore density is low, an enlargement of pore becomes obvious as indicated by circles. Alternatively, extra pores may appear as indicated by arrows in the lower part of the image.

\subsubsection{Ratio of dissolved Al cations from the two interfaces}

From the above analysis, we can see that the total mole number of aluminium oxidized in the oxide/metal interface corresponds to the total mole number of oxygen anions migrating to that 
interface, the latter being the sum of the mole number of water dissociated $(n)$ and 3 moles according to the $\mathrm{Al}_{2} \mathrm{O}_{3}$ dissolution. The proposed model also allows us to estimate the ratio of the mole number of $\mathrm{Al}^{3+}$ cations dissolved from $\mathrm{Al}_{2} \mathrm{O}_{3}$ in the pore bottom to the electrolyte $\left(A l_{\text {diss }}\right)$ to the total moles of aluminium lost $\left(A l_{\text {lost }}\right)$, the latter being the sum of $A l_{\text {diss }}$ and the moles of $\mathrm{Al}^{3+}$ directly ejected from the oxide/metal interface to the electrolyte $\left(A l_{\text {eject }}\right)$. Using the hexagonal model,

$$
\frac{A l_{\text {diss }}}{A l_{\text {lost }}}=\frac{\pi \times r^{2} \times \Delta L \times D_{A l(O)}}{\frac{\sqrt{3}}{2} a^{2} \times \Delta L \times D_{A l(M)}-\left(\frac{\sqrt{3}}{2} a^{2}-\pi \times r^{2}\right) \times \Delta L \times D_{A l(O)}}
$$

where $D_{A l(M)}$ is the molar density of $\mathrm{Al}$ in metal $\left(0.100 \mathrm{~mol} / \mathrm{cm}^{3}\right), D_{A l(O)}$ is the molar density of Al in oxide $\left(0.0627 \mathrm{~mol} / \mathrm{cm}^{3}\right)$. Replacing $a$ and $r$ according to equation (3.6),

$$
\frac{A l_{\text {diss }}}{A l_{\text {lost }}}=\frac{1}{1.594+0.198 n} \text {. }
$$

In a mild anodisation when the porosity is about $10 \%$ and the corresponding $n$ equals 27 , only $14.4 \%$ of the total $\mathrm{Al}^{3+}$ cations moving to the electrolyte are from the dissolution of $\mathrm{Al}_{2} \mathrm{O}_{3}$ in the oxide/electrolyte interface. $85.6 \%$ of the $\mathrm{Al}^{3+}$ cations are ejected directly from the oxide/metal interface without forming oxide. This estimation is in accordance with the previous experimental observations. $^{221}$

In the above calculation, volume expansion during the oxidation of aluminium has not been considered. If this factor is considered, according to the thickness $(\Delta L)$ of aluminium metal oxidized, the newly formed $\mathrm{Al}_{2} \mathrm{O}_{3}$ wall has a dimension of $\Delta L^{\prime}=k \Delta L$, where $k$ is the expansion factor along the pore growth direction. In this case,

$$
\frac{A l_{\text {diss }}}{A l_{\text {lost }}}=\frac{\pi \times r^{2} \times k \Delta L \times D_{A l(O)}}{\frac{\sqrt{3}}{2} a^{2} \times \Delta L \times D_{A l(M)}-\left(\frac{\sqrt{3}}{2} a^{2}-\pi \times r^{2}\right) \times k \Delta L \times D_{A l(O)}} \text {, that is }
$$




$$
\frac{A l_{\text {diss }}}{A l_{\text {lost }}}=\frac{k}{1.594+\left(0.532-\frac{k}{3}\right) n}
$$

The volume expansion factor varies over a large range from negative values to $1.6 .{ }^{27}$ If $k=1.2$, and $n=27$ for a commonly applied synthesis method, $23.3 \%$ of the total $\mathrm{Al}^{3+}$ cations moving to the electrolyte come from the dissolution of $\mathrm{Al}_{2} \mathrm{O}_{3}$ and $76.7 \%$ of the $\mathrm{Al}^{3+}$ cations are ejected directly from the oxide/metal interface without forming oxide.

\subsubsection{Relations between the porosity and anodisation conditions}

The oxygen anionic current across the barrier oxide at the pore base consists of two parts, $j_{\text {oxide }}$ from the electric field enhanced dissolution of the oxide at the oxide/electrolyte interface, and $j_{\text {water }}$ from the dissociation of water. To simplify the calculation, here we assume all the water molecules dissociate into protons and oxygen anions. We then have

$$
j=j_{\text {oxide }}+j_{\text {water }}
$$

where $j_{\text {water }} / j_{\text {oxide }}=n / 3$, since the current density is proportional to the anions created from the surface reactions. The porosity of the anodic oxide film should have a relation with these current densities,

$$
P=\frac{3}{n+3}=\frac{j_{\text {oxide }}}{j_{\text {oxide }}+j_{\text {water }}}=\frac{j_{\text {oxide }}}{j}
$$

Considering Tafel's Law, $j=j_{0} \exp (\beta U / d)=j_{0} \exp (\beta E), j_{0}$ and $\beta$ can be estimated from experiments. For example, when hard anodisation was carried out in $0.3 \mathrm{M}$ oxalic acid at $110 \mathrm{~V}$, a current density of $30 \mathrm{~mA}(j)$ with a barrier thickness $(1 / E)$ of $\sim 1.0 \mathrm{~nm} / \mathrm{V}$ were observed, while at $40 \mathrm{~V}, 5 \mathrm{~mA}$ and $\sim 1.2 \mathrm{~nm} / \mathrm{V}$ were measured. ${ }^{70,222}$ Substituting the experimental data to the eq. (3.16), we have $j_{0}=7.94 \times 10^{-4} \mathrm{~mA}$ and $\beta=10.54 \mathrm{~nm} / \mathrm{V}$. Thus, 
$j=7.94 \times 10^{-4} \exp (10.54 E)$

According to Parkhutik and Shershulsky, ${ }^{90}$ the electric current that is due to the aluminium oxide dissolution at the barrier oxide surface at the pore base has an exponential relation with the electric field strength. Therefore we can write $j_{\text {oxide }}=A \exp (k E)$, where $A$ is the pre-exponential factor for dissolution reactions and the coefficient $k$ depends on the working temperature and material property. Neglecting the current induced local heating of the barrier oxide at the pore base, for fixed anodisation conditions, e.g., $0.3 \mathrm{M}$ oxalic acid at temperature of $15{ }^{\circ} \mathrm{C}$ used in the present work, A and $\alpha$ can be treated as constants. Using the experimental data reported previously by Lee $e t a l^{70}$, the porosities of AAO anodised in $0.3 \mathrm{M}$ oxalic acid at $30 \mathrm{~mA}$ and 5 mA were found to be about $3.3 \%$ and $10 \%$ respectively. Substituting these values into eq. (3.16), we obtain

$j_{\text {oxide }}=1.69 \times 10^{-2} \exp (4.08 E)$

Then the porosity $P$ can be written as

$P=\frac{j_{\text {oxide }}}{j}=\frac{A \exp (k E)}{j_{0} \exp (\beta E)}=\frac{1.69 \times 10^{-2} \exp (4.08 E)}{7.94 \times 10^{-4} \exp (10.54 E)}=21.3 \exp (-6.46 E)$

The corresponding $P \sim E$ plot together with some of the previously reported experimental data is shown in Figure 3-10a. A rather good match can be observed.

From equations (3.17) and (3.19), the relationship between the porosity and the ionic current density $(j)$ can be deduced as

$$
p=21.3 \times\left(\frac{7.94 \times 10^{-4}}{j}\right)^{0.613}
$$

and is plotted in Figure 3-10b. 

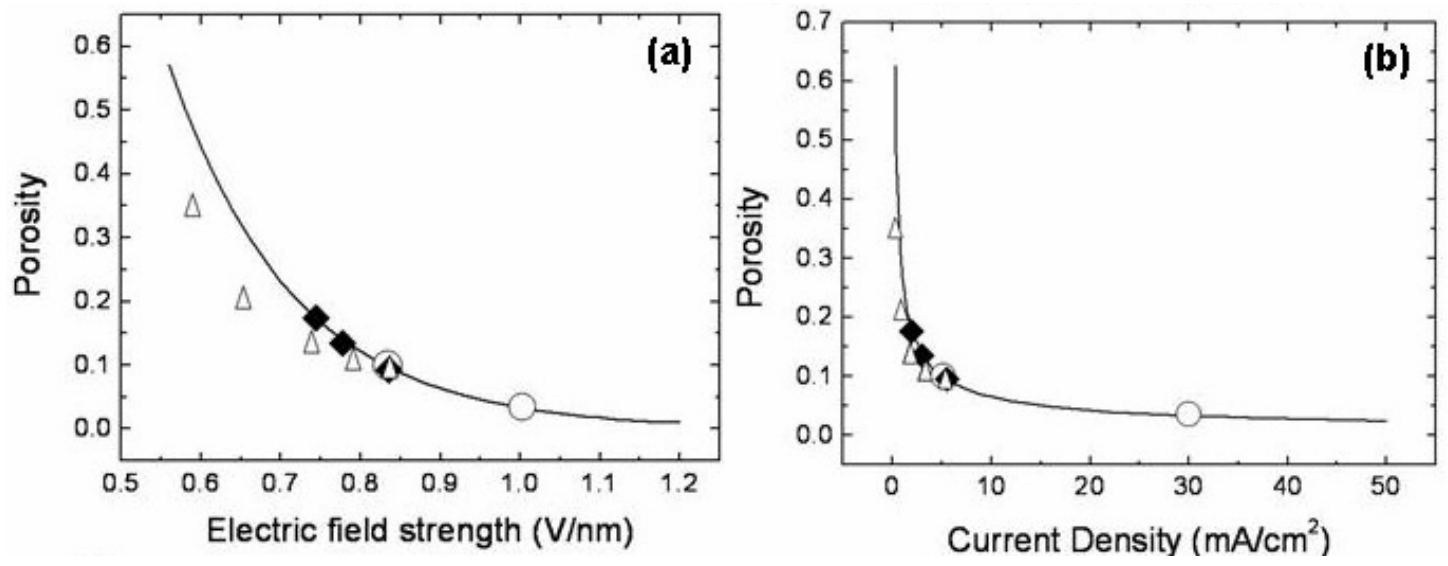

Figure 3-10. (a) Porosity $(P)$ of AAO forming in $0.3 \mathrm{M}$ oxalic acid as a function of the electric field strength $(E)$ across the oxide layer at the pore base. (b) Porosity $(P)$ of AAO within the same electrolyte as a function of the ionic current density $(j)$. The triangles represent the experimental data from reference [93], the circles from references [70, 235] and the solid diamonds from the present work.

The relationship between the porosity and the applied voltage is more useful than that between the porosity and the field strength from an experimental point of view, since the applied voltage is what is directly known from the experiments. However, in Tafel's Law, $j=j_{0} \exp (\beta U / d)$, where $U / d=E$, we do not know whether $j$ has an exponential relation with $U$, since $j$ is also a function of $d$.

If we accept Ono et al's ${ }^{93}$ assumption that the current density increases exponentially with the applied voltage based on their observation using three electrolytes: oxalic acid, sulfuric acid and phosphorus acid, we can write

$$
j=j_{0}{ }^{\prime} \exp (\alpha U)
$$

Substituting the experimental data from Lee et $a l,{ }^{70} 30 \mathrm{~mA}$ at $110 \mathrm{~V}$ and $5 \mathrm{~mA}$ at $40 \mathrm{~V}$, into the above equation, we obtain $j_{0}{ }^{\prime}=1.80 \mathrm{~mA}$ and $\alpha=0.0256 \mathrm{~V}^{-1}$. 
A combination of eqs. (3.21) and (3.17) enables us to derive a relationship between the thickness of the oxide layer and the applied voltage:

$d=\frac{\beta}{\alpha}\left(1-\frac{\ln \frac{j_{0}{ }^{\prime}}{j_{0}}}{\ln \frac{j_{0}{ }^{\prime}}{j_{0}}+\alpha U}\right)=411.7-\frac{3180.8}{7.726+0.0256 U}$

Note that equation (3.22) predicts an oxide layer thickness of zero if no voltage is applied. On the other hand, a combination of eqs. (3.20) and (3.21) gives a $P \sim U$ relation,

$P=21.3 \times\left[\frac{4.41 \times 10^{-4}}{\exp (0.0256 U)}\right]^{0.613}$

Figure 3-11 shows the plots of oxide layer thickness $d$ versus $U$ and $P$ versus $U$ together with previously reported experimental data ${ }^{70,93,235}$ and the data obtained in the present work.
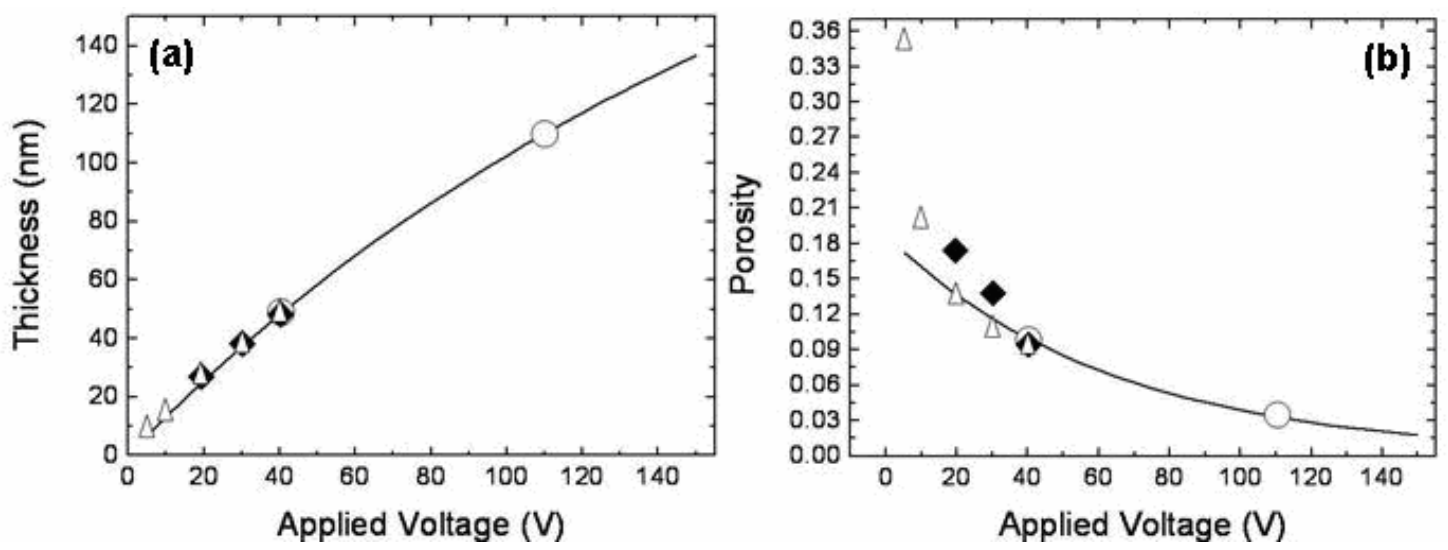

Figure 3-11. (a) Thickness of oxide layer versus applied voltage and (b) porosity of AAO versus applied voltage in $0.3 \mathrm{M}$ oxalic acid, when $j$ has an exponential relation with $U$. The triangles represent the experimental data from reference [93], the circles from references [70,235] and the solid diamonds from the present work.

In another consideration, we know that the oxide layer thickness will be finite even at very low voltages. We can model the relation between the thickness of the oxide layer and the voltage 
according to equation (3.24),

$d=d_{\text {final }}(1-\exp (-\gamma U))+d_{0}$

where $d_{0}$ describes the thickness of the native oxide layer when no voltage is applied, $d_{f i n a l}$ is a measure of the maximum thickness, and $\gamma$ describes the increase of the thickness with $U$. Combining eqs. (3.24) and (3.19) gives the relation between $P$ and $U$ according to.

$P=21.3 \exp \left(-6.46 \times \frac{U}{d_{\text {final }}\left(1-\exp (-\gamma U)+d_{0}\right.}\right)$

Using the experimental data by Ono et $a l^{93}$ and Lee et $a l,{ }^{70}$ we have fixed $d_{0}$ and fitted $d_{f i n a l}$ and $\gamma$. We have then inserted this into equation (3.19) and compared the curve with the experimental data. Best results were obtained with $d_{0}=1.5 \mathrm{~nm}, d_{\text {final }}=236.4 \mathrm{~nm}$, and $\gamma=$ $5.6 \times 10^{-3} \mathrm{~V}^{-1}$.
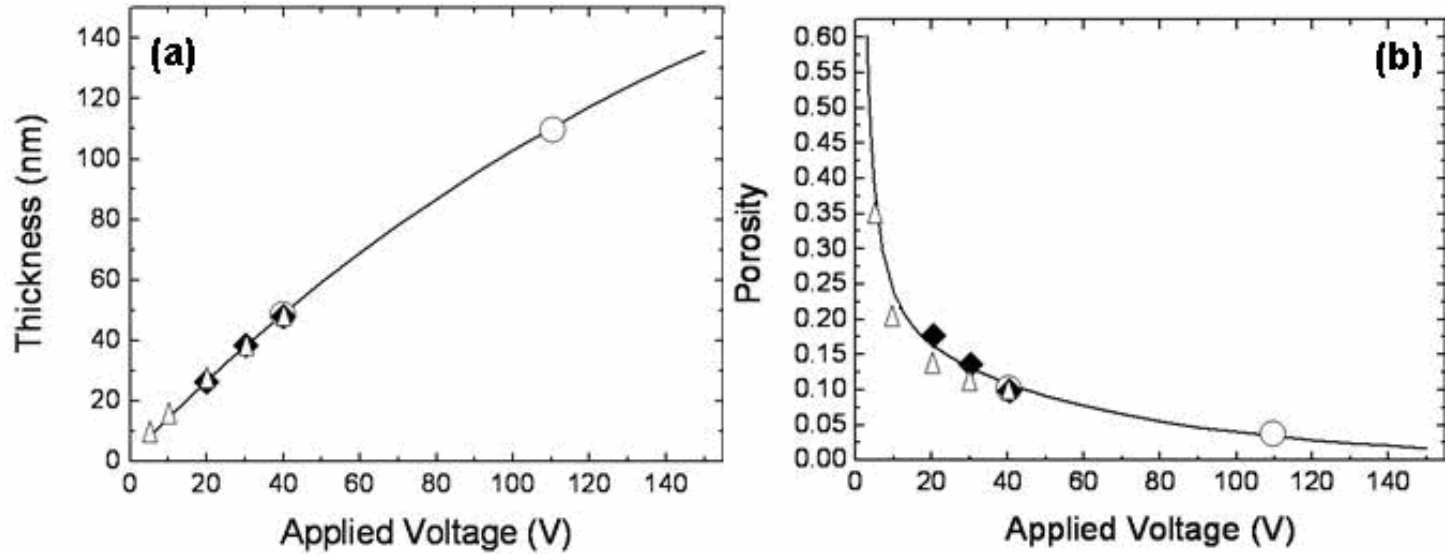

Figure 3-12. (a) Thickness of oxide layer versus applied voltage and (b) porosity of AAO versus applied voltage in $0.3 \mathrm{M}$ oxalic acid, when $\mathrm{d}$ has an exponential relation with $U$. The triangles represent the experimental data from reference [93], the circles from references [70,235] and the solid diamonds from the present work.

The dependences of $d$ and $P$ on $U$ are shown in Figure 3-12. Note that the increase of the 
thickness with voltage is virtually identical to that in Figure 3-11. However, the parameter $d_{0}$ is crucial. In fact, setting $d_{0}=0$ leads to an almost identical result for the $P$ vs $U$ graph as shown in Figure 3-11b. In that case the experimental data are described quite well for values above $20 \mathrm{~V}$, but the model cannot fit the data over the full range of experimental voltages reported in the literature, in particular those below $10 \mathrm{~V}$. With equation (3.24) taking the finite thickness of the oxide layer at small voltages into account a very good fit over the entire set of voltages is obtained.

In the present work, the establishment of an equifield strength model allows us to further understand the formation mechanism of porous AAO films, including potential expansion of a single pore, self-adjustment of pore ordering, pore merging and splitting, and formation of a spherical segment morphology of the pore bottom. It has been found that the dissociation rate of water for supplying oxygen anions to the oxide/metal interface is the most important driving force for adjusting the porosity of AAO, or the ratio of the pore size to the cell dimension. Consequently, this model including the contribution of the dissociation of water to the porosity opens a new avenue to reveal the pore formation in AAO.

We also simulated the relations between the porosity of AAO and anodisation conditions, such as field strength, applied voltage and current density using different approaches. It was found that taking the final thickness of the oxide layer at very low voltages into account is crucial (see Figure 3-11b and 3-12b). The resulting plot (Figure 3-12b) allows us to better control the structures of the porous AAO films, although some degree of mismatching needs further investigation. For example, in reality, the dissolution rate of the oxide may increase significantly with local temperature rise, leading to an increase of the ionic current density. Further deviation from the experimental data could occur due to a loss of oxygen anions when they are oxidized into $\mathrm{O}_{2}$ bubbles on the electrolyte/oxide interface. In addition, the ratio of dissolved $\mathrm{Al}^{3+}$ into the electrolyte from the electrolyte/oxide and oxide/metal interfaces has been calculated using the newly established model.

\subsection{Pore size variation with voltage of AAO films}

Investigations on the morphology parameters of the anodic films were extensively performed 
after the confirmation of the classical hexagonal cell model proposed by Keller et al in 1953. ${ }^{24,25,}$ 70, 235,236 According to Keller's reports ${ }^{24}$ on the AAO, the cell size of anodic oxide coatings formed at any voltage can be calculated from the equation: $C=2 W E+P$, where $C$ is the size of individual oxide cell, $W$ is the thickness of pore wall in an individual cell per volt, $E$ is forming voltage, and $P$ is pore diameter. It was believed that the thickness of the oxide wall increased linearly with voltage and the pore diameter remains constant. The thickness of the barrier layer also dependent on the anodising voltage linearly: $T=B E$, where $T$ is the barrier thickness and $B$ is the barrier thickness per volt. ${ }^{235}$ Later extensive studies suggested that the pore diameter of AAO primarily depended on the formation voltage also and was directly proportional with the anodising voltage as well as the cell diameters and barrier layer thickness, ${ }^{25,70,236}$ although the constant of proportionality would be expected to vary slightly with the anodising conditions.

As it has been confirmed that formation temperatures, electrolyte types and current densities could all affect the pore diameter and the barrier thickness in certain extent. ${ }^{25}$ Ono et $a l^{237}$ found that pore diameters of AAO formed at an identical voltage in different electrolytes are larger in the order: sulfuric acid < oxalic acid $<$ chromic acid $<$ phosphoric acid. Pore diameter at the film surface was larger than that at the pore base next to the barrier layer due to the chemical dissolution of anodic films in the electrolytes. In the formation of porous AAO films, the pure chemical dissolution rate of the anodic oxide film in the commonly used electrolyte is rather slow without the presence of electric field. The chemical dissolution of the anodic oxide film in the electrolyte has little impact on the morphology of the produced AAO film.

Recently, based on the equilibrium between the oxidation of aluminium metal caused by the inward oxygen-containing ions $\left(\mathrm{O}^{2-}\right.$ and $\mathrm{OH}^{-}$, mainly produced from dissociation of water) and the dissolution of the $\mathrm{Al}_{2} \mathrm{O}_{3}$ film produced, we proposed an equifield strength model to explain the self-organisation of highly ordered pores array in AAO ${ }^{78,225}$ Field enhanced dissociation of water was found to be important in the determination of the structure of anodic oxide films. It was established that the porosity of anodic oxide films was governed by the relative dissociation rate of water, which is dependent on anodisation parameters such as electrolyte, applied voltage, current density, and electric field strength. This model revealed an approximate exponential relationship, rather than the linear one as proposed previously, between the barrier thickness, porosity and the anodising voltage in a certain electrolyte when the temperature effect is 
neglected. In the present work, the dependence of pore diameter of AAO films on the applied voltages was also studied.

Supposed all oxide anions from the dissolution of $\mathrm{Al}_{2} \mathrm{O}_{3}$ at the pore base migrate to other side of the oxide layer to reform $\mathrm{Al}_{2} \mathrm{O}_{3}$ and all the oxide anions from the dissociation of water contribute to the construction of the wall, the porosity $P$ of AAO has a simple relation with the relative dissociation rate of water $(n): P=3(n+3)^{-1}$, where $n$ is defined by a proposed overall reaction (3.1) at the oxide/ electrolyte interface.

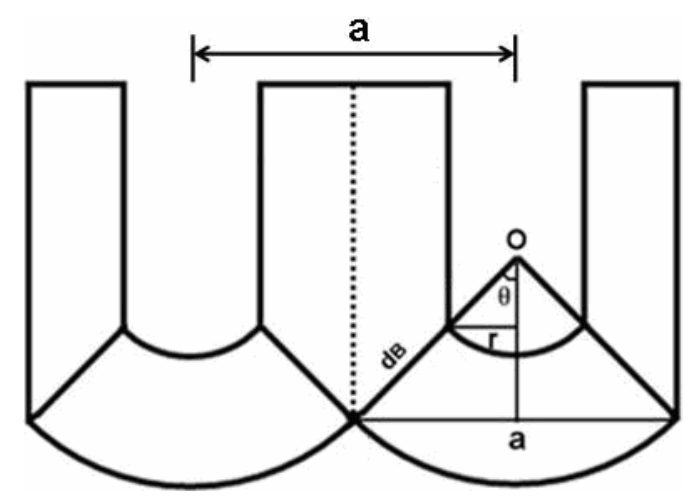

Figure 3-13. Schematic diagrams showing the geometrical relation for the pore radius $(r)$, cell size $(a)$, and the barrier thickness $d_{B}$, of AAO nanopore arrays.

As seen from Figure 3-13, there exist fixed relationships between the dimension parameters of the pores of AAO films. $\sin \theta=\frac{\frac{1}{2} a-r}{d_{B}}, \quad p=\frac{2 \pi}{\sqrt{3}}\left(\frac{r}{a}\right)^{2}, \quad a=\left(\frac{2 \pi}{\sqrt{3} p}\right)^{1 / 2} r \quad, \quad$ and $r=d_{B} \sin \theta /\left(\frac{1}{2}\left(\frac{2 \pi}{\sqrt{3} p}\right)^{1 / 2}-1\right)$. For aluminium anodised in $0.3 \mathrm{M}$ oxalic acid at $15^{\circ} \mathrm{C}$, $P=21.3 \exp \left(-6.46 \times \frac{U}{d_{B}}\right) \cdot{ }^{225}$ If we accept the thickness of oxide layer is exponentially related to the formation voltage, the relation can be described as $d_{B}=d_{\text {final }}(1-\exp (-\gamma U))+d_{0}$, where $d_{0}=1.5$ $\mathrm{nm}, d_{\text {final }}=236.4 \mathrm{~nm}$ and $\gamma=5.6 \times 10^{-3} \mathrm{~V}^{-1} \cdot 225$ Pore diameter can be described as 


$$
\begin{aligned}
& d_{\text {pore }}=2 r=\frac{2 d_{B} \sin \theta}{\frac{1}{2}\left(\frac{2 \pi}{\sqrt{3} p}\right)^{1 / 2}-1} \\
& =\frac{4 \times[236.4(1-\exp (-0.0056 U))+1.5]}{\left(\frac{2 \pi}{\sqrt{3} \times 21.3 \exp \left(-6.46 \times \frac{U}{236.4(1-\exp (-0.0056 U))+1.5}\right)}\right)^{1 / 2}-2} \sin \theta
\end{aligned}
$$

If we assume the current density is exponentially related to the applied voltage, the thickness of the oxide layer: $d_{B}=411.7-\frac{3180.8}{7.726+0.0256 U},{ }^{225}$ the pore diameter

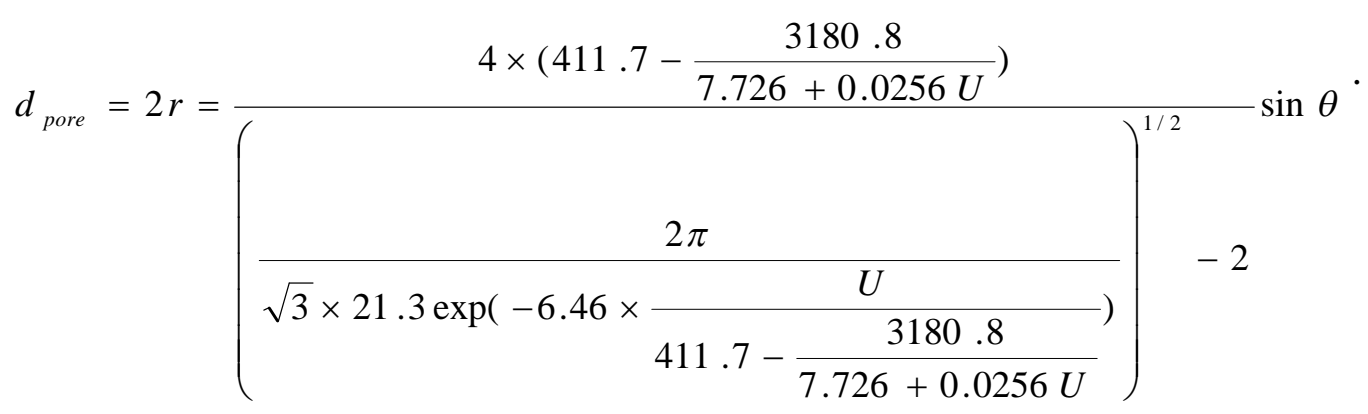

Since the value of $\sin \theta$ varies slightly with applied voltage as indicated experimentally, we are unable to give the relationship between the pore size of AAO and the applied voltage before the dependence of $\sin \theta$ on the applied voltage is obtained. Taking the common mild anodisation condition in $0.3 \mathrm{M}$ oxalic acid for instance, $a / U=2.5 \mathrm{~nm} \mathrm{~V}^{-1}, r / U=0.45 \mathrm{~nm} \mathrm{~V} \mathrm{~V}^{-1}$ and $d_{B} / U=1.3$ $\mathrm{nm} \mathrm{V} \mathrm{V}^{-1}$, as observed experimentally, ${ }^{70}$ the corresponding $\sin \theta=0.616$. Neglecting the slight change with voltage, $\sin \theta$ could be treated as a constant.

Then the plots of estimated AAO pore diameter versus the applied voltage are shown in Figure 3-14. From Figure 3-14a, a smallest value of pore diameter corresponds to a formation voltage of about $7 \mathrm{~V}$. This is in accordance with the discovery by Ono et al. ${ }^{237}$ In which the pore diameters of AAO films formed in four major electrolytes, sulfuric acid, oxalic acid, chromic acid and phosphoric acid, all show a smallest value at a formation voltage of about $5 \mathrm{~V}$ and became larger at both lower and higher voltages. Ono et $a l^{237}$ explained the pore diameters formed at lower voltages by the low efficiency of film formation at low current density ${ }^{238}$ and low electric field strength accompanied by low current density which leads to the decrease of cell wall 
thickness. ${ }^{239}$ It was assumed in general terms that the increase in pore diameters due to the electric field effect exceeds the decrease in pore diameters associated with the voltage decrease when the formation voltage is lower than $5 \mathrm{~V}$.

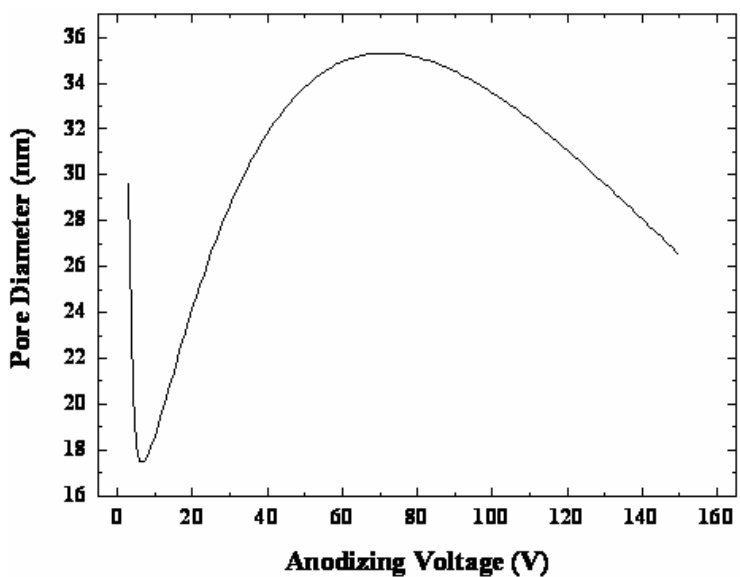

(a)

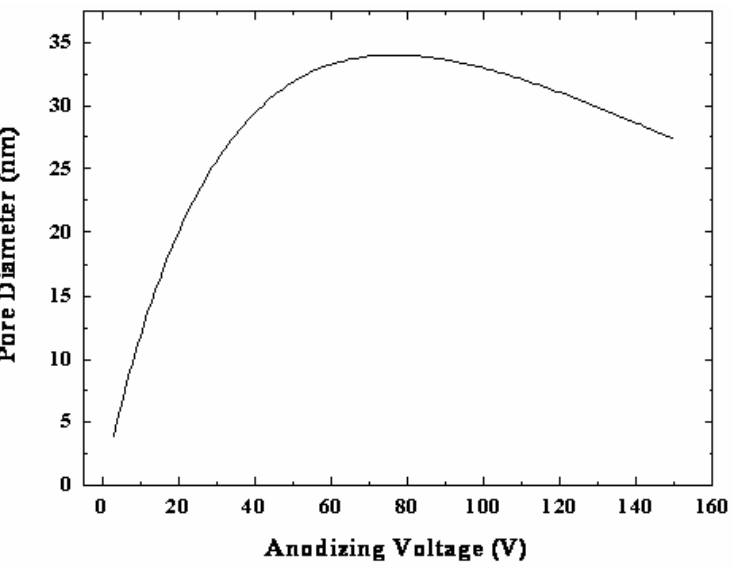

(b)

Figure 3-14. Plots of the estimated pore diameter versus the applied voltage for an AAO film anodised in $0.3 \mathrm{M}$ oxalic acid: (a) when barrier thickness has an exponential relation with voltage, (b) when current density has an exponential relation with voltage.

Below we explain the existence of smallest peak value of pore diameter at a formation voltage of about $7 \mathrm{~V}$ according to the proposed equifield strength model. In this model the porosity of the hexagonal porous AAO films $p=\frac{\pi}{2 \sqrt{3}}\left(\frac{d_{\text {pore }}}{a}\right)^{2}$, and the porosity increases exponentially with decreasing electric field strength and the formation voltage. For AAO prepared in $0.3 \mathrm{M}$ oxalic acid at $15{ }^{\circ} \mathrm{C}$, there is $P=21.3 \exp (-6.46 F)$, where the electric field strength $F=U / d_{B} . d_{\text {pore }} / a$ increases with decreasing voltage, $a=2 d_{B} \sin \theta+d_{\text {pore }}$, and, $d_{B}=d_{\text {final }}(1-\exp (-\gamma U))+d_{0}$ where $d_{0}=$ $1.5 \mathrm{~nm}, d_{\text {final }}=236.4 \mathrm{~nm}$ and $\gamma=5.6 \times 10^{-3} \mathrm{~V}^{-1}$. Neglecting the slight change of $\sin \theta$ with applied voltage, a smallest pore diameter at a formation voltage of about $7 \mathrm{~V}$ could be obtained via simple mathematical calculation as indicated by Figure 3-14a. Besides, there are also maximum values of the pore diameters at $71 \mathrm{~V}$ and $77 \mathrm{~V}$ in Figure 3-14a and 3-14b respectively, which need to be confirmed experimentally. 


\subsection{Local temperature effect on the dissolution rate}

Described by the field-assisted dissolution mechanism, when a high field is applied, the Al-O bond of alumina is lengthened and weakened due to strong polarization, it will be much more easily for the $\mathrm{H}^{+}$ions in the electrolyte to react with and dissolve it. On the other hand, the oxidation rate of the aluminium is determined by the current density of the anodisation. According to the high field conductivity theory: for valve metals, the current density $(j)$ can be related to the potential drop $(U)$ across the barrier layer of thickness $t_{\mathrm{b}}: j=j_{0} \exp \left(\beta U / t_{\mathrm{b}}\right)$, where $j_{0}$ and $\beta$ are material-dependent constants at a given temperature and $U / t_{\mathrm{b}}$ is the effective electric field strength $(E)$ across the barrier layer. In a certain electrolyte and applied voltage, the current density and the oxidation rate should be determined by the thickness of the barrier layer.

The oxidation rate of AAO is only determined by the current density, and the dissolution rate of the oxide mainly depends on the local temperature and the effective electric field strength in a certain electrolyte. Considering the equilibrium of the dissolution and oxidation rates, the dissolution rate should be also determined by the current density. That means the dissolution rate of the oxide is determined by the electric field strength across the barrier layer and the current induced joule heating effect. When the current density elevates, the increased oxidation rate should be compensated by the increasing dissolution rate by the contribution of electric field strength and the local temperature rising.

Here we conceive that the local temperature rising not only enhances the chemical dissolution rate but more importantly the field-assisted dissolution rate of the oxide. When a high field is present, the Al-O bond of the alumina is lengthened and weakened, it will be more easily for the $\mathrm{H}^{+}$ions in the electrolyte to react with and dissolve it. Local temperature rising can help to increase the effective local $\mathrm{H}^{+}$ion concentration near the dissolution interface, i.e. increasing the diffusion rate. There will be more hydrolyzed $\mathrm{H}^{+}$ions to reach the barrier and electrolyte interface and react with the oxide in a unit time, consequently increasing the dissolution rate of the oxide.

In addition, the increased electric field strength at the outer surface of the barrier layer may cause more severe lengthening and weakening of the Al-O bond. Based on these considerations, the temperature and electric field strengthened field-assisted dissolution mechanism as discussed above should be account for the increasing dissolution rate at elevated current density. 


\subsection{The $\mathrm{nm} / \mathrm{v}$ ratio for the barrier layer}

The thickness of the barrier layer plays an important role in the anodic formation of aluminium oxide, as it is established above that the requirement of uniform electric-field distribution, i.e. constant thickness barrier layer at the entire barrier/metal interface is the main internal driving force for the self-organisation of hexagonal pattern. In the following section we try to study the impact factors on the thickness of the barrier layer.

As it is known that the effective electric field strength across the barrier layer determines the incorporation rate of the oxygen-containing anions which is equal to the dissolution rate of barrier layer at the electrolyte/oxide interface, and the dissolution rate of the barrier layer can be increased by decreasing the $\mathrm{pH}$ value of the electrolyte or elevating the temperature. If the local temperature at the anode surface increases or the $\mathrm{pH}$ of the electrolyte decreases, leading to a faster dissolution rate, a higher electric field will be required to maintain the equilibrium between the dissolution and oxidation of the barrier layer. Hence, the effective electric field strength across the barrier layer, i.e., the value of $U / t=E$ in the produced anodic alumina should increase as $\mathrm{pH}$ of the electrolyte decreases or the local temperature at the dissolution interface rises. This coincides with the work by O'Sullivan et $a l^{25}$ shown in Table 3-1.

For AAO produced at a constant voltage, raising the temperature of the electrolyte will lead to an increase of the effective electric field strength across the barrier layer, i.e., a smaller $\mathrm{nm} / \mathrm{V}$ ratio for the barrier thickness. A higher concentration of the electrolyte corresponds to a lower $\mathrm{pH}$ value and faster dissolution rate of aluminium oxide. Therefore, the $\mathrm{nm} / \mathrm{V}$ ratio for the barrier thickness decreases with increasing concentration of the electrolyte.

In contrast, for $\mathrm{AAO}$ produced at a constant current density, the $\mathrm{nm} / \mathrm{V}$ ratio for the barrier thickness increases with increasing concentration of the electrolyte and the solution temperature. As discussed above, the current density can be divided into two parts: one from the dissolution of oxide $j_{\text {oxide }}$ and the other from the field enhanced dissociation of the water $j_{\text {water }}$. Increasing the concentration of the electrolyte or the temperature will increase the part of current density from the dissolution of oxide $j_{\text {oxide }}$. Since the total current density remain a constant, $j_{\text {water }}$ from the field enhanced dissociation of the water will decrease. Consequently, the effective electric field 
strength across the barrier layer should decrease and the $\mathrm{nm} / \mathrm{V}$ ratio of the barrier layer increases.

Previous work showed that for phosphoric acid, the cell dimension/voltage ratio was 2.77 $\mathrm{nm} / \mathrm{V}$ at $100 \mathrm{~V} ;{ }^{25}$ and $2.5 \mathrm{~nm} / \mathrm{V}$ at $195 \mathrm{~V}$; for oxalic acid, the ratio was $2.5 \mathrm{~nm} / \mathrm{V}$ at $40 \mathrm{~V}$ and 2 $\mathrm{nm} / \mathrm{V}$ at $140 \mathrm{~V} ;^{.70}$ and for the sulfuric acid, it was $2.5 \mathrm{~nm} / \mathrm{V}$ at $25 \mathrm{~V}$ and $1.8 \mathrm{~nm} / \mathrm{V}$ at $70 \mathrm{~V} .^{91} \mathrm{The}$ corresponding cell dimension/voltage ratio decreases fastest in the sulfuric acid and slowest in the phosphoric acid. As the barrier layer thickness/voltage ratio decreases with increasing current density, this difference could be attributed to the different increasing rate of the current density in the three electrolytes: sulfuric > oxalic > phosphorus..

It has been discussed above that higher current density and consequent increasing of the local temperature will cause a smaller $\mathrm{nm} / \mathrm{V}$ anodic ratio for the barrier layer. If the curvature $W$ of the barrier layer kept at the same value or changed slightly, the proportion for $t_{\text {barrier }} / t_{\text {wall }}$ will retain a constant value approximately. The pore diameter and the thickness of the wall will also have a decreased $\mathrm{nm} / \mathrm{V}$ ratio as the $t_{\text {barrier }}$, subsequently decreasing the cell dimension at a certain voltage. So the local heating induced by increasing current density has an impact on the cell dimension which could not be neglected especially for a rather high current density. As reported by Lee $e t a l,{ }^{70}$ for the anodic alumina prepared in $0.3 \mathrm{M}$ oxalic acid with an anodising voltage of $120 \mathrm{~V}$, the anodic ratio for the barrier layer is about $1 \mathrm{~nm} / \mathrm{V}$, compared to the typical $1.3 \mathrm{~nm} / \mathrm{V}$ ratio of the barrier layer thickness for AAO anodised at $40 \mathrm{~V}$. This could be ascribed to the local heating effect caused by much higher current density for hard anodisation.

Table 3-1. Variation of barrier layer $\mathrm{nmV}^{-1}$ ratio with acid concentration and temperature at constant current density or constant voltage. (By O'Sullivan et al, 1970) ${ }^{25}$

$\begin{array}{cccc}\begin{array}{c}\text { electrolyte } \\ \text { concentration/M }\end{array} & \text { temperature } /{ }^{\circ} \mathrm{C} & \begin{array}{c}\mathrm{nm} \mathrm{V}-1 \text { at constant } \\ \text { current density }\end{array} & \begin{array}{c}\mathrm{nm} \mathrm{V}-1 \text { at } \\ \text { constant voltage }\end{array} \\ 0.4 & 20 & 0.89 & 1.14 \\ 0.4 & 25 & 0.90 & 1.09 \\ 0.4 & 30 & 1.05 & 1.04 \\ 0.4 & 25 & 0.90 & 1.09 \\ 1.5 & 25 & 1.10 & 1.04 \\ 2.5 & 25 & 1.17 & 0.82\end{array}$




\section{Formation of Self-ordered ATO Nanotubular Arrays}

Although the fabrication of porous aluminium oxide layers via anodisation of aluminium has a long history, ${ }^{11}$ only in recent years was this process achieved for titanium. ${ }^{28}$ Since the formation of porous anodic oxide films could only be achieved in electrolytes in which the oxide could be dissolved slightly, usually fluoride containing electrolytes were chosen for the anodisation of titanium. Compared to the metal substrates, nanoarchitectured porous anodic titanium oxide films are expected to have specific functional properties, which may be promising in applications in dye-sensitized solar cells, ${ }^{23}$ photocleavage of water, ${ }^{22}$ photocatalyst, ${ }^{240}$ and in application in biology. $^{21}$

As shown in Figure 4-1, highly ordered anodic titanium oxide nanotube arrays could be achieved via anodisation at $60 \mathrm{~V}$ in ethylene glycol containing 0.3 wt $\% \mathrm{NH}_{4} \mathrm{~F}$ and 2 wt $\% \mathrm{H}_{2} \mathrm{O}$. While the anodising voltage drops, the mean diameter of the corresponding ATO nanotube cells decreases as well as the ordering of the nanotube arrays.

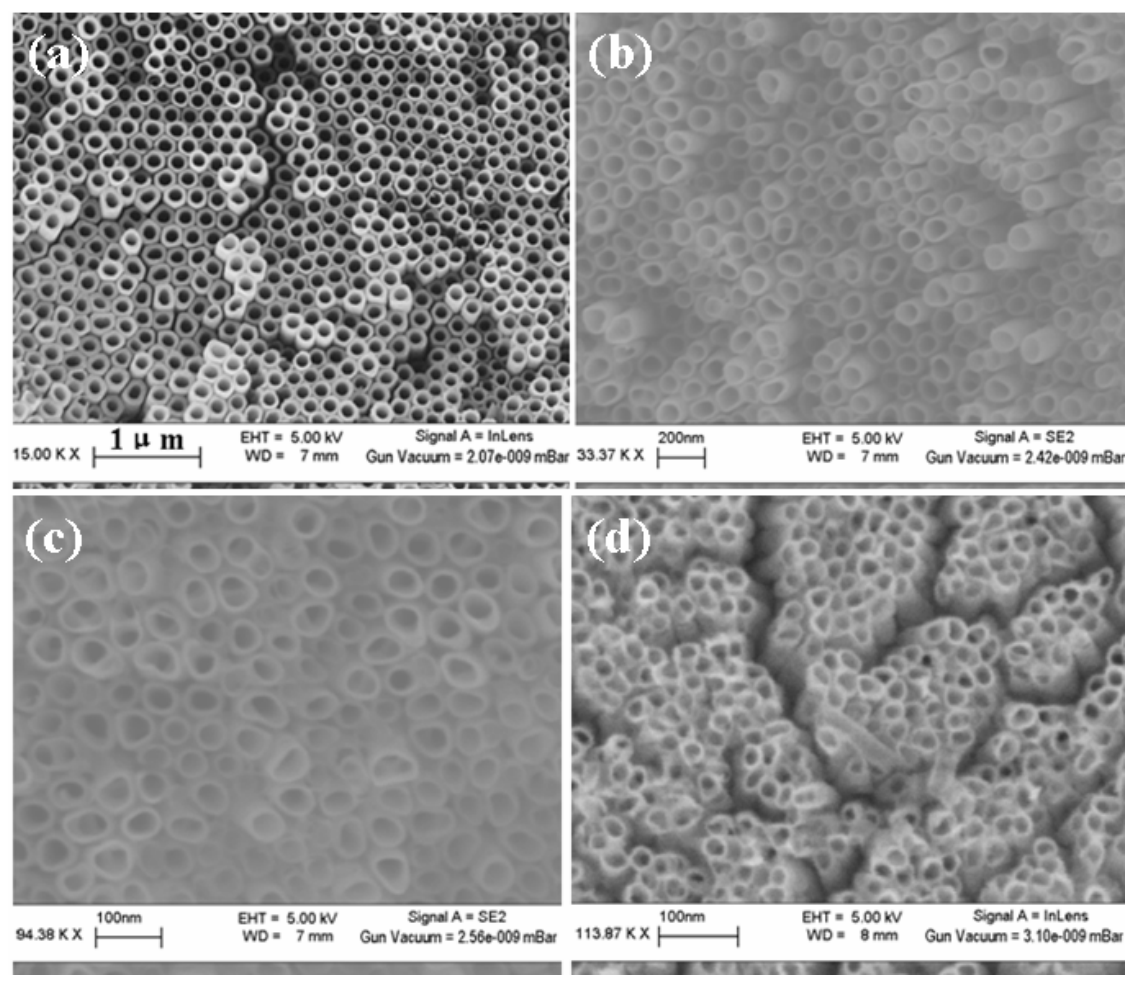

Figure 4-1. Morphologies of ATO films produced in ethylene glycol containing $0.3 \mathrm{wt} \% \mathrm{NH}_{4} \mathrm{~F}$ and 2 wt $\% \mathrm{H}_{2} \mathrm{O}$ under different voltages: $60 \mathrm{~V}(\mathrm{a}), 25 \mathrm{~V}$ (b), $12 \mathrm{~V}$ (c), and $5 \mathrm{~V}$ (d), respectively. 
The most significant difference between ATO and AAO is that the former contains separated nanotubes while the latter is a continuous film with a pore array (Figure 4-2). The mechanism of this difference has not been well established. The microstructures of ATO are obviously more complicated than those in AAO. Even for AAO, the formation mechanism is still not fully understood. A widely accepted model for the hexagonal ordering in AAO is based on mechanical stress associated with volume expansion during the oxidation of aluminium. ${ }^{27}$
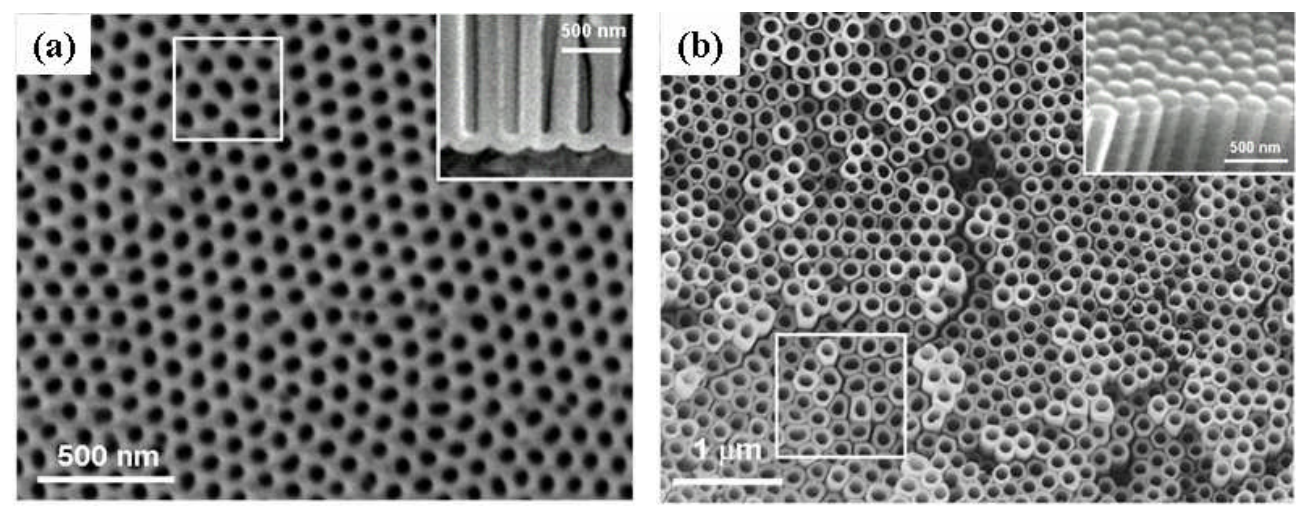

Figure 4-2. (a) Typical scanning electron microscopic (SEM) image (top view) of an AAO film prepared by two-step anodisation: an aluminium plate was anodised in $0.3 \mathrm{M}$ oxalic acid at $40 \mathrm{~V}$ for $3 \mathrm{~h}$, the porous oxide layer was removed by a mixed solution of chromic and phosphorus acid, and the plate was anodised again for $10 \mathrm{~h}$. The inset is a profile view of an AAO film prepared at $120 \mathrm{~V}$ in $2 \mathrm{wt} \% \mathrm{H}_{3} \mathrm{PO}_{4}$ aqueous solution for $60 \mathrm{~min}$. (b) SEM image of an ATO film prepared with the anodising voltage of $60 \mathrm{~V}$ and anodisation time of $16 \mathrm{~h}$. The inset shows the bottom of the film. Some regions with enlarged distorted pores are marked by a square.

However, it is difficult to use this model to elucidate the self-ordering process in ATO since the nanotubes are separated by at least a few nanometers. As discussed above, we have proposed an equifield strength model for explaining the formation of parallel pores and geometry of the pores in AAO. ${ }^{78,225}$ We believe this model can also be used in ATO and other porous metal oxides.

The equifield strength model has been used to elucidate the formation of the pores in ATO, the self-ordering and the geometry (e.g., hemispherical pore bottom) of the pores. TEM and SEM were applied to reveal the microstructures of ATO nanotubes, including double-layer wall and 
periodic O-ring like pattern on the outer surface of the nanotubes, therefore understanding the reason of the separation of the nanotubes. In addition, the porosity of ATO films was found to be governed by the relative dissociation rate of water which is dependent on anodisation conditions, such as electrolyte, applied voltage, current density, and electric field strength. With these achievements, the fabrication of ATO films can now be controlled more precisely.

\subsection{Microstructures of ATO}

With a long time of anodisation ( $15 \mathrm{~h}$ ), an ATO film with a thickness of about $40 \mu \mathrm{m}$ could be produced (Figure 4-3a). A top view on the opened ends of the nanotubes (Figure 4-2b) and a bottom view on the closed ends of the nanotubes (top part of Figure 4-3b) show that these nanotubes are almost hexagonally ordered. The morphology of the bottom of the nanotubes seems to be hemispherical. However, some distortions and non-uniform wall thicknesses of the nanotubes can be seen from the top-view images.
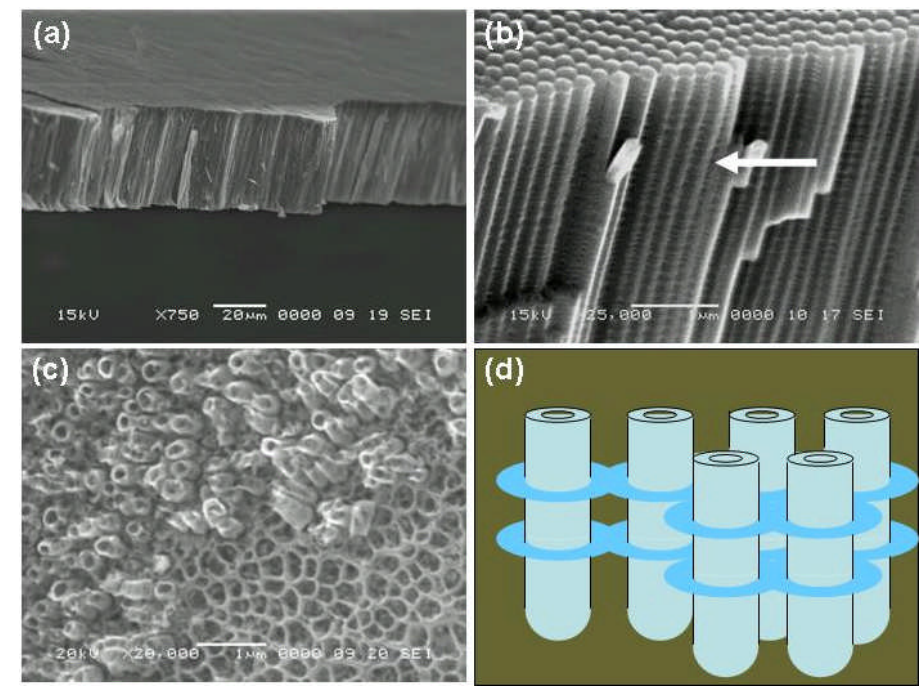

Figure 4-3. SEM images of the produced ATO films with (a) a profile view at a low magnification showing the film thickness, (b) a profile view at a larger magnification showing O-ring pattern as indicated by the arrow, (c) a top view of a film with nanotubes being partially removed. (d) Schematic drawing of the microstructure of ATO.

The enlarged SEM image along the profile direction shows the outer surface of the nanotubes 
with an O-ring like pattern. In fact, these O-rings are the remains of some two-dimensional sheets supporting the nanotubes. These porous sheets can be revealed when the nanotubes are partially removed by an ultrasonic treatment. To achieve this, a relatively thinner ATO film was prepared and the corresponding SEM image is shown in Figure 4-3c. When all the nanotubes were removed, the porous sheets can be collected. This discovery is important for future application of the materials. If these sheets can be maintained after crystallisation, they play a role of support to the nanotubes and can significantly increase the mechanical strength of the ATO films. The typical morphology of the as-prepared ATO film is like test-tubes stored in a tube stand as we often see on the laboratory benches (Figure 4-3d).
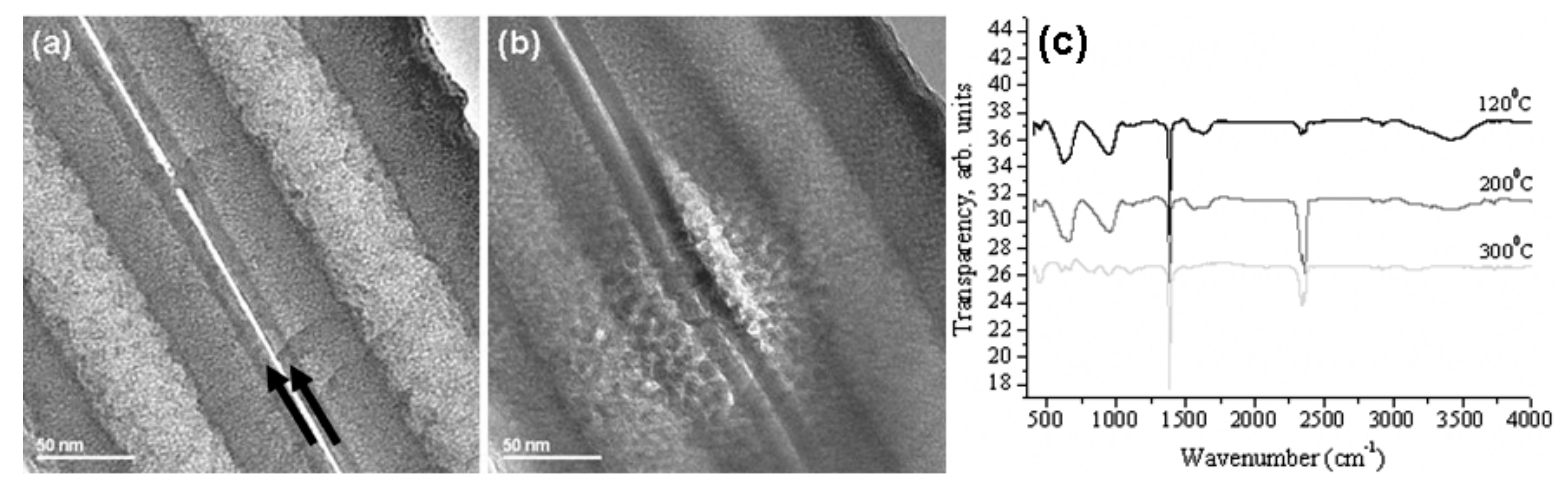

Figure 4-4. (a) TEM image of two nanotubes dropped from an ATO film, showing a double-layer wall. The arrows indicate the outer layers. (b) After electron beam irradiation for a few minutes, the inner layer and outer layer are separated. (c) Temperature dependent IR spectrum of ATO. The time for each annealing treatment is $2 \mathrm{~h}$.

Unlike AAO where the wall of the pores is monophasic aluminium oxide, the nanotubes in ATO have a double-layer wall as revealed by TEM images. Figure 4-4a is a TEM image of two parallel nanotubes $130 \mathrm{~nm}$ in diameter, $30 \mathrm{~nm}$ in thickness of the inner layer, $8 \mathrm{~nm}$ in thickness of the outer layer and with about $3 \mathrm{~nm}$ space between the nanotubes. After electron beam irradiation for a few minutes, the outer layer became separated from the inner layer due to the different thermal expansion coefficients (Figure 4-4b), confirming that these two layers have different compositions and an obvious boundary. Infrared spectrum of as-prepared ATO (Figure 4-4c) shows a peak at $\sim 1630 \mathrm{~cm}^{-1}$ and a broad band between $3000 \mathrm{~cm}^{-1}$ and $3700 \mathrm{~cm}^{-1}$, both 
corresponding to structural $\mathrm{OH}^{-} \cdot{ }^{78,241}$ The intensities of these peaks drop when the sample was annealed at higher temperature. Bearing in mind that Taveira $e t a l^{242}$ identified the existence of $\mathrm{Ti}(\mathrm{OH})_{4}$ by XPS in the compact layer formed at the early stage of anodisation of titanium, the infrared information together with the volume shrinkage behavior indicate that the outer layer is more likely to be some type of titanium hydroxide with a relatively lower density, while the inner layer is titanium oxide.

\subsection{Chemical reactions}

Chemical reactions during the anodisation of titanium are complicated and have been investigated by several groups. ${ }^{29,30,243}$ It is commonly accepted that the process includes field assisted oxidation of $\mathrm{Ti}$ metal to form $\mathrm{TiO}_{2}$, field assisted dissolution of $\mathrm{Ti}$ metal ions in the electrolyte, and chemical dissolution of $\mathrm{Ti}$ and $\mathrm{TiO}_{2}$ due to etching by fluoride ions. ${ }^{18}$ Unfortunately, no quantitative investigation was reported and the role of the dissociation of water is often ignored. We try to propose more detailed reactions based on our microstructural investigation.

When titanium is anodised, a barrier layer of titanium oxide forms on the metal surface. The initiation of pore formation should be the same as that in AAO, which is due to defects and a rough surface in the barrier layer. ${ }^{48}$ We now consider chemical reactions when a pore is developed from a surface pit. At the electrolyte/oxide interface, titanium oxide is dissolved in the fluoride anion containing electrolyte. This process will reduce the thickness of the oxide layer. Suppose all the oxide anions created from this dissolution migrate from the electrolyte/oxide interface to the oxide/ $\mathrm{Ti}$ interface to form $\mathrm{Ti}$ oxide or $\mathrm{Ti}$ hydroxide, the amount of oxide anions is just enough to form a new layer at the pore bottom and the thickness of the oxide layer in the hemispherical bottom is maintained. On the other hand, a large amount of oxide anions are still needed to build the wall of the pores with a volume corresponding to $\Delta L$ during the anodisation time $\Delta t$ (Figure 4-5a). 

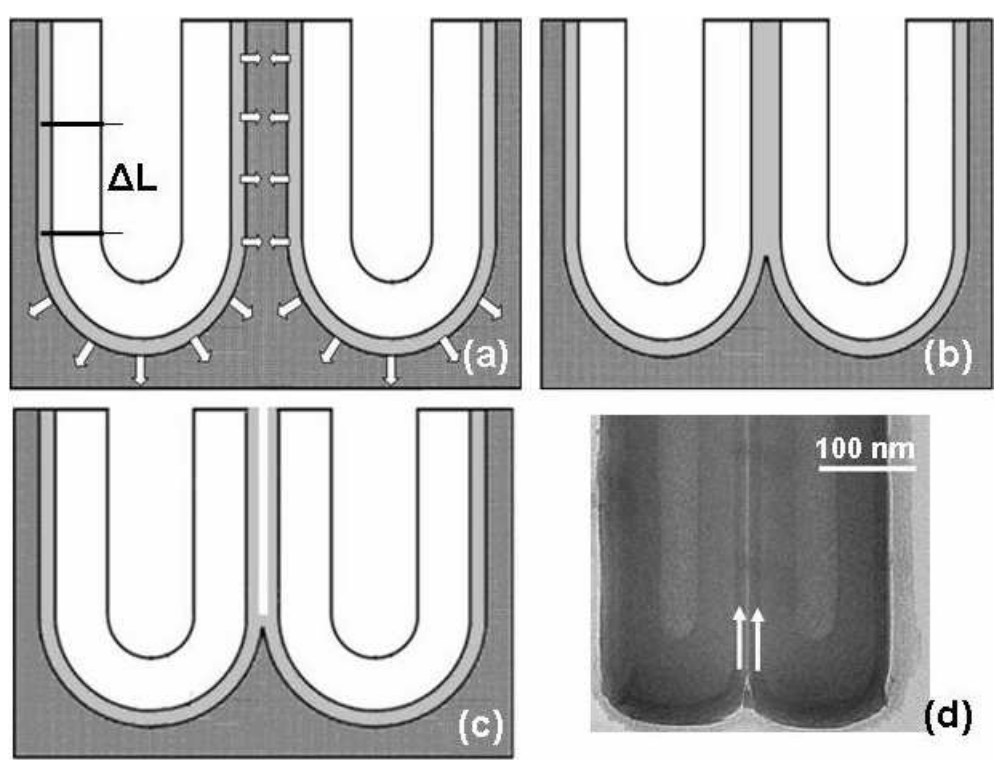

Figure 4-5. Schematic drawing of nanotube formation in ATO. (a) Two neighboring nanotubes with Ti metal in between would move closer to each other by expanding their diameter. (b) The expansion stops when they touch each other. (c) The hydroxide layer in between two nanotubes shrinks along the side surfaces when it decomposes. (d) The corresponding TEM image of such twin nanotubes.

These oxide anions are from the dissociation of water on the oxide surface. Consequently, the overall reaction at the electrolyte/oxide interface can be written as

$\mathrm{TiO}_{2}+n \mathrm{H}_{2} \mathrm{O}+6 \mathrm{~F}^{-} \rightarrow\left[\mathrm{TiF}_{6}\right]^{2-}+(n+2-x) \mathrm{O}^{2-}+x \mathrm{OH}^{-}+(2 n-x) \mathrm{H}^{+}$

where $n$ is introduced to indicate the ratio of dissociation of water and dissolution of $\mathrm{TiO}_{2}$ and this ratio is the key factor in governing the porosity of the ATO films as we discuss later. The protons in the form of $\mathrm{H}_{3} \mathrm{O}^{+}$move towards the cathode of the electrochemical cell, $\left[\mathrm{TiF}_{6}\right]^{2-}$ anions are dissolved into the electrolyte, while the oxide anions migrate in the electric field from the solid surface to the hydroxide/metal interface, contributing to the formation of the oxide/hydroxide layer. The hydroxide at the interface of oxide/hydroxide interface decomposes by losing protons continuously to form titanium oxide. The thicknesses of both the oxide and hydroxide layers are constant under certain anodisation conditions in a steady state. The overall oxidation reaction can be expressed as: 
$2 \mathrm{Ti}+\mathrm{O}^{2-}+4 \mathrm{OH}^{-} \rightarrow \mathrm{TiO}_{2}+\mathrm{Ti}(\mathrm{OH})_{4}+6 \mathrm{e}^{-}$

Reaction (4.2) leads to an increase of the thickness of the oxide layer. When titanium is oxidized into $\mathrm{Ti}^{4+}$ cations, part of which stay in the oxide/hydroxide layer and other part move directly from the hydroxide/metal interface towards the electrolyte without forming oxide or hydroxide.

\subsection{Equifield strength requirement and the ATO formation mechanism}

When a constant voltage $U$ is applied to the oxide layer, the electric-field strength $E$ in the oxide layer is inversely proportional to the oxide layer thickness $d, E=U / d$. When titanium is anodised in fluorine-containing electrolyte, the dissolution rate of $\mathrm{TiO}_{2}$ is fast, resulting in a reduction of thickness $(d)$ of the oxide layer and therefore an increase of the field strength. The dissociation rate of water will then be increased and the growth of oxide layer be enhanced. Finally, an equilibrium state between the oxidation and the dissolution processes will be approached with a constant barrier thickness $\left(d_{\mathrm{B}}\right)$ corresponding to a constant field strength $\left(E_{B}\right)$ in the whole anodisation area.

Since the whole electrolyte/oxide interface has a uniform potential, so does the hydroxide/metal interface, the field direction is always perpendicular to the interfaces. Hemispherical morphology of the bottoms of the ATO nanotubes is the only shape which can meet the above mentioned equifield strength requirement. On the other hand, it was previously reported that the hemispherical pore bottom can not be achieved when a very strong $\mathrm{Cl}^{-}$anions containing acidic solution is used in anodisation of titanium, when chemical etch dominates the process and no stable oxide layer forms. ${ }^{244}$ In this case, the equifield strength model cannot be applied and a square shape or other non-spherical shape could appear. A HF-based electrolyte is also a too strong acid for anodisation of titanium and chemical etching is so significant that the nanotubes formed at earlier stage would be dissolved during the process and it is difficult to increase the thickness of the nanotubular arrays. ${ }^{28}$ This is the reason why people recently replaced HF-based electrolyte by $\mathrm{NH}_{4} \mathrm{~F}$-based electrolyte ${ }^{29}$ or a non-aqueous organic electrolyte. $^{30}$ 
Another important characteristic of the equifield strength model is that a single nanotube can not only grow at the bottom (downwards) but also expand its pore diameter as indicated by the arrows in Figure 4-5a. Only when two nanotubes touch each other as shown in Figure 4-5b, the expansion stops. The hydroxide layer can shrink along the directions perpendicular to the side surfaces of the nanotubes, forming separated nanotubes with double layer walls (Figure 4-5c). In this case, the bottoms of the nanotubes are still connected each other. The experimental observation for this microstructure is shown in Figure 4-5d.

Since the thickness of the wall is determined by the anodisation conditions, mainly the field strength, and the porosity of the ATO film is governed by the relative dissociation rate of water as we discuss later, the diameter of the nanotubes tends to be constant. The movement of the nanotube walls towards each other driven by the self enlargement potential eventually results in a shift of the nanotubes. This is the principle driving force of the self-organisation of the nanotubes in ATO to form a honeycomb pattern.

According to Grimes' group, ${ }^{129}$ the key processes responsible for anodic formation of titania should be the same as anodic alumina: (1) Oxide growth at the surface of the metal occurs due to interaction of the metal with $\mathrm{O}^{2-}$ or $\mathrm{OH}^{-}$ions. After the formation of an initial oxide layer, these anions migrate through the oxide layer reaching the metal/oxide interface where they react with the metal. (2) Metal ion $\left(\mathrm{Ti}^{4+}\right)$ migration from the metal at the metal/oxide interface. (3) Field assisted dissolution of the oxide at the oxide/electrolyte interface. ${ }^{25}$ Macak et $a l^{29}$ suggested that chemical oxide dissolution in ATO growth was a dominant factor rather than the .electric-field-aided ion transport. As the dissolution rate of titanium oxide greatly depends on the local acidification in fluoride containing electrolyte, the anodic pores grow at the higher acidification at the pore bottom than the pore mouth.

Both of the above processes cannot explain the formation of the gaps between the titania nanotubes. During the formation of porous anodic $\mathrm{TiO}_{2}$ nanotubes, $\mathrm{Ti}$ was found to be oxidized into hydroxide first, which was then decomposed to $\mathrm{TiO}_{2}$. Therefore, a double layer of $\mathrm{TiO}_{2}$ and $\mathrm{Ti}(\mathrm{OH})_{\mathrm{x}}$, can be observed. ${ }^{240}$ An aluminium hydroxide layer is never observed in AAO, since even if it forms, it would decompose quickly due to its very low decomposition energy. Unlike aluminium hydroxide, which has a low dissociation energy, e.g. $\Delta \mathrm{E}=3.80 \mathrm{kcal} / \mathrm{mol}^{245}$ from $\mathrm{Al}(\mathrm{OH})_{3}$ to $\mathrm{Al}_{2} \mathrm{O}_{3}$, the dehydration of $\mathrm{Ti}(\mathrm{OH})_{4}$ to $\mathrm{TiO}_{2}$ requires energy of up to $118 \mathrm{kcal} / \mathrm{mol}^{246}$ 
When individual nanotubes were examined by using TEM, it was found that the wall of the nanotubes consists of two layers. The inner layer was titania and the outer layer was titanium hydroxide, implying that $\mathrm{OH}^{-}$anions can move from the electrolyte/oxide interface to the oxide/metal interface to form a titanium hydroxide layer, although the exact formula could not be determined. It was assumed that the hydroxide layer decomposed continuously into oxide during the anodisation since its thickness at the nanotube bottom remains constant, resulting a volume shrinkage of the tube wall. That's the difference between the formation processes of AAO nanopore arrays and the ATO nanotubular arrays.
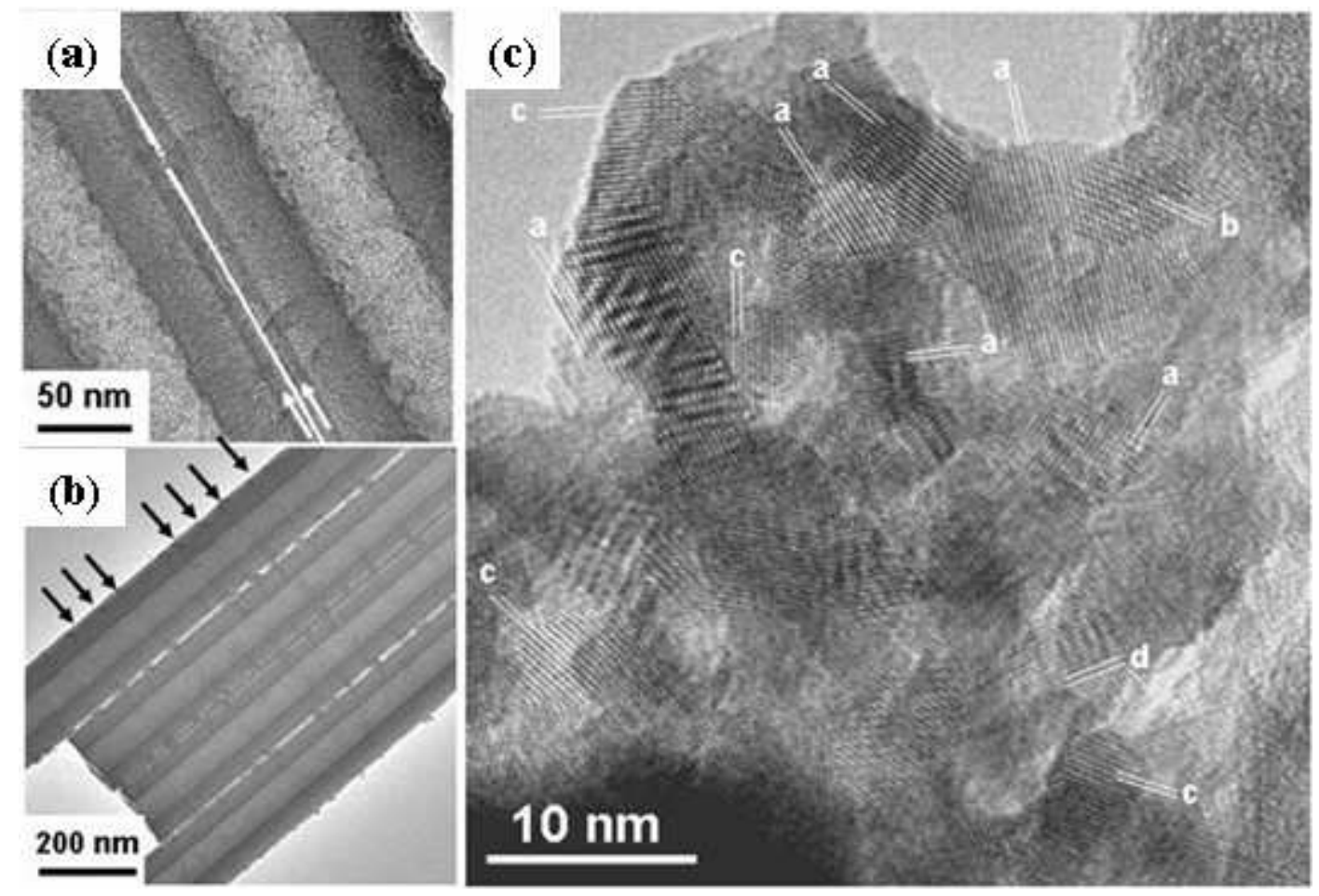

Figure 4-6. TEM images of (a) lower part and (B) upper part of ATO nanotubes, showing outer layers of titanium hydroxide indicated by two arrows (A), which decomposes into clusters of titania nanocrystallites (b). (c) HRTEM image of a typical cluster. The measured average d-spacings and corresponding crystal planes of $\beta-\mathrm{TiO}_{2}$ are (a) $0.310 \mathrm{~nm}$ (002), (b) $0.374 \mathrm{~nm}$ (201), (c) $0.302 \mathrm{~nm}(\overline{4} 01),(\mathrm{d}) 0.321 \mathrm{~nm}(\overline{1} 11)$.

As shown in Figure 4-6b, in the upper parts of nanotubes, the hydroxide layer has been replaced by some clusters of titania nanocrystallites. It is surprising to see that these clusters are 
located at certain positions in an ordered manner along the tube axis, appearing as some O-ring like patterns. These patterns have been often observed by many other groups in the SEM images and been explained as a variation of the nanotube diameter, ${ }^{29}$ which is incorrect according to the present work. HRTEM images further confirmed the structure of the clusters as seen in Figure 4-6c. Many d-spacings have been measured from the image and can be indexed to monoclinic $\beta-\mathrm{TiO}_{2}$ with $\mathrm{a}=1.216, \mathrm{~b}=0.374, \mathrm{c}=0.651 \mathrm{~nm}$ and $\beta=107.29^{247}$ (PDF No. 01-071-5576). These d-spacings cannot be indexed to other common $\mathrm{TiO}_{2}$ phases, such as brookite, rutile and anatase. It was noted that the $\beta-\mathrm{TiO}_{2}$ phase can be obtained from dehydration of titanium oxide hydroxide $\left(\mathrm{H}_{2} \mathrm{Ti}_{3} \mathrm{O}_{7}\right){ }^{248}$

Based on the above observations, we propose a new formation mechanism for ATO as shown in Figure 4-5a to c. $\mathrm{O}^{2-}$ and $\mathrm{OH}^{-}$anions migrate from the the $\mathrm{TiO}_{2} /$ electrolyte interface to the $\mathrm{TiO}_{2} / \mathrm{Ti}$ interface to oxidize $\mathrm{Ti}$, forming a layer of titanium hydroxide. While the oxidation process continues at the hydroxide/metal interface, the hydroxide at the oxide/hydroxide interface dehydrates to $\mathrm{TiO}_{2}$. In the steady state, the thicknesses of the oxide and hydroxide layers are constant, e.g., 33 and $8 \mathrm{~nm}$ respectively measured from Figure 4-6a. It is noted that dehydration of titanium hydroxide will result in higher density $\mathrm{TiO}_{2}$ and may create some local holes. When the hydroxide layer between the walls is partially dehydrated, the layer is divided into two by a gap as seen in Figure 4-6a. Further dehydration results in the formation of clusters of titania nanocrystallites (Figure 4-6c).

\subsection{Formation of O-rings}

The O-rings on the outer surface of the nanotubes are actually part of two-dimensional porous sheets as shown by the SEM images in Figure 4-3c. TEM images from multi-tube clusters and separated nanotubes also show this characteristic (Figure 4-7a,b). It is obvious that the inter-O-ring spacing is almost constant as seen in Figure 4-7b. Macak et $a l^{29}$ attributed the formation of the O-rings to a variation of nanotube diameter. It was reported that relatively regular current oscillations occur during anodisation. When the frequency of the current oscillations was converted into a length scale, it compared well to the distance between ridges on the side walls of the nanotubes. Thus, the current transients are correlated with the variations in 
the wall thickness. This can be explained by the fact that every current transient is accompanied by a $\mathrm{pH}$ burst at the pore tip. It seems that this explanation could well elucidate the formation of O-ring structures. However this was not in agreement with our TEM observation, since there is no variation of the nanotube diameters as observed from inner surface of the nanotubes, and the O-rings are extra parts connected only to the outer surface of the nanotubes.

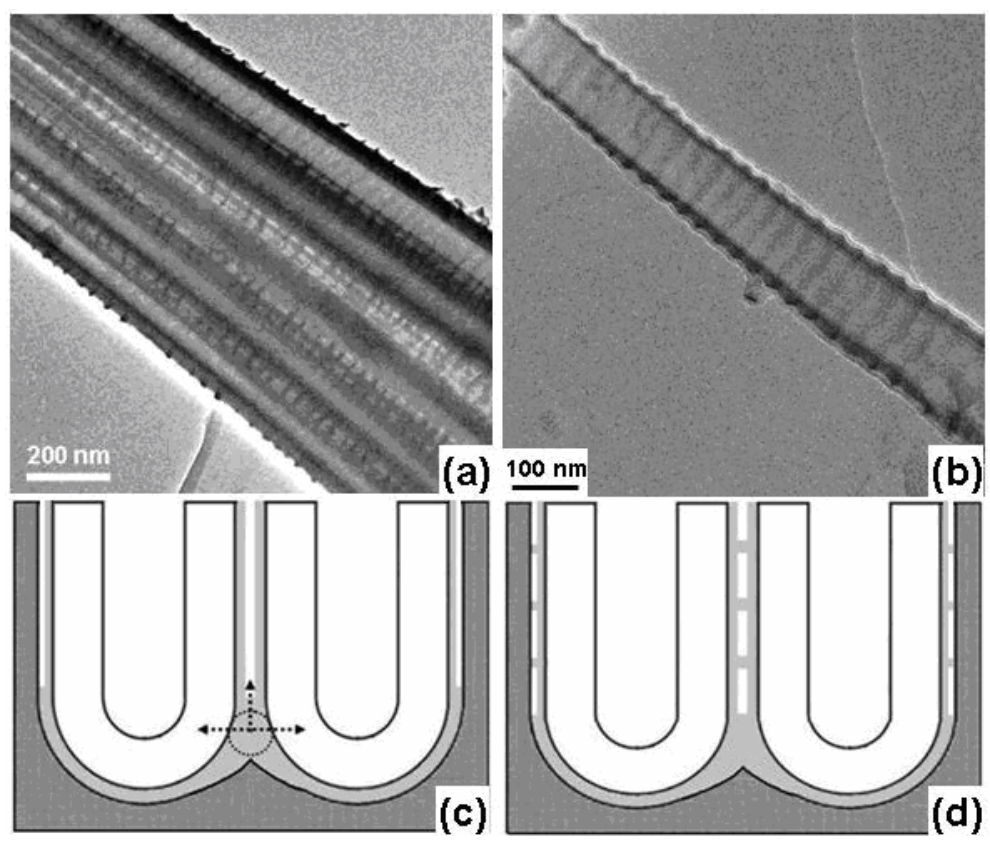

Figure 4-7. TEM images of (a) a bundle of ATO nanotubes and (b) a single nanotube, showing an O-ring pattern on the outer surface of the nanotubes. (c) and (d) Schematic drawings of the O-ring formation in ATO films. Arrows in (c) indicate the directions of volume contraction of the hydroxide layer.

Since the hydroxide layer is now revealed, the formation mechanism of these O-rings can be understood by considering a directional volume contraction. Due to the electric field and local heating enhanced dehydration, the ATO nanotubes could separate from each other as elucidated in Figure 4-5, where the direction of volume contraction of the hydroxide layer are normal to the walls. However, the direction of the field induced contraction can also be parallel to the growth direction of the nanotubes (field direction), leaving some small bridges of more condensed oxide in between nanotubes. As shown in Figure 4-7c, the electric field at the pore base between two 
neighboring tubes could be divided into the parallel and normal directions, leading to a volume contraction along and perpendicular to the wall. It is expected that the intervals of the O-rings (bridges), like the thickness of the barrier layer, is also a function of the applied voltage. For example, an increase of the spacing of the O-rings was observed, from $\sim 36.2 \mathrm{~nm}$ at $25 \mathrm{~V}$ to $~$ $62.0 \mathrm{~nm}$ at $60 \mathrm{~V}$ as measured from the TEM images, and the corresponding barrier thickness were $\sim 40.0 \mathrm{~nm}$ and $70.2 \mathrm{~nm}$, respectively.

\subsection{Porosity of anodic titanium oxide}

As with the formation of AAO, in the formation process of ATO, we assume all the oxide anions from the dissolution of titanium oxide will contribute to the oxidation of titanium at the bottom of the film, and all the oxide anions needed for building the wall, corresponding to a net change of $\Delta L$ during anodisation time of $\Delta t$, are from dissociation of water (Figure 4-8a). The total moles $\left(N_{o}\right)$ of the oxide anions from water dissociation during the time of $\Delta t$ are those in the volume of the newly formed part of the wall corresponding to $\Delta L$,

$$
N_{o}=\left(S_{C}-S_{P}\right) \times \Delta L \times D_{O}
$$

where $D_{o}$ is the mole density of oxygen anions in titanium oxide, $S_{c}$ is area of a cell containing one pore and $S_{p}$ is the pore area as shown in Figure $4-8 \mathrm{~b}$. To simplify the calculation, here we assume the mole density in the hydroxide layer is the same as that in the oxide layer and there is no gap between the nanotubes.

On the other hand, the total moles of $\mathrm{TiO}_{2}$ dissolving in the oxide/electrolyte interface following the eq. $(4.1)$ is $(1 / n) N_{0}$. The total moles of titanium dissolved during the time of $\Delta t$ is $S_{P} \times \Delta L \times D_{T i}$, where $D_{T i}$ is the mole density of $\mathrm{Ti}$ cations in $\mathrm{TiO}_{2}$. If all the oxygen-containing anions migrate across the oxide layer to contribute to the formation of $\mathrm{TiO}_{2}$, we have

$$
N_{O}=\left(S_{C}-S_{P}\right) \times \Delta L \times D_{O}=n \times S_{P} \times \Delta L \times D_{T i}
$$


where $D_{O}=2 D_{T i}$. Consequently, the porosity in the cell is

$$
\frac{S_{P}}{S_{C}}=\frac{2}{n+2}
$$
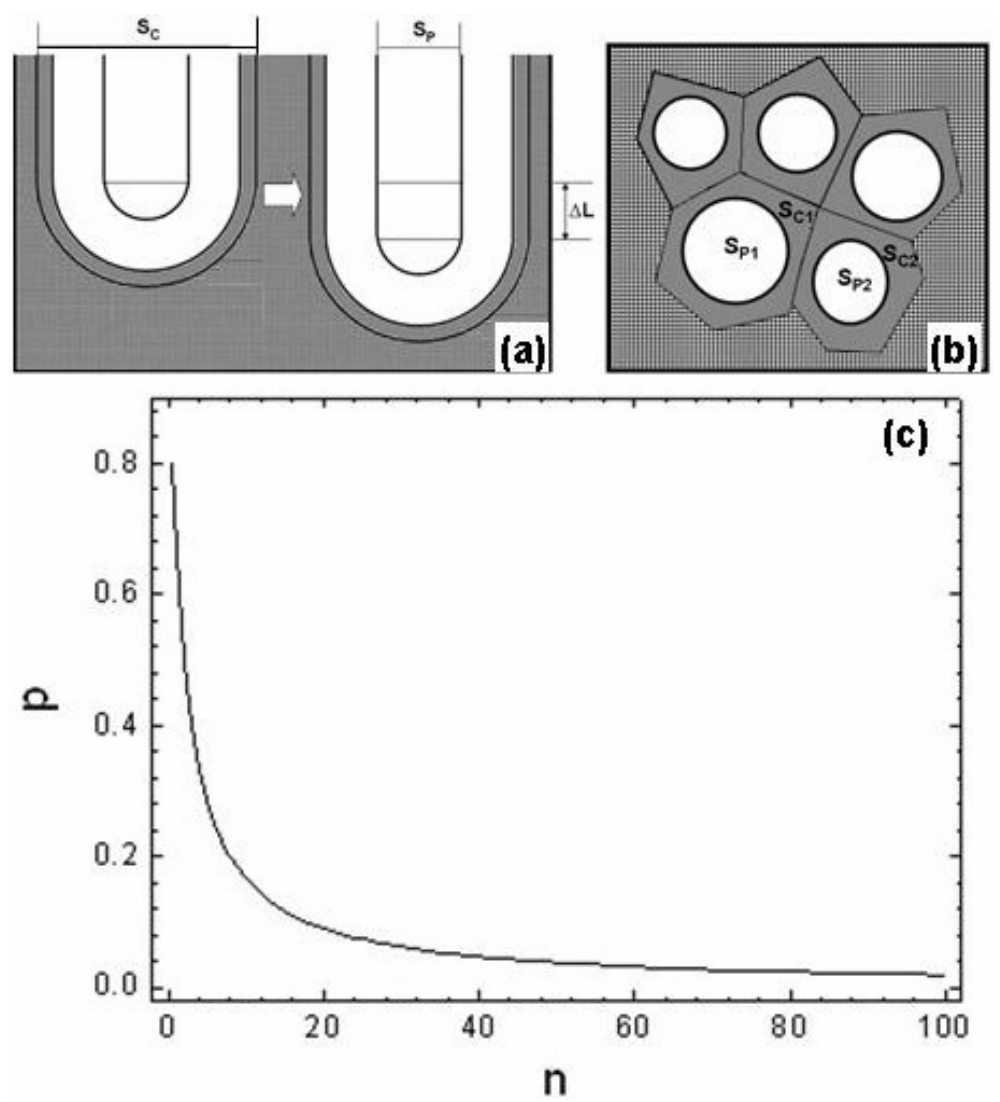

Figure 4-8. Schematic drawings of a single pore growth for a length increase of $\Delta L$ (a) and the compact pore array on top view (b). (c) Porosity of ATO $(P)$ as a function of the relative rate of water dissociation $(n)$ at the oxide/electrolyte interface.

Since the value of $n$ describes moles of water dissociated when one mole of $\mathrm{TiO}_{2}$ is dissolved, which should be constant at a certain anodisation condition, $n$ should be constant in all cells where the field strength across the barrier layer has a constant value. Therefore, the total porosity of the whole pore array is 


$$
P=\frac{S_{P t o t a l}}{S_{\text {Ctotal }}}=\frac{S_{P 1}}{S_{C 1}}=\frac{S_{P 2}}{S_{C 2}}=\frac{2}{n+2}
$$

The $P \sim n$ plot for ATO is shown in Figure 4-8c, which is similar to that for AAO. Directly measured from the TEM images of the ATO specimen prepared at $30 \mathrm{~V}$ in the present work, the ratio of the pore diameter to the cell diameter is about 0.348 . Assuming the film has a perfect hexagonal pore array, the porosity could be written as: $P=\frac{\pi}{2 \sqrt{3}}\left(\frac{D_{\text {pore }}}{D_{\text {Cell }}}\right)^{2}$. Thus the porosity of the $\mathrm{TiO}_{2}$ nanotubular array anodised under the given conditions is about $11.0 \%$ (Table 4-1), corresponding to $n=16.2$. It is necessary to point out that the porosity of ATO mentioned here could only be measured near the pore base as the severe chemical etching by the electrolyte could widen the pores significantly especially at the pore mouth.

The anionic current across the oxide layer can be divided into two parts, $j_{\text {oxide }}$ from the electric field enhanced dissolution of titanium oxide at the oxide/electrolyte interface and $j_{\text {water }}$ from the dissociation of water, i.e., $j=j_{\text {oxide }}+j_{\text {water }}$, where $j_{\text {water }} / j_{\text {oxide }}=n / 2$ derived from eq. (4.1), since the current density is proportional to the moles of anions created from the surface reactions. The porosity of the ATO film then has a relation with these current densities,

$$
P=\frac{2}{n+2}=\frac{j_{\text {oxide }}}{j_{\text {oxide }}+j_{\text {water }}}=\frac{j_{\text {oxide }}}{j}
$$

According to Tafel's Law, $j=j_{0} \exp (\beta U / d)=j_{0} \exp (\beta E)$, where $j_{0}$ and $\beta$ can be estimated from experiments. For example, when anodisation was carried out in ethylene glycol containing $0.3 \mathrm{wt}$ $\% \mathrm{NH}_{4} \mathrm{~F}$ and $2 \mathrm{wt} \% \mathrm{H}_{2} \mathrm{O}$, an empirical estimation of $j_{0}$ and $\beta$ could be derived by fitting the experimental data for the current density $(j)$ and effective field strength $(E)$ in Table $4-1$. Then we have $j_{0}=4.50 \times 10^{-4} \mathrm{~mA}$ and $\beta=10.09 \mathrm{~nm} / \mathrm{V}$, therefore,

$j=4.50 \times 10^{-4} \exp (10.09 E)$

In an analogy with the AAO case, the electric current contributed by dissolution of the 
barrier oxide at the pore base of ATO should have an exponential relation with the electric field strength. Therefore we can write $j_{\text {oxide }}=A \exp (k E)$, where $A$ is the pre-exponential factor for dissolution reactions and the coefficient $k$ depends on the working temperature and material property. Neglecting the current induced local heating of the barrier layer at the pore base, for fixed anodisation conditions, $A$ and $k$ can be treated as constants. To fit the experimental data for the current density from dissolution of the barrier layer $\left(j_{\text {oxide }}\right)$ and effective field strength $(E)$ in Table 4-1, the empirical relationship could be derived by

$j_{\text {oxide }}=2.50 \times 10^{-4} \exp (7.66 E)$

Table 4-1. Experimental data of anodisation of titanium in ethylene glycol containing $0.3 \mathrm{wt} \%$ $\mathrm{NH}_{4} \mathrm{~F}$ and $2 \mathrm{wt} \% \mathrm{H}_{2} \mathrm{O}$ : applied voltage $(U)$, measured current $(j)$, field strength $(E)$ and measured porosity $(P)$.

\begin{tabular}{llllll}
\hline No. & $U(\mathrm{~V})$ & $E\left(\mathrm{~V}^{-1} \mathrm{~nm}\right)$ & $j(\mathrm{~mA})$ & $P(\%)$ & $j_{\text {oxide }}(\mathrm{mA})^{\mathrm{a}}$ \\
\hline 1 & 10 & 0.565 & 0.15 & 15.5 & 0.023 \\
2 & 20 & 0.625 & 0.22 & 13.0 & 0.029 \\
3 & 30 & 0.667 & 0.37 & 11.0 & 0.041 \\
4 & 40 & 0.690 & 0.58 & 8.63 & 0.050 \\
5 & 60 & 0.855 & 2.50 & 6.96 & 0.174
\end{tabular}

${ }^{\mathrm{a}}$ Derived value from equation (4.7): $j_{\text {oxide }}=j \times P$

The porosity can be written as

$P=\frac{j_{\text {oxide }}}{j}=\frac{A \exp (k E)}{j_{0} \exp (\beta E)}=\frac{2.50 \times 10^{-4} \exp (7.66 \mathrm{E})}{4.50 \times 10^{-4} \exp (10.09 \mathrm{E})}=0.556 \exp (-2.43 E)$

From eqs. (4.8) and (4.10), the relationship between the porosity and the ionic current density $(j)$ can also be deduced as 


$$
P=0.556 \times\left(\frac{4.50 \times 10^{-4}}{j}\right)^{0.241}
$$

The corresponding $P \sim E$ and $P \sim j$ plots together with experimental data are shown in Figure 4-9, demonstrating a good matching between the experimental data and the calculated curves.

As the applied voltage is directly known from the experiments, the relationship between the porosity and the applied voltage is more useful in practice than that between the porosity and the field strength. In Tafel's Law, $j=j_{0} \exp (\beta U / d)$, where $U / d=E$ and $j$ is a function of both $U$ and $d$. We assume the current density increases exponentially with the applied voltage in a steady state in the working range for anodisation as implied by the observed data, then

$j=j_{0}{ }^{\prime} \exp (\alpha U)$

Used the current density and applied voltage listed in Table $4-1, j_{0}{ }^{\prime}=0.057 \mathrm{~mA}$ and $\alpha=$ $0.063 \mathrm{~V}^{-1}$.
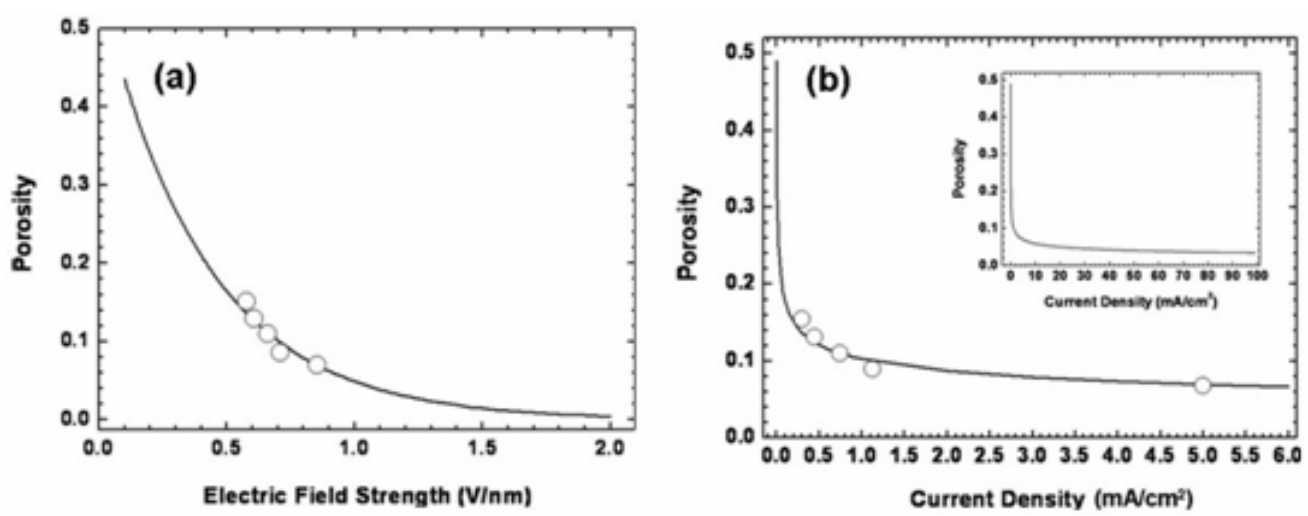

Figure 4-9. Porosity of ATO produced in ethylene glycol containing $0.3 \mathrm{wt} \% \mathrm{NH}_{4} \mathrm{~F}$ and $2 \mathrm{wt} \%$ $\mathrm{H}_{2} \mathrm{O}$ as a function of the electric field strength (a) and current density (b) across the oxide layer at the pore base. The inset of (b) shows $P \sim j$ plots in a larger range of current density. The solid curves are plotted via eqs. (4.9) and (4.10), while the circles represent the experimental data.

A combination of eqs. (4.8) and (4.12) enables us to derive a relationship between the 
thickness of the oxide layer and the applied voltage:

$d=\frac{\beta}{\alpha}\left(1-\frac{R}{R+\alpha U}\right)=121.6-\frac{588.8}{4.84+0.063 U}$

where $R=\ln \left(j_{0}, / j_{0}\right)$. It is noted that eq. (4.13) predicts a zero thickness of the barrier layer when no voltage is applied. The $P \sim U$ relation can then be established by a combination of eqs. (4.11) and (4.12):

$P=0.556 \times\left[\frac{7.89 \times 10^{-3}}{\exp (0.063 U)}\right]^{0.241}$

In another consideration, we know that the thickness of the barrier layer will be finite even at a very low voltage. We can model a relation between the thickness of the barrier layer and the voltage according to the following equation,

$d=d_{\max }(1-\exp (-\gamma U))+d_{0}$

where $d_{0}$ describes the thickness of the native barrier layer when no voltage is applied and is set to be $d_{0}=2.0 \mathrm{~nm}, d_{\max }$ is a measurement of the maximum thickness, and $\gamma$ describes the increase of the barrier thickness with $U$. Using the experimental data shown in Table 4-1, where the thickness of the barrier layer could be derived as $d=\mathrm{U} / \mathrm{E}$, we have $d_{\max }=103.9 \mathrm{~nm}$ and $\gamma=1.78 \times 10^{-2} \mathrm{~V}^{-1}$. Combination of eqs. (4.15) and (4.10) gives the relation between $P$ and $U$,

$P=0.556 \exp \left(-2.43 \times \frac{U}{d_{\text {final }}\left(1-\exp (-\gamma U)+d_{0}\right.}\right)$

Figs. 4-10a and b show the plots of porosity of ATO versus applied voltage when we assure either current density or barrier thickness has an exponential relation with the applied voltage respectively. A good agreement with the experimental results was observed in range of the 
working conditions for anodisation of titanium.
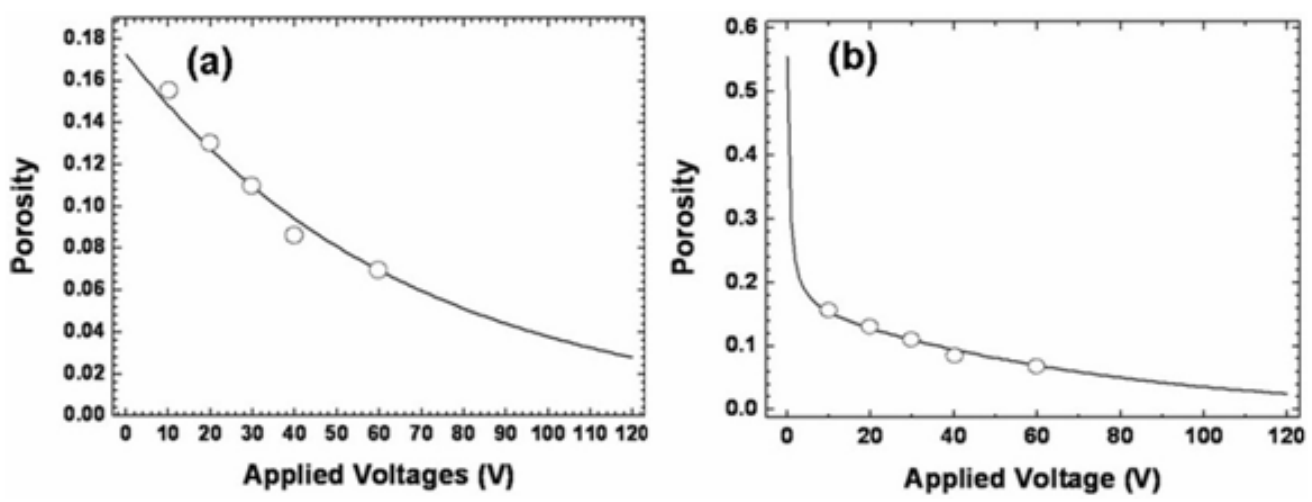

Figure 4-10. Porosity of ATO versus applied voltage in ethylene glycol containing 0.3 wt \% $\mathrm{NH}_{4} \mathrm{~F}$ and $2 \mathrm{wt} \% \mathrm{H}_{2} \mathrm{O}$ as a function of applied voltage, assuming (a) current density or (b) barrier thickness has an exponential relation with the applied voltage. The circles represent experimental data from the present work.

Eq. (4.13) predicts a zero thickness of oxide layer if no voltage is applied, which is not quite true as a thin native oxide layer could still form. The $P \sim U$ relation described in eq. (4.14) would encounter certain errors while fitting the experiments, which could be much more significant in the low voltage range. Taking this into account, we are much more confident with the exponential relationship of the barrier thickness with the applied voltage.

Since both the barrier layer at the pore bottom and the wall thickness are governed by the applied voltage, the established relationship of $P \sim U$ implies that the pore size in ATO is also governed by the applied voltage. Although a single nanotube will tend to increase its pore size according to the equifield strength model as we mentioned above, and experimental observation confirmed this mechanism, e.g. the diameter of the single nanotube in Figure 4-7b continuously increases from top to bottom, this self-adjustment can only be allowed over a small range since both the overall porosity and pore size are determined by the field strength. This is why uniform pore size can be achieved in a whole ATO film (Figure 4-11). When a single nanotube increases its pore size, beyond the limit determined by the porosity requirement, the nanotube may split into two or more nanotubes as shown in the inset of Figure 4-11. This phenomenon was also often observed from AAO films. 


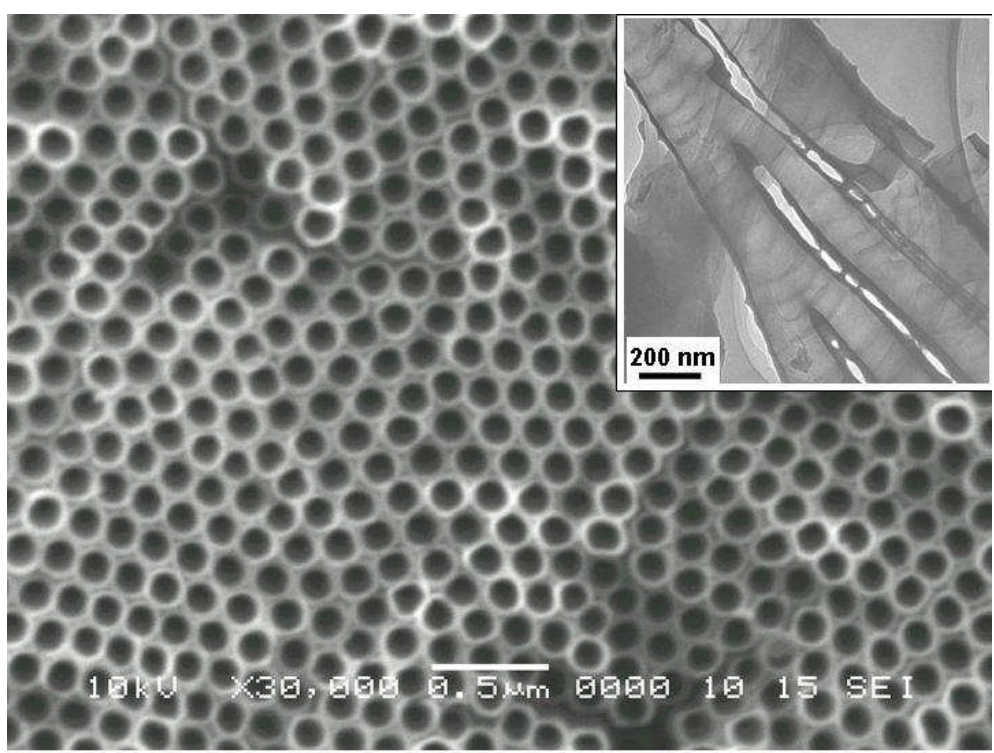

Figure 4-11. SEM image of a top view of an ATO film showing uniform pore size in a large area. The inset TEM image shows when the pore size of a single nanotube increases beyond the value restricted by the applied voltage where no neighboring nanotubes to stop its growth, it may split to two or more nanotubes.

\subsection{Pore size variation with voltage of ATO films}

In the anodisation of titanium, the overall reaction at the oxide/electrolyte interface can be written as eq. (4.1), and the corresponding $P \sim n$ relation is $P=2(n+2)^{-1}$. The pore diameter of ATO has a more simple relation with the cell size and the barrier thickness than AAO. As illustrated by Figure 4-12, the pore diameter of ATO with a hexagonal patterned tube array is $D_{p}$ $=2 r=2 \times\left(0.5 a-d_{B}\right)$, where $D_{p}$ is the diameter, $r$ the radii of pores, and $a$ the cell dimension.

Substituted in the porosity relation with the pore diameters and the cell dimension $P=\frac{2 \pi}{\sqrt{3}}\left(\frac{r}{a}\right)^{2}$, there is

$$
D_{p}=2 r=\frac{4 d_{B}}{\left(\frac{\sqrt{3}}{2 \pi} P\right)^{-0.5}-2}
$$




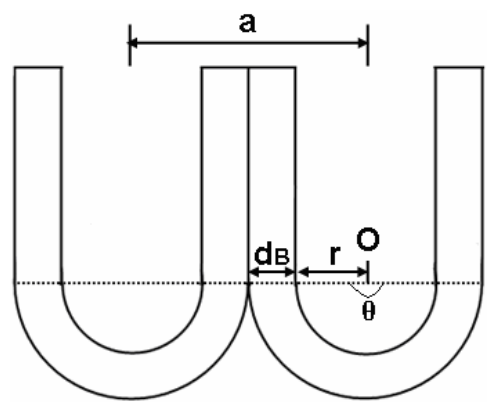

Figure 4-12. Schematic diagrams showing the geometrical relation for the pore radius $(r)$, cell size (a), and the barrier thickness $d_{B}$, in the case of ATO separated nanotubes.

If the thickness of the barrier layer $d_{B}$ took an empirical form $d_{B}=d_{\text {final }}(1-\exp (-\gamma U))+d_{0}$, where $d_{0}=2.0 \mathrm{~nm}, d_{\text {final }}=103.9 \mathrm{~nm}$ and $\gamma=1.78 \times 10^{-2} \mathrm{~V}^{-1},{ }^{1}$ The relation between the porosity $(P)$ and the applied voltage $(U)$ for ATO prepared under this condition should be: $p=0.556 \exp \left(-2.43 \times \frac{U}{d_{B}}\right) \cdot{ }^{249}$ Eq. $(4.17)$ can be transformed to:

$$
D_{p}=\frac{4 \times\left(d_{\text {final }}(1-\exp (-\gamma U))+d_{0}\right)}{\left[\frac{\sqrt{3}}{2 \pi} \times 0.556 \exp \left(-2.43 \times \frac{U}{d_{\text {final }}\left(1-\exp (-\gamma U)+d_{0}\right.}\right)\right]^{-0.5}-2}
$$

When assuming that the current density increases exponentially with the applied voltage, the relations between the porosity $(P)$, the barrier layer thickness $\left(d_{B}\right)$ and the applied voltage $(U)$ were found to be $P=0.556 \times\left[\frac{7.89 \times 10^{-3}}{\exp (0.063 U)}\right]^{0.241}$, and $d_{B}=121.6-\frac{588.8}{4.84+0.063 U}$, respectively. ${ }^{18}$ Eq. (4.17) can be transformed to

$$
D_{p}=\frac{4 \times\left(121.6-\frac{588.8}{4.84+0.063 U}\right)}{\left(\frac{\sqrt{3}}{2 \pi} \times 0.556 \times\left[\frac{7.89 \times 10^{-3}}{\exp (0.063 U)}\right]^{0.241}\right)^{-0.5}-2}
$$

In the present work, high purity titanium foil $(0.25 \mathrm{~mm}, 99.5 \%)$ was sonicated in acetone and then rinsed in deionized water. The anodisation was then performed in a home-made cell with 
typical conditions: anodisation voltages ranged from DC $10 \mathrm{~V}$ to $100 \mathrm{~V}$, the electrolytes used was 0.3 wt $\% \mathrm{NH}_{4} \mathrm{~F}$ in ethylene glycol with $2 \mathrm{wt} \%$ water, and the working temperature was at $15^{\circ} \mathrm{C}$. Measurement of the pore diameter of the produced ATO films was carried out using TEM on JEOL JEM-2011 electron microscope operated at $200 \mathrm{kV}$. To minimize the diameter widening effect caused by the strong chemical dissolution of the ATO films at the pore mouth, all the values of the pore diameters were measured at the pore base next to the metal/oxide interface.
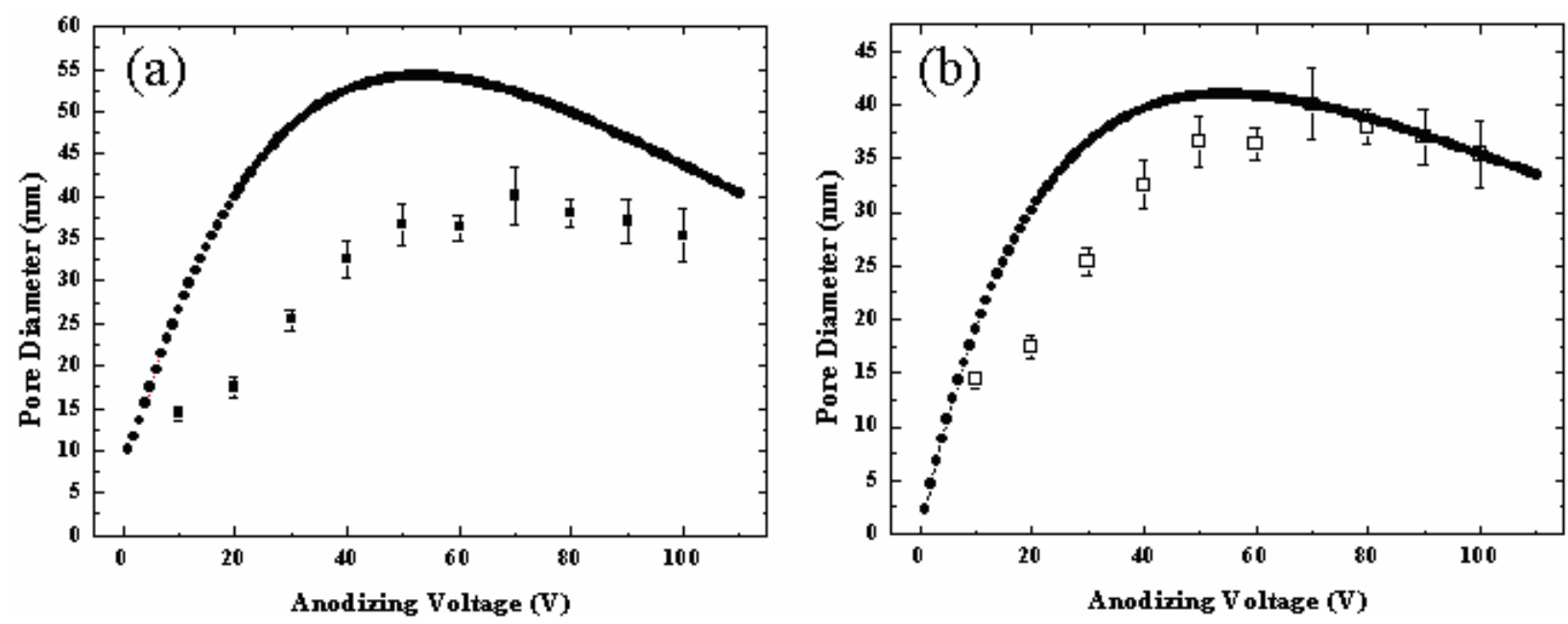

Figure 4-13. Plots of the estimated pore diameter versus the applied voltage for an ATO film anodised in ethylene glycol containing 0.3 wt $\% \mathrm{NH}_{4} \mathrm{~F}$ and $2 \mathrm{wt} \%$ water: (a) when barrier thickness has an exponential relation with applied voltage, (b) when current density has an exponential relation with applied voltage. The squares with standard deviation as the error bars corresponding to the experimental measurements.

Figure 4-13 shows the theoretical plots of the pore diameter versus the applied voltage for ATO. It is interesting to see that the curves have a peak position at about $55 \mathrm{~V}$ and $53 \mathrm{~V}$ in Figure 4-13a and $b$ respectively. Which implies that further increase of the applied voltage from the peak position will result in a reduction of the pore dimension, in contrast to the common accepted concept that pore diameter always has a positive correlation with applied voltage in the formation of anodic metal oxide films. Though the experimental data doesn't fit the theoretical perfectly, it clearly confirmed the existence of a maximum pore diameter at a voltage in the range from $50 \mathrm{~V}$ to $70 \mathrm{~V}$. Figure 4-14 shows the typical pore diameters variation for ATO 
produced in ethylene glycol containing $0.3 \mathrm{wt} \% \mathrm{NH}_{4} \mathrm{~F}$ and $2 \mathrm{wt} \%$ water with different anodising voltages of $20,40,70$, and $100 \mathrm{~V}$.
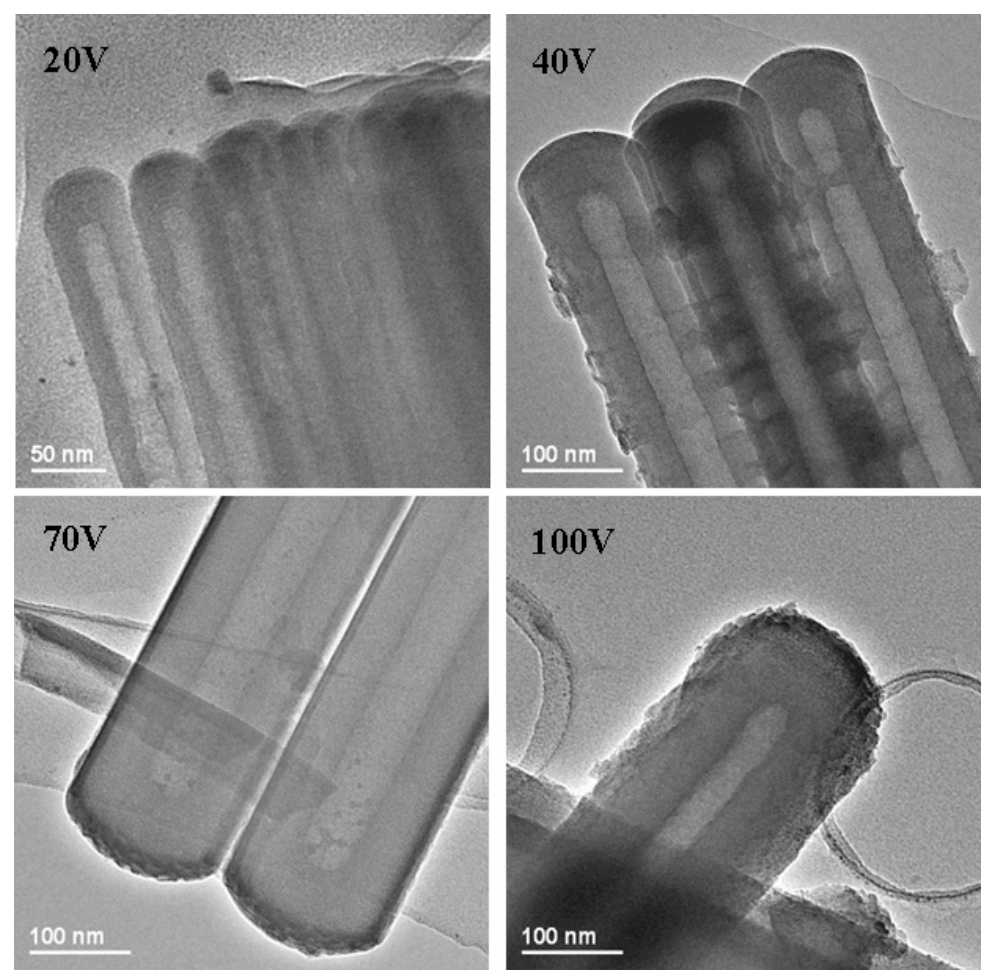

Figure 4-14. Typical pore diameter for ATO produced in ethylene glycol containing $0.3 \mathrm{wt} \%$ $\mathrm{NH}_{4} \mathrm{~F}$ and $2 \mathrm{wt} \%$ water at $20 \mathrm{~V}, 40 \mathrm{~V}, 70 \mathrm{~V}$, and $100 \mathrm{~V}$. The average diameters at these four voltages are $17.5 \mathrm{~nm}, 32.5 \mathrm{~nm}, 40.1 \mathrm{~nm}$ and $35.4 \mathrm{~nm}$ respectively.

The deviation between the experimental results might come from two aspects. Firstly, the error could come from the measurement of the pore diameters. In the present work, 20 nanotubes were measured for the ATO films produced at each anodising voltage. To get a more precise measurement for the pore diameter, it is better to collect as many nanotubes as possible. On the other hand, as mentioned in the previous work, ${ }^{249}$ neglecting of the $\mathrm{O}_{2}$ bubbles produced at the electrolyte/oxide interface during the anodisation would cause the measured current density to be larger than the real ionic current density.

In addition, local current heating could increase the dissolution rate of titanium oxide, which might induce further deviation. In fact, as the anodising voltage comes to a value above $120 \mathrm{~V}$, 
the local temperature rising could be rather serious that the pore diameter would be widen dramatically due to the much strengthened dissolution of the ATO films.

\subsection{Dissolved $\mathrm{Ti}^{4+}$ cations from two interfaces}

The proposed model also allows us to estimate the molar ratio of the titanium cations dissolved from $\mathrm{TiO}_{2}$ barrier layer $\left(\mathrm{Ti}_{\text {diss }}\right)$ to the total titanium cations $\left(\mathrm{Ti}_{\text {lost }}\right)$ lost into the bulk solution during the film growth. The latter is the sum of $T i_{d i s s}$ and the moles of titanium cations directly ejected from the hydroxide/metal interface into the electrolyte $\left(T_{i j e c t}\right)$. Using the hexagonal model,

$$
\frac{T i_{\text {diss }}}{T i_{\text {lost }}}=\frac{\pi \times r^{2} \times \Delta L \times D_{T i(O)}}{\frac{\sqrt{3}}{2} a^{2} \times \Delta L \times D_{T i(M)}-\left(\frac{\sqrt{3}}{2} a^{2}-\pi \times r^{2}\right) \times \Delta L \times D_{T i(O)}}
$$

where $D_{T i(M)}$ is the molar density of Ti in metal $\left(\sim 0.094 \mathrm{~mol} / \mathrm{cm}^{3}\right), D_{T i(O)}$ is the molar density of Ti in oxide $\left(\sim 0.053 \mathrm{~mol} / \mathrm{cm}^{3}\right), r$ is the pore radius and $a$ the cell diameter. Considering

$$
P=\frac{2}{n+2}=\frac{\pi}{2 \sqrt{3}}\left(\frac{D_{\text {Pore }}}{D_{\text {Cell }}}\right)^{2}=\frac{2 \pi}{\sqrt{3}}\left(\frac{r}{a}\right)^{2}
$$

then,

$$
\frac{T i_{\text {diss }}}{T i_{\text {lost }}}=\frac{D_{T i(O)}}{\left(\frac{n}{2}+1\right) D_{T i(M)}-\frac{n}{2} D_{T i(O)}}=\frac{106}{41 n+188}
$$

When anodisation takes place at $30 \mathrm{~V}$, the porosity is about $11.0 \%$ and the corresponding $n$ is about 16.2. Consequently, only $12.4 \%$ of the total titanium cations lost during the anodisation are from the dissolution of $\mathrm{TiO}_{2}$ in the oxide/electrolyte interface, while $87.6 \%$ of the titanium cations leave the hydroxide/metal interface, migrate across the barrier layer and are ejected into 
the electrolyte without forming oxide.

\subsection{Crystallisation of anodic titanium oxide}

As-synthesized ATO nanotubular arrays are non-crystalline in both oxide and hydroxide layers. This property limits the application of the materials, since both the conductivity and the mechanical strength of these materials are low. It has been established that, compared to the amorphous and the rutile form of $\mathrm{TiO}_{2}$, the anatase phase of titanium dioxide is a preferred electrode material in dye sensitized solar cells, ${ }^{250}$ and has a higher photocatalytic activity. ${ }^{251-252}$ On the other hand, the rutile phase provides titania with better hydrogen-sensing capabilities. Furthermore, as we discussed above, due to the dehydration of titanium hydroxide, the fabricated ATO nanotubes are separated from each other, leading to a relative loose linkage of the nanotubes. The only connections between the nanotubes are the so-called O-rings (Figure 4-3) and a connection at the nanotube base (Figure 4-5d). A weak mechanical vibration could peel off the nanotubes from an ATO film easily or even cause a collapse of the whole array structure. To overcome this problem, crystallisation of the as-synthesized ATO has been performed in the present work.
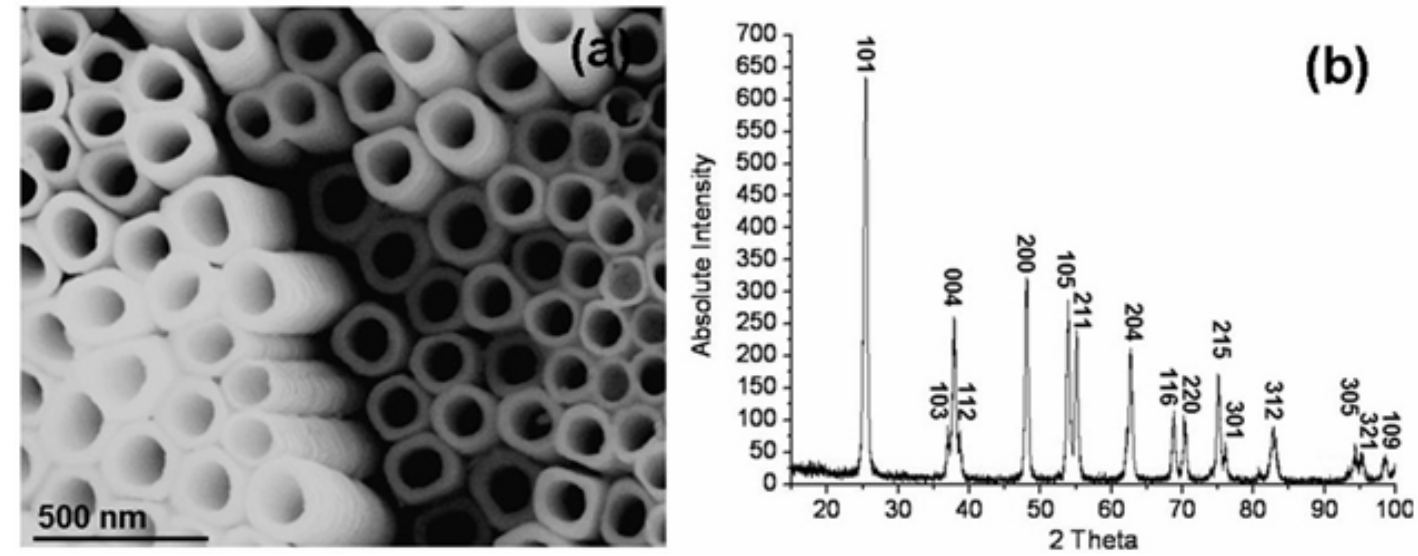

Figure 4-15. (a) SEM image of an ATO nanotubular array after annealing at $600{ }^{\circ} \mathrm{C}$. (b) Corresponding XRD pattern indexed onto the tetragonal anatase structure.

Based on the HRTEM studies, it was found the hydroxide layer can be partially crystallised 
into a polycrystalline state during dehydration enhanced by the electric field without any heat treatment. ${ }^{78}$ A large number of $d$-spacings measured from lattice fringes on HRTEM images indicated that these nanocrystallites are $\beta-\mathrm{TiO}_{2}$, monoclinic with $a=1.216, b=0.374, c=0.651$ and $\beta=107.29^{\circ}$. In the present work, it was found that the whole hydroxide layer including the small bridges connecting the nanotubes can be crystallised after annealing at $285^{\circ} \mathrm{C}$ for $24 \mathrm{~h}$ into a single crystal shell on the nanotubes, when some nanocrystallites were developed in the inner oxide layer. Annealing in air at $600{ }^{\circ} \mathrm{C}$ for $5 \mathrm{~h}$, the sizes of monocrystalline domains increased remarkably. It is interesting to see that the crystal phase after high temperature treatment is pure anatase, as all the XRD peaks can be indexed onto this tetragonal phase with the unit cell parameters of $a=0.378$ and $c=0.951 \mathrm{~nm}$. Importantly, the original morphology of nanotubular array is almost intact (Figure 4-15).
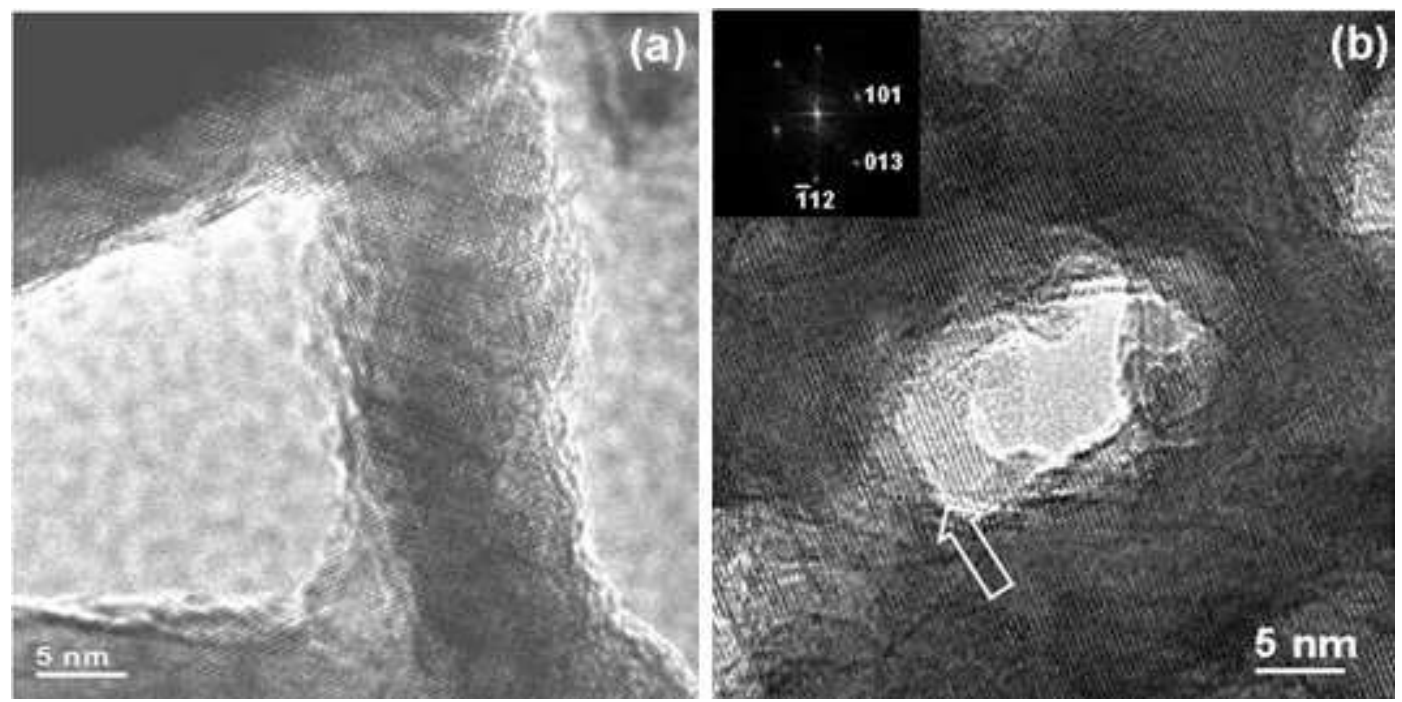

Figure 4-16. HRTEM images of small bridges connecting nanotubes after recrystallisation by annealing, showing (a) an intermediate state with oriented domains at $300{ }^{\circ} \mathrm{C} 2 \mathrm{~h}$, and (b) single crystal shell viewed down the [13-1] zone axis of anatase structure at $285{ }^{\circ} \mathrm{C} 24 \mathrm{~h}$. The arrow points two bridges overlapped along the view direction. The inset is the corresponding FFT diffraction pattern.

Figure 4-16a is a HRTEM image of a sample after annealing at $300{ }^{\circ} \mathrm{C}$ for $2 \mathrm{~h}$, showing a small bridge connecting two nanotubes with its structure approaching to a single crystal but many oriented domains can still be identified. This is an intermediate state of the recrystallisation 
process from polycrystalline to monocrystalline phases. Figure 4-16b is a typical HRTEM image of recrystallised shells of nanotubes after annealing at $285{ }^{\circ} \mathrm{C}$ for $24 \mathrm{~h}$. The image contrast pattern and the corresponding diffraction pattern, projected along the [13-1] zone axis of anatase, show that the whole area including bridges overlapped along the viewing direction as indicated by an arrow is monocrystalline. The HRTEM images from the same sample also suggested that polycrystallites were developed in the original oxide layer, leading to a smart material with polycrystalline titanium oxide nanotubes coated by a single crystal layer on the outer surface and connected by some small bridges with the same anatase phase. It is expected that further annealing may allow recrystallisation expanding from the outer surface to the inner surface via an Ostwald ripening process and eventually form a connected single crystal nanotubular array. This crystallisation process is similar to the recently established NARS route of crystal growth, ${ }^{253}$ i.e., crystal growth can follow a reversed route: nanoparticles, aggregation, surface recrystallisation and single crystals.

In summary, the newly established equifield model can be used to interpret the formation of anodic $\mathrm{TiO}_{2}$ nanotubular arrays with a hemispherical tube bottom and a self-ordering potential. It has also been revealed that the electric field enhanced dissociation of water followed by anion migration plays an important role in the formation of ATO films, i.e. governing the porosity and geometry of nanotubular arrays. The establishments of the relations between porosity and anodisation conditions enable the production of ATO films to be highly controllable and predictable. Crystallisation of the as-synthesized amorphous ATO into the anatase phase has been successfully achieved. On the other hand, as observed in experiments, $\mathrm{O}_{2}$ bubbles were often produced at the electrolyte/oxide interface during the anodisation, the ionic current density thus should be slightly smaller than the measured current density. In addition, local current heating could increase the dissolution rate of titanium oxide, which might induce further deviation. Further investigations about these effects and the physico-chemical properties of the crystalline ATO films are currently carried out in this laboratory. 


\section{Field Enhanced Dissociation of Water}

In the previous paragraphs, an equifield strength model has been established for elucidating the self-organisation of AAO growth. It has also been proposed that dissociation of water at the electrolyte/oxide interface is the key factor for governing the porosity $(P)$ of AAO films, in a very simple relation,

$P=3 /(n+3)$

where $n$ is introduced to indicate the amount of water that dissociates per mole of $\mathrm{Al}_{2} \mathrm{O}_{3}$ that is dissolved at the same time, according to the following reaction:

$\mathrm{Al}_{2} \mathrm{O}_{3}(s)+n \mathrm{H}_{2} \mathrm{O}(l) \rightarrow 2 \mathrm{Al}^{3+}(a q)+(3+n-x) \mathrm{O}^{2-}(s)+x \mathrm{OH}^{-}(s)+(2 n-\mathrm{x}) \mathrm{H}^{+}(a q)$

Such water dissociation become even more important during anodisation of titanium, since it has been discovered recently that the product of water dissociation, $\mathrm{OH}^{-}$, forms a hydroxide layer in between the oxide layer and the metal substrate. ${ }^{249}$ Generally speaking, field-enhanced water dissociation must play an important role in anodic oxide formation, but has not been extensively investigated. From the observed porosities as function of the anodisation conditions (in particular the current density), an important prediction of this model is that the rate of this dissociation process at such a liquid/solid interface should increase with the strength of the applied electric field.

Thus it is worth investigating the rate of water dissociation at the electrolyte/oxide interface in the presence of a high electric field by a combined methodology of computational and experimental chemistry. For this purpose, we used quantum chemistry to model the $\mathrm{OH}$ bond strength of water as a function of the applied external electric field. Inclusion of such an electric field in the computations is straightforward, and a number of ab initio and density functional theory (DFT) studies have addressed the field-dependence of structures,${ }^{254}$ dipole moments, ${ }^{255}$ bond strengths, ${ }^{256,257}$ or reactivities ${ }^{258-261}$ of molecules or solids. Field effects on the H-bond network of liquid water ${ }^{262}$ and on mobility of hydronium ions therein ${ }^{263}$ have been modelled 
theoretically, but to our knowledge, the question of the bond strength of water in an external field has not been addressed yet.

It should be pointed out here that, due to the limitation of knowledge in the computational chemistry, I did not make a great contribution in the theoretical computation work. This part of calculation was mainly done by Prof. M. Bühl from the School of Chemistry in University of St Andrews, although we jointly established the project, had several discussions and supplied experimental data for comparison.

\subsection{Theoretical computation for the field present water dissociation}

First-principles predictions of thermodynamic and kinetic parameters of heterolytic bond dissociations in polar condensed media are a daunting challenge. The simplest possible model system for the effect under scrutiny is the water dimer dissociating into an $\mathrm{OH}^{-} \cdot \mathrm{H}_{3} \mathrm{O}^{+}$ion pair, where the hydroxide would remain bound at the oxide surface, later migrating across the oxide layer to the oxide/metal interface, and the hydronium ion would eventually be released into the electrolyte solution. For this and somewhat more elaborate models (see Figure 5-1), we evaluated heterolytic dissociation profiles quantum-mechanically at suitable levels (e.g. B3LYP/6-311+G(d,p) in a Polarizable continuum) in the presence of an external field suitably aligned with the breaking $\mathrm{OH}$ bond.

\subsubsection{Details of the computation}

The systems shown in Figure 5-1 were first fully optimized without electric field in the gas phase at the B3LYP/6-311+G** level, ${ }^{264-268}$ where all are true minima. Subsequently, dissociation profiles were computed at the same level by elongating the $\mathrm{OH}$ bond successively in steps of $0.1 \AA$ and optimizing all other geometrical parameters of the water dimer moiety, except for the $\mathrm{O}-\mathrm{H} \cdots \mathrm{O}$ bond angle, which was kept fixed at the value of the respective minimum (to prevent the forming $\mathrm{H}_{3} \mathrm{O}^{+}$from "bending back" to the hydroxide by closing this angle). For the cluster models, the $\mathrm{Al}_{4} \mathrm{O}_{6}$ moieties were also kept fixed in their fully optimized structures. Next, 
single-point energy computations were performed on these partially optimized gas-phase structures employing the Polarizable continuum model (PCM) by Tomasi and coworkers, ${ }^{269-271}$ using a dielectric constant of $\varepsilon=6$ (see below) and Merz-Kollman atomic radii (together with explicit hydrogens) for the construction of the cavity. Finally, these single-point energy computations were repeated in the presence of an electric field of a given strength $F$ and fixed orientation with respect to the breaking $\mathrm{OH}$ bond. The stabilization energies $\Delta E$ were evaluated as differences between the latter energies (at an $\mathrm{O} \cdots \mathrm{H}$ distance of $2.0 \AA$ ) and those in the field-free cases, where a negative sign denotes a stabilization in the field. For the free water dimer, the same protocol was followed also at the MP2/6-311+G** level. All computations were performed using the Gaussian03 program package. ${ }^{272}$
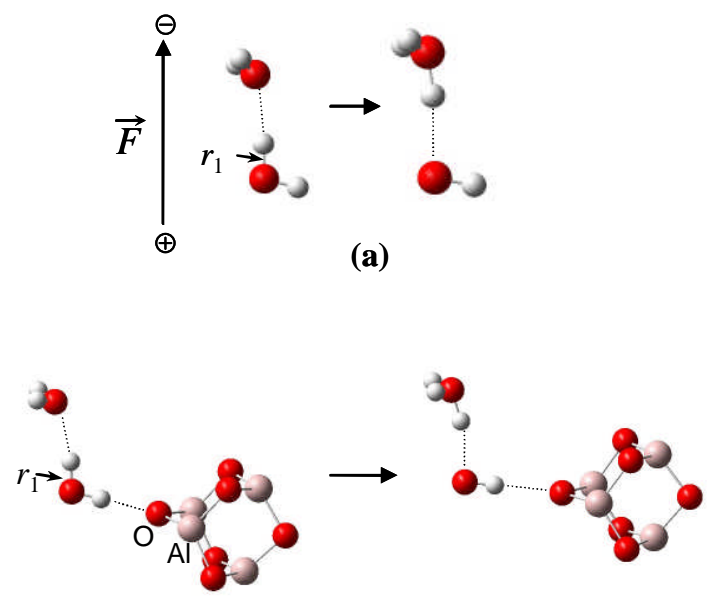

(b)

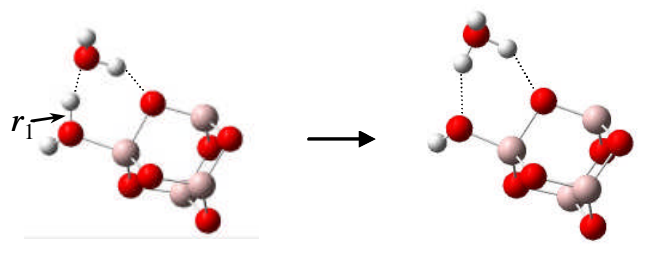

(c)

Figure 5-1. Models to study the effect of an electric field on the dissociation of an $\mathrm{OH}$ bond (labeled $r_{1}$ ) in a water molecule: (a) water dimer, (b) water dimer donating a $\mathrm{H}$ bond to an $\mathrm{Al}_{4} \mathrm{O}_{6}$ cluster, (c) water dimer coordinated via the $\mathrm{O}$ atom to an $\mathrm{Al}_{4} \mathrm{O}_{6}$ cluster. 


\subsubsection{Results of the computation}

To illustrate the dependence of the $\Delta E$ values on the strength of the external field, the dissociation profiles for perfect alignment of field and $\mathrm{OH}$ bond are plotted for the water dimer in Figure 5-2. Due to the imperfections of this simplistic model for water dissociation, the resulting profiles themselves are not realistic in a quantitative sense. What is interesting and significant, however, is the extent of the lowering of the energies with increasing field strength. In actual liquid water, an $\mathrm{OH}$ distance of ca. $1.8 \AA$ can be considered as effectively broken. This is evident, for instance, from constrained Car-Parrinello molecular dynamics simulations of the bulk liquid, where the free energy of O-H dissociation starts to level off at such a distance, ${ }^{273,274}$ and the well-known hopping mechanism for proton transport sets in. To be on the safe side, we assumed a distance of $2.0 \AA$ for full dissociation and evaluated the stabilization energy with respect to the field-free case, $\Delta E$, at this value for $r_{1} \cdot{ }^{275}$ Figure 5-3a shows the almost perfect linearity between the resulting $\Delta E$ values and the field strength $F$ in this orientation. If the field is aligned such that the dissociating $\mathrm{O}$ and $\mathrm{H}$ atoms are at the positive and negative poles, respectively, $\Delta E$ is negative.

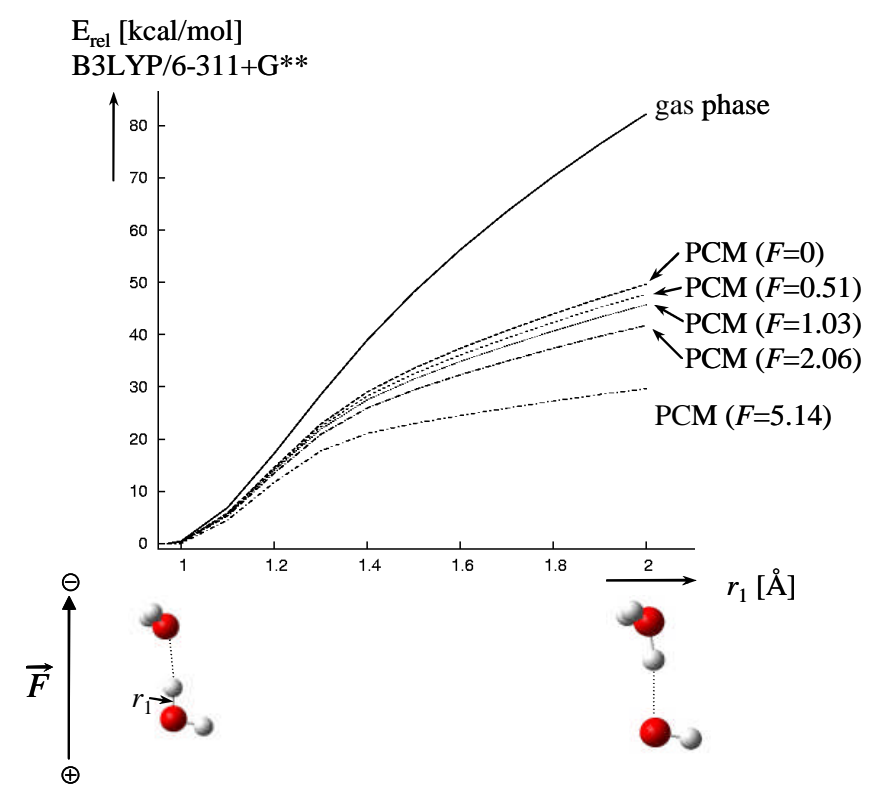

Figure 5-2. Dissociation profiles for the $\mathrm{OH}$ bond in the water dimer $(\alpha=0)$ in the gas phase and in a Polarizable continuum $\left(\varepsilon=6, F\right.$ in $\left.\mathrm{V} \mathrm{nm}^{-1}\right)$ 
The orientation dependence of $\Delta E$ as function of $\alpha$ is shown for the water dimer in Figure 5-3b, employing an intermediate value of $F\left(1.03 \mathrm{~V} \mathrm{~nm}^{-1}\right)$. The computed data (black dots) can be approximated reasonably well by a simple cosine function (solid line). ${ }^{276}$ Assuming the same holds for all field strengths this affords the expression:

$\Delta E \approx-\mathrm{c}_{1} F \cos \alpha$

where $\mathrm{c}_{1}$ is a positive constant. A linear fit of $\Delta E$ values obtained at $F=0.51,1.03,2.06$, and $5.14 \mathrm{~V} \mathrm{~nm}^{-1}$ yields $\mathrm{c}_{1}=3.90 \mathrm{kcal} \mathrm{nm} \mathrm{mol}{ }^{-1} \mathrm{~V}^{-1} \cdot{ }^{277,278}$ The choice of $\varepsilon$ in these computations is not overly critical. For instance, for the water dimer at $\alpha=0$, using $\varepsilon=78.4$ instead of 6 yields $\mathrm{c}_{1}=$ $4.03 \mathrm{kcal} \mathrm{nm} \mathrm{mol}^{-1} \mathrm{~V}^{-1}$ instead of $3.90 \mathrm{kcal} \mathrm{nm} \mathrm{mol}^{-1} \mathrm{~V}^{-1}$. Essentially the same value, 3.97 $\mathrm{kcal} \mathrm{nm} \mathrm{mol}{ }^{-1} \mathrm{~V}^{-1}$, is obtained at the MP2/6-311+G(d,p) level.
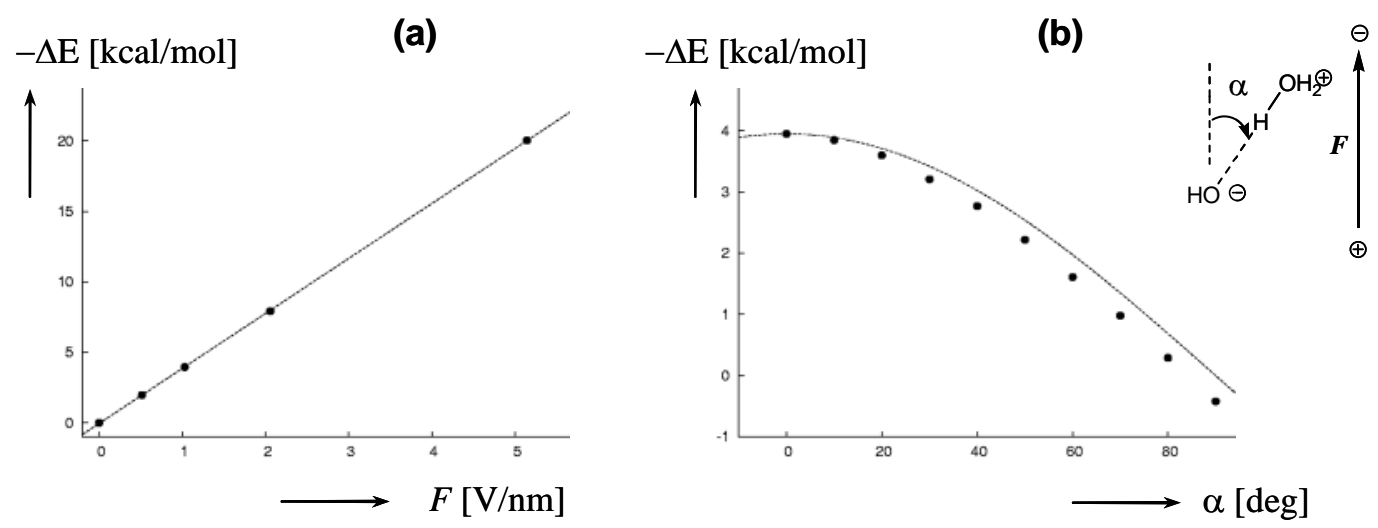

Figure 5-3. Stabilisation energy $\Delta E$ of the water dimer (at $r_{1}=2.0 \AA$ and $\varepsilon=6$ ) as a function of (a) the field strength $F$ (for $\alpha=0$ ) and (b) the angle $\alpha$ (at $F=1.03 \mathrm{~V} \mathrm{~nm}^{-1}$ ).

The heterogeneous systems in the actual AAO process pose the additional complication that the microstructure of the porous aluminium oxide at the interface with the solution is rather ill-defined. DFT computations for an ideal periodic (0001) surface of $\alpha-\mathrm{Al}_{2} \mathrm{O}_{3}$ have identified two favorite bonding modes of water at high coverage, namely donating an $\mathrm{OH}$ bond to a surface $\mathrm{O}$ atom or coordinating via $\mathrm{O}$ to an $\mathrm{Al}$ atom of the surface. ${ }^{279}$ We have modeled this situation by attaching the dissociating water dimer in a corresponding manner to an $\mathrm{Al}_{4} \mathrm{O}_{6}$ cluster (which had been used before as simple alumina $\operatorname{model}^{280}$ ), see Figure $5-1 b$ and $c$. The $c_{1}$ values obtained in 
these cases are 4.01 and $3.29 \mathrm{kcal} \mathrm{nm} \mathrm{mol}^{-1} \mathrm{~V}^{-1}$, respectively, i.e., qualitatively very similar to that obtained for the free water dimer.

In order to model effects of explicit hydration on the dissociation profile, we also studied the cyclic water tetramer in an analogous fashion. Starting from the $S_{4}$ symmetric minimum and elongating $r_{1}$ successively (see Figure $\left.5-4\right)$, it was necessary to fix the opposite $\mathrm{O} \cdots \mathrm{H}$ distance $\left(r_{3}\right.$ in Figure 5-4), in order to prevent a cyclic concerted transfer of the four bridging $\mathrm{H}$ atoms, which would eventually restore the neutral water tetramer rather than maintain the desired $\mathrm{H}_{3} \mathrm{O}_{2}{ }^{-} \mathrm{H}_{5} \mathrm{O}_{2}{ }^{+}$ ion pair. No angle constraint was imposed in this case.

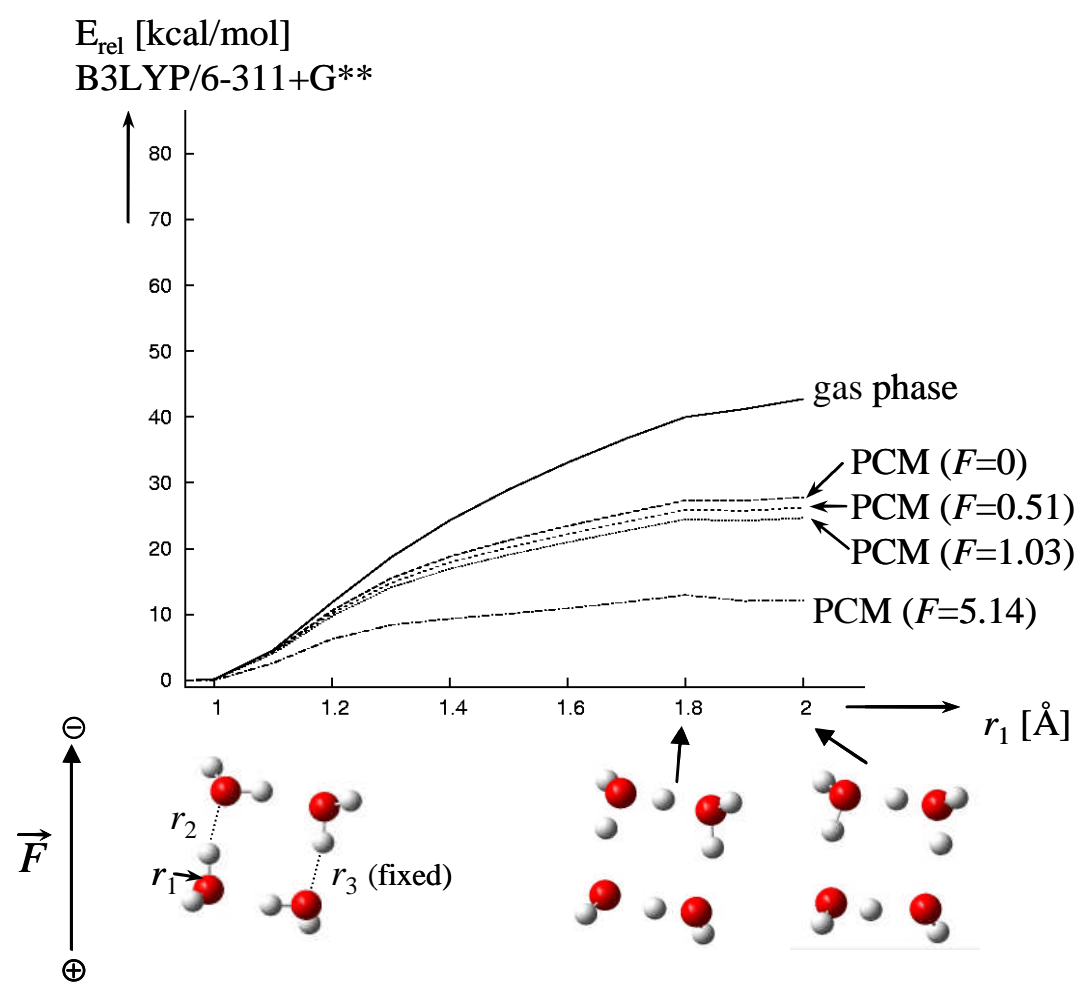

Figure 5-4: Dissociation profiles for an $\mathrm{OH}$ bond in the water tetramer in the gas phase and in a Polarizable continuum $\left(\varepsilon=6, F\right.$ in $\left.\mathrm{V} \mathrm{nm}^{-1}\right)$

For this tetramer model, $\mathrm{H}$ transfer effectively occurs at $r_{1}=1.8 \AA$, at which point the two unsymmetrical $\mathrm{OH} \cdots \mathrm{O}$ bonds at the "upper" and "lower" sides (referring to the orientation depicted at the bottom of Figure 5-4) invert, i.e., where they switch to the respective O $\cdots \mathrm{HO}$ orientations. Using the same fitting procedure as for the water dimer, $\mathrm{c}_{1}=2.96 \mathrm{kcal} \mathrm{nm} \mathrm{mol}{ }^{-1} \mathrm{~V}^{-1}$ 
is obtained for the tetramer. Note that beyond $r_{1}=1.8 \AA$, the potential energy curves in Figure 5-4 visibly level off, which is in good qualitative accord with sophisticated CPMD simulations. ${ }^{273,274}$ In the field-free case, and using the dielectric constant of water, a dissociation energy around ca. $27 \mathrm{kcal} / \mathrm{mol}$ is obtained for our tetramer model, which can be compared with free energies of water dissociation in the bulk from CPMD/BLYP simulations (ca. $17 \mathrm{kcal} / \mathrm{mol}$ ) and experiment $(21.4 \mathrm{kcal} / \mathrm{mol}$, see [refs. 273,274] and references cited therein). Given the simplicity of our model computations and the neglect of temperature and entropy effects therein, the agreement with other theoretical and with experimental data is actually quite reasonable. It is, however, not the dissociation energy as such that we attempt to model, but rather the effect of the electric field on it.

When the water-dissociation energy is lowered by an amount according to eq. (5.3), and when all thermal and entropic contributions arising from the external field are neglected, the relative rate enhancement for the dissociation process is:

$\kappa / \kappa_{0}=\exp \left(\mathrm{c}_{1} F \cos \alpha / \mathrm{RT}\right)$

where $\kappa_{\text {. }}$ and $\kappa_{0}$ are the absolute rate constants with and without field, respectively. The ratios $\kappa / \kappa_{0}$ according to eq. (5.4) are plotted in Figure 5-5 using the highest of the $\mathrm{c}_{1}$ values as an upper limit, and a few representative values for $\alpha$, estimated from idealized orientations of water molecules adsorbed on a surface (Scheme 5-1). These orientations refer to an extended planar surface perpendicular to the field (where $\mathrm{X}$ can be either $\mathrm{O}$ or $\mathrm{Al}$ ), rather than to the simplistic models in Figure 5-1b,c. Evidently, the rate-enhancing effect of the field can be substantial, but should also be very sensitive to the orientation of the water molecules.

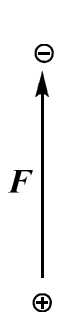

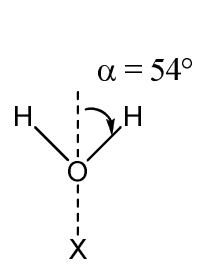

(b)

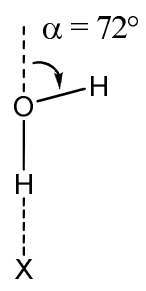

(c)

Scheme 5-1. Idealized orientations of water molecules on a surface. 


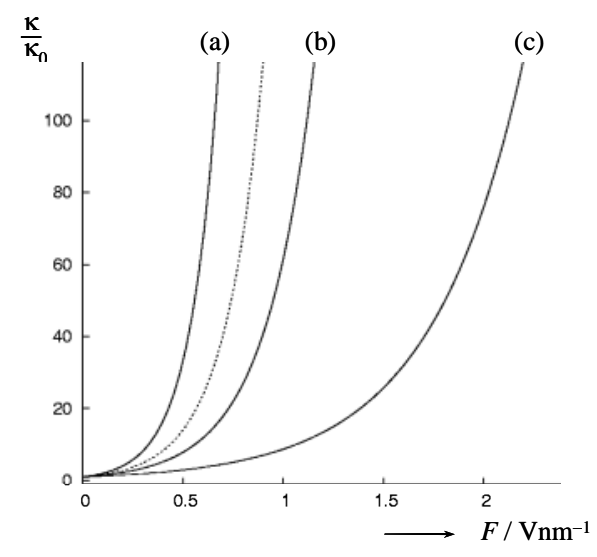

Figure 5-5. Plot of $\kappa / \kappa_{0}$ according to eq. (5.4) $(\mathrm{T}=288 \mathrm{~K})$, using $\mathrm{c}_{1}=4.01 \mathrm{kcal} \mathrm{nm} \mathrm{mol}^{-1} \mathrm{~V}^{-1}$, and (a) $\alpha=0$; (b) $\alpha=54^{\circ}$, and (c) $\alpha=72^{\circ}$ (the latter two correspond to the orientations in Scheme 1). Dotted line: experimental estimate for $\mathrm{c}_{1} \cos \alpha=3.0 \mathrm{kcal} \mathrm{nm} \mathrm{mol}^{-1} \mathrm{~V}^{-1}$ (see text).

\subsection{The experimental fit for the theoretical computation}

In order to relate these microscopic results to the macroscopic observables, we now consider our working hypothesis for the anodisation process in more detail. The total oxygen anionic current $j$ across the barrier oxide at the pore base consists of two parts, $j_{\text {oxide }}$ from the electric-field enhanced dissolution of the oxide at the oxide/electrolyte interface, and $j_{\text {water }}$ from the dissociation of water:

$j=j_{\text {water }}+j_{\text {oxide }}$

where $j_{\text {water }} / j_{\text {oxide }}=n / 3$ ( $n$ is defined by eq. (5.2)), since the current density is proportional to the anions created from the surface reactions. The porosity $P$ of the anodic oxide film should be related to these current densities according to

$P=\frac{n}{n+3}=\frac{j_{\text {oxide }}}{j_{\text {oxide }}+j_{\text {water }}}=\frac{j_{\text {oxide }}}{j}$

and 


$$
\frac{j_{\text {water }}}{j}=\frac{n}{n+3}=1-\frac{n}{n+3}=1-P
$$

The oxygen-anion current attributed to the dissociation of water, $j_{\text {water }}$, should have the same relationship with the local electric field strength $F$ at the oxide/electrolyte interface as the dissociation rate of water (eq. (5.4)). Neglecting the temperature change due to the local heating at the barrier oxide layer, there is

$j_{\text {water }} / j^{0}{ }_{\text {water }}=\exp \left(\mathrm{c}_{1} F \cos \alpha / \mathrm{RT}\right)$

where $j^{0}{ }_{\text {water }}$ denotes the oxygen-anion current attributed to the dissociation of water in the field-free case.

Table 5-1. Experimental data of anodisation $(\mathrm{T}=288 \mathrm{~K})$ : applied voltage $(U)$, measured current $(j)$, field strength $(E)$ and measured porosity $(P)$.

\begin{tabular}{lllllll}
\hline No. & $U(\mathrm{~V})$ & $j(\mathrm{~mA})$ & $p(\%)$ & $j_{\text {water }}(\mathrm{mA})^{\mathrm{a}}$ & $F\left(\mathrm{Vnm}^{-1}\right)^{\mathrm{a}}$ & Ref. \\
\hline 1 & 5 & 0.39 & 35.0 & 0.254 & 0.588 & 93 \\
2 & 10 & 0.80 & 20.1 & 0.638 & 0.656 & 93 \\
3 & 20 & 1.85 & 13.6 & 1.600 & 0.736 & 93 \\
4 & 30 & 3.29 & 10.8 & 2.934 & 0.790 & 93 \\
5 & 40 & 5.45 & 9.4 & 4.938 & 0.838 & 93 \\
6 & 20 & 2.0 & $17.5^{\mathrm{a}}$ & 1.65 & 0.743 & 225 \\
7 & 30 & 3.0 & $13.6^{\mathrm{a}}$ & 2.59 & 0.782 & 225 \\
8 & 40 & 5.5 & $9.4^{\mathrm{a}}$ & 4.98 & 0.839 & 225 \\
9 & 40 & 5 & 10 & 4.50 & 0.83 & 70,235 \\
10 & 110 & 30 & 3.3 & 29.0 & 1.0 & 70 \\
\hline
\end{tabular}

${ }^{a}$ Derived value, see text. 
The main difficulty is now to relate the externally applied voltage $U$ to the local field strength $F$ at the oxide/water interface. As the aluminium-oxide layer is sandwiched by two effective conductors, aluminium metal and electrolyte solution, it is reasonable to assume that essentially all the voltage $U$ is acting across the oxide layer of thickness $d_{C}$. With this simple model, and using experimental data for $j$ and $P$ (which are given in Table 5-1) together with previously established empirical relationships, values for $F$ can be deduced. Using these raw data (which are included in Table 5-1) to fit eq. (5.8) affords $6.3 \mathrm{kcal} \mathrm{nm} \mathrm{mol}^{-1} \mathrm{~V}^{-1}$ as first estimate for the factor $\mathrm{c}_{1} \cos \alpha$.

The data were derived as follows: the fraction $j_{\text {water }}$ in the total current strength, $j$, is given by

$j_{\text {water }}=j(1-P)$,

as obtained from eq. (5.7). An empirical relation between $j$ and $F$ has been derived previously, namely eq. (17) in [ref. 225]:

$j=7.94 \cdot 10^{-4} \exp (10.45 F)$

where $j$ and $F$ are given in units of $\mathrm{mA}$ and $\mathrm{V} \mathrm{nm}^{-1}$, respectively. When no porosities have been reported, these have been estimated via the relationship derived in eq. (19) of [ref. 225]:

$P=21.3 \exp (-6.46 F)$

Figure 5-6 shows a plot of the resulting experimental $j_{\text {water }}$ values vs. the derived data for $F$ collected in Table 5-1, together with a fit according to

$j_{\text {water }}=j^{0}$ water $\exp \left(\mathrm{c}_{1} F \cos \alpha / \mathrm{RT}\right)$

as rearranged from eq. (5.8). The fitted values for $j^{0}$ water and $\mathrm{c}_{1} \cos \alpha / \mathrm{RT}$ are $4.8(3) \cdot 10^{-4} \mathrm{~mA}$ and 11.02(4), respectively (in parentheses: standard deviations). From the latter value, $\mathrm{c}_{1} \cos \alpha=6.3$ kcal nm mol ${ }^{-1} \mathrm{~V}^{-1}$ is obtained at $\mathrm{T}=288 \mathrm{~K}$. 


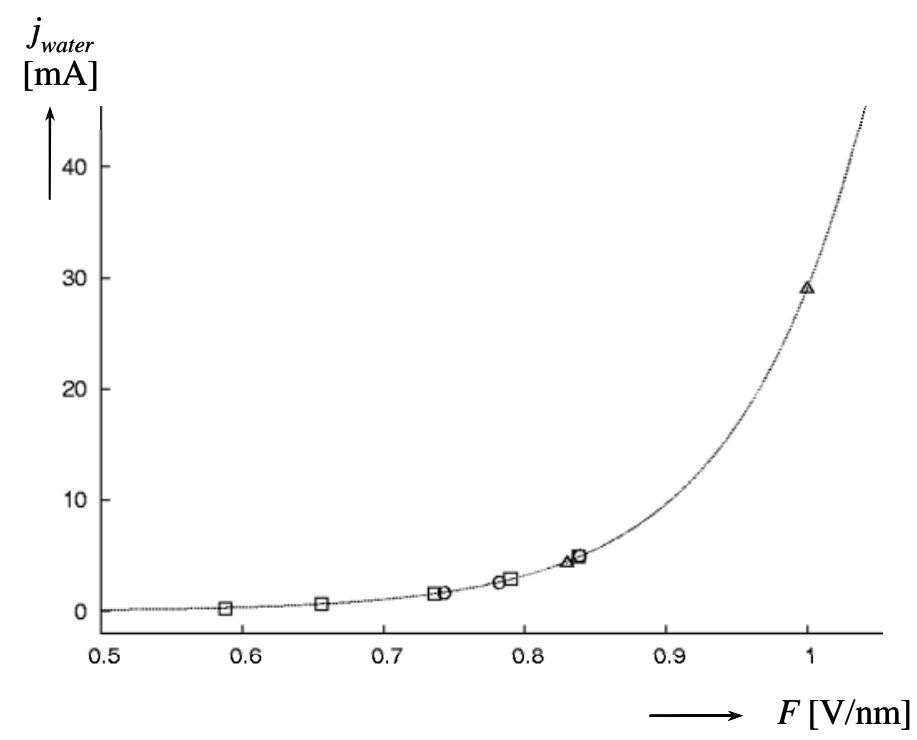

Figure 5-6. Plot of experimentally derived oxygen anion currents due to water dissociation, $j_{\text {water }}$, vs. electric field strengths $F$, together with an exponential fit. Squares, circles, and triangles are based on data taken from references 93,225 , and $(70,235)$ respectively.

To describe the local conditions at the phase boundary, it must be taken into account that a double layer with a characteristic Debye length and dielectric constant $\varepsilon_{d l}$ is formed at the oxide-electrolyte interface. From the continuity requirement of the normal component of the electric displacement at the oxide/electrolyte interface, and neglecting the effect of specific ionic adsorption, ${ }^{89}$ the following correction for $F$, the field strength in the double layer, is obtained:

$F \approx \frac{U}{d_{C}} \frac{\varepsilon_{\mathrm{Al}_{2} \mathrm{O}_{3}}}{\varepsilon_{d l}}$

It is well-established in electrochemistry that at an interface with a solid, the dielectric permittivity $(\varepsilon)$ of water is not the same as that in the bulk liquid. Rather, $\varepsilon$ has its smallest value $\varepsilon_{\min }$ (ca. 4-6) at the first layer at the water/solid interface and increases with the distance from the surface, up to $\varepsilon_{\max }=78$ in the bulk: ${ }^{84,281-283}$ 
$\varepsilon(z)=\varepsilon_{\max }\left[1+\left(\varepsilon_{\max } / \varepsilon_{\min }-1\right) \exp \left(-z / L^{-1}\right)\right]^{-1}$

where $z$ is the distance from the surface and $\mathrm{L}^{-1}$ is the Debye length related to the double layer in a given electrolyte. According to the Debye-Hückel theory, the Debye length is given as:

$L^{-1}=\sqrt{\frac{\varepsilon_{0} \varepsilon_{r} k_{B} T}{e^{2} \sum n_{i}^{\infty} Z_{i}^{2}}}$

where $\varepsilon_{0}$ and $\varepsilon_{\mathrm{r}}$ are the vacuum permittivity and the relative permittivity of the bulk electrolyte, respectively, $k_{B}$ is the Boltzmann constant, $T$ the absolute temperature, $e$ the electronic unit charge, $n$ the ion density in the bulk solution, and $Z i$ represents the ion valency. The sum runs over all species of ions present. In the present work for the electrolyte of $0.3 \mathrm{M}$ oxalic acid with a $\mathrm{pH}=1.2$, the calculated thickness of the Debye length is about $1.2 \mathrm{~nm}$. In agreement with previous work, ${ }^{84,282}$ we find that such a Debye length of ca. $1 \mathrm{~nm}$ corresponds to a dielectric constant of about 6 of the electrolyte at the oxide and electrolyte interface (this $\varepsilon$ value was also employed for the Polarizable continuum in the quantum-chemical computations discussed above). A plot of the dielectric permittivity of the electrolyte as a function of the distance from the surface is shown in Figure 5-7. Together with the permittivity of the bulk oxide taken as $\varepsilon_{\mathrm{Al}_{2} \mathrm{O}_{3}} \approx 9$, a refined empirical estimate for $\mathrm{c}_{1} \cos \alpha=4.2 \mathrm{kcal} \mathrm{nm} \mathrm{mol}^{-1} \mathrm{~V}^{-1}$ is obtained.

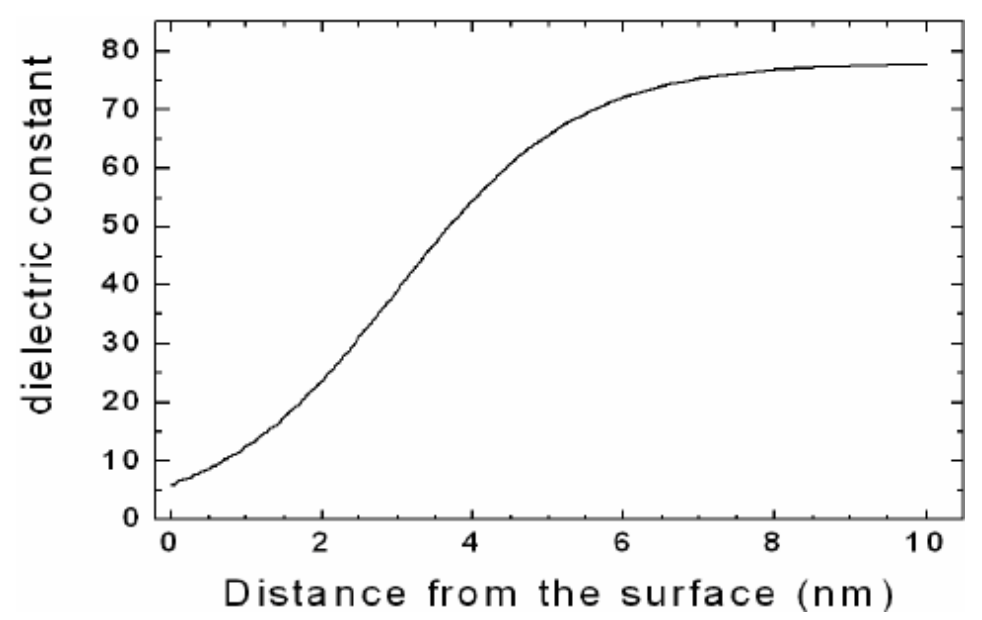

Figure 5-7. Distance dependence of the dielectric permittivity at the oxide/water interface according to eqs. (5.14) and (5.15). 
A further refinement of the local field strengths at the sites of the adsorbed water molecules is possible by considering that in a pure dielectric, this local field will be increased due to the response of the dielectric to the external field (i.e. due to the polarization of the medium). For example, in a spherical cavity within a uniform dielectric, the local field $F_{\text {loc }}$ is related to the external field $F_{\text {ext }}$ by ${ }^{84}$

$F_{\mathrm{loc}}=F_{\mathrm{ext}} \cdot 3 \varepsilon /(2 \varepsilon+1)$

Thus, $F_{\text {loc }}$ is enhanced by a factor approaching 1.5 in very polar environments. For $\varepsilon=6$, the empirical estimate for $\mathrm{c}_{1} \cos \alpha$ would be multiplied by $(2 \varepsilon+1) / 3 \varepsilon=0.722$, resulting in a value of $3.03 \mathrm{kcal} \mathrm{nm} \mathrm{mol} \mathrm{V}^{-1}$ for this quantity. This value is now in between the upper limit and the value corresponding to $\alpha=54^{\circ}$ modelled computationally (see dotted line in Figure 5-5). ${ }^{284}$ In view of the many assumptions and approximations that are involved, this apparent semi-quantitative accordance between theory and experiment should probably not be over interpreted. Nevertheless, both are in excellent qualitative agreement as to the order of magnitude of the underlying effect. Our results thus can be taken as strong evidence for the proposed models of equifield strength and field-enhanced dissociation of water at the growing oxide surface.

In summary, with the help of Prof. M. Bühl in the quantum-chemical model computations, it is showed that an external electric field can facilitate the heterolytic dissociation of properly oriented water molecules significantly. This fundamental finding strongly supports a previously proposed new mechanistic model for the electrochemical formation of porous aluminium oxide, which accounts for the decreasing porosity of the product with increasing applied voltage. Assuming that suitable orientations of water molecules are brought about by adsorption at the oxide surface, and employing physically reasonable relations between macroscopic and local electric field strengths, a remarkably good accord is found between key quantities derived from the quantum-chemical models and the experimental data. Arguably, this conceptual link between the fundamental response of a molecule to an external stimulus and the property of a material that is of interest for technological applications holds much promise for further exploration. 


\section{Ionic Nanoconvection in the Early Stage of Anodisation}

In the previous paragraphs an equifield strength model was proposed to explain the formation of the ordered pores in AAO and ATO when the pores grow in depth. ${ }^{78}$ However, some degree of pore ordering could appear at very early stage of anodisation, when possible pore-pore interaction is very weak. ${ }^{26}$ Figure 6-1 shows a SEM image of AAO with 2 min anodisation in 0.3 $\mathrm{M} \mathrm{H}_{2} \mathrm{C}_{2} \mathrm{O}_{4}$ electrolyte using anodisation voltage of DC $40 \mathrm{~V}$. With such a short time, the depth of the pores is very short, but partial ordering of the pores can be clearly seen. This observation guided us to look for another driving force for the pore ordering at the very early stage of anodisation of valve metals.

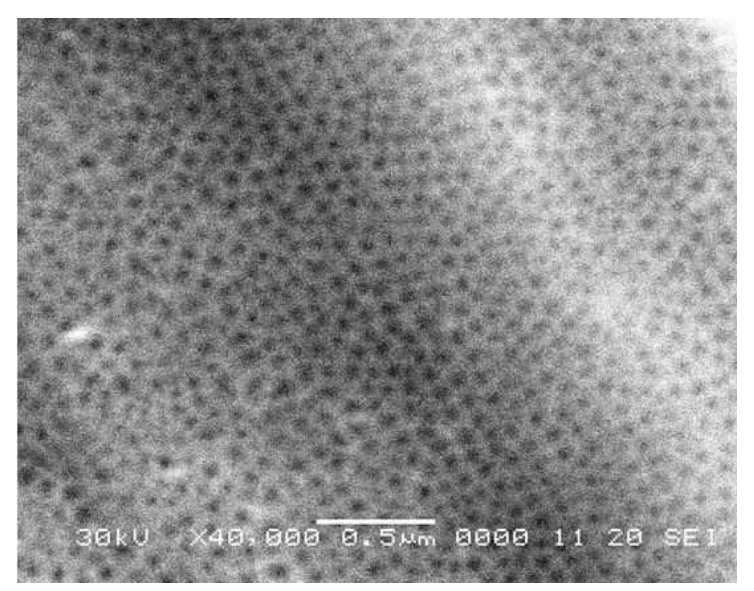

Figure 6-1. SEM image of AAO with 2 min anodisation in $0.3 \mathrm{M} \mathrm{H}_{2} \mathrm{C}_{2} \mathrm{O}_{4}$ electrolyte using anodisation voltage of DC $40 \mathrm{~V}$.

The formation of spatial patterns by convection of a fluid with hydrodynamic instabilities is commonly observed in various circumstances. The Rayleigh-Benard convection model established nearly one century ago describes a fluid layer confined between two horizontal boundaries, which is heated from below. ${ }^{285} \mathrm{~A}$ hexagonal cellular pattern is one of the typical resultant patterns of convection. Similar to the thermal convection, many spatial patterns can also form by electro-hydrodynamic convection in electrochemical processes with an electric potential gradient in a charged liquid under isothermal conditions. ${ }^{286-288}$ Such convection was found to be driven by an electric field volume force exerted on the uncompensated excess charge presented 
in the fluid, the local value of which is related to the spatial distribution of the electric field through Poisson's equation. ${ }^{289}$

Electric force can drive electro-hydrodynamic convective flows to form various self-organised patterns in a micro or millimeter scale. ${ }^{290.291}$ Whether such an electro-hydrodynamic convection process can be found in a nanoscale system as in the formation of nanoporous AAO is of great interest. In the present work, we found that an ionic nanoconvection could form in the vicinity of the oxide electrolyte (O/E) interface (Figure 6-2), leading to the commencement of pore ordering during the early stage of anodisation. This ionic nanoconvection model reproduces the linear dependency of inter-pore (pore centre to pore centre) distance on applied voltage, which is known from previous experiments but has not been well interpreted theoretically or numerically. ${ }^{70}$

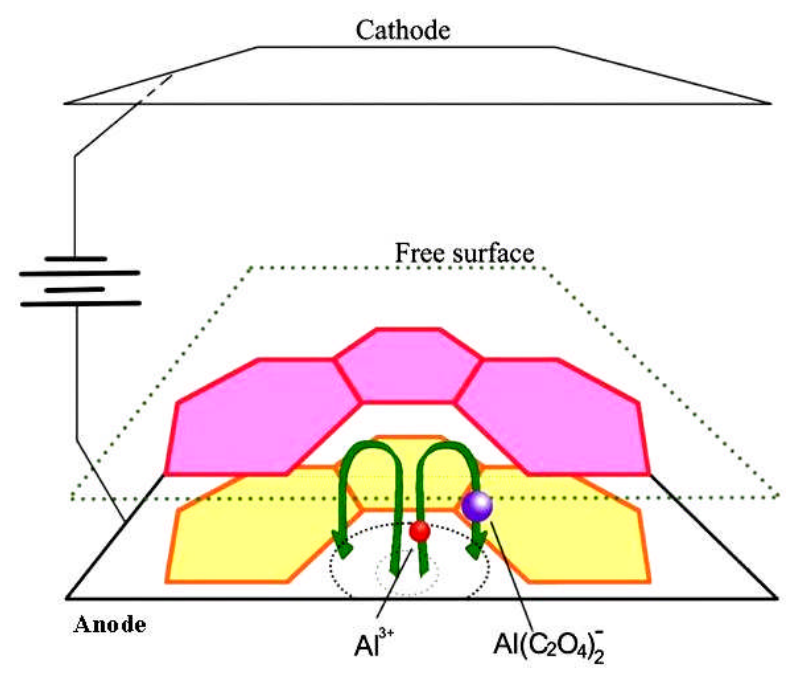

Figure 6-2. An nanoconvection model driven by electrostatic force near the O/E interface. The flux of electrolyte and the ions are shown schematically together with arrangement of honeycomb like AAO pores.

The overall reaction at the $\mathrm{O} / \mathrm{E}$ interface during the anodisation of aluminium metal can be written as:

$$
\mathrm{Al}_{2} \mathrm{O}_{3}+n \mathrm{H}_{2} \mathrm{O} \rightarrow 2 \mathrm{Al}^{3+}+(3+n-x) \mathrm{O}^{2-}+x O \mathrm{H}^{-}+(2 n-x) \mathrm{H}^{+}
$$


where $\mathrm{n}$ is specially introduced to indicate the ratio of the dissociation of water to the dissolution of $\mathrm{Al}_{2} \mathrm{O}_{3}$. It is assumed all the anions produced from eq. (6.1) migrate in the electric field from the solid surface to the oxide/metal interface, contributing to the formation of $\mathrm{Al}_{2} \mathrm{O}_{3}$. Some $\mathrm{Al}^{3+}$ cations are ejected into the electrolyte without forming oxide. It is estimated that under a mild anodisation condition, $14.4 \%$ of the total $\mathrm{Al}^{3+}$ cations moving to the electrolyte are from the dissolution of $\mathrm{Al}_{2} \mathrm{O}_{3}$ at the $\mathrm{O} / \mathrm{E}$ interface and $85.6 \%$ of $\mathrm{Al}^{3+}$ are from the oxide/metal interface. $^{78,225,249}$ The ejection of these $\mathrm{Al}^{3+}$ into the electrolyte may induce an ionic convection near the $\mathrm{O} / \mathrm{E}$ interface.

For an electrochemical cell, the classic Nernst theory assumes a thin static liquid layer adjacent to the electrode. This has been proved to be incorrect, as local convection may occur in this layer with the convection size and pattern determined by the local space charge distribution. ${ }^{292,293}$ From another perspective Pundik et al ${ }^{294}$ showed that periodically distributed small vortexes could be produced on the local spatial periodicity of surface perturbation, and the size of these vortices could increase with time as long as they are smaller than the cell size. Accordingly, the number of vortices would decrease, which is in accordance with the observation by Rapaport et $a l^{295}$ Their work on the molecular dynamics simulation of pattern formation in three-dimensional Rayleigh-Benard thermal convection showed an initially disordered set of small convection cells that grew, merged, and rearranged, culminating in what appeared to be a stable array of hexagonal cells. In the case of electro-hydrodynamics, convection cells are driven by an electric field exerted on excess charges presented in the fluid. The charge density is neutral in the bulk of the electrolyte except in a diffusion layer close to the electrode. Consequently, for modeling the convection in an electrochemical cell, we need only to consider the diffusion layer. In the present work, an ionic convection model is proposed to elucidate the ionic movement near the $\mathrm{O} / \mathrm{E}$ interface of AAO during the anodisation.

It is believed that, in the initial stage of anodisation, convection with a scale of several nanometers could be developed at the $\mathrm{O} / \mathrm{E}$ interface. The dimension of the convection cells gradually increases, but is confined by the thickness of the diffusion layer. 


\subsection{Details of the theoretical simulation of the ionic convection}

The theoretical simulation part of this ionic convection model was performed in a collaboration with Mr Shijing Lu from department of physics of Zhejiang University in China. To simplify the calculation, only the steady-state convection with a dimension fitting to the space charge diffusion layer was considered in the simulation. In addition we assumed that the ions move together with the flow of electrolyte. The properties of the fluid (density, viscosity) were supposed to be constant. Based on these considerations a set of corresponding equations can be written as follows:

$$
\begin{aligned}
& \frac{\partial v}{\partial t}+(v \cdot \nabla) v=-\frac{1}{\rho} \nabla P+\eta \nabla v-\frac{\sigma}{\rho} \nabla \varphi \\
& \frac{\partial \sigma_{p}}{\partial t}+(v \cdot \nabla) \sigma_{p}=\kappa \nabla^{2} \sigma_{p}-\gamma \sigma_{p} \\
& \frac{\partial \sigma_{n}}{\partial t}+(v \cdot \nabla) \sigma_{n}=\kappa \nabla^{2} \sigma_{n}+\gamma \sigma_{p} \\
& \nabla^{2} \varphi=-\frac{\sigma}{\varepsilon} \\
& \nabla^{2} v=0
\end{aligned}
$$

where $\mathrm{v}(\mathrm{m} / \mathrm{s})$ is the velocity of electrolyte, $\rho\left(\mathrm{kg} / \mathrm{m}^{3}\right)$ is the density of electrolyte, $\mathrm{P}(\mathrm{Pa})$ is pressure evolving in the fluid, $\eta\left(\mathrm{m}^{2} / \mathrm{s}\right)$ is the kinematics viscosity, $\sigma_{\mathrm{n}}, \sigma_{\mathrm{p}}, \sigma=\sigma_{\mathrm{p}}-\sigma_{\mathrm{n}}\left(\mathrm{C} / \mathrm{m}^{3}\right)$ are separate densities of negative, positive and total space charges. $\varphi(\mathrm{V})$ is the electric potential. $\kappa$ $\left(\mathrm{m}^{2} / \mathrm{s}\right)$ is the diffusivity. $\varepsilon\left(\mathrm{C}^{2} / \mathrm{N} \cdot \mathrm{m}^{2}\right)$ is dielectric constant of water. The eq. (6.2), modified from the Navier-Stokes equation, ${ }^{297}$ is used to describe the Raleigh-Benard convection by replacing the buoyant force item with an electric force item. Eqs. (6.3) and (6.4) are diffusion equations for anions and cations separately. Eqs. (6.5) and (6.6) are the Poisson's equations for electrostatics and incompressible fluids, respectively.

Both eqs. (6.3) and (6.4) have a shield and enhance item $\gamma \sigma_{\mathrm{p}}$. We used this item to measure the polarization effect. With this effect, negative charge will be enhanced and positive charge will be shielded when $\mathrm{Al}^{3+}$ cations are coordinated by $\left(\mathrm{C}_{2} \mathrm{O}_{4}\right)^{2-}$ anions to form some complex ions, like $\mathrm{Al}\left(\mathrm{C}_{2} \mathrm{O}_{4}\right)^{+}, \mathrm{Al}\left(\mathrm{C}_{2} \mathrm{O}_{4}\right)_{2}{ }^{-}$, etc., when $\mathrm{Al}^{3+}$ is dissolved in the electrolyte. 
We set up specific boundary conditions by considering the following effects:

(1) The electrolyte has non-slip boundary conditions on the surface of the O/E interface due to the viscidity of electrolyte and has periodic boundary conditions on the two sides of the solving region. For simplicity we assume zero flux on the free surface of the convective cell since the average flux of electrolyte on the surface is zero, specifically: $\left.v\right|_{b 1, b 3}=0$ and $\left.\nabla_{n} v\right|_{b 2}=0$. Here b1 is the free surface of the convective cells with a distance $\lambda$ from the $\mathrm{O} / \mathrm{E}$ interface, $\mathrm{b} 3$ refers to the surface of the O/E interface and b2 refers to the two sides of convective vortexes in a 2D model.

(2) As a boundary condition, the positive space charge density has a zero value on the free surface because the electrolyte beyond this surface is neutral and has constant flux on the O/E interface. The negative space charge density, on the other hand, has a zero value on the O/E interface and zero flux on the free surface. Both positive and negative space charge densities have symmetric boundary conditions on the vortex wall. Accordingly, $\left.\nabla_{n} \sigma_{p}\right|_{b 3}=-f_{0},\left.\nabla_{n} \sigma_{p}\right|_{b 2}=0$, $\left.\sigma_{p}\right|_{b 1}=0,\left.\nabla_{n} \sigma_{n}\right|_{b 1}=0,\left.\nabla_{n} \sigma\right|_{b 2}=0$ and $\left.\sigma_{n}\right|_{b 3}=0$. Here $f_{0}$ determines the flux of positive charges, whose equivalent in physics is charge density.

(3) The electric field boundary conditions were set as follows: zero electric potential at the free surface, constant electric potential with a value decided by the anodising voltage. i.e. $\left.\varphi\right|_{b 1}=0$, $\left.\nabla_{n} \varphi\right|_{b 2}=0,\left.\varphi\right|_{b 3}=c V_{\text {anodizing }}$ (c is a prefactor)

\subsection{Results of the ionic convection simulation}

We integrated the governing eqs. (6.2) - (6.6) using a professional finite element analysis package FlexPDE 5.0 (http://www.pdesolutions.com). A useful but not unique convention of expressing time, length and mass in units of $\lambda^{2} / \eta, \lambda$ and $\rho \lambda^{3}$ was used to lower the computation error. It is not surprising that the governing equations may lead to many turbulent solutions if $\lambda$ is inappropriately chosen. In conventional Rayleigh-Benard convection theory, it is convenient to select proper convection layer by selecting a proper Rayleigh number $R_{a}\left(R_{a} \propto \lambda^{3}\right)$. In our model, we have more complex nonlinear items which make Rayleigh number analysis no longer valid. To find proper solving region, we simply vary the diffusion thickness to find valid regions for $\lambda$ 
which maintains stable convection patterns. The $\lambda$ value corresponding to the largest convection cell is taken as the best approximation for thickness of diffusion layer. The associated inter-cell distance in convection is recorded as the value for inter-pore distance $\left(D_{\text {int }}\right)$. Specifically with a given anodising voltage of $30 \mathrm{~V}$, anodisation of aluminium in $0.3 \mathrm{M}$ oxalic acid, the flux of positive charge is about $2.9 \mathrm{~mA} / \mathrm{cm}^{2}$ in conformity with experimental conditions. ${ }^{78,225,249}$ The cell dimension is about $75 \mathrm{~nm}$ with a voltage of $30 \mathrm{~V}$ according to the experimental observation. ${ }^{70} \mathrm{~A}$ value of $\lambda$ of about $50 \mathrm{~nm}$ is reasonable, as it is sufficiently larger than half of the average cell size in AAO. We integrated the governing equations in a two dimensional rectangular region under the above anodic parameters. It was found that in order to obtain a solution for a stable convective pattern, the potential drop across the convective layer should have a value of $0.3 \mathrm{~V}$, corresponding to about one hundredth of the anodising voltage.

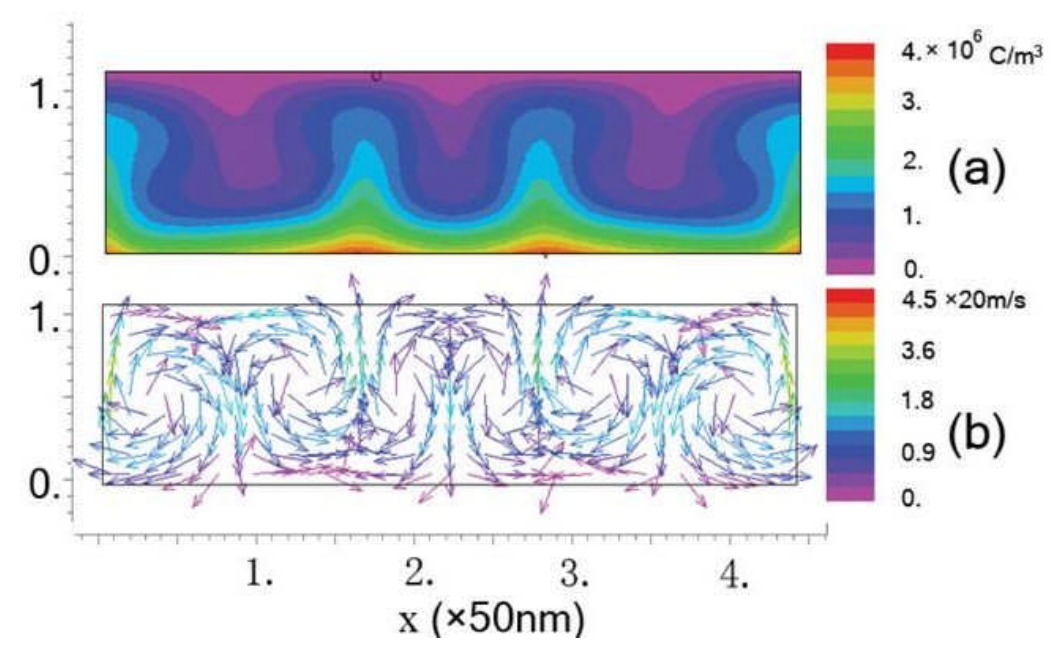

Figure 6-3. A calculated nanoconvectional pattern in an anodisation process of Al. A numerical solution in a solving area $[0,240 \mathrm{~nm}] \times[0,50 \mathrm{~nm}]$ shows a natural convectional pattern with anodising voltage of $30 \mathrm{~V}$ and kinematics viscosity of $1.0 \times 10^{-6} \mathrm{~m}^{2} / \mathrm{s}$. The density of electrolyte was assumed to be $1.0 \mathrm{~kg} / \mathrm{m}^{3}$. The ionic diffusivity was $1.2 \times 10^{-9} \mathrm{~m}^{2} / \mathrm{s}$. (a) Contour plot of density of cations mainly $\mathrm{Al}^{3+}$ and $\mathrm{H}^{+}$. (b) Vector plot of vortices.

Figure 6-3 shows density distribution of the cations. Near the O/E interface, cations gather at the positions of $0,85,140,220 \mathrm{~nm}$ where the cations, driven by electric force, flow away from $\mathrm{O} / \mathrm{E}$ interface as demonstrated in Figure 6-3b, corresponding to the flow-up site in Figure 6-2. 
The high concentration of the cations results in a low concentration of anions including $\mathrm{O}^{2-}$ and $\mathrm{OH}^{-}$, therefore, a relatively lower probability to form aluminium oxide. On the other hand, as cations depart are ejected from the oxide surface, anions migrate towards the opposite direction.

Therefore, the $\mathrm{pH}$ value in the flow-down area is significantly higher than that in the flow-up area, resulting in periodical variation of the dissolution rate of $\mathrm{Al}_{2} \mathrm{O}_{3}$ on the oxide surface. The consequence of the faster dissolution rate at the flow-up sites leads to the formation of pores. Following this reasoning we are able to determine the inter-pore distance by measuring the average distance between two convection cells. As in the above case, an average convective size of $73.3 \mathrm{~nm}$ could be obtained which is in a good agreement with the $75 \mathrm{~nm}$ indicated by a previous report. ${ }^{70}$

Supposing that the solution domain has a fixed ratio of the height to length for a stable convection, simulation of the convection patterns under different potentials allows us to reveal the dependence of inter-pore distance on the anodising voltage. Assuming that the potential drop across the convective layer has a constant ratio to the anodising voltage, the prefactor $\mathrm{c}$ is constant, the cell sizes of the stable convective patterns obtained show a linear relation with the anodising voltage, $D_{i n t}=2.64 U-0.15$, as shown in Figure 6-4, when the current density is determined by $j=1.8 \times \exp (0.0256 U)$ as established previously. ${ }^{225}$ When the current density of anodisation is kept a fixed value of $5 \mathrm{~mA} / \mathrm{cm}^{2}$, the simulation with anodising voltage ranging from $10 \mathrm{~V}$ to $110 \mathrm{~V}$ shows a very similar linear relation between the cell size and anodising voltage, $D_{i n t}=2.65 U-0.15$. This is in accordance with the previous reports. ${ }^{24,70,297}$ Consequently, a small fluctuation of current density has little impact on the cell size of AAO. Experimentally, in fact, a similar relation between the cell size and anodising voltage, $D_{\text {int }} \approx 2.5 U \mathrm{~nm} / \mathrm{v}$, has been found in AAO formation in different electrolytes with different current density dependence on anodising voltage, although the relation was not well interpreted theoretically or numerically. ${ }^{70}$ In the present work, the newly proposed ionic nanoconvection model has produced this linear relationship, indicating that the voltage effect on the inter-pore distance may exist at a very early stage of anodisation.

The above calculation is in two dimensions (2D). It is quite safe to generalize the $2 \mathrm{D}$ results to $3 \mathrm{D}$ according to mathematical analysis of pattern selection by Golubitsky et $a l^{298}$ where they proved that parallel rolls and hexagonal cells are two competitive patterns in convection systems, 
supported by other reports, ${ }^{299}$ including that by Chazalviel, in which the author found a honeycomb convection pattern with an average cell size comparable to the diffusion length in the thin-layer electroconvection experiments. ${ }^{300}$

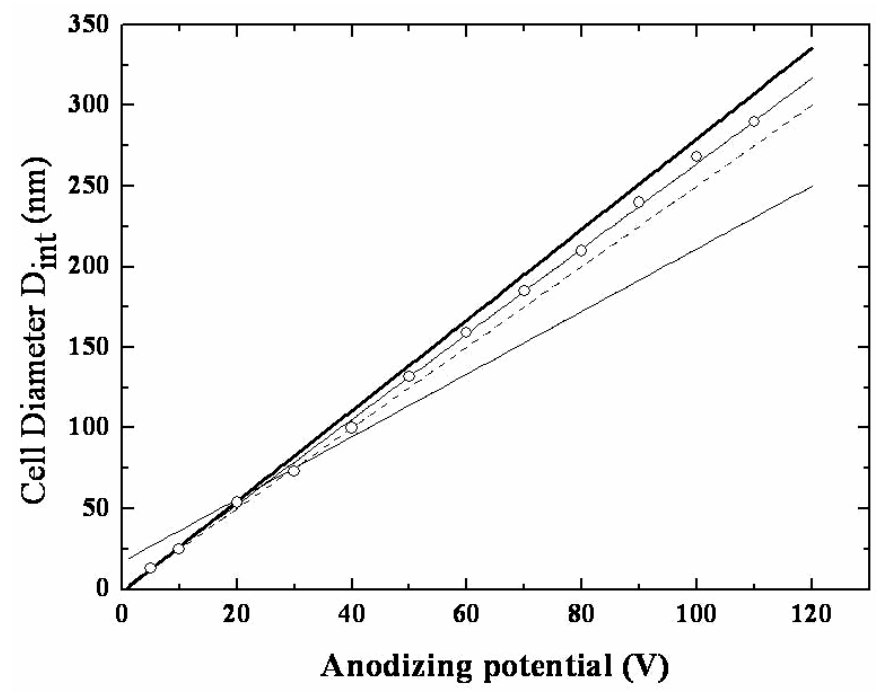

Figure 6-4. Relationship of the cell diameter $\left(\mathrm{D}_{\text {int }}\right)$ and the anodizing potential $(\mathrm{V})$. Data from the numerical results of our model are plotted in circles, fitting (solid line) to a formula of $D_{\text {int }}=$ $2.64 \mathrm{U}-0.15$, while data from references are plotted in thin solid line, ${ }^{24}$ dash-dot line ${ }^{70}$ and thick line respectively. ${ }^{297}$

In summary, the ionic nanoconvection driven by the electric force exerted on the space charge layer in the vicinity of electrolyte/oxide interface is established to be the main driving force of the pore ordering at the early stage of the anodisation. 


\section{Conclusions and Future Work}

Anodic metal oxides have diverse applications in the prevention of corrosion of metal substrates from their service environment, forming capacitor dielectrics, templating nanomaterials and in many other fields such as in sensing, in photocleavage of water and in dye-sensitized solar cells. An ionic convection model and equifield strength model have been established, accounting for the self-organisation of highly ordered porous anodic metal oxide films. The determined factors of the anodic films, pore size and porosity, are studied which will help to control the structures of the grown anodic films more precisely.

\subsection{Conclusion}

Hexagonal patterned $\mathrm{Al}_{2} \mathrm{O}_{3}$ nanopore arrays and $\mathrm{TiO}_{2}$ nanotubular arrays have been achieved by anodisation of corresponding metal substrates in proper electrolytes. The two characteristic microstructural features of anodic aluminium oxide (AAO) and anodic titanium oxide (ATO) were investigated using SEM and HRTEM. The observations of the hemispherical electrolyte/oxide and oxide/metal interfaces, uniform thickness of the oxide layer, as well as self-adjustment of the pore size and pore ordering can be well explained by the newly proposed equifield strength model.

It was established that, in the initial stage of anodisation, the ionic nanoconvection driven by the electric force exerted on the space charge layer in the vicinity of the electrolyte/oxide interface could lead to the primary pore ordering at the early stage of the anodisation.

The fundamental principle of the equifield strength model was the equilibrium between the electric field driven oxidation rate of the metal and electric field enhanced dissolution rate of oxide. This model allows us to understand the formation mechanism of porous anodic metal oxide films, including the formation of the spherical segment morphology of the pore bottom, the growth of a single pore, and the self-organisation of pore arrays. It has been found that the electric field enhanced dissociation of water is extremely important in determination of the porosity of anodic metal oxide. The dissociation rate of water for supplying oxygen anions to the oxide/metal interface is the most important driving force for adjusting the porosity of AAO, or 
the ratio of the pore size to the cell dimension. The porosity of AAO and ATO films was found to be governed by the relative dissociation rate of water which is dependent on anodisation conditions, such as electrolyte, applied voltage, current density and electric field strength. Using an empirical method, the relations between the porosity of the anodic films and the anodisation parameters, such as electric field strength, current density and applied voltage, have been established.

When ATO nanotubes were examined by using TEM, it was found that the wall of the nanotubes consists of two layers. The inner layer was titania and the outer layer was titanium hydroxide, implying that $\mathrm{OH}^{-}$anions can move from the electrolyte/oxide interface to the oxide/metal interface to form a titanium hydroxide layer, although the exact formula could not be determined. It was assumed that the hydroxide layer decomposed continuously into oxide during the anodisation since its thickness at the nanotube bottom maintains constant, resulting a volume shrinkage of the tube wall. An aluminium hydroxide layer was never observed in AAO, since even if it formed, it would decompose quickly due to its very low decomposition energy. That can explain why ATO nanotubular arrays are obtained by titanium anodisation while the aluminium anodisation producing AAO nanopore arrays.

Quantum-chemical model computations confirmed that an external electric field can facilitate the heterolytic dissociation of properly oriented water molecules significantly. This fundamental finding strongly supports the supply of the oxygen component during the anodic oxidation of valve metal via electric field enhanced heterolytic dissociation of water at the electrolyte/oxide interface. Assuming that suitable orientations of water molecules are brought about by adsorption at the oxide surface, and employing physically reasonable relations between macroscopic and local electric field strengths, a remarkably good accord is found between key quantities derived from the quantum-chemical models and the experimental data.

\subsection{Future work}

During the oxidation of aluminium to alumina, a volume expansion is generated accompanied by the formation and relaxation of mechanical stress. Beside, as discussed by previous authors, the high plasticity of alumina could result in a viscous flow during the formation of AAO, which 
might be account for the pore ordering formation. In the future, we would use tungsten as a diacritical species in tracer study of anodisation process to confirm and measure the expansion during anodic oxidation of aluminium metal, and to investigate the exact movement of the produced oxide and the influence of the viscous flow on the pore formation or even pore ordering.

Based on the established equifield strength model, a predicted theoretical curve for pore diameters of AAO, prepared in $0.3 \mathrm{M}$ oxalic acid at room temperature of $15{ }^{0} \mathrm{C}$, versus anodising voltage show both a minimized peak and a maximal peak together. Detailed experimental data is needed in the future to verify the theoretical prediction on the dependence of AAO pore diameter on the anodising voltage.

We have proposed the ionic convection model to explain the initial ordering at the early stage of anodisation of AAO. A convinced experimental validation needs to be done in the future. The magnetic field effect on the ionic convection will be studied also.

AAO prepared under high current density and high voltage has exhibited interesting morphologies such as tube structure and bamboo like corrosion pattern. The formation of AAO under high voltage in novel electrolytes and the growth mechanism of the interesting morphologies will be carried out in the future.

We have showed that the O-ring structure and the most outer surface of the ATO which connecting neighboring tubes could be annealed to single crystalline phase, though the bulk part remained polycrystalline. It is expected that further annealing may allow recrystallisation expanding from the outer surface to the inner surface via an Ostwald ripening process and eventually form a connected single crystal nanotubular array. A reasonably low temperature will be carefully chosen and long crystallisation time will be applied, in order to facilitate the single crystallisation which start in the outer hydroxide layer and finally expand to the inner oxide layer.

ATO films prepared under high voltage, above $100 \mathrm{~V}$, show a cleaner surface than those prepared under lower voltage. Also more small crystals were found in the as prepared ATO tube walls. The difference between the high voltage and low voltage anodisation of titanium, as well as the influence of electrolytes will be studied in the future.

As the formation of a highly ordered ATO nanotube array usually needs a long anodisation for 
adjustment, a lot of work need to be carried out to produce ordered ATO arrays on a thin titanium film.

Though AAO and ATO films have widely application in scientific researches and in industry, in this dissertation, we focus on the more fundamental problems especially in the formation mechanism of the self organisation of the anodic oxide films. In the future we will carry out the application researches for AAO nanochannels and ATO nanotubes.

AAO as a template for the formation of nanostructure materials has widely application. Target metal, metal oxide, alloy, and heterojunctions, nanowires and nanotubes could be formed using the AAO template via chemical vapor deposition or electrochemical deposition methods. The selection of electrolytes and the anodisation parameters needs to be optimized to achieve preset aims.

With a better control of the structure and morphology of the produced ATO nanotubes, length, diameters and uniformity, we are aiming to achieve a dye sensitized solar cell based on the anodic $\mathrm{TiO}_{2}$ nanotubes with higher photo energy conversion rate. Doping of ATO nanotubes will also be carried out to increase their photocatalyst activity in organics decomposition and water photolysis. 


\section{References}

1 A. Hagfeldt and M. Gratzel, Chem. Rev., 1995, 95, 49.

2 C. G. Granqvist, 'Handbook of Inorganic Electrochromic Materials', Elsevier Science, Amsterdam, 1995.

3 F. L. Boca Raton, 'Handbook of Biosensors and Electronic Noses', CRC Press, 1997.

4 A. Manthiram and J. Kim, Chem. Mater., 1998, 10, 2895.

5 S. Sarangapani, B. V. Tilak and C. P. Chen, J. Electrochem. Soc., 1996, 143, 3791.

6 J. H. Fendler, Chem. Mater., 1996, 8, 1616.

7 J. F. Fernandez-Bertran, Pure Appl. Chem., 1999, 71, 581.

8 A. Fini and A. Breccia, Pure Appl. Chem., 1999, 71, 573.

9 Y. Xia, J. A. Rogers, K. E. Paul and G. M. Whitesides, Chem. Rev., 1999, 99, 1823.

10 C. R. Martin, Chem. Mater., 1996, 8, 1739.

11 G. D. Bengough and J. M. Stuart, Brit. Patent, 1923, 223994.

12 A. C. Jason and J. L. Wood, Proc. Phys. Soc. B, 1955, 68, 1105.

13 C. J. Dell'Oka, D. L. Pulfrey, L.Young, 'Physics of thin films', Academic Press, New York, 1971.

14 M. Nakao, S. Oku, T. Tamamura, K. Yasui and H. Masuda, Jpn. J. Appl. Phys., 1999, 38, 1052.

15 J. Liang, H. Chik, A. Yin and J. Xu, J. Appl. Phys., 2002, 91, 2544.

16 S. Shingubara, O. Okino, Y. Murakami, H. Sakaue and T. Takahagi, J. Vac. Sci. Technol., 2001, B19, 1901.

17 J. Li, C. Papadopoulos and J. M. Xu, Appl. Phys. Lett., 1999, 75, 367.

18 J. Li, C. Papadopoulos and J. Xu, Nature, 1999, 402, 253.

19 W. Hu, D. Gong, Z. Chen, L. Yuan, K. Saito, C.A. Grimes and P. Kichambare, Appl. Phys. Lett., 2001, 79, 3083.

20 S. Basu, S. Chatterjee, M. Saha, S. Bandyopadhay, K. K. Mistry and K. Sengupta, Sens. Actuat. B: Chem., 2001, 79, 182.

21 B. C. Yang, M. Uchida, H. M. Kim, X. D. Zhang, and T. Kokubo, Biomaterials, 2004, 25, 1003. 
22 G. K. Mor, K. Shankar, M. Paulose, O. K. Varghese and C.A. Grimes, Nano. Lett., 2005, 5, 191.

23 G. K. Mor, K. Shankar, M. Paulose, O. K. Varghese and C.A. Grimes, Nano. Lett., 2006, 6, 215 .

24 F. Keller, M. S. Hunter and D. L. Robinson, J. Electrochem. Soc., 1953, 100, 411.

25 J. P. O’Sullivan and G. C. Wood, Proc. R. Soc. Lond. A, 1970, 317, 511.

26 G. E. Thompson, R. C. Furneaux, G. C. Wood, J. A. Richardson and J. S. Goode, Nature, 1978, 272, 433.

27 O. Jessensky, F. Müller and U. Gösele, Appl. Phys. Lett., 1998, 72, 1173.

28 D. W. Gong, C. A. Grimes, O. K. Varghese, W. C. Hu, R. S. Singh, Z. Chen and E. C. Dickey, J. Mater. Res., 2001, 16, 3331.

29 J. M. Macak, H. Tsuchiya and P. Schmuki, Angew. Chem. Int. Ed., 2005, 44, 2100.

30 M. Paulose, K. Shankar, S. Yoriya, H. E. Prakasam, O. K. Vargh- ese, G. K. Mor, T. A. Latempa, A. Fitzgerald and C. A. Grimes, J. Phys. Chem. B, 2006, 110, 16179.

31 H. Tsuchiya and P. Schmuki, Electrochem. Commun., 2005, 7, 49.

32 I. V. Sieber, H. Hildebrand, A. Friedrich and P. Schmuki, Electrochem. Commun., 2005, 7, 97.

33 I. V. Sieber, B. Kannan and P. Schmuki, Electrochem. Solid-State. Lett., 2005, 8, J10.

34 N. Mukherjee, M. Paulose, O. K. Varghese, G. K. Mor and C. A. Grimes, J. Mater. Res., 2003, 18, 2296.

35 G. B. Stefanovich, A. L. Pergament, A. A. Velichko and L. A. Stefanovich, J. Phys: Condens. Matter., 2004, 16, 4013.

36 H. Tsuchiya, J. M. Macak, I. Sieber, L. Taveira and P. Schmuki, Small, 2005, 1, 722.

37 T. P. Hoar and N. F. Mott, J. Phys. Chem. Solids, 1959, 9, 97.

38 K. V. Heber, Electrochim. Acta, 1978, 23, 127.

39 G. E. Thompson and G. C. Wood, 'Anodic films on aluminium', Academic Press, New York, 1983.

40 H. Masuda and K. Fukuda, Science, 1995, 268, 1466.

41 H. Masuda, M. Yosuya and M. Ishida, Jpn. J. Appl. Phys., 1998, 37, 1090. 
42 J. M. Macak, H. Tsuchiya, L. V. Taveira, S. Aldabergerova and P. Schmuki, Angew. Chem. Int. Ed., 2005, 44, 7463.

43 P. G. Sheasby and R. Pinner, 'The surface treatment and finishing of aluminium and its alloys', ASM International \& Finishing Publications, 2001.

44 A. Guntherschulze and H. Betz, Z. Phys., 1931, 68, 145.

45 A. Guntherschulze and H. Betz, Z. Phys., 1934, 92, 367.

46 J. Siejka and C. Ortega, J. Electrochem. Soc., 1977, 124, 883.

47 J. W. Diggle, T. C. Downie and C. W. Goulding, Chem. Rev., 1969, 69, 365.

48 F. Y. Li, L. Zhang and R. M. Metzger, Chem. Mater., 1998, 10, 2470.

49 R. C. Furneaux, G. E. Thompson and G. C. Wood, Corros. Sci., 1978, $18,853$.

50 G. E. Thompson, Y. Xu, P. Skeldon, K. Shimizu, S. H. Han and G. C. Wood, Philos. Mag. B, 1987, 55, 651 .

51 F. Brown and W. D. Mackintosh, J. Electrochem. Soc., 1973 ,120, 1096.

52 P. Skeldon, G. E. Thompson, S. J. Garcia-Vergara, L. Iglesias-Rubianes and C. E. Blanco-Pinzon, Electrochem. Solid-State Lett., 2006, 9, B47.

53 J. A. Davies, B. Domeij, J. P. S. Pringle and F. Brown, J. Electrochem. Soc., 1965, 112, 675.

54 Y. Xu, G. E. Thompson and G. C. Wood, Trans. Inst. Met. Finish, 1985, 63, 98.

55 K. Shimizu, K. Kobayashi, G. E. Thompson, and G. C. Wood, Philos. Mag. A, 1992, 66, 643.

56 G. Hass, J. Opt. Soc. Amer., 1949, 39, 532.

57 A. Charlesby, Proc. Phys. Soc., 1953, 866, 317.

58 S. Setoh and A. Miyata, Sci. Pap. Znst. Phys. Chem. Res. (Tokyo), 1932, 19, 237.

59 C. J. L. Booker, J. L. Wood and A. Walsh, Nature, 1955, 176, 222.

60 C. J. L. Booker, J. L. Wood and A. Walsh, Brit. J. Appl. Phys., 1957, 8, 347.

61 M. Paganelli, Aluminio, 1958, 27, 3.

62 H. Grubitsch, W. Geymeyer and E. Buvik, Aluminium, 1961, 37, 569.

63 R. L. Burwell, P. A. Smudski and T. P. May, J. Amer. Chem. Soc., 1947, 69, 1525.

64 G. Paolini, M. Masaero, F. Sacchi and M. Paganelli, J. Electrochem. Soc., 1965, 112, 32.

65 R. C. Plumb, J. Electrochem. Soc., 1958, 105, 498.

66 G. A. Dorsey Jr, J. Electrochem. Soc., 1966, 113, 169.

67 G. E. Thompson and G. C. Wood, Nature, 1981, 290, 230. 
68 H. Masuda, H. Yamada, M. Satoh, H. Asoh, M. Nakao and T. Tamamura, Appl. Phys. Lett., 1997, 71, 2770.

69 S. Bandyopadhyay, A. E. Miller, H. C. Chang, G. Banerjee, V. Yuzhakov, D. F Yue, R. E.

Ricker, S. Jones, J. A. Eastman, E. Baugher and M Chandrasekhar, Nanotech., 1996, 7, 360.

70 W. Lee, R. Ji, U. Gösele and K. Nielsch, Nat. Mater., 2006, 5, 741.

71 S. Shingubara, J. Nanoparticle Res., 2003, 5, 17.

72 S. L. Sung, S. H. Tsai, C. H. Tseng, X. W. Liu and H. C. Shih, Appl. Phys. Lett., 1999, 74, 197.

73 M. Sun, G. Zangari, M. Shamsuzzoha and R. M. Metzger, Appl. Phys. Lett., 2001, 78, 2964.

74 Z. X. Su, J. Sha, G. W. Pan, J. X. Liu, D. R. Yang, C. Dickinson and W. Z. Zhou, J. Phys. Chem. B, 2006, 110, 1229.

75 L. Ba and W. S. Li, J. Phys. D: Appl. Phys., 2000, 33, 2527

76 G. K. Singh, A. A. Golovin and I. S. Aranson, Phys. Rev. B, 2006, 73, 205422.

77 A. I. Vorobyova, E. A. Outkina and A. A. Khodin, Rus. Microelectr., 2007, 36, 384.

78 Z. X. Su and W. Z. Zhou, Adv. Mater., 2008, 20, 3663.

79 A. Despica and V. P. Parkhutik, 'Electrochemistry of aluminium in aqueous solutions and physics of its anodic oxide', Plenum Press, New York, 1989.

80 T. Valand and K. E Heusler, J. Electroanal. Chem., 1983, 149, 71.

81 G. C.Wood, P. Skeldon, G. E. Thompson and K. Shimizu, J. Elecrrochem. Soc., 1996, 43, 74.

82 S. J. Garcia-Vergara, L. Iglesias-Rubianes, C. E. Blanco-Pinzon, P. Skeldon, G. E. Thompson and P. Campestrini, Proc. R. Soc. A, 2006, 462, 2345.

83 H. Masuda and M. Satoh, Jpn. J. Appl. Phys., 1996, 35, L126.

84 K. Nielsch, J. Choi, K. Schwirn, R. B. Wehrspohn and U. Gösele, Nano Lett., 2002, 2, 677.

85 J. O. Bockris and A. K.N. Reddy, 'Modern electrochemistry', Plenum Press, New York, 1970.

86 A. R. Despic and V. P. Parkhutik, 'Modern aspects of electrochemistry', Plenum Press, New York, 1989.

87 V. V.Yuzhakov, H. C. Chang, and A. E. Miller, Phys. Rev. B, 1997, 56, 12608.

88 P. Skeldon, K. Shimizu, G. E. Thompson and G. C. Wood, Surf. Interface Anal., 1983, 5, 247. 89 A. P. Li, F. Muller, A. Birner, K. Nielsch and U. Gösele, J. Appl. Phys., 1998, 84, 6023. 
90 V. P. Parkhutik and V. I. Shershulsky, J. Phys. D: Appl. Phys., 1992, 25, 1258.

91 S. Z. Chu, K. Wada, S. Inoue, M. Isogai and A. Yasumori, Adv. Mater., 2005, 17, 2115.

92 H. Masuda, K. Yada and A. Osaka, Jpn. J. Appl. Phys., 1998, 37, L1340.

93 S. Ono, M. Saito, M. Ishiguro and H. Asoh, J. Electrochem. Soc., 2004, 151, B473.

94 S. Ono, M. Saito and H. Asoh, Electrochem. Solid-State Lett., 2004, 7, B21.

95 G. A. Ozin and A. C. Arsenault, Nanochemistry: A Chemical Approach to Nanomaterials, Royal Society of Chemistry, Cambridge, 2005.

96 G. Hodes, Electrochemistry of Nanomaterials, Wiley-VCH, Weinheim, Germany, 2001.

97 H. H. Ou and S. L. Lo, Sep. Purif. Technol., 2007, 58, 179.

98 X. Chen and S. S. Mao, Chem. Rev., 2007, 107, 2891.

99 D. V. Bavykin, J. M. Friedrich and F. C. Walsh, Adv. Mater., 2006, 18, 2807.

100 M. Adachi, Y. Murata, M. Harada and S. Yoshikawa, Chem. Lett., 2000, 29, 942.

101 S. Z. Chu, S. Inoue, K. Wada, D. Li, H. Haneda and S. Awatsu, J. Phys. Chem. B, 2003, $107,6586$.

102 O. K. Varghese, D. Gong, M. Paulose, K.G. Ong, E.C. Dickey and C. A. Grimes, Adv. Mater., 2003, 15, 624.

103 G. K. Mor, M. A. Carvalho, O. K. Varghese, M. V. Pishko and C. A. Grimes, J. Mater. Res., 2004, 19, 628.

104 O. K. Varghese, G. K. Mor, C. A. Grimes, M. Paulose and N. Mukherjee, J. Nanosci. Nanotech., 2004, 4, 733.

105 M. Paulose, O. K. Varghese, G. K. Mor, C. A. Grimes and K. G. Ong, Nanotech., 2006, 17, 398.

106 G. K. Mor, K. Shankar, O. K. Varghese, and C. A. Grimes, J. Mater. Res., 2004, 19, 2989.

107 O. K. Varghese, M. Paulose, K. Shankar, G. K. Mor and C. A. Grimes, J. Nanosci. Nanotech., 2005, 5, 1158.

108 S. Uchida, R. Chiba, M. Tomiha, N. Masaki and M. Shirai, Electrochem., 2002, 70, 418.

109 M. Adachi, Y. Murata, I. Okada, and S. Yoshikawa, J. Electrochem. Soc., 2003, 150, G488.

110 M. Paulose, K. Shankar, O. K. Varghese, G. K. Mor, B. Hardin and C. A. Grimes, Nanotech., 2006, 17, 1446.

111 C. K. Dyer and J. S. L. Leach, J. Electrochem. Soc., 1978, 125, 1032. 
112 J. W. Schultze, M. M. Lohrengel and D. Ross, Electrochim. Acta, 1983, 28, 973.

113 P. Schmuki, J. Solid State Electrochem., 2002, 6, 145.

114 J. C. Marchenoir, J. P. Loup and J. Masson, Thin Solid Films, 1980, 66, 357.

115 L. Arsov, M. Froehlicher, M. Froment and A. Hugot-le-Goff, J. Chimie Physique, 1975, 3, 275.

116 G. Blondeau, M. Froehlicher, M. Froment and A. Hugot-le-Goff, J. Microsc. Spectrosc. Electron., 1977, 2 , 27.

117 J. Yahalom and J. Zahavi, Electrochim. Acta, 1970, 15, 1429.

118 J.-L. Delplancke and R. Winand, Electrochim. Acta, 1988, 33, 1551.

119 G. K. Mor, O. K. Varghese, M. Paulose and C. A. Grimes, Sensor Lett., 2003, 1, 42.

120 C. Ruan, M. Paulose, O. K. Varghese and C. A. Grimes, Sol. Energy Mater. Sol. Cells, 2006, 90, 1283.

121 R. Beranek, H. Hildebrand and P. Schmuki, Electrochem. Solid-State Lett., 2003, 6, B12.

122 Q. Cai, M. Paulose, O. K. Varghese and C. A. Grimes, J. Mater. Res., 2005, 20, 230.

123 J. M. Macak, K. Sirotna and P. Schmuki, Electrochim. Acta, 2005, 50, 3679.

124 K. Shankar, G. K. Mor, H. E. Prakasam, S. Yoriya, M. Paulose,. O. K. Varghese and C. A. Grimes, Nanotech., 2007, 18, 065707.

125 M. Christophersen, J. Carstensen, K. Voigt and H. Föll, Phy. Stat. Sol. A, 2003, 197, 34.

126 K. Nakayama, T. Kubo, A. Tsubokura, Y. Nishikitani and H. Masuda, ECS Meeting Abstracts, 2006, 502, 819.

127 C. Richter, Z. Wu, E. Panaitescu, R. J. Willey and L. Menon, Adv. Mater., 2007, 19, 946.

128 C. Richter, E. Panaitescu, R.J. Willey and L. Menon, J. Mater. Res., 2007, 22, 1624.

129 G. K. Mor, O. K. Varghese, M. Paulose K. Shankar and C. A. Grimes, Sol. Energy Mater. Sol. Cells, 2006, 90, 2011.

130 L. V. Taveira, J. M. Macák, H. Tsuchiya, L. F. P. Dick and P. Schmuki, J. Electrochem. Soc., 2005, 152, B405.

131 J. Wang, H. P. Li and R. Stevens, J. Mater. Sci., 1992, 27, 5397.

132 J. Sundqvist, A. Harsta, J. Aarik, K. Kukli and A. Aidla, Thin Solid Films, 2003, 427, 147.

133 M. Gilo and N. Croitoru, Thin Solid Films, 1999, 350, 203. 
134 A. J. Waldorf, J. A. Dobrowolski, B. T. Sullivan and L. M. Plante, Appl. Opt., 1993, 32, 5583.

135 S. Capone, G. Leo, R. Rella, P. Siciliano, L. Vasanelli, M. Alvisi, L. Mirenghi and A. Rizzo, J. Vac. Sci. Technol. A, 1998, 16, 3564.

136 M. J. Esplandiu, L. B. Avalle and V. A. Macagno, Electrochim. Acta, 1995, 40, 2587.

137 M. J. Esplandiu, E. M. Patrito and V. A. Macagno, Electrochim. Acta, 1995, 40, 809.

138 M. Ohtaki, J. Peng, K. Eguchi and H. Arai, Sens. Actuat. B: Chem., 1993, 13-14, 495.

139 G. H. Hutching and S. H.Taylor, Catal. Today, 1999, 49, 105.

140 R. I. Aagard, Appl. Phys. Lett., 1975, 27, 605.

141 B. Ohtani, K. Iwai, S. Nishimoto and T. Inui, J. Electrochem. Soc., 1994, 141, 2439.

142 C. V. D'alkaine, L. M. M. De Souza and F. C. Nart, Corros. Sci., 1993, 34, 109.

143 L. Young, 'Anodic Oxide Films', Academic Press, London, 1961.

144 R. L. Karlinsey, Electrochem. Commun., 2005, 7, 1190.

145 J. Choi, J. H. Lim, J. Lee and K.J. Kim, Nanotech., 2007, 18, 055603.

146 J. L. Zhao, X. X. Wang, R. Q. Xu, Y. J. Mi and Y. X. Li, Electrochem. Solid-State Lett., 2007, 10, C31.

147 Y. Masuda, S. Wakamatsu and K. Koumoto, J. Eur. Ceram. Soc., 2004, 24, 301.

148 K. Kamada, M. Mukai and Y. Matsumoto, Electrochim. Acta, 2004, 49, 321.

149 A. Mozalev, M. Sakairi, I. Saeki and H. Takahashi, Electrochim. Acta, 2003, 48, 3155.

150 A. I. Vorobyova and E. A. Outkina, Thin Solid Films, 1998, 324, 1.

151 W. S. Kim, J. H. Kim, J. H. Kim, K. H. Hur and J. Y. Lee, Mater. Chem. Phys., 2003, 79, 204.

152 D. A.Vermilea, J. Electrochem. Soc., 1957, 104, 427.

153 I. V. Sieber and P. Schmuki, J. Electrochem. Soc., 2005, 152, C639.

154 H. Kawasaki, J. Namba, K. Iwatsuji, Y. Suda, K. Wada, K. Ebihara and T. Ohshima, Appl. Surf. Sci., 2002, 197-198, 547.

155 W. Gopel and D. Schierbaum, Sens. Actuat. B: chem., 1995, 26, 1.

156 S. Badilescu and P. V. Ashrit, Solid State Ionics, 2003, 158, 187.

157 E. Ozkan, S. H. Lee, P. Liu, C. E. Tracy, F. Z. Tepehan, J. R. Pitts and S. K. Deb, Solid State Ionics, 2002, 149, 139. 
158 A. E. Aliev and H. W. Shin. Solid State Ionics, 2002, 154-155, 425.

159 A. Antonaia, M. L. Addonizio, C. Minarini, T. Polichetti and M. V-Antisari, Electrochim. Acta, 2001, 46, 2221.

160 C. G. Granqvist, Electrochim. Acta, 1999, 44, 3005.

161 R. D. Rauh, Electrochim. Acta, 1999, 44, 3165.

162 S. H. Lee, H. M. Cheong, J. G. Zhang, A. Mascarenhas, D. K. Benson and S. K. Deb, Appl. Phys. Lett., 1999, 74, 242.

163 M. Sun, N. Xu, Y. W. Cao, J. N. Yao and E. G. Wang, J. Mater. Res., 2000, 15, 927.

164 A. I. Gavrilyuk, Electrochim. Acta, 1999, 44, 3027.

165 F. Di Quarto and A. Di Paola, Electrochim. Acta, 1981, 26, 1177.

166 D. J. Beckstead, G. M. Pepin and J. L. Ord, J. Electrochem. Soc., 1989, 136, 362.

167 J. L. Ord and D. J. De Smet, J. Electrochem. Soc., 1992, 139, 359.

168 H. Tsuchiya, J. M. Macak, I. Sieber, L. Taveira, A. Ghicov, K. Sirotna and P. Schmuki, Electrochem. Commun., 2005, 7, 295.

169 S. Berger, H. Tsuchiya, A .Ghicov and P. Schmuki, Appl. Phys. Lett., 2006, 88, 203119.

170 N. R. De Tacconi, C. R. Chenthamarakshan, G. Yogeeswaran, A. Watcharenwong, R. S. de

Zoysa, N. A. Basit and K. Rajeshwar, J. Phys. Chem. B, 2006, 110, 25347.

171 R. G. Keil and R. E. Solomon, J. Electrochem. Soc., 1968, 115, 628.

172 R. G. Keil and R. E. Solomon, J. Electrochem. Soc., 1965, 112, 643.

173 B. H. Ellis, M. A. Hopper and D. J.De Smet, J. Electrochem. Soc., 1971, 118, 860.

174 J. L. Ord, S. D. Bishop and D. J. DeSmet, J. Electrochem. Soc., 1991, 138, 208.

175 M. R. Arora and R. Kelly, J. Mater. Sci., 1977, 12, 1673.

176 M. R. Arora and R. Kelly, J. Electrochem. Soc., 1973, 120, 128.

177 W. D. Mackintosh and H. H. Plattner, J. Electrochem. Soc., 1976, 123, 523.

178 F. A. Chudnovskii and G. B. Stefanovich, J. Solid State Chem., 1992, 98, 137.

179 M. B. Lewis and R. A. Perkins, J. Electrochem. Soc., 1979, 126, 544.

180 S. Hornkjøl and I. M. Hornkjøl, Electrochim. Acta, 1991, 36, 577.

181 F. M. Al-Kharafi and W. A. Badawy, Electrochim. Acta, 1997, 42, 579.

182 J. P. Schreckenbach, K. Witke, D. Butte and G Marx, Fresenius J. Anal. Chem., 1999, 363, 211. 
183 T. Yamaguchi, Catal. Today, 1994, 20, 199.

184 K. Tanabe and T. Yamaguchi, Catal. Today, 1994, 20, 185.

185 B. Cox, J. Electrochem. Soc., 1970, 117, 654.

186 R. A. Ploc and M. A. Miller, J. Nucl. Mater., 1977, 64, 71.

187 H. Tsuchiya and P. Schmuki, Electrochem. Commun., 2004, 6, 1131.

188 R. C. Spooner, Nature, 1956, 178, 1113.

189 R. S. Hinde, E. A. Kellet and P. H. Harris, Nature, 1959, 183, 39.

190 R. K. Hart, Trans. Faraday. Soc., 1954, 50, 269.

191 M. S. Hunter, P. F. Towner and D. L. Robinson, Proc. Amer. Electroplaters Soc. 46th Ann. Conf., 1959, 3, 220.

192 W. J. Bernard, and J. J. Randall Jr, J. Electrochem. Soc., 1961, 108, 822.

193 G. D. Bengough and J. M. Stuart, Brit. Patent, 1925, 223995.

194 C. H. Giles, H. V. Mehta, C. E. Stewart and R. V. R. Subramanian, J. Chem. Soc., 1954, 4360.

195 B. M. Schenk, Werkstoff aluminium und seine anodische oxidation, Verlag A. Francke, Berne, 1948.

196 T. P. Hoar and G. C. Wood Electrochim. Acta, 1962, 7, 333.

197 M. Kokonou, C. Rebholz, K. P. Giannakopoulos and C. C. Doumanidis, Microelectr. Eng., 2008, 85, 1186.

198 T. Asada, Jpn. Patent, 1969, 824505.

199 S. Kawai, and I. Ishiguro, J. Electrochem. Soc., 1975, 122, 32.

200 S. Kawai and I. Ishiguro, J. Electrochem. Soc., 1976, 123, 1047.

201 G. Sauer, G. Brehm, S. Schneider, K. Nielsch, R. B. Wehrspohn, J. Choi, H. Hofmeister and U. Gosele, J. Appl. Phys., 2002, 91, 3243.

202 S. Shingubara, O. Okino, Y. Sayama, H. Sakaue and T. Takahagi, Jpn. J. Appl. Phys., 1997, $36,7791$.

203 J. Sha, J. J. Niu, X. Y. Ma, J. Xu, X. B. Zhang, Q. Yang and D. R. Yang, Adv. Mater., 2002, $14,1219$.

204 T. Yanagishita, K. Nishio and H. Masuda, Adv. Mater., 2005, 17, 2241. 
205 J. Qin, J. Nogués, M. Mikhaylova, A. Roig, J. S. Muňoz and M. Muhammed, Chem. Mater., 2005, 17, 1829.

206 A. Ghicov and P. Schmuki, Chem. Commun., 2009, 2791.

207 C. C. Wang, Z. Zhang and J. Y. Ying, Nanostruct. Mater., 1997, 9, 583.

208 Z. Zhang, C. C. Wang, R. Zakaria and J. Y. Ying, J. Phys. Chem. B, 1998, 102, 10871.

209 J. M. Macak, M. Zlamal, J. Krysa and P. Schmuki, Small, 2007, 3, 300.

210 S. U. M. Khan, M. Al-Shahry and W. B. Ingler, Science, 2002, 297, 2243.

211 M. J. Madou and S. R. Morrison, Chemical Sensing with Solid State Devices, Academic Press, New York, 1989.

212 C. C. Wang, S. A. Akbar and M. J. Madou, J. Electroceramics, 1998, 2, 273.

213 J. Desilvestro, M. Grä tzel, L. Kavan, J. Moser and J. Augustynski, J. Am. Chem. Soc., 1985, 107, 2988.

214 M. R. Hoffmann, S. T. Martin, W. Choi and D. W. Bahnemann, Chem. Rev., 1995, 95, 69.

215 S. P. Albu, A. Ghicov, J. M. Macak, R. Hahn and P. Schmuki, Nano Lett., 2007, 7, 1286.

216 H. Tsuchiya, J. M. Macak, L. Muller, J. Kunze, F. Muller, P. Greil, S. Virtanen and P.

Schmuki, J. Biomed. Mater. Res. Part A, 2006, 77A, 534.

217 O. K. Varghese, X. P. Yang, J. Kendig, M. Paulose, K. Zeng, C. Palmer, K. G. Ong and C.

A. Grimes, Sensor Lett., 2006, 4, 120.

218 J. F. Qi, J. M. White, A. M. Belcher and Y. Masumoto, Chem. Phys. Lett., 2003, 372, 763.

219 V. F. Henly, 'Anodic oxidation of aluminium and its alloys', Pergamon Press, Oxford, 1982.

220 J. Fu, S. Cherevko and C. H. Chung, Electrochem. Commun., 2008, 10, 514.

221 H. Masuda, F. Hasegwa and S. Ono, J. Electrochem. Soc., 1997, 144, L127.

222 I. De Graeve, H. Terryn and G. E. Thompson, J. Electrochem. Soc., 2003, 150, B158.

223 M. A. Kashi and A. Ramazani, J. Phys. D: Appl. Phys., 2005, 38, 2396.

224 D. Lo and R. A. Budiman, J. Electrochem. Soc., 2007, 154, C60.

225 Z. X. Su, G. Hahner and W. Z. Zhou, J. Mater. Chem., 2008, 18, 5787.

226 A. Güntherschulze and H. Betz, Z. Phys., 1934, 92, 367.

227 N. Cabrera and N. F. Mott, Rep. Prog. Phys., 1948, 12, 163.

228 K. R. Hebert, H. Q. Wu, T. Gessmann and K. G. Lynn, J. Electrochem. Soc., 2001, 148, B92. 
229 R. C. Huang, K. R. Hebert, T. Gessmann and K. G. Lynn, J. Electrochem. Soc., 2004, 151, B227.

230 C. Y. Chao, L. F. Lin and D. D. Macdonald, J. Electrochem. Soc., 1981, 128, 1187.

231 L. F. Lin, C. Y. Chao and D. D. Macdonald, J. Electrochem. Soc., 1981, 128, 1194.

232 T. Martin and K. R. Hebert, J. Electrochem. Soc., 2001, 148, B101.

233 B. J. Wiersma and K. R. Hebert, J. Electrochem. Soc., 1991, 138, 48.

234 Z. Wu, C. Richter and L. Menon, J. Electrochem. Soc., 2007, 154, E8.

235 M. S. Hunter and P. Fowle, J. Electrochem. Soc., 1954, 101, 481.

236 G. C. Wood and J. P. O’Sullivan, Electrochim. Acta, 1970, 15, 1865.

237 S. Ono and N. Masuko, Surf. Coat. Tech., 2003, 169-170, 139.

238 C. J. Dell'Oca and P. J. Fleming, J. Eletrochem. Soc., 1976, 123, 1487.

239 S. Ono and K. Takeda, Proceedings of the Eighth International Symposium on Passivity of Metals and Semi-conductors, 2000, 931.

240 R. Asahi, T. Morikawa, T. Ohwaki, K. Aoki and Y. Taga, Science, 2001, 293, 269.

241 P. A. Connor, K. D. Dobson and A. J. McQuillan, Langmuir, 1999, 15, 2402.

242 L. V. Taveira, J. M. Macak, H. Tsuchiya, L. F. P. Dick and P. Schmuki, J. Electrochem. Soc., 2005, 152, B405.

243 G. K. Mor, O. K. Varghese, M. Paulose, N. Mukherjee and C. A. Grimes, J. Mater. Res., 2003, 18, 2588.

244 J. Choi, R. B. Wehrspohn, J. Lee and U. Gosele, Electrochim Acta, 2004, 49, 2645.

245 Z. D. Zivkovic and B. Dobovisek, J. Thermal Anal. Cal., 1977, 12, 207.

246 X. F. Wang and L. Andrews, J. Phys. Chem. A, 2005, 109, 10689.

247 J. F. Banfield, D. R. Veblen and D. J. Smith, Am. Mineral., 1991, 76, 343.

248 T. P. Feist and P. K. Davies, J. Solid State Chem., 1992, 101, 275.

249 Z. X. Su and W. Z. Zhou, J. Mater. Chem., 2009, 19, 2301.

250 N. G. Park, J. van de Lagemaat and A. J. Frank, J. Phys. Chem. B, 2000, 104, 8989.

251 Z. Ma, Y. H. Yue, X. Y. Deng and Z. Gao, J. Mol. Catal. A: Chem., 2002, 178, 97.

252 A. Wold, Chem. Mater., 1993, 5, 280.

253 S. Yoriya, G. K. Mor, S. Sharma and C. A. Grimes, J. Mater. Chem., 2008, 18, 3332.

254 F. Tielens, L. Gracia, V.Polo and J. Andres, J. Phys. Chem. A, 2007, 111, 13255. 
255 D. Rai, H. Joshi, A. D. Kulkarni, S. P. Gejji and R. K.Pathak, J. Phys. Chem. A, 2007, 111, 9111.

256 I. Rozas, I. Alkorta and J. Elguero, Chem. Phys. Lett., 1997, 275, 423.

257 I. Mata, E. Molins, E. Alkorta and E. Espinosa, J. Chem.Phys., 2009, 130, 044104.

258 M. Ramos, I. Alkorta, J. Elguero, N. S. Golubev, G. S. Denisov, H. Benedict and H.

H.Limbach, J. Phys. Chem. A, 1997, 101, 9791.

259 S. Shaik, S. P. de Visser and D. Kumar, J. Am. Chem. Soc., 2004, 126, 11746.

260 E. Marcos, J. M. Anglada and R. Crehuet, Phys. Chem. Chem. Phys., 2008, 10, 2442.

261 J.-S. McEwen, P. Gaspard, F. Mittendorfer, T. V. de Bocarme and N. Kruse, Chem. Phys. Lett., 2008, 452, 133.

262 S. J. Suresh and A. V. Satish, J. Chem. Phys., 2006, 124, 074506.

263 C Y.-K. hoe, E. Tsuchida and T. Ikeshoji, J. Chem. Phys., 2007, 126, 154510.

264 A. D. Becke, J. Chem. Phys., 1993, 98, 5648.

265 C. Lee, W. Yang and R. G. Parr, Phys. ReV. B, 1988, 37, 785.

266 R. Krishnan, J. S. Binkley, R. Seeger and J. A. Pople, J. Chem. Phys., 1980, 72, 650.

267 T. Clark, J. Chandrasekhar, G. W. Spitznagel and P. V. R. Schleyer, J. Comput. Chem., 1983, 4, 294.

268 This and similar levels are well suited to study properties of water such as dipole moment, binding energy of the dimer, etc., see, e.g.: (a) W. Koch, M. Holthausen, C. A Chemist's Guide to DensityFunctional Theory, 2nd. Ed, Wiley-VCH: Weinheim, 2001. Also, the experimental proton affinity of water is reproduced within $0.5 \mathrm{kcal}$ mol-1 at the B3LYP/6-311+G(d,p) level: (b) G. A. DiLabio, D. A. Pratt, A. D. LoFaro, and J. S. Wright, J. Phys. Chem. A, 1999, 103, 1653.

269 S. Miertus, E. Scrocco and J. Tomasi, Chem. Phys., 1981, 55, 117.

270 B. Mennucci and J. Tomasi, J. Chem. Phys., 1997, 106, 5151.

271 V. Barone, M. Cossi and J. Tomasi, J. Chem. Phys., 1997, 107, 3210.

272 M. J. Frisch, G. W. Trucks, H. B. Schlegel, G. E. Scuseria, M. A. Robb, J. R. Cheeseman, J. A. Montgomery Jr, T. Vreven, K. N. Kudin, J. C. Burant, J. M. Millam, S. S. Iyengar, J. Tomasi, V. Barone, B. Mennucci, M. Cossi, G. Scalmani, N. Rega, G. A. Petersson, H. Nakatsuji, M. Hada, M. Ehara, K. Toyota, R. Fukuda, J. Hasegawa, M. Ishida, T. Nakajima, 
Y. Honda, O. Kitao, H. Nakai, M. Klene, X. Li, J. E. Knox, H. P. Hratchian, J. B. Cross, C. Adamo, J. Jaramillo, R. Gomperts, R. E. Stratmann, O. Yazyev, A. J. Austin, R. Cammi, C. Pomelli, J. W. Ochterski, P. Y. Ayala, K. Morokuma, G. A. Voth, P. Salvador, J. J. Dannenberg, V. G. Zakrzewski, S. Dapprich, A. D. Daniels, M. C. Strain, O. Farkas, D. K. Malick, A. D. Rabuck, K. Raghavachari, J. B. Foresman, J. V. Ortiz, Q. Cui, A. G. Baboul, S. Clifford, J. Cioslowski, B. B. Stefanov, G. Liu, A. Liashenko, P. Piskorz, I. Komaromi, R. L. Martin, D. J. Fox, T. Keith, M. A. Al-Laham, C. Y. Peng, A. Nanayakkara, M. Challacombe, P. M. W. Gill, B. Johnson, W. Chen, M. W. Wong and C. Gonzalez, Gaussian 03, revision E01; Gaussian, Inc.: Pittsburgh, PA, 2003.

273 B. L. Trout and M. Parrinello, Chem. Phys. Lett., 1998, 288, 343.

274 M. Sprik, Chem. Phys., 2000, 258, 139.

275 The precise choice of this distance is not overly critical, as $\Delta \mathrm{E}$ values differ typically by less than $10 \%$ when evaluated at, e.g., r1 ) $1.8 \AA$ instead of $2.0 \AA$.

276 Using $\cos 2 \mathrm{R}$ instead of a simple cosine as trial function affords a worse fit (not shown).

$2771 \mathrm{kcal} \mathrm{nm} \mathrm{mol-1} \mathrm{V-1} \mathrm{)} 4.18 \times 10-6 \mathrm{C}$ m mol-1. Error bars from linear regressions are all smaller than $(0.01 \mathrm{kcal} \mathrm{nm} \mathrm{mol-1} \mathrm{V-1.}$

278 Exactly the same value is obtained with $6-311++\mathrm{G}(\mathrm{d}, \mathrm{p})$ basis, i.e., including diffuse functions on $\mathrm{H}$ atoms.

279 V. A. Ranea, W. F. Schneider and I. Carmichael, Surf. Sci., 2008, 602,268.

280 J. M. Wittbrodt, W. L. Hase and H. B. Schlegel, J. Phys. Chem. B, 1998, 102, 6539.

281 O. Teschke, G. Ceotto and E. F. de Souza, Chem. Phys. Lett., 2000, 326, 328.

282 O. Teschke, G. Ceotto and E. F. de Souza, Phys. Rev. E, 2001, 64, 011605.

283 D. A. Cherepanov, B. A. Feniouk, W. Junge and A. Y. Mulkidjanian, Biophys. J., 2003, 85, 1307.

284 Since the computations involved both an external field and a dielectric continuum, they have implicitly used the corresponding local field.

285 L. Rayleigh, Philos. Mag., 1916, 32, 529.

286 A. R. Rao and P. C. Biswal, Acta Mech., 2001, 151, 61.

287 V. Fleury, J. N. Chazalviel and M. Rosso, Phys. Rev. Lett., 1992, 68, 492.

288 Y. L. Han and D. G. Grier, J. Chem. Phys., 2005, 122, 164701. 
289 G. Marshall, E. Mocskos, F. V. Molina and S. Dengra, Phys. Rev. E, 2003, 68, 021607.

290 M. Orlik, J. Phys. Chem. B, 1999, 103, 6629.

291 M. Orlik, K. Doblhofer and G. Ertl, J. Phys. Chem. B, 1998, 102, 6367.

292 Y. L. Han and D. G. Grier, J. Chem. Phys., 2006, 125, 144707.

293 V. Fleury, J. N. Chazalviel and M. Rosso, Phys. Rev. E, 1993, 48, 1279.

294 T. Pundik, I. Rubinstein and B. Zaltzman, Phys. Rev. E, 2005, 72, 061502.

295 D. C. Rapaport, Phys. Rev. E, 2006, 73, 025301.

296 P. A. Vazquez, G. E. Georghiou and A. Castellanos, J. Phys. D: Appl. Phys., 2008, 41, 175303.

297 F. Zhang, X. H. Liu, C. F. Pan, and J. Zhu, Nanotech., 2007, 18, 345302.

298 M. Golubitsky, J. W. Swift and E. Knobloch, Physica D, 1984, 10, 249.

299 E. Bodenschatz, W. Pesch and G. Ahlers, Annu. Rev. Fluid Mech., 2000, 32, 709.

300 J. N. Chazalviel, Phys. Rev. A, 1990, 42, 7355. 


\section{Publications}

1 Z. X. Su and W. Z. Zhou, 'Formation mechanism of porous anodic aluminium and titanium oxides', Adv. Mater., 2008, 20, 3663.

2 Z. X. Su, G. Hahner and W. Z. Zhou, 'Investigation of the pore formation in anodic aluminium oxide', J. Mater. Chem., 2008, 18, 5787.

3 Z. X. Su and W. Z. Zhou, 'Porous anodic metal oxides', Science Foundation in China, 2008, 16,36 .

4 Z. X. Su and W. Z. Zhou, 'Formation, microstructures and crystallization of anodic titanium oxide tubular arrays', J. Mater. Chem., 2009, 19, 2301.

5 Z. X. Su, M. Bühl and W. Z. Zhou, 'Dissociation of water during formation of anodic aluminium oxide', J. Am. Chem. Soc., 2009, 131, 8697.

6 S. J. Lv, Z. X. Su, J. Sha and W. Z. Zhou, 'Ionic nanoconvection in anodization of aluminium plate', Chem. Comm., 2009, 5639.

7 W. B. Yue, C. Randorn, P. S. Attidekou, Z. X. Su, J. T. S. Irvine and W. Z. Zhou, Syntheses, Li Insertion, and photoactivity of mesoporous crystalline $\mathrm{TiO}_{2}$ ', Adv. Funct. Mater., 2009, 19, 2826. 